\title{
Abhängige Sätze in einem fragebasierten Diskursmodell
}

\author{
Dissertation \\ zur Erlangung des philosophischen Doktorgrades \\ an der Philosophischen Fakultät der \\ Georg-August-Universität Göttingen
}

\author{
vorgelegt von \\ Mailin Ines Antomo \\ aus Wiesbaden
}

Göttingen 2012 
1. Gutachter: Prof. Dr. Markus Steinbach

2. Gutachterin: Prof. Dr. Anke Holler

Tag der mündlichen Prüfung: 08.08.2013 


\section{Danksagung}

An dieser Stelle möchte ich jenen danken, die zum Gelingen dieser Arbeit durch ihre Unterstützung beigetragen haben.

Zuallererst möchte ich mich bei Markus Steinbach für die engagierte Betreuung und wissenschaftliche Begleitung während der letzten Jahre bedanken. Mit zahlreichen konstruktiven Ratschlägen, aber auch seiner Neugierde und Begeisterung leistete er für die Entwicklung dieser Arbeit einen entscheidenden Beitrag. Ein großes Dankeschön gilt außerdem Anke Holler, die mich während der Anfertigung meiner Dissertation begleitet und mit wichtigen Anregungen unterstützt hat.

Bedanken möchte ich mich auch bei Edgar Onea, Thomas Weskott und Yingyang Xue für ihre kompetente Beratung bei der Erstellung und Auswertung des Fragebogens.

Für viele hilfreiche Anregungen und vor allem ein hervorragendes Arbeitsklima möchte ich mich bei meinen Göttinger KollegInnen Petra-Kristin Bonitz, Miriam Ellert, Annika Herrmann, Jana Hosemann, Annika Hübl, Joost Kremers, Anja Müller, Nina-Kristin Pendzich, Karin Peschke, Angelika Schmidt, Augustin Speyer und Jeanine Wein ganz herzlich bedanken. Ein aufrichtiges Dankeschön gebührt weiterhin Karen Laubinger-Jorks, Yuka Richter und Romina Silvestre für die gute Zusammenarbeit.

Ohne den produktiven Ideenaustausch mit meinen damaligen KollegInnen am DFGGraduiertenkolleg „Satzarten: Variation und Interpretation“ in Frankfurt a. M. wäre diese Arbeit nicht in dieser Form zustande gekommen. Besonders danken möchte ich Elena Castroviejo-Miró und Shin-Sook Kim. Zentrale Impulse verdanke ich außerdem Katrin Axel-Tober, Liliane Haegeman, Richard Larson und Jörg Meibauer.

Mein besonderer Dank gilt meinen Eltern, meiner Schwester, meiner Großmutter und meinem Mann für ihr Vertrauen in mich und ihre fortwährende Unterstützung. 


\section{Abstract}

It has been argued that each discourse aims to answer an explicit or implicit question, the Question Under Discussion (QUD) (see Klein/von Stutterheim 1992 and Simons et al. 2011). The QUD affects not only the interpretation of utterances, but it also affects the linguistic form of what is said. By several means, the grammar of a language marks content that aims to answer the actual QUD as at-issue content, whereas additional side-comments are marked as not-at-issue. In this way, grammar gives some hint about what kind of QUD an utterance may answer.

Bartsch (1978), Hartmann (1984), Posner (1972), and von Stutterheim (1989, 168) assume that sentential subordination conventionally marks not-at-issueness. However, already Brandt (1989, 1996), Holler (2009), and Simons (2007) observe that embedded clauses can express at-issue content as shown in the following example from Simons (2007, 1035):

Q: Where was Harriet yesterday?

B: Henry discovered that she had a job interview at Princeton.

Here, what is clearly relevant to the QUD is the content of the embedded clause. Thus, whereas the clause embedded by the semifactive verb discover can express at-issue content, the same is not possible for the complement of a factive predicate:

Q: Where was Harriet yesterday?

B: \#Henry ignores that she had a job interview at Princeton.

In this dissertation, I will investigate different types of dependent clauses, showing that they form two groups with respect to their discourse function. One group of dependent clauses is conventionally marked as not-at-issue and therefore cannot express an information that is relevant to the current QUD. Characteristic features of these dependent clauses are that they project under the scope of an entailmentcancelling operator and that they cannot be denied directly, what is going to be confirmed by the results of a questionary. In contrast, depending on the context, dependent clauses of the second group can express at-issue or not-at-issue content. Therefore, only a subset of the totality of dependent clauses can be used to answer the QUD.

Interestingly, only dependent clauses that express at-issue content can undergo V2 movement in German, which is subsumed under the notion of Main Clause Phenomena. The licensing of V2 in German dependent clauses is typically explained in terms of presupposition and assertion. It is assumed that only asserted clauses can undergo V2 movement. In this dissertation, however, I will show that the Assertion 
Hypothesis leads to wrong predictions. Instead of relying on assertional force, I will argue that V2 in German dependent clauses is an optional marker of at-issueness. The contrast between dependent clauses that can express at-issue content and those that cannot is going to be investigated in more detail by comparing temporal and causal clauses, whereas only the latter can be used to answer the current QUD. Based on the results of Johnston (1994), I will show that this contrast can be traced back to a fundamental difference between the semantic contribution of temporal and causal conjunctions: Whereas causal weil expresses a relation between two events, temporal conjunctions such as bevor or nachdem are non-relational. As one-place predicates they map their IP-complement to a time interval i which restricts the nuclear scope of an overt or covert quantifier. As quantifier restrictions, temporal clauses are subject to an existence presupposition and therefore cannot be used to express at-issue content. 


\section{Inhaltsverzeichnis}

$\begin{array}{lll}\text { Abstract } & \text { v }\end{array}$

Abbildungsverzeichnis $\quad$ xi

Tabellenverzeichnis xiii

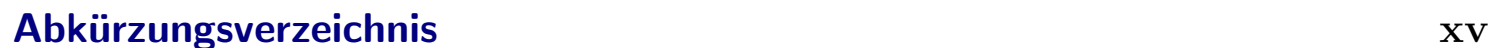

1 Einleitung 1

I Diskurssemantische und -pragmatische Aspekte von abhängigen Sätzen 5

2 Zur Interpretation von Sätzen im Kontext $\quad 7$

2.1 Der Kontext als Frage . . . . . . . . . . . . . . . . 7

2.1.1 Grundlagen .................... . . 7

2.1.2 Haupt- und Nebenstruktur . . . . . . . . . . . . . . 15

2.1.3 Sprachliche Markierung. . . . . . . . . . . . . . . . . . 19

2.1.4 Zusammenfassung . . . . . . . . . . . . . . . 23

2.2 Abhängige Sätze und die Beantwortung von Q . . . . . . . . . . . . 24

2.2.1 Grundlagen . . . . . . . . . . . . . . . . . . 24

2.2.2 Komplementsätze von Verben . . . . . . . . . . . . 27

2.2 .3 Adverbialsätze . . . . . . . . . . . . . . . . 33

2.2 .4 Relativsätze . . . . . . . . . . . . . . . . . . 38

2.2.5 Zusammenfassung . . . . . . . . . . . . . . . . 42

2.3 Zusammenfassung des Kapitels . . . . . . . . . . . . . . . . . . . 44

3 Typische Eigenschaften der Nebenstruktur $\quad 45$

3.1 Projektion . . . . . . . . . . . . . . . . . . 47

3.1.1 Grundlagen . . . . . . . . . . . . . . . 47

3.1 .2 Projektionsverhalten von abhängigen Sätzen . . . . . . . . 51

3.2 Widerspruchsstrategien . . . . . . . . . . . . . . 58

3.2 .1 Grundlagen . . . . . . . . . . . . . . . 58

3.2 .2 Bisherige Untersuchungen . . . . . . . . . . . . . . 61

3.2.2.1 Potts (2008) . . . . . . . . . . . . 61 
3.2.2.2 Amaral et al. (2011) . . . . . . . . . . . . 62

3.2.2.3 Xue/Onea (2011) . . . . . . . . . . . . . . . 64

3.2.2.4 Zusammenfassung ............... . . . . . 67

3.2.3 Fragebogenstudie . . . . . . . . . . . . . . . . 68

3.2.3.1 Aufbau und Durchführung . . . . . . . . . . 68

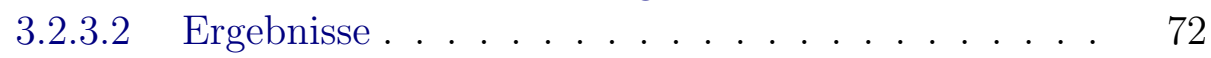

3.2.3.3 Diskussion . . . . . . . . . . . . . 75

3.3 Zusammenfassung des Kapitels . . . . . . . . . . . . . . . . . . . 79

4 Präsupponiertheit und At-issueness $\quad \mathbf{8 1}$

4.1 Distinktive Merkmale von Präsuppositionen . . . . . . . . . . . . . 81

4.1.1 Präsuppositionen müssen wahr sein . . . . . . . . . . . . 81

4.1.2 Präsuppositionen müssen im Common Ground gegeben sein 83

4.1.3 Präsuppositionen sind anaphorisch . . . . . . . . . . 88

4.1.3.1 Hintergrund: Dynamische Semantik . . . . . . . . . 89

4.1.3.2 Die Bindungstheorie von Präsuppositionen . . . . . 92

4.1.3.3 Präsuppositionen in SDRT . . . . . . . . . . . . 95

4.1 .4 Zusammenfassung . . . . . . . . . . . . . . . . 101

4.2 Welche Nebensätze sind präsupponiert? . . . . . . . . . . . . . . . . 101

4.3 Zusammenfassung des Kapitels . . . . . . . . . . . . . . . . 107

\section{Abhängige V2-Strukturen 109}

5 Ein erster Überblick: Wurzelphänomene in abhängigen Sätzen 111

$\begin{array}{llr}6 & \text { Lizenzierungskontexte } & 117\end{array}$

6.1 Komplementsätze . . . . . . . . . . . . . . . . . . . 117

6.2 Adverbialsätze . . . . . . . . . . . . . . . . . . . . 118

6.3 Relativsätze . . . . . . . . . . . . . . . . . . . . . . . . . . . . . . . . . . . . . 121

6.4 Zusammenfassung des Kapitels . . . . . . . . . . . . . . . . . . . . 124

7 Lizenzierungsbedingungen $\quad 127$

7.1 Bisherige Ansätze . . . . . . . . . . . . . . . . . . . . . . 128

7.1.1 Die Assertionshypothese . . . . . . . . . . . . . . 128

7.1.2 V2-Nebensätze und die Assertionshypothese . . . . . . . . . 131

7.2 V2-Stellung als grammatische Markierung für At-issueness . . . . . 142

7.3 Weitere Anwendungsbereiche der At-issueness-Hypothese . . . . . . 151

7.3.1 Andere abhängige V2-Strukturen . . . . . . . . . . . . . 151

7.3.2 Eingebettete Topikalisierung im Englischen . . . . . . . . . . 156

7.3.3 Unintegrierte Verbletztsätze . . . . . . . . . . . . . . . . 160

7.4 Zusammenfassung des Kapitels . . . . . . . . . . . . . . . . . . . . 164 
III Temporale und kausale Adverbialsätze im Vergleich $\quad 167$

8 Relationale und nicht-relationale Adverbiale $\quad 169$

8.1 Interaktion mit Negation . . . . . . . . . . . . . . . . . . . . . 170

8.2 Interaktion mit Quantoren . . . . . . . . . . . . . . . . . 175

8.3 Verhalten in Kopulakonstruktionen . . . . . . . . . . . . . . . . . . 181

8.4 Zusammenfassung des Kapitels . . . . . . . . . . . . . . . . . . . . 182

9 Semantische Eigenschaften von Temporal- und weil-Sätzen $\quad 183$

9.1 Temporalsätze . . . . . . . . . . . . . . . . . . 183

9.1.1 Denotation eines Intervalls . . . . . . . . . . . . . 183

9.1.2 Verortung des Hauptsatzes innerhalb des Intervalls . . . . . 196

9.1.3 Zur Präsupposition von Temporalsätzen . . . . . . . . . . . 202

9.2 Weil-Sätze . . . . . . . . . . . . . . . . . . 210

9.3 Zusammenfassung des Kapitels . . . . . . . . . . . . . . . . . 215

$\begin{array}{ll}10 \text { Schlussbemerkung } & 217\end{array}$

$\begin{array}{ll}\text { A Testsätze } & 223\end{array}$

$\begin{array}{ll}\text { Literaturverzeichnis } & 231\end{array}$ 
Inhaltsverzeichnis 


\section{Abbildungsverzeichnis}

$2.1 \quad$ Exemplarischer Diskursbaum . . . . . . . . . . . . . . 11

$3.1 \quad$ Ergebnisse des Fragebogens nach Gruppen . . . . . . . . . . . . . 73

3.2 Häufigkeitsverteilung nach Gruppen . . . . . . . . . . . . . . . . 74

3.3 Ergebnisse des Fragebogens nach Konditionen . . . . . . . . . . . 75

3.4 Häufigkeitsverteilung Gruppe $2 \ldots \ldots$. . . . . . . . 76

3.5 Häufigkeitsverteilung Gruppe $1 \ldots \ldots 77$

4.1 Präsuppositionen und [-at-issue]-Inhalte . . . . . . . . . . . . 102

7.1 Lizenzierungskontexte für Wurzelphänomene im Deutschen und im Englischen . . . . . . . . . . . . . . . . . . . 160

$9.1 \quad$ Verortung einer Ereignisbeschreibung auf einem Zeitstrahl . . . . 184

9.2 Die drei Intervalle der temporalen Nachbarschaft . . . . . . . . . 185

9.3 Denotation eines während-Satzes . . . . . . . . . . . . . . . . 187

$9.4 \quad$ Denotation eines bevor-Satzes . . . . . . . . . . . . . . . 188

9.5 Denotation eines nachdem-Satzes . . . . . . . . . . . . . . . 189

9.6 Restriktion eines Allquantors durch einen während-Satz . . . . . 190

9.7 Restriktion eines (koverten) Existenzquantors durch einen währendSatz . . . . . . . . . . . . . . . . . . 191

9.8 Restriktion eines (koverten) Existenzquantors durch einen bevorSatz . . . . . . . . . . . . . . . . . . 194

9.9 Gegenüberstellung der ein- und zweistelligen Analyse von temporalen Subjunktionen . . . . . . . . . . . . . . . . . 195

9.10 Positionierung des Hauptsatzes innerhalb von i . . . . . . . . . . . 196

9.11 Temporale Skala und Informativität . . . . . . . . . . . . . . . . 197

9.12 Semantischer Unterschied zwischen nachdem und sowie . . . . . 201

10.1 Präsuppositionen und At-issueness . . . . . . . . . . . . . . 219 
Abbildungsverzeichnis 


\section{Tabellenverzeichnis}

2.1 Nebensätze und die Beantwortung von Q . . . . . . . . . . 42

$3.1 \quad$ Projektionsverhalten von Nebensätzen . . . . . . . . . . 58

3.2 Ergebnisse, Experiment 1 aus Xue/Onea (2011) . . . . . . . . . 65

3.3 Ergebnisse, Experiment 2 aus Xue/Onea (2011) . . . . . . . . . 66

3.4 Ergebnisse des Fragebogens nach Konditionen, Gruppe 2 . . . . . 74

3.5 Ergebnisse des Fragebogens nach Konditionen, Gruppe 1 . . . . . 75

3.6 Typische Eigenschaften der Haupt- und Nebenstruktur . . . . . . 79

4.1 Klassifikation der Nebensätze nach At-issueness und Präsupponiertheit . . . . . . . . . . . . . . . . . 108

6.1 Lizenzierungskontexte für V2-Stellung . . . . . . . . . . . 125

7.1 At-issueness und abhängige V2-Stellung . . . . . . . . . . . . 143

7.2 Merkmalsverteilung bei Nebensätzen . . . . . . . . . . . . . . . . 143

7.3 Lizenzierungskontexte im Vergleich: Deutsch und Englisch . . . . 158

10.1 Die diskutierten strukturellen und funktionalen Eigenschaften im Überblick . . . . . . . . . . . . . . . . . . . . . . . 222 
Tabellenverzeichnis

xiv 


\section{Abkürzungsverzeichnis}

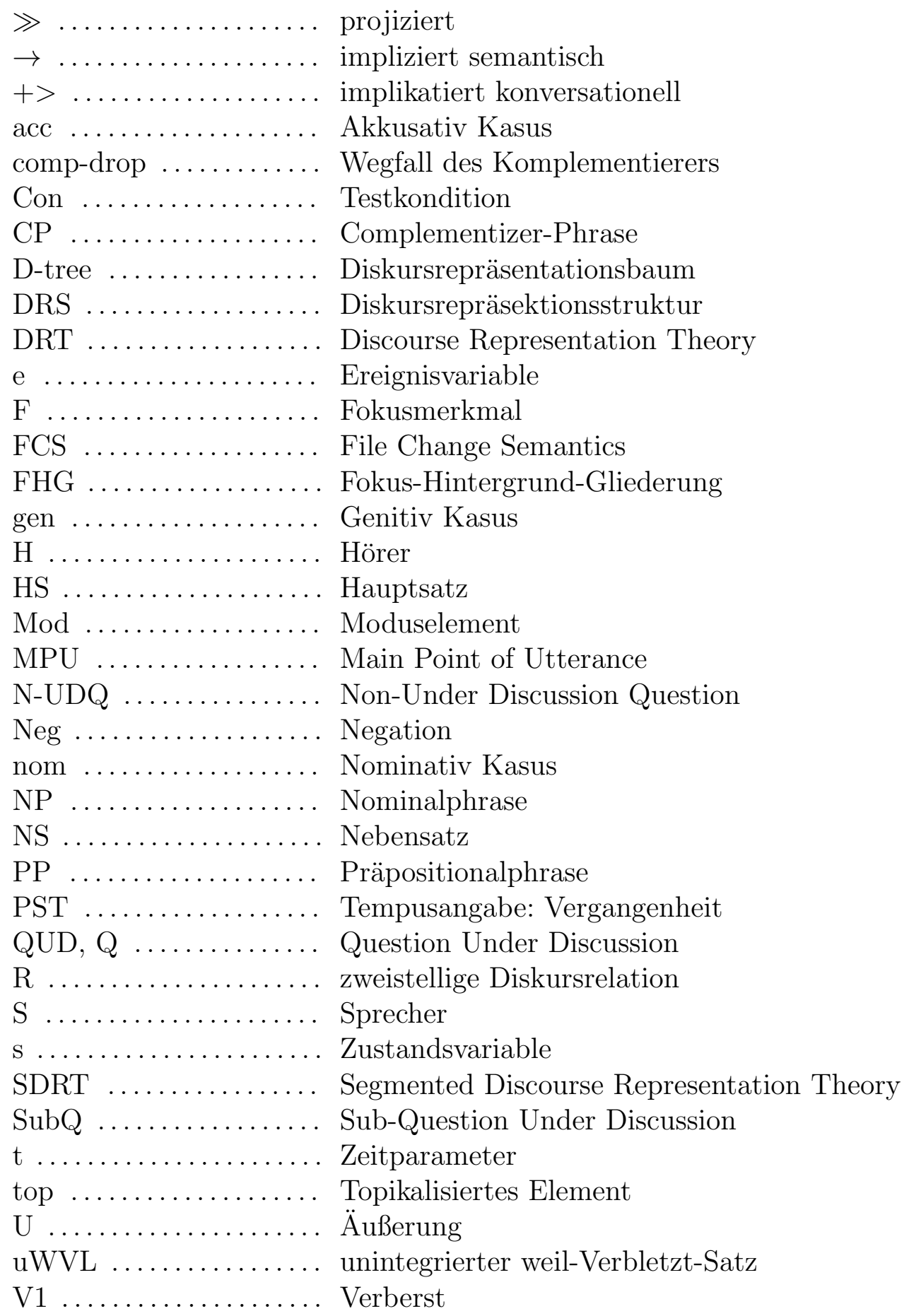


Abkürzungsverzeichnis

$\mathrm{V} 2 \ldots \ldots \ldots \ldots \ldots$ Verbzweit

VL ............... Verbletzt 


\section{Einleitung}

Die vorliegende Arbeit zielt auf eine Beschreibung der textstrukturierenden Funktion von abhängigen Sätzen in einem fragebasierten Diskursmodell ab. ${ }^{1}$ In neueren diskurssemantischen Ansätzen wird der Kontext häufig als Frage modelliert, die Quaestio in der Terminologie von Klein/von Stutterheim (1992), von Stutterheim (1989) und von Stutterheim/Klein (1989) bzw. die Question Under Discussion (kurz: QUD) in dem fragebasierten Diskursmodell nach Beaver/Clark (2008), Büring (2003), Roberts (1996), Roberts et al. (2009) und Simons et al. (2011). Dabei wird davon ausgegangen, dass jedem Diskurs eine explizite oder implizite Frage zugrunde liegt, auf deren Beantwortung der gesamte Diskurs abzielt. Typischerweise dient dabei der Inhalt von Hauptsätzen der Klärung der aktuellen QUD, wohingegen Nebensätze häufig zusätzliche Hintergrundinformationen liefern wie in dem folgenden Beispiel:

A: Wo ist denn Maria?

B: Sie ist nach Hause gegangen, weil sie müde war.

Die Annahme, dass Subordination ein Mittel zur kommunikativen Gewichtung ist, findet bereits in der alten Schulweisheit „Hauptsachen in Hauptsätze, (...) Nebensachen in Nebensätze" (hier nach der Stilfibel von Reiners 1951, 79) ihren Niederschlag, welche auch in ähnlicher Form in der germanistischen Forschung zur Reliefgebung vertreten wird (vgl. beispielsweise Bartsch 1978, Hartmann 1984 und Posner 1972 oder auch von Stutterheim 1989, 168). Bereits Brandt (1989, 1996), Holler (2009) und Simons (2007) beobachten jedoch, dass die kommunikative Relevanz des Gesagten anhand der Distinktion von Haupt- und Nebensätzen nicht eindeutig markiert wird. So können einige spezifische Nebensatztypen durchaus dazu verwendet werden, die kontextuell relevante Hauptinformation auszudrücken. Dies zeigt sich in dem folgenden Beispiel, in dem die QUD durch den Nebensatz beantwortet wird.

A: Wo ist denn Maria?

B: Peter glaubt, dass sie zum Friseur gegangen ist.

Allerdings sind nicht alle Nebensatztypen dazu geeignet, quaestiobezogene Informationen auszudrücken. Beispielsweise führt die Beantwortung einer Frage durch einen Komplementsatz des Verbs sich freuen zu einer nicht-wohlgeformten Äußerung:

${ }^{1}$ Abhängige Sätze (= Nebensätze) können integriert oder unintegriert auftreten. In Teil I werden vor allem kanonische, integrierte Nebensätze diskutiert, während in Teil II mit abhängigen V2-Strukturen auch unintegrierte Nebensätze in die Untersuchung miteinbezogen werden. 
A: Wo ist denn Maria?

B: \#Peter freut sich, dass sie zum Friseur gegangen ist.

Anstatt wie Bartsch (1978), Hartmann (1984), Posner (1972) und von Stutterheim (1989) davon auszugehen, dass Subordination grundsätzlich gewichtungsrelevant ist, zeigen die Beispiele vielmehr, dass zwei Arten von Nebensatztypen voneinander unterschieden werden müssen. Während einige Nebensätze in spezifischen Kontexten durchaus dazu verwendet werden können, die aktuell relevante Question Under Discussion zu beantworten, ist diese Möglichkeit für andere Nebensatztypen ausgeschlossen. Wenngleich Brandt (1989, 1996), Holler (2009) und Simons (2007) für einige spezifische Nebensätze beobachten, dass diese auch Hauptinformationen ausdrücken können, liegt eine systematische Untersuchung unterschiedlicher Nebensatztypen bezogen auf die Frage, ob sie dazu verwendet werden können, ohne ihren Bezugssatz die QUD zu beantworten, bislang noch nicht vor. In der vorliegenden Arbeit sollen aus diesem Grund ausgewählte Nebensatztypen diesbezüglich gestestet werden, um diese Lücke zu schließen.

Zudem wird dafür argumentiert, dass weitere zentrale Eigenschaften von abhängigen Sätzen auf ihren diskurssemantischen Status zurückgeführt werden können. Ausgangspunkt hierfür ist die Beobachtung, dass sich Nebeninformationen in einem komplexen Diskurs anders verhalten als Bedeutungsaspekte, die im Vordergrund stehen. Nebeninformationen sind von semantischen Operatoren häufig nicht betroffen, weswegen sie unter Einbettung projizieren und nicht sensibel für direkte Widerspruchsstrategien sind. Ob ein Nebensatz relevant bezogen auf die jeweilige QUD ist, beeinflusst jedoch nicht nur seine diskurssemantischen Eigenschaften, auch die Form eines Nebensatzes kann davon betroffen sein. Typischerweise besetzt das finite Verb in deutschen Nebensätzen die satzfinale Position wie in Beispiel (4-a). In spezifischen Kontexten können abhängige Sätze aber auch mit der sonst auf Hauptsätze beschränkten Verbzweit-Stellung (im Folgenden: V2-Stellung) gebildet werden (vgl. Beispiel (4-b)). Dass diese Möglichkeit jedoch nicht für alle Nebensatztypen gegeben ist, beweist das ungrammatische Beispiel (4-d).

(4) a. Peter glaubt, dass Maria den Müll runtergebracht hat.

b. Peter glaubt, Maria hat den Müll runtergebracht.

c. Peter freut sich, dass Maria den Müll runtergebracht hat.

d. *Peter freut sich, Maria hat den Müll runtergebracht.

Dass nur bestimmte Nebensätze mit V2-Stellung gebildet werden können, ist in der germanistischen Forschung bereits gut dokumentiert (vgl. beispielsweise Frank 2000, Meinunger 2004, Reis 1997 und Truckenbrodt 2006). Ein Zusammenhang mit der textstrukturellen Gewichtung des Nebensatzes wurde bislang jedoch noch nicht hergestellt, obwohl es gute Gründe gibt, einen solchen anzunehmen, wie in der vorliegenden Arbeit gezeigt wird.

Die genannten strukturellen und (diskurs)semantischen Eigenschaften von abhängigen Sätzen haben auf den ersten Blick nichts miteinander zu tun und wurden von der 
Forschung bislang auch zumeist isoliert voneinander betrachtet. ${ }^{2}$ In der vorliegenden Arbeit möchte ich dagegen für die folgende Hypothese argumentieren:

\section{HYPOTHESE}

Nur eine Untermenge aller Nebensatztypen kann dazu verwendet werden, die aktuell relevante QUD zu beantworten. Auf die derart begründete Unterscheidung von zwei Gruppen an Nebensatztypen lassen sich zentrale strukturelle und (diskurs)semantische Eigenschaften zurückführen.

Der Vorteil dieses Ansatzes gegenüber bisherigen Analysen der oben genannten strukturellen und funktionalen Eigenschaften besteht darin, dass mithilfe eines unabhängig motivierten binären Merkmals zahlreiche Aspekte von abhängigen Sätzen uniform erfasst werden können.

Der Frage, warum einige Nebensätze zur Beantwortung der aktuellen QUD verwendet werden können, andere dagegen nicht, wird am Beispiel von Temporal- und weil-Sätzen genauer nachgegangen, wobei letztere anders als Temporalsätze zum Ausdruck von Hauptinformationen geeignet sind. Hierfür wird eine genaue Beschreibung des semantischen Beitrags von temporalen Subjunktionen auf der einen und weil auf der anderen Seite vorgenommen. Die Untersuchung wird zeigen, dass sich Temporal- und weil-Sätze in der Art ihres semantischen Beitrags grundlegend voneinander unterscheiden.

Die vorliegende Arbeit ist in drei Teile gegliedert. In Teil I werden ausgesuchte Nebensatztypen, und zwar Komplementsätze von faktiven, semifaktiven und Brückenverben, Temporalsätze, weil-Sätze, appositive und restriktive Relativsätze, daraufhin untersucht, ob sie geeignet sind, die Hauptinformation auszudrücken. Zudem wird gezeigt, dass mit dem Ausdruck von Hauptinformationen weitere Eigenschaften, nämlich die Projektion unter Einbettung und die Sensibilität für direkte Widerspruchsstrategien, zusammenhängen. Im Detail geht es in Teil I um die folgenden Themen: Nach einem Überblick über die wesentlichen Annahmen fragebasierter Diskursmodelle in Abschnitt 2.1 wird in Kapitel 2.2 getestet, welche der oben genannten Nebensatztypen dazu verwendet werden können, die jeweils aktuelle QUD zu beantworten. Die auf diese Weise resultierende Klassifikation der Nebensatztypen wird in Kapitel 3 zusätzlich überprüft. Grundlegend ist hierbei die in Roberts et al. (2009) und Simons et al. (2011) begründete Annahme, dass Äußerungsteile, die nicht der Beantwortung der aktuellen QUD dienen, unter Einbettung projizieren und nicht sensibel für direkte Widerspruchsstrategien sind. Dementsprechend ist zu erwarten, dass die Nebensätze, welche im Deutschen keinen quaestiobezogenen Inhalt ausdrücken können, beide Eigenschaften aufweisen, was in Abschnitt 3.1 für Projektion und in Abschnitt 3.2 für die Widerspruchsstrategien überprüft wird. Hierbei werden

\footnotetext{
${ }^{2}$ Zwar beschäftigen sich bereits viele Arbeiten mit dem Zusammenhang zwischen der Form eines Nebensatzes und seiner Interpetation (vgl. vor allem Pasch et al. 2003 und Sweetser 1990), zumeist wurde dabei jedoch der semantische Zusammenhang zwischen dem Nebensatz und seinem Bezugssatz untersucht, wohingegen hier vor allem der funktionale Status eines Nebensatzes bezogen auf den Diskurs im Zentrum steht.
} 


\section{Einleitung}

auch die Ergebnisse einer Fragebogenstudie vorgestellt, in der die Sensibilität der oben genannten Nebensatztypen für einen direkten Widerspruch mit Nein getestet wurde. Teil I schließt in Kapitel 4 mit einer Diskussion der Frage, in welchem Verhältnis Präsupponiertheit und Nebeninformationen zueinander stehen.

Während im Mittelpunkt von Teil I funktionale, und dabei vor allem diskurssemantische Eigenschaften von Nebensätzen stehen, geht es in Teil II um die bereits oben erwähnte strukturelle Differenz zwischen Nebensätzen, die V2-Stellung aufweisen können, und solchen, welche nur mit der für Nebensätze typischen Verbletzt-Stellung (im Folgenden: VL-Stellung) wohlgeformt sind. In Kapitel 5 wird das Phänomen der V2-Nebensätze in den größeren Kontext der Wurzelphänomene eingegliedert und es wird ein kurzer Überblick über die wichtigsten Forschungsfragen des Gebiets gegeben. Anschließend werden in Kapitel 6 die oben genannten Nebensatztypen daraufhin untersucht, ob sie V2-Stellung aufweisen können. Die Frage, welche Lizenzierungsbedingungen dem Auftreten von V2-Stellung in Nebensätzen zugrunde liegen, steht im Mittelpunkt von Kapitel 7. Hier werden zuerst bisherige Ansätze diskutiert, bevor ich dann dafür argumentiere, dass es sich bei V2-Stellung in abhängigen Sätzen um eine optionale Markierung für solche Informationen handelt, welche auf die Beantwortung der jeweils aktuellen QUD abzielen. Eine kurze Diskussion von anderen (V2-)Nebensätzen und anderen Desintegrationsphänomenen des Deutschen sowie ein Vergleich mit dem Auftreten von Wurzelphänomenen im Englischen folgen in Abschnitt 7.3.

Am Beispiel des Kontrasts zwischen weil-Sätzen, welche ohne ihren Bezugssatz relevanten Inhalt bezogen auf die QUD ausdrücken können, und Temporalsätzen, für die eine solche Verwendung ausgeschlossen ist, wird in Teil III untersucht, ob dieser Unterschied auf eine tieferliegende Differenz zwischen den beiden Nebensatztypen zurückgeführt werden kann. Wir werden in Kapitel 8 sehen, dass sich Temporal- und Kausalsätze in Kopula-, quantifizierten und negierten Strukturen anders verhalten, woraus mit Johnston (1994) geschlossen wird, dass temporale Subjunktionen semantisch einstellig, kausale dagegen zweistellig sind. Eine genaue Beschreibung des semantischen Beitrags erfolgt für Temporalsätze in Abschnitt 9.1 und für weil-Sätze in Abschnitt 9.2. In diesen Abschnitten wird auch versucht, auf der Basis der jeweiligen semantischen Beschreibung zu erklären, weshalb weil-Sätze dazu geeignet sind, die aktuelle QUD zu beantworten, Temporalsätze dagegen nicht. Die Ergebnisse der Arbeit werden in Kapitel 10 zusammengefasst.

Die vorliegende Untersuchung basiert auf einem fragebasierten Diskursmodell, wie es von Klein/von Stutterheim (1992), Roberts (1996) und von Stutterheim (1989) entworfen und von Beaver/Clark (2008), Büring (2003), Roberts et al. (2009) und Simons et al. (2011) weiterentwickelt wurde (für einen Überblick vgl. Abschnitt 2.1). Für die syntaktische Beschreibung von VL- und V2-Sätzen wird eine standardgenerative Syntaxtheorie verwendet, einzelne Ergebnisse werden zudem in der Segmented Discourse Representation Theory (kurz: SDRT) nach Asher/Lascarides (2003) implementiert. 


\section{Teil I.}

\section{Diskurssemantische und -pragmatische Aspekte von abhängigen Sätzen}





\section{Zur Interpretation von Sätzen im Kontext}

Den zentralen Gegenstand der vorliegenden Arbeit bilden die (diskurs)semantischen Eigenschaften von abhängigen Sätzen. Glaubt man einer alten Schulweisheit, korreliert die zumeist strukturelle Differenz zwischen abhängigen und selbstständigen Sätzen mit einem Unterschied in der kommunikativen Funktion, da abhängige Sätze lediglich Nebengedanken enthalten dürfen, wohingegegen Hauptgedanken in selbstständigen Sätzen ausgedrückt werden. Der Gedanke, dass Haupt- von Nebengedanken zu unterscheiden sind, wurde im Rahmen neuerer Diskurstheorien formalisiert und operationalisierbar gemacht, deren Darstellung im Zentrum von Abschnitt 2.1 steht. Dabei wird die in u.a. Simons et al. (2011), Klein/von Stutterheim (1992), Roberts (1996), von Stutterheim (1989) und von Stutterheim/Klein (1989) vertretene Ansicht übernommen, dass jeder Diskurs darauf abzielt, eine explizite oder implizite Frage zu beantworten. Die Funktion von abhängigen Sätzen innerhalb eines solchen fragebasierten Diskursmodells wird in Abschnitt 2.2 anhand einiger exemplarischer abhängiger Sätze genauer untersucht, wobei gezeigt wird, dass bestimmte abhängige Sätze durchaus dazu geeignet sind, die im jeweiligen Gesprächskontext relevanteste Information auszudrücken.

\subsection{Der Kontext als Frage}

\subsubsection{Grundlagen}

Die Untersuchung von Fragen und Antworten bildet bereits seit vielen Jahren ein zentrales Gebiet der linguistischen und philosophischen Forschung (vgl. beispielsweise Altmann 1993, Bierwisch 1980, Brandt 1992, Groenendijk/Stokhof 1985, Hamblin 1958, Hamblin 1973, Higginbotham 1996, Karttunen 1977, Lohnstein 2000, Lohnstein 2013, Reis/Rosengren 1991, Reis 1992 und Truckenbrodt 2004). Fragen werden typischerweise als Interrogativsätze realisiert, wobei der Begriff des Interrogativsatzes einen morphosyntaktischen Formtypen beschreibt, wohingegen der Begriff der Frage der pragmatischen Kategorie der Illokution zuzuordnen ist. Dabei besteht keine 1:1-Abbildung zwischen Form und Funktion, da auch Satzkonstruktionen, welche formal keine Interrogativsätze sind, als Fragen verwendet werden können (vgl. für einen Überblick über die wichtigsten formalen Kriterien von Interrogativsätzen Altmann 1993 und Lohnstein 2013). So kann das folgende Beispiel aus Lohnstein (2013, 51) mit einem steigenden Tonmuster eine Frage realisieren, auch wenn es sich formal 


\section{Zur Interpretation von Sätzen im Kontext}

um einen Deklarativsatz handelt.

(5) Fritz hat ein Loch gegraben?

Daraus schließt Lohnstein (2013), dass es sich bei Interrogativität um eine semantische Kategorie handelt, wohingegen die illokutionäre Handlung des Fragens von der jeweiligen Verwendungsweise abhängt und demnach in den Bereich der Pragmatik gehört (vgl. dazu auch Bierwisch 1980). Der Illokutionstyp „Frage“ ergibt sich im Standardfall direkt aus dem semantisch-wörtlichen Konzept der Interrogativität, allerdings kann er auch wie in Beispiel (5) durch einen anderen Satzmodus ausgedrückt werden. Betrachten wir nun die Bedeutung von Fragen etwas genauer. Die zwei wichtigsten Interrogativsatztypen sind Entscheidungs- und Ergänzungsfragen wie in (6-a) und (6-b).

\section{a. Ist Homer Vegetarier? \\ b. Wer ist Vegetarier?}

Die Bedeutung von Fragen wird häufig unter Zuhilfenahme von Alternativen modelliert. Dabei wird davon ausgegangen, dass jede Frage eine Menge an Alternativen eröffnet und vom Gefragten verlangt, die zutreffende Alternative auszuwählen. Der durch einen Entscheidungsfragesatz wie (6-a) eröffnete Alternativenraum umfasst dabei zwei Elemente, nämlich das Zutreffen und das Nicht-Zutreffen des ausgedrückten Sachverhalts, er ist also binär. Dagegen eröffnet der Ergänzungsfragesatz in (6-b) einen n-fach differenzierten Alternativenraum, nämlich in diesem Fall eine Menge an Individuen (vgl. Lohnstein 2013). Nach Hamblin (1973) besteht die Bedeutung eines Fragesatzes nun genau in dieser Menge an Alternativen, also der Menge an möglichen Antworten. Die Denotation der Entscheidungsfrage (6-a) besteht demnach aus einer Menge mit zwei propositionalen Elementen, was in (7-a) und vereinfacht in (7-b) dargestellt ist:

Ist Homer Vegetarier?

a. $\quad \lambda \mathrm{p}\left[\mathrm{p}=\right.$ Vegetarier' $^{\prime}\left(\right.$ Homer' $\left.^{\prime}\right) \vee \mathrm{p}=\neg$ Vegetarier' $^{\prime}\left(\right.$ Homer' $\left.\left.^{\prime}\right)\right]$

b. $\quad\{$ Homer ist Vegetarier, Homer ist kein Vegetarier

Die Bedeutung eines Ergänzungsfragesatzes wie (6-b) lässt sich dagegen aus der Funktionalen Applikation von ist Vegetarier angewandt auf das Denotat von wer ermitteln. Anders als Personalpronomen denotiert ein Fragepronomen wie Wer nach Hamblin (1973) kein Individuum des Typs $<\mathrm{e}>$, sondern eine Menge an Individuen. Ein Fragepronomen ist demnach vom Typ $<$ e,t $>$. Nehmen wir an, Satz (6-b) wird in einem Kontext geäußert, in dem es drei relevante Individuen gibt. Dann denotiert das Fragepronomen Wer die in (8-a) dargestellte Menge, wohingegen das einstellige Prädikat ist Vegetarier das Denotat in (8-b) erhält:

a. $\llbracket$ wer' $\rrbracket=\{$ Homer, Marche, Bart $\}$

b. $\quad \llbracket$ ist Vegetarier' $\rrbracket=\left\{\lambda \mathrm{x}\left[\right.\right.$ ist $\left.\left.\operatorname{Vegetarier}^{\prime}(\mathrm{x})\right]\right\}$ 
Kombinieren wir diese beiden Denotate in Form einer Funktionalen Applikation miteinander, erhalten wir die Denotation des Ergänzungsfragesatzes (Notation nach Lohnstein 2013, 71):

$$
\begin{aligned}
& \llbracket \text { ist Vegetarier' } \rrbracket(\llbracket \text { wer' } \rrbracket) \\
& =\left\{\lambda \mathrm{x}[\text { ist Vegetarier'(x) }]\left(\text { Homer' }^{\prime}\right), \lambda \mathrm{x}[\text { ist Vegetarier'(x)](Marche'), }\right. \\
& \lambda \mathrm{x}[\text { ist Vegetarier' }(\mathrm{x})](\text { Bart') }\} \\
& =\{\text { ist Vegetarier'(Homer'), ist Vegetarier'(Marche'), ist Vegetarier'(Bart') }\}
\end{aligned}
$$

Etwas vereinfacht besteht die Denotation des Fragesatzes bezogen auf unser Modell demnach aus einer Menge mit drei Propositionen:

$$
\text { \{Homer ist Vegetarier, Marche ist Vegetarier, Bart ist Vegetarier\} }
$$

Die Reichweite des Themas reicht nun weit über die Beschäftigung mit einzelnen Fragesätzen hinaus. Von besonderem Interesse ist vor allem die diskursstrukturierende Funktion von Fragen und eine Reihe von Publikationen beschäftigen sich mit dem Einfluss von Fragen auf die Interpretation von Äußerungen. Beispielsweise wird in der Fokussemantik der Kontext, der festlegt, welche Äußerungsteile fokussiert sind und welche zum Topik gehören, häufig als Frage modelliert (so z.B. von u.a. Beaver/Clark 2008, Büring 2003, Krifka 2006, Reich 2003, Rooth 1985 und Rooth 1992). Die Äußerungsteile, welche die jeweilige Frage beantworten, bilden den Fokus einer Äußerung und werden dementsprechend sprachlich markiert. Aus diesem Grund bildet in Beispiel (11) Äußerung (11-a) eine wohlgeformte Antwort auf die Frage, nicht aber (11-b). ,\#' markiert in diesem Fall die kontextuelle Unangemessenheit der Äußerung. Demnach hängt die Interpretation von intonatorischen Fokusmarkierungen aber auch die von fokussensitiven Partikeln davon ab, welche Frage mit der jeweiligen Äußerung beantwortet werden soll.

$$
\begin{aligned}
& \text { Was hat Homer Marche gegeben? } \\
& \text { a. Homer hat ihr einen KUSS gegeben. } \\
& \text { b. \#HOmer hat ihr einen Kuss gegeben. }
\end{aligned}
$$

Neuere Ergebnisse aus der Textwissenschaft und aus der Semantik legen nun nahe, dass die diskurstrukturierende Funktion von Fragen einen noch viel größeren Einfluss hat als bisher angenommen. Die grundlegende Idee besteht dabei darin, dass Äußerungen nicht nur in Texten wie (11), in denen eine explizit gestellte Frage vorhanden ist, in ihrer Interpretation von der Frage abhängen, sondern dass jedem schriftlichen oder mündlichen Text eine explizite oder implizite Frage zugrunde liegt, welche den Inhalt und auch die Form der Äußerungen beeinflusst. Ein solches fragebasiertes Diskursmodell, demzufolge jeder Äußerungskontext als explizite oder implizite Frage verstanden werden muss, wird in diesem Abschnitt insbesondere nach Klein/von Stutterheim (1992), Roberts (1996), Simons et al. (2011), von Stutterheim (1989) und von Stutterheim/Klein (1989) erläutert, bevor es in den folgenden Abschnitten 


\section{Zur Interpretation von Sätzen im Kontext}

auf ausgewählte abhängige Sätze des Deutschen angewandt wird. ${ }^{3}$

Nach Roberts (1996) besteht das Ziel eines jeden Gesprächs oder Textes darin, Informationen auszutauschen. Dabei können zwei grundlegende Diskursaktionen voneinander unterschieden werden: Fragen etablieren ein unmittelbares Diskursziel, nämlich ihre Klärung, während Assertionen darauf abzielen, dieses Ziel zu erfüllen. Klein/von Stutterheim (1992), Roberts (1996), Roberts et al. (2009), Simons et al. (2011), von Stutterheim (1989) und von Stutterheim/Klein (1989) argumentieren nun, dass jede assertive Äußerung vor dem Hintergrund einer Frage, mit dem Ziel, diese zu beantworten, getätigt wird. Diese Frage kann explizit als Äußerung im Kontext gegeben sein, in den meisten Fällen bleibt sie jedoch implizit und muss rekonstruiert werden. Diese Frage, die Question Under Discussion (kurz: QUD) bei Roberts (1996) und Simons et al. (2011), bzw. die Quaestio in der Terminologie von von Stutterheim (1989), bildet den Kontext, vor dessen Hintergrund jede assertive Äußerung interpretiert werden muss. In seiner Gesamtheit dient ein Text dann jeweils der Beantwortung dieser expliziten oder impliziten Frage. Die einem Diskurs zugrundeliegende Frage wird im Folgenden nach Büring (2003) als Q bezeichnet.

Ein Diskurs wird demnach immer durch eine oder auch mehrere, seriell aufeinander folgende Fragen strukturiert. Akzeptieren die Gesprächsteilnehmer eine Frage $\mathrm{Q}$ und deren Beantwortung als Diskursziel, unterliegt jede weitere Äußerung dem Gebot, eine relevante Äußerung bezogen auf Q zu machen, und zwar so lange, bis Q beantwortet ist oder es sich herausgestellt hat, dass Q unbeantwortbar ist. Die jeweilis aktuelle Q strukturiert einen Diskurs demnach, indem sie bestimmt, was gesagt wird, sie beeinflusst aber auch die sprachliche Form, in der bestimmte Inhalte geäußert werden können, was Gegenstand der folgenden Abschnitte sein wird.

Die Art der einem Diskurs zugrundeliegenden Frage variiert mit der Textsorte. Von Stutterheim (1989) beobachtet, dass bestimmte Textsorten wie Wegauskünfte oder Argumentationen der Beantwortung einer klar umrissenen Frage dienen, wohingegen andere Textsorten häufig mehrere Fragen hintereinander beantworten (vgl. auch Klein/von Stutterheim 1992, 69). Als charakteristische Quaestio für Erzählungen formulieren Klein/von Stutterheim $(1992,69)$ die folgende Frage: ${ }^{4}$

Was ist (dir) zum Zeitpunkt $\mathrm{x}$ am Ort y passiert?

Die den Diskurskontext konstituierende Frage kann aber auch enger gefasst sein wie beispielsweise $(13) .^{5}$

\footnotetext{
${ }^{3}$ Dabei ist zu berücksichtigen, dass es sich bei dem fragebasierten Diskursmodell um eine Theorie in der Ausarbeitung handelt und einzelne Aspekte noch nicht ausreichend untersucht wurden. Im Folgenden wird auf die Stellen, an denen eine bislang ausstehende theoretische Präzisierung die Untersuchung beeinträchtigt, hingewiesen.

${ }^{4}$ Allerdings spekulieren Klein/von Stutterheim (1992, 69), dass bestimmte Textsorten wie etwa Small-talk gar keine bestimmte Quaestio verfolgen.

${ }^{5}$ An diesem Beispiel zeigt sich, dass Q und die tatsächlich geäußerte Frage häufig nicht identisch sind. Möchte ein Sprecher S allgemein etwas über den Verlauf des Betriebsausflugs erfahren, so würde er dies wahrscheinlich eher mit der folgenden Frage realisieren anstatt mit (13):
}

Wie war der Betriebsausflug? 
(13) Was ist beim Betriebsausflug passiert?

Nach Büring (2003) besteht die einzige Beschränkung für Q darin, dass mit Q nichts offensichtlich Bekanntes, im Common Ground bereits Gegebenes erfragt werden darf. Ist die Beantwortung von $\mathrm{Q}$ einmal als Diskursziel akzeptiert, dient jede weitere Äußerung der Erfüllung dieses Ziels. Der gesamte Diskurs in seinem Aufbau wird nun durch die jeweils zugrundeliegende Frage bestimmt, wodurch ein Diskurs erst kohärent wird. Nehmen wir einmal an, Frage (13) entspricht Q. Um Q zu beantworten, können die Diskursteilnehmer auch erst einmal eine Unterfrage, eine sogenannte SubQ, aufwerfen und diese beantworten. Beispielsweise kann geklärt werden, wer an dem Ausflug teilgenommen hat, was das Ausflugsziel war und wie das Wetter gewesen ist. Die Aufspaltung der übergeordneten Q in Unterfragen kann in Form eines Baumdiagramms, einem sogenannten Diskursbaum oder D-tree in der Terminologie von Büring (2003), dargestellt werden, wie in Grafik 2.1 veranschaulicht (vgl. dazu Beaver/Clark 2008, Büring 2003, Büring 2007 und Roberts 1996). Die Abbildung zeigt, dass die jeweilige Q das einem Diskurs zugrundeliegende Organisationsprinzip bildet. Zudem spiegelt die Abbildung wider, wie sich ein Diskurs in mehrere, hintereinander geschaltete Fragen aufspalten kann.

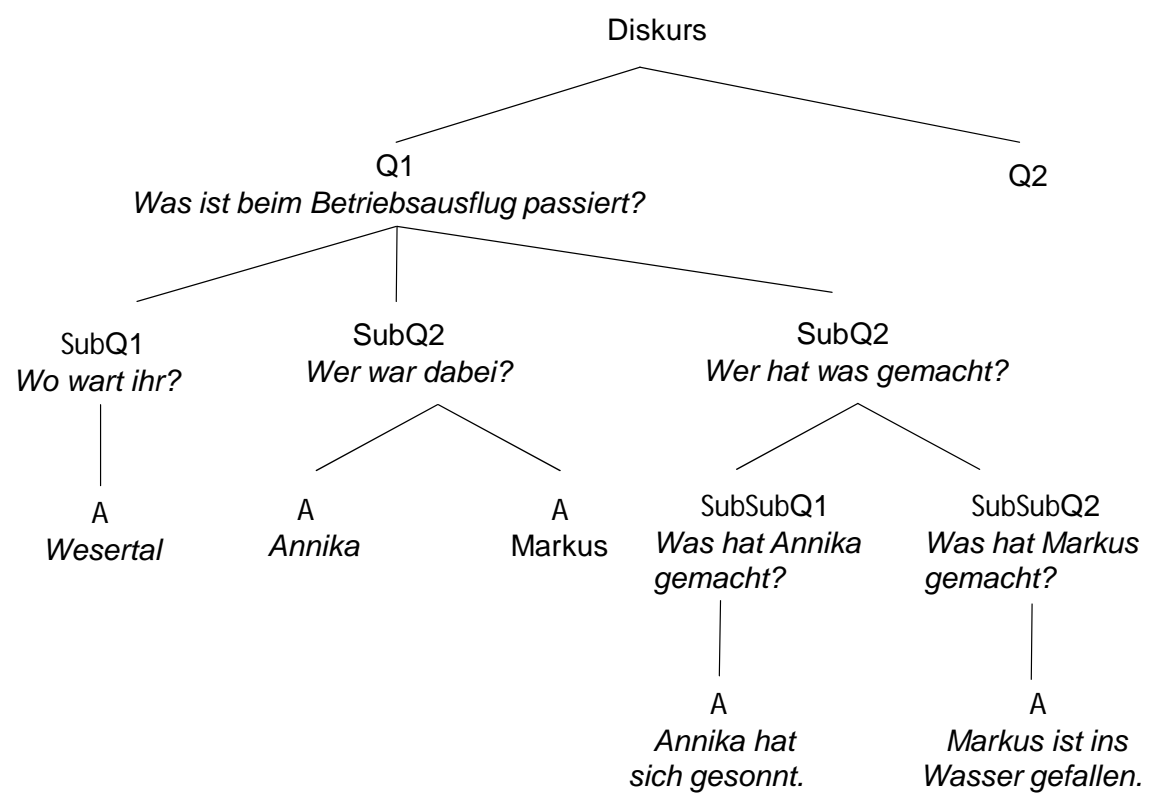

Abbildung 2.1.: Exemplarischer Diskursbaum

Klein/von Stutterheim $(1992,69)$ gehen in diesem Zusammenhang davon aus, dass die Gesprächsteilnehmer Q umdefinieren können. 
Wir haben zu Beginn dieses Abschnitts gesehen, dass Fragesätze Alternativen eröffnen. Hieraus ergeben sich nun Konsequenzen für die Annahme, dass jeder Diskurs als Ziel die gemeinsame Beantwortung einer Frage Q hat. Die Beantwortung einer Frage besteht darin, aus der Menge an Alternativen die zutreffende auszuwählen und alle anderen auszuschließen. Dabei kann eine Frage durch eine Äußerung komplett oder partiell beantwortet werden. Nach Roberts (1996) schließt eine komplette Antwort alle möglichen Alternativen bis auf eine aus. B's Äußerung in dem folgenden Beispiel bildet eine solche komplette Antwort.

$$
\begin{aligned}
& \text { A: Wer ist Vegetarier? } \\
& \text { B: Marche und sonst niemand. } \\
& \text { B': Marche. (+> und sonst niemand) }
\end{aligned}
$$

Die durch die Frage eröffnete Alternativenmenge ist bezogen auf ein beliebig festgelegtes Modell mit drei Individuen in (10) dargestellt. Aus dieser Menge wählt B eine Alternative und drückt aus, dass die anderen Alternativen nicht zutreffend sind. Aber auch die Antwort von B' kann als komplette Antwort fungieren. Die Äußerung schließt aufgrund ihrer wörtlichen Bedeutung die beiden anderen Alternativen Homer ist Vegetarier und Bart ist Vegetarier zwar nicht aus. Mithilfe der Quantitätsmaxime kann sich A jedoch die Exhaustivitätsimplikatur ableiten, derzufolge alle anderen Alternativen nicht zutreffend sind. Eine partielle Antwort dagegen schließt mindestens eine Alternative aus, lässt das Zutreffen der übrigen Alternativen jedoch offen. Ein Beispiel hierfür wäre die Äußerung B".

$$
\begin{aligned}
& \text { A: Wer ist Vegetarier? } \\
& \text { B": Homer zumindest nicht. }
\end{aligned}
$$

Wird Q, wie in Abbildung 2.1 dargestellt, in Unterfragen unterteilt, so gilt, dass eine komplette Antwort auf eine Unterfrage Q' einer partiellen Beantwortung von Q entspricht, wie Onea (2011) und Roberts (1996) beobachten. Dies wird in dem folgenden Beispiel deutlich: Hier bildet Q' eine Unterfrage von Q. Wie wir sehen, wird Q' durch A' komplett und Q partiell beantwortet. Umgekehrt bildet eine komplette Antwort auf Q auch eine komplette Beantwortung aller Unterfragen von Q. Dies ist mit der Äußerung A illustriert, welche sowohl Q als auch Q' komplett beantwortet.

$$
\begin{aligned}
& \text { Q: Wer ist Vegetarier? } \\
& \text { Q': Ist Homer Vegetarier? } \\
& \text { A': Nein, Homer ist kein Vegetarier. } \\
& \text { A: Homer ist Vegetarier und sonst niemand. }
\end{aligned}
$$

Ähnliche Annahmen formuliert Higginbotham (1996) im Rahmen seines Partitionsansatzes. Anders als bei Hamblin (1973) ist die durch einen Fragesatz eröffnete Alternativenmenge bei Higginbotham (1996) in Klassen zerlegt. Dies ermöglicht die Bewertung der Informativität einer Antwort (vgl. dazu Lohnstein 2013). Etwas vereinfacht gilt dabei (Notation nach Lohnstein 2013, 77): 


\section{Verfahren zur Bestimmung der Informativität einer Antwort:}

Sei $\Pi$ der von einer Frage denotierte Antwortraum und \# die Kardinalität einer Menge. Eine Antwort A auf eine Frage $\Pi$ ist

i. vollständig, wenn $\#(\Pi / \mathrm{A})=1$.

ii. partiell, wenn $1<\#(\Pi / \mathrm{A})<\mathrm{n}$.

iii. irrelevant, wenn $\#(\Pi / \mathrm{A})=\mathrm{n}$.

Auch hier gilt, dass eine komplette Antwort vorliegt, wenn die Alternativenmenge durch sie auf genau ein Element reduziert wird. Dagegen reduziert eine partielle Antwort zwar die Antwortmenge, es bleiben aber immer noch mindestens zwei Alternativen übrig. Irrelevant bezogen auf eine Frage ist eine Äußerung dann, wenn durch sie keine einzige Alternative aus der Antwortmenge ausgeschlossen werden kann und die Alternativenmenge demnach nicht reduziert wird.

Nach Roberts (1996) bleibt eine Frage Q solange aktuell, bis sie komplett beantwortet ist, d.h. alle bis auf eine Alternativen ausgeschlossen wurden, oder sie sich nach Ansicht der Diskursteilnehmer als unbeantwortbar herausstellt. Solange dies noch nicht der Fall ist, müssen alle Sprechakte darauf abzielen, Q zu lösen, indem durch sie mindestens eine partielle Antwort realisiert wird. Zudem ist das Aufstellen einer neuen Frage Q', solange Q noch nicht komplett beantwortet ist, nur dann erlaubt, wenn es sich dabei um eine Unterfrage von Q handelt und die Beantwortung von Q' bezogen auf Q relevant ist (vgl. Abbildung 2.1). ${ }^{6}$ Demnach unterliegen auch Fragen einer Relevanzanforderung.

Unterfragen bilden aber nicht die einzige Art von Fragen, welche relevant bezogen auf $\mathrm{Q}$ sind. Manchmal kann die Beantwortung von $\mathrm{Q}$ vorangetrieben werden, indem eine weitere Frage aufgestellt wird wie in dem folgenden Beispiel. In diesem Fall ergibt sich die Beantwortung von Q aus der Beantwortung von B's Frage. Das Beispiel zeigt, dass nicht nur die jeweils aktuell gegebene Q die Äußerungen in einem Gespräch beeinflusst, sondern dass diese auch Rückwirkungen auf Q haben können, indem sie Q modifizieren.

A: Ist Silke schon im Urlaub?

B: Haben die Schulferien schon angefangen?

Analysiert man den Kontext, wie in diesem Abschnitt vorgestellt, als Frage, müssen nicht nur weitere Fragen, sondern auch Assertionen eine Relevanzanforderung erfüllen, indem sie zumindest immer eine partielle Antwort auf die aktuelle Q liefern. Auf diese Weise rekonstruiert bereits Grice (1989) Implikaturen, die durch Befolgung der Relationsmaxime entstehen. Beispielsweise kann A aus B's Äußerung die Implikatur ableiten, dass möglicherweise ihr Hund das Steak gegessen hat, Q wird also indirekt beantwortet.

$$
\text { A: Wo ist denn mein Steak? }
$$

\footnotetext{
${ }^{6}$ In Abschnitt 2.1.2 wird jedoch gezeigt, dass Teile der sogenannten Nebenstruktur eine eigene, von der dem Diskurs zugrundeliegenden QUD unabhängige Frage beantworten können, wie u.a. Onea (2013) zeigt.
} 
B: Dein Hund sieht ganz zufrieden aus.

Aus der Relevanzanforderung an Assertionen ergibt sich ein weiterer Punkt, nämlich die Tatsache, dass das Auftreten von Assertionen auf bestimmte Kontexte beschränkt ist. Beispielsweise bildet der Satz Peter ist Vegetarier. als Antwort auf Q oder Q' eine wohlgeformte Äußerung, nicht aber in einem Kontext wie Q".

Q: Wer ist Vegetarier?

Q': Ist Peter Vegetarier?

Q": Wie ist das Wetter?

Daraus schließt Onea (2011), dass jede deklarative Äußerung bestimmte Anforderungen an den Kontext, in dem sie auftreten kann, stellt. Die Tatsache, dass jede deklarative Äußerung Selektionsbeschränkungen für Kontexte beinhaltet, modelliert Onea (2011) als Präsupposition. Die Äußerung Peter ist Vegetarier. präsupponiert beispielsweise, dass die zu beantwortende Frage Wer ist Vegetarier? oder Ist Peter Vegetarier? lautet, aber nicht Wie ist das Wetter?. Diese Präsupposition muss im Kontext gesättigt werden, andernfalls ist die Äußerung im jeweiligen Kontext nicht angemessen. An der Tatsache, dass der Satz jedoch in mindestens zwei unterschiedlichen Kontexten auftreten kann, sieht man bereits, dass der Kontext durch die Selektionseigenschaften einer deklarativen Äußerung nicht genau bestimmt, sondern unterspezifiziert ist. Wesentlich ist jedoch, dass bestimmte Kontexte wie beispielsweise Q" in (20) ausgeschlossen werden, oder zumindest ohne pragmatische Anreicherung nicht direkt zugänglich sind.

Werden pragmatische Reinterpretationen in die Betrachtung miteinbezogen, kann ein Satz in deutlich mehr Kontexten geäußert werden, als in den entsprechenden Präsuppositionseigenschaften vorgesehen ist. So wird in dem folgenden Beispiel eine Äußerung in einem Kontext verwendet, in dem ihre Präsupposition eindeutig nicht gesättigt ist, um zu signalisieren, dass der Sprecher nicht über das entsprechende Thema reden möchte.

(21) A: Herr Wulff, erzählen Sie unseren Lesern die Hintergründe der Kreditaffäre?

B: Oh, es hat angefangen zu regnen.

Derartige Fälle werden seit Grice (1989) als Implikaturen analysiert, welche aus einem Verstoß gegen die Relevanzmaxime resultieren. Den Annahmen Oneas (2011) folgend können wir dagegen spekulieren, ob nicht vielmehr ein Verstoß gegen die Präsuppositionsanforderungen des Deklarativsatzes vorliegt, welcher dann pragmatisch reinterpretiert wird.

Die Präsupposition eines Satzes, durch die die Art des Kontextes, in dem der Satz geäußert werden kann, spezifiziert wird, bezeichnet demnach die rein semantische Ebene. Auf der semantischen Ebene wird entschieden, ob die Proposition eines Satzes relevant bezogen auf die jeweilige Q ist. Pragmatische Reinterpretationen sind dagegen auch in solchen Fällen möglich, in denen ein Satz auf der semantischen 
Ebene im entsprechenden Kontext keine angemessene Äußerung bildet. Es ist also entscheidend, zwischen einem semantischen und einem pragmatischen Antwortbegriff zu unterscheiden. Wird im Folgenden ein Satz als kontextuell unangemessene Äußerung mit dem Zeichen ,\#' markiert, wird damit lediglich die semantische Ebene bezeichnet, wohl wissend, dass häufig eine pragmatische Umdeutung möglich ist.

Wir haben gesehen, dass ein und derselbe Deklarativsatz als Antwort auf unterschiedliche Fragen verwendet werden kann, die entsprechende Frage in seinen Präsuppositionsanforderungen demnach unterspezifiziert ist. Nun kann aber mithilfe sprachlicher Markierungen ein Indiz darauf gegeben werden, wie die jeweils zu beantwortende Q lautet. Auf diese sprachlichen Markierungen wird in Abschnitt 2.1.3 und in Teil II genauer eingegangen. Zuvor jedoch werden wir sehen, dass es auch Äußerungen gibt, welche nicht auf die Beantwortung von Q abzielen und nach von Stutterheim (1989) die Nebenstruktur eines Diskurses bilden.

\subsubsection{Haupt- und Nebenstruktur}

Klein/von Stutterheim (1992) und von Stutterheim (1989) beobachten, dass nicht alle in einem Diskurs gemachten Äußerungen und Äußerungsteile der Beantwortung der jeweiligen $\mathrm{Q}$ dienen, sondern auch andere Funktionen haben können, wie zum Beispiel Bewertungen oder Kommentare auszudrücken, oder zusätzliche Beschreibungen, Erklärungen und andere Hintergrundinformationen zu liefern. Ein Text besteht deswegen immer aus zwei Ebenen, einer Ebene an Äußerungen, die auf die Beantwortung von Q abzielen, und zusätzlich Äußerungen, welche darüber hinaus weitere Informationen liefern. Von Stutterheim (1989) spricht in diesem Zusammenhang von Haupt- und Nebenstruktur eines Textes. Eine andere Terminologie für den im Prinzip gleichen Sachverhalt benutzen Roberts et al. (2009) und Simons et al. (2011), welche Äußerungen und Äußerungsteile, die die aktuelle Q beantworten, als [+at-issue]-Inhalte bezeichnen, wohingegen alle anderen Äußerungen [-at-issue] sind. Im Folgenden werde ich die deutschen Begriffe Haupt- und Nebenstruktur synonym zu den Begriffen [+at-issue] und [-at-issue] verwenden.

Betrachten wir ein Beispiel aus von Stutterheim $(1989,181)$ zur Unterscheidung zwischen Haupt- und Nebenstruktur, und zwar einen Ausschnitt aus einer Erzählung. Wesentliches Merkmal einer Erzählung ist nach Klein/von Stutterheim (1992, 69ff) die Tatsache, dass bestimmte Teilereignisse erzählt werden, welche Element einer übergeordneten Ereignisstruktur sind und in einer zeitlichen Abfolge zueinander stehen. Diese Beschreibung passt auf die Sätze (22-a) und (22-c) des folgenden Beispiels, nicht aber auf Satz (22-b), der demnach der Nebenstruktur zuzuordnen ist.

a. Klefisch verließ das Haus um sieben.

b. Gewöhnlich fuhr er mit dem Bus ins Büro.

c. Heute aber nahm er ein Taxi.

Das wesentliche Merkmal zur Unterscheidung zwischen Haupt- und Nebenstruktur in Erzählungen wie (22) besteht nach von Stutterheim (1989) darin, dass Äuße- 


\section{Zur Interpretation von Sätzen im Kontext}

rungen der Nebenstruktur keine Verschiebung auf der Zeitachse bewirken, sie für die chronologische Abfolge also unerheblich sind. Dieses Merkmal eignet sich jedoch nicht für alle Textsorten als Unterscheidungskriterium zwischen Haupt- und Nebenstruktur. So weist von Stutterheim (1989) selbst darauf hin, dass die chronologische Abfolge von Teilereignissen in nicht-narrativen Texten keine Rolle spielt. Betrachten wir dazu das folgende Beispiel aus Klein/von Stutterheim (1992, 73). Gegeben ist Q als Kontext. In diesem Fall dienen die kursivierten Äußerungen bzw. Äußerungsteile nicht der Beantwortung von Q, sondern liefern als Teil der Nebenstruktur zusätzliche Informationen (Kursivierung nach Klein/von Stutterheim 1992, 73). Äußerungen der Nebenstruktur kann dabei durchaus eine eigene Frage zugrundeliegen, worauf am Ende dieses Abschnitts noch genauer eingegangen wird - beispielsweise beantwortet der zweite kursivierte Äußerungsteil die implizite Frage Ist der Platz schön?.

Q: Können Sie mir sagen, wo das Goethehaus ist?

A: Ja, lassen Sie mich einen Moment überlegen. Ich war selbst letzte Woche dort. Ja, Sie gehen hier ungefähr 300 Meter runter, dann gehen Sie hinter der Kirche links. Dann nochmal nach 300 Metern kommen Sie zu einem Platz, einem sehr schönen Platz. Den überqueren Sie, gehen weiter und gehen dann nach rechts. Sie können es wirklich nicht verfehlen. Dann ist es die zweite Straße links, und dann sehen Sie es schon. Es ist gelb, so gelblich. Okay?

Da in diesem Fall keine chronologische Abfolge erkennbar ist, bleibt als Kriterium zur Unterscheidung zwischen Haupt- und Nebenstruktur lediglich die Frage, ob die jeweilige Äußerung der Beantwortung von Q dient. Dieses Kriterium ist natürlich besonders in den Fällen problematisch, in denen Q implizit ist.

Ganz ähnlich definieren Simons et al. (2011) [+at-issue]-Inhalte im Gegensatz zu solchen Inhalten, die [-at-issue], also nicht Teil der Hauptstruktur, sind. Auch bei Simons et al. (2011) ist für die Definition von At-issueness das Konzept der Relevanz bezogen auf die jeweilige Q entscheidend. Dabei gilt nach Simons et al. (2011, 8):

\section{Relevance to the QUD}

a. An assertion is relevant to a QUD iff it contextually entails a partial or complete answer to the QUD.

b. A question is relevant to a QUD iff it has an answer which contextually entails a partial or complete answer to the QUD.

Anstatt At-issueness direkt für eine Proposition p zu bestimmen, schlagen Simons et al. (2011) vor, die Zugehörigkeit zur Hauptstruktur indirekt über die damit verbundene Entscheidungsfrage ?p zu definieren. ?p bezeichnet dabei bezogen auf eine Proposition p die Frage, ob p, das heißt, eine Aufteilung der Menge an Welten in solche, in denen $\mathrm{p}$ gilt, und solche, in denen $\neg$ p gilt. Die Zugehörigkeit zur Hauptstruktur definieren Simons et al. (2011) dann wie folgt: 


\section{Definition von At-issueness}

A proposition $\mathrm{p}$ is at-issue relative to a question $\mathrm{Q}$ iff ?p is relevant to $\mathrm{Q}$.

Betrachten wir mit dieser Definition im Hinterkopf noch einmal Beispiel (23). Nach Klein/von Stutterheim $(1992,73)$ ist der Satz $\mathrm{p}=$ Ich war selbst letzte Woche dort. nicht Teil der Hauptstruktur, wenn $\mathrm{Q}=$ Wie komme ich von hier zum Goethehaus? lautet. Auch nach der Definition von Simons et al. (2011) ist p nicht at-issue, da die Beantwortung der Frage $? \mathrm{p}=$ War A letzte Woche im Goethehaus? keine relevante Information für die Beantwortung von Q enthält. Genauer gesagt führt die Beantwortung von ?p zu keiner Reduktion der durch Q eröffneten Alternativenmenge. Nach der Bestimmung der Relevanz einer Äußerung bezogen auf eine Frage nach Higginbotham (1996) (vgl. (17)) liegt demnach eine irrelevante Äußerung vor, da die Zahl der in der Alternativenmenge enthaltenen Alternativen gleich bleibt (es gilt also Fall iii. aus (17), d.h. \#(П/A) = n). Das von Simons et al. (2011) vorgeschlagene Testverfahren liefert in diesem Fall demnach das gleiche Ergebnis, zu dem auch Klein/von Stutterheim (1992) kommen.

Kombinieren wir die Definition von At-issueness in (25) nach Simons et al. (2011) mit dem Verfahren zur Bestimmung der Informativität einer Antwort nach Higginbotham (1996) (vgl. (17)), erhalten wir die folgenden Zuordnungen:

\section{Zuordnung einer Proposition zur Haupt- oder Nebenstruktur:}

i. $\quad$ Eine Proposition ist in einem Kontext, in dem $\mathrm{Q}=\Pi$, genau dann Teil der Hauptstruktur, wenn $\#(\Pi / \mathrm{A})=1$ oder $1<\#(\Pi / \mathrm{A})<\mathrm{n}$.

ii. Eine Proposition ist in einem Kontext, in $\operatorname{dem} \mathrm{Q}=\Pi$, genau dann Teil der Nebenstruktur, wenn \#(П/A) = n.

Dies bedeutet, dass eine Proposition p nur dann [+at-issue]-Inhalt ausdrückt, wenn die durch Q eröffnete Alternativenmenge durch die Äußerung von p reduziert wird. Bleibt die Anzahl der ursprünglichen Alternativen gleicht, können wir dagegen davon ausgehen, dass p [-at-issue]-Inhalt enthält.

Die Behauptung, dass [-at-issue]-Inhalte anders als [+at-issue]-Inhalte nicht der Beantwortung einer Frage dienen, wurde in der letzten Zeit jedoch häufiger angefochten. So zeigen Beaver (2012), Fabricius-Hansen (2013), Holler (2005) und Onea (2013) bezogen auf die diskursstrukturelle Funktion von appositiven Relativsätzen, dass diese eine eigene Frage beantworten und aus diesem Grund Ähnlichkeiten mit selbstständigen Sätzen aufweisen können, beispielsweise indem sie Fokusmarkierungen enthalten, worauf im folgenden Abschnitt noch genauer eingegangen wird. Allerdings handelt es sich dabei nicht um die dem Diskurs zugrundeliegende QUD und auch um keine Unterfrage, vielmehr beantworten appositive Relativsätze eine eigene Frage, über die Fabricius-Hansen (2013) aussagt, dass sie lokal relevant, aber nicht relevant bezogen auf die globale QUD ist. Beaver (2012) spricht in diesem Zusammenhang von einer Non-Under Discussion Question, kurz Non-UDQ, wobei dieser Begriff auch im Folgenden verwendet wird. Dass appositiven Relativsätzen 


\section{Zur Interpretation von Sätzen im Kontext}

eine Frage zugrundeliegt, deren Beantwortung für den weiteren Diskursverlauf nicht relevant ist, zeigt sich auch darin, dass ihr Inhalt im Folgediskurs gewöhnlich nicht aufgegriffen oder weitergeführt wird, wie Onea (2013) beobachtet. Beispielsweise signalisiert der Sprecher mit einer Äußerung wie (27-a), dass er nicht die Absicht hat, den im Relativsatz ausgedrückten Inhalt zum Gegenstand des weiteren Diskurses zu machen, weswegen die Weiterführung in (27-a), wohl aber die in (27-b), nicht angemessen ist. Die Beantwortung der N-UDQ Seit wann kennst du Maria? in Beispiel (27-a) ist auf diese Weise in den Diskurs eingebaut, ohne den Haupterzählstrang zu stören.

a. Morgen gehe ich mit Maria, die ich übrigens schon seit dem Kindergarten kenne, ins Kino. ??Damals konnten wir uns aber nicht ausstehen.

b. Morgen gehe ich mit Maria ins Kino. Ich kenne sie übrigens schon seit dem Kindergarten. Damals konnten wir uns aber nicht ausstehen.

Bei der von appositiven Relativsätzen beantworteten N-UDQ handelt es sich typischerweise um eine vom Matrixsatz derivierte Frage, Holler (2005) formuliert eine mögliche N-UDQ mit Welche Eigenschaften hat $x$ noch?, eine andere denkbare Möglichkeit wäre Wie ist meine persönliche Einstellung zu x?. Onea (2013) führt diesbezüglich den Begriff der Potential Questions ein, worunter solche Fragen fallen, die durch getätigte Assertionen erzeugt werden können. Laut Onea (2013) sind alle Fragen, deren Präsuppositionen durch die bereits getätigten Assertionen erfüllt sind, mögliche Potential Questions.

Dass appositive Relativsätze der Beantwortung einer N-UDQ dienen, ist weitgehend akzeptiert, ob dies jedoch grundsätzlich für alle [-at-issue]-Inhalte gilt, ist von der Forschung noch zu untersuchen. Aber auch wenn einige oder möglicherweise sogar alle [-at-issue]-Inhalte der Beantwortung einer eigenen Frage dienen, sind sie dennoch Teil der Nebenstruktur. Am Beispiel der appositiven Relativsätze wird deutlich, dass deren Inhalt im Folgediskurs nicht weiter aufgegriffen werden soll, zudem weisen appositive Relativsätze typische Eigenschaften der Nebenstruktur auf, worauf in Kapitel 3 noch genauer eingegangen wird.

Die Zugehörigkeit zur Hauptstruktur wird immer in Relation zu Q bestimmt. Problematisch ist nun allerdings, dass die jeweils aktuelle Q in einem schriftlichen oder mündlichen Text sehr häufig nicht explizit gegeben ist. Daraus ergeben sich im Wesentlichen zwei Fragen: (i) Mithilfe welcher Strategien können sich die Diskursteilnehmer Q rekonstruieren? und (ii) Wie können wir testen, ob eine Äußerung oder ein Äußerungsteil zur Haupt- oder zur Nebenstruktur gehört, wenn Q implizit ist? Ob eine Äußerung Teil der Hauptstruktur ist, hängt zunächst einmal vom Diskurskontext und der entsprechenden Q ab. Dies bedeutet, dass für eine Äußerung wie (28) kontextlos nicht bestimmt werden kann, ob diese zur Haupt- oder zur Nebenstruktur gehört.

Tim isst kein Fleisch. 
Handelt es sich um eine Antwort auf die Frage Isst Tim Fleisch?, dann gehört Satz (28) zweifelsohne zur Hauptstruktur. Wird der Satz dagegen in dem folgenden Kontext geäußert, ist sein Inhalt nicht relevant bezogen auf die aktuelle $\mathrm{Q}$ und demnach auch nicht Teil der Hauptstruktur.

Q: Was hast du gestern gemacht?

A: Ich war einkaufen und habe dann gekocht. Eigentlich wollte ich Steaks braten, aber Tim isst kein Fleisch. Deswegen habe ich dann Gemüselasagne gemacht.

Zudem kann aus einer Äußerung wie (28) ohne Kontext nicht ohne Weiteres rekonstruiert werden, wie die zu beantwortende Q lautet. Satz (28) liefert beispielsweise eine Antwort auf die Fragen in (30) und auf viele weitere.
a. Wer isst kein Fleisch?
b. Worauf verzichtet Tim?
c. Was hast du gerade erfahren?

Allerdings beobachten Onea (2011) und von Stutterheim (1989), dass uns die Grammatik anhand sprachlicher Mittel Hinweise darauf gibt, ob eine Äußerung relevant zur Beantwortung von $\mathrm{Q}$ ist oder nicht, und so auch indirekt Hinweise darauf, wie die zu beantwortende Q lautet. Die Art dieser sprachlichen Markierung ist Gegenstand des folgenden Abschnitts.

Auch in der germanistischen Relieftheorie wird zwischen kommunikativ relevanten Haupt- und kommunikativ nicht-relevanten Nebengedanken unterschieden (vgl. Bartsch 1978, Brandt 1989, Brandt 1996, Hartmann 1984, Posner 1972 und Weinrich 1964). Im Unterschied zu dem hier vorgestellten fragebasierten Diskursmodell ist die Differenzierung zwischen Haupt- und Nebeninformationen in diesen Ansätzen jedoch intuitiver und in einem geringerem Maße operationalisierbar, da von keiner dem Diskurs zugrundeliegenden Frage ausgegangen wird. Es wird zwar ebenfalls angenommen, dass die kommunikative Relevanz eines Satzes nur relativ zu einem Kontext beschrieben werden kann, der Kontext als solcher bleibt aber anders als in fragebasierten Modellen, wo der Kontext einem durch eine Frage eröffneten Alternativenraum entspricht, ein relativ vager Begriff. Eine Gemeinsamkeit zwischen Relieftheorie und dem hier vorgestellten fragebasierten Diskursmodell besteht jedoch darin, dass in beiden Ansätzen davon ausgegangen wird, dass die Informationsgewichtung anhand sprachlicher Mittel ausgedrückt wird, worauf im Folgenden eingegangen wird.

\subsubsection{Sprachliche Markierung}

Im vorherigen Abschnitt haben wir gesehen, dass für einen Satz wie (28) ohne Kontext nicht entschieden werden kann, ob er zur Haupt- oder Nebenstruktur gehört, geschweige denn, welche Art von Q mit der Äußerung beantwortet wird, falls diese zur Hauptstruktur gehört. Ergänzen wir die Äußerung (28) nun um ein Fokusmerkmal, 


\section{Zur Interpretation von Sätzen im Kontext}

welches im Deutschen durch einen Pitch-Akzent realisiert wird, wird die Anzahl der möglichen Fragen, die durch die Äußerung beantwortet werden können, eingegrenzt. Der folgende Beispielsatz liefert zwar immer noch eine Antwort auf die beiden Fragen (30-b) und (30-c), er kann jedoch nicht mehr in einem Kontext geäußert werden, in $\operatorname{dem} \mathrm{Q}=$ Wer isst kein Fleisch?

$$
\text { Tim isst kein }\left[\text { FLEISCH }_{F}\right] \text {. }
$$

Frage und Fokussierung sind immer komplementär zueinander, indem eine Konstituente, welche ein Fokusmerkmal trägt, mit einem W-Wort in der entsprechenden Frage übereinstimmen muss. Aus diesem Grund bildet Satz (31) keine angemessene Antwort auf die Frage Wer isst kein Fleisch?. ${ }^{7}$ Das Fokusmerkmal gibt folglich einen Hinweis darauf, wie die zu beantwortende Q lautet. Da der fokussierten Konstituente ein W-Ausdruck in der zu beantwortenden Frage entsprechen muss, kann Q auf diese Weise rekonstruiert werden. Dies ist insbesondere in Fällen, in denen Q implizit ist, relevant. Zudem lässt sich feststellen, dass nur [+at-issue]-Inhalte fokussiert werden können. Aus diesem Grund ist das folgende Beispiel, in dem die mit einem Fokusmerkmal versehene Äußerung Teil der Nebenstruktur ist, nicht wohlgeformt:

Q: Was hast du gestern gemacht?

B: Ich war einkaufen und habe dann gekocht. \#Eigentlich wollte ich Steaks

\footnotetext{
${ }^{7}$ In Abschnitt 2.1.1 haben wir gesehen, dass die Denotation eines Fragesatzes aus einer Menge an Alternativen besteht. Auch die Semantik von Fokussierungen wird mithilfe von Alternativen beschrieben (vgl. Büring 2003, Rooth 1985, Rooth 1992 und von Stechow 1990 sowie Kadmon 2001, 227ff und Krifka 2008 für einen Überblick). Die Grundidee der Alternativensemantik besteht darin, dass fokussierte Ausdrücke neben ihrer Standardbedeutung einen zweiten, fokussemantischen Wert besitzen. Beispielsweise denotiert ein prosodisch markierter Fokusträger wie in Beispiel (31) zusätzlich zu seiner gewöhnlichen Bedeutung eine Menge an Alternativen. Die Konstituente, welche den Fokusakzent erhält, ist dabei Träger eines Fokusmerkmals F. Das Fokusmerkmal zeigt an, dass zu seinem Träger eine Menge an Alternativen existiert, durch welche der fokussierte Ausdruck potentiell substituiert werden könnte, und dass der fokussierte Ausdruck als ein Element dieser Alternativenmenge entnommen wurde. Um die gewöhnliche Intension eines Ausdrucks $\alpha$ wiederzugeben, wird die Notation $\llbracket \alpha \rrbracket^{0}$ verwendet, wohingegen $\llbracket \alpha \rrbracket^{f}$ seine fokussemantische Bedeutung ausdrückt, also eine Menge an Alternativen. Der fokussierte Ausdruck Fleisch führt beispielsweise eine Alternativenmenge wie in (i) dargestellt ein. Die jeweilige Alternativenmenge wird dabei im Wesentlichen durch Faktoren wie Kontext und Relevanz definiert.
}

$\llbracket$ Fleisch $\rrbracket^{f}=\{$ Fisch, Obst, Gemüse, $\ldots\}$

Nicht-fokussierte Ausdrücke evozieren dagegen keine Alternativen. Fokussierung hat demnach eine einem Fragesatz verwandte Funktion, indem in beiden Fällen auf eine Menge an Alternativen verwiesen wird. Aus diesem Grund existieren auch Ansätze, die Interaktion zwischen Fokus- und wh-Merkmal syntaktisch abzubilden (vgl. beispielsweise Grewendorf 2002 und Sabel 2006). Ein zentraler Unterschied zwischen Fragen und Fokussierung besteht nach Lohnstein $(2013,78)$ aber darin, dass mit der Äußerung einer Frage eine Alternativenmenge eröffnet und vom Adressaten erwartet wird, diese Menge bis auf eine Alternative zu reduzieren. Dagegen ist es in Fokuskonstruktionen der Sprecher selbst, der die zutreffende Alternative benennt und alle anderen Alternativen ausschließt. 
braten, aber Tim isst kein FLEISCH ${ }_{F}$. Deswegen habe ich dann Gemüselasagne gemacht.

Da ein fokussierter Ausdruck immer einem W-Ausdruck entsprechen muss, kann er folglich auch nie [-at-issue] sein.

Am Beispiel der Fokussierung wird deutlich, dass anhand sprachlicher Mittel angezeigt werden kann, ob eine Äußerung oder ein Äußerungsteil [+at-issue]- oder [-atissue]-Inhalt ausdrückt. Ein Fokusmerkmal signalisiert die Zugehörigkeit zur Hauptstruktur und demnach gehen mit ihm auch Selektionsbeschränkungen für mögliche Kontexte einher. So beobachtet Onea (2011), dass ein fokussierter Ausdruck nur in bestimmten Kontexten verwendet werden kann, da durch das Fokusmerkmal der Typ der vorhergehenden Frage präsupponiert wird. Wir können also zwei Dinge festhalten, die eng miteinander zusammenhängen: (i) Anhand sprachlicher Mittel kann signalisiert werden, ob eine Äußerung bzw. ein Äußerungsteil zur Haupt- oder zur Nebenstruktur gehört. (ii) Gehört eine Äußerung zur Hauptstruktur, werden anhand sprachlicher Mittel Hinweise darauf gegeben, wie die Frage Q lautet, die die Äußerung zu beantworten sucht. Daran, dass Satz (31) immer noch als Antwort auf mehrere Fragen geeignet ist, wird jedoch auch ersichtlich, dass Q anhand derartiger sprachlicher Mittel nicht eindeutig markiert wird. Auch bei einem Satz wie (31) ist Q unterspezifiziert und muss kontextuell erschlossen werden. Somit liegt keine 1:1Entsprechung zwischen dem Aussehen von Q und ihrer grammatischen Markierung vor. Zentral ist jedoch, dass es sprachliche Mittel wie die Fokusintonation in Beispiel (31) ermöglichen, einige mögliche Fragen auszuschließen. ${ }^{8}$

Neben Fokusintonation existieren weitere Mittel, um die Zugehörigkeit zur Hauptoder Nebenstruktur zu markieren. Onea (2011) macht beispielsweise darauf aufmerksam, dass die Verwendung des definiten Artikels ein Signal dafür ist, dass die Existenz der entsprechenden Entität nicht zur Debatte steht, und auch in der germanistischen Forschung zur Reliefbildung wird davon ausgegangen, dass die kommunikative Gewichtung anhand sprachlicher Mittel gekennzeichnet wird. ${ }^{9}$ So untersucht Hartmann (1984) die reliefbildende Funktion von Modalpartikeln und weist darauf hin, dass Parenthesen nur zum Ausdruck von Nebeninformationen geeignet sind. Zu einem ähnlichen Schluss kommt auch Brandt $(1989,1996)$, die zeigt, dass der Inhalt von Parenthesen nie quaestiobezogen ist.

Wenn es um die sprachliche Markierung von (Nicht-)At-issueness geht, ist es wichtig, zwischen einem semantischen und einem pragmatischen Antwortbegriff zu unter-

\footnotetext{
${ }^{8}$ Die Aussage, dass nur [+at-issue]-Inhalte Fokusmerkmale tragen können, muss allerdings eingeschränkt werden. In Abschnitt 2.1.2 wurde bereits gezeigt, dass [-at-issue]-Inhalte wie appositive Relativsätze eine N-UDQ beantworten können. In diesem Fall kann dieser Teil der Nebenstruktur auch fokussierte Teile enthalten wie in dem folgenden Beispiel:

(i) Q: Wo warst du?

B: Ich war mit Tim, der blöderweise kein FLEISCH $_{F}$ isst, im Steakhaus.

${ }^{9}$ Hartmann (1984) argumentiert zudem, dass auch nicht-sprachliche Mittel der Reliefgebung dienen können.
} 


\section{Zur Interpretation von Sätzen im Kontext}

scheiden (vgl. dazu u.a. Beispiel (21)). Eine Äußerung oder eine Teiläußerung kann sprachlich als Teil der Nebenstruktur markiert sein, aber dennoch relevanten Inhalt bezogen auf die QUD ausdrücken, worauf in Kapitel 2.2 noch anhand zahlreicher Beispiele eingegangen wird.

Auch die syntaktische Unterscheidung von Haupt- und Nebensatz wird häufig als Mittel zur kommunikativen Gewichtung betrachtet. Beispielsweise geht von Stutterheim $(1989,168)$ davon aus, dass Nebensätze nie relevante Informationen bezogen auf die jeweilige $\mathrm{Q}$ beinhalten. Damit folgt sie einer langen Tradition, welche in der alten schulgrammatischen Stilregel von Reiners (1951, 102) als „Hauptsachen in Hauptsätze, Nebensachen in Nebensätze" zusammengefasst wird. Auch in der germanistischen Literatur zur Reliefgebung wird die kommunikative Relevanz von komplexen Sätzen untersucht. Genau wie von Stutterheim (1989) kommen Bartsch (1978), Hartmann (1984) und Posner (1972) dabei zu dem Schluss, dass dem Unterschied zwischen Haupt- und Nebensatz die Differenz zwischen Haupt- und Nebeninformation entspricht. Da in diesen Texten angenommen wird, dass nur Hauptsätze Träger des kontextuell relevanten Inhalts sein können, wird geschlossen, dass die strukturelle Distinktion zwischen Haupt- und Nebensatz eindeutig die kommunikative Gewichtung markiert. Diese Annahme wird durch die Beobachtung gestützt, dass die Verwendung eines Temporalsatzes in einem Kontext, in dem er ohne seinen Bezugssatz die bezogen auf $\mathrm{Q}$ relevante Information ausdrückt, nicht angemessen ist, wie das folgende Beispiel belegt: ${ }^{10}$

Q: War Peter gestern im Kino?

B: \#Peter hat seine Mutter besucht, nachdem er im Kino war.

Der Ansicht, die kommunikative Gewichtung sei eindeutig durch die syntaktische Unterscheidung von Haupt- und Nebensatz bestimmt, widerspricht allerdings Holler (2009), die zeigt, dass auch weil-Sätze und weiterführende Relativsätze alleinige Träger der Hauptinformation sein können. ${ }^{11}$ Auch Brandt $(1989,1996)$ kommt zu dem Ergebnis, dass die Gliederung in Haupt- und Nebensätze kein eindeutiges

${ }^{10}$ Die Verwendung eines Temporalsatzes im gleichen Kontext ist dagegen absolut wohlgeformt, wenn Q zuvor beispielsweise mit einem $J a$ beantwortet wird:

(i) Q: War Peter gestern im Kino?

B: Ja, Peter hat seine Mutter besucht, nachdem er im Kino war.

In diesem Fall ist der Inhalt des Temporalsatzes zum Äußerungszeitpunkt nicht mehr Teil der Hauptstruktur, da die relevante Q ja schon geklärt ist (vgl. auch Beispiel (65) in Abschnitt $2.2 .3)$.

${ }^{11}$ Statt aus der syntaktischen Struktur leitet Holler (2009) die Informationsgewichtung aus der diskurssemantischen Textstrukturierung im Rahmen der Segmented Discourse Representation Theory nach Asher/Lascarides (2003) ab. Entscheidend ist hierbei, dass Äußerungen entweder mit einer neben- oder einer Hierarchie stiftenden unterordnenden Diskursrelation mit dem vorhergehenden Diskurs verknüpft werden können. Wird ein Nebensatz nun über eine nebenordnende Relation wie beispielsweise $\mathrm{R}=$ Continuation angebunden, drückt er Hauptinformationen aus, andernfalls gehört er zur Nebenstruktur. 
Mittel zur Signalisierung von At-issueness ist. ${ }^{12}$ Dass auch Nebensätze ohne ihren Bezugssatz Träger von [+at-issue]-Inhalt sein können, belegt das folgende Beispiel, in dem der restriktive Relativsatz die für die Beantwortung von Q relevante Information ausdrückt (vgl. für ähnliche Beispiele aus dem Englischen und Norwegischen Simons 2007 und Wiklund et al. 2009).

Q: Welche Qualifikationen müssen Bewerber mitbringen?

B: Die Firma sucht einen Mitarbeiter, der Chinesisch kann.

Wir können demnach festhalten, dass es sich bei syntaktischer Subordination im Deutschen nicht um ein Mittel zur Markierung von [-at-issue]-Inhalten handelt. Beispiel (33) hat aber auch gezeigt, dass zwei Arten von Nebensatztypen voneinander unterschieden werden müssen, wenn es um die quaestiobezogene Gewichtung geht. Ob ein Nebensatz geeignet ist, ohne seinen Bezugssatz die zur Beantwortung von Q relevante Information auszudrücken, hängt wesentlich vom Nebensatztyp ab. Die Verwendung eines Temporalsatzes ist, wie in Beispiel (33) gezeigt, nicht wohlgeformt, wenn der Satz ohne seinen Bezugssatz Teil der Hauptstruktur ist, wohingegen dies für restriktive Relativsätze möglich ist. Daraus ergibt sich die Frage nach dem Verhalten anderer Nebensätze. Dementsprechend werden in Abschnitt 2.2 einige ausgesuchte Nebensätze exemplarisch daraufhin untersucht, ob sie ohne ihren Bezugssatz [+at-issue]-Inhalt ausdrücken können.

\subsubsection{Zusammenfassung}

Die wichtigsten Punkte des in diesem Abschnitt geleisteten Überblicks über das im Folgenden verwendete fragebasierte Diskursmodell werden im Folgenden noch einmal kurz zusammengefasst.

- Ein Diskurs dient in der Regel der Beantwortung einer expliziten oder impliziten Frage Q. Ist die Beantwortung von Q durch die Gesprächsteilnehmer als Diskursziel akzeptiert, unterliegen alle weiteren Äußerungen einer Relevanzanforderung bezogen auf dieses Ziel.

\footnotetext{
${ }^{12}$ Stattdessen schlägt Brandt (1989, 1996) eine zweidimensionale Reliefgebung vor, bei der sie zwischen kommunikativer und quaestiobezogener Gewichtung unterscheidet. Dabei argumentiert sie, dass Subordination bei bestimmten Nebensätzen der kommunikativen Gewichtung dient, indem S weniger wichtige Informationen herunterstufen kann. Basierend auf dem vor allem phonologisch begründeten Begriff der Informationseinheit unterscheidet sie zwei Arten von Nebensätzen. Bei Nebensätzen, die eine eigene Informationseinheit bilden, ist die Subordination gewichtungsrelevant, da die im Nebensatz ausgedrückte Informationseinheit im Verhältnis zum Matrixsatz heruntergestuft ist. Nebensätze dagegen, die mit ihrem Matrixsatz zusammen eine Informationseinheit bilden, können auch die zentrale Information enthalten, hier ist die Subordination demnach nicht gewichtungsrelevant. Etwas problematisch ist dabei, dass der Begriff der phonologisch basierten Informationseinheit fragwürdig ist und auch die Distinktion von kommunikativer und quaestiobezogener Gewichtung ist zweifelhaft, da ein systematischer Zusammenhang zwischen beiden besteht, wie Holler (2009) bemerkt.
} 
- Relevanzanforderung für deklarative Äußerungen: Jede deklarative Äußerung muss mindestens eine partielle Antwort auf Q enthalten, d.h. den durch Q eröffneten Alternativenraum mindestens um eine Alternative reduzieren.

- Relevanzanforderung für Fragen: Solange Q noch nicht komplett beantwortet ist, muss jede neue Frage Q' eine Unterfrage von Q sein, oder die Beantwortung von Q' muss auf eine andere Weise der Beantwortung von Q beitragen.

- Ausgenommen von der Relevanzanforderung sind Äußerungen der Nebenstruktur, welche bezogen auf Q keine relevante Information enthalten, sondern Kommentare, Begründungen oder Hintergrundinformationen ausdrücken. Die jeweils aktuelle Q bestimmt den Aufbau eines Diskurses, indem in Relation zu Q festgelegt wird, welche Äußerungen zur Haupt-, und welche zur Nebenstruktur gehören. Hierbei ist es jedoch wichtig, zwischen einem semantischen und einem pragmatischen Antwortbegriff zu unterscheiden.

- Die jeweilige Q beeinflusst die Ausdrucksweise, da die Zugehörigkeit zur Hauptoder Nebenstruktur mit der Verwendung bestimmter sprachlicher Mittel signalisiert wird.

- Indem die Zugehörigkeit zur Haupt- oder Nebenstruktur sprachlich markiert wird, werden auch Hinweise darauf geliefert, wie die jeweilige Q lautet. Allerdings wird Q aufgrund solcher Markierungen nie eindeutig bestimmt, sondern ist unterspezifiziert und muss kontextuell erschlossen werden.

- [-At-issue]-Inhalte können eine eigene Frage, eine Non-Under Discussion Question, beantworten und können dann auch Fokusmarkierungen aufweisen.

- Bei der strukturellen Unterscheidung von Haupt- und Nebensätzen handelt es sich um kein eindeutiges Mittel zur Markierung von [+at-issue]- und [-atissue]-Inhalten.

\subsection{Abhängige Sätze und die Beantwortung von $Q$}

\subsubsection{Grundlagen}

In ihrer Diskussion darüber, mit welchen sprachlichen Mitteln im Deutschen Hauptund Nebenstruktur markiert werden, geht von Stutterheim $(1989,168)$ davon aus, „dass Äußerungen, die zum Vordergrund zählen, (...) Hauptsätze sein müssen “ und auch Bartsch (1978) und Hartmann (1984) argumentieren, dass die Informationsgewichtung durch die Unterscheidung von Haupt- und Nebensatz eindeutig gesteuert wird. Dass Nebensätze aber nicht ausschließlich [-at-issue]-Inhalte ausdrücken, haben bereits Brandt (1989, 1996) und Holler (2009) gezeigt und auch Beispiel (34) belegt, dass ein Nebensatz auch ohne seinen Bezugssatz die zur Beantwortung von 
Q relevante Information enthalten kann. Wir haben aber auch gesehen, dass Temporalsätze, anders als der Relativsatz in (34), offensichtlich nicht ohne ihren Bezugssatz [+at-issue]-Inhalt ausdrücken können. In diesem Abschnitt sollen nun ausgesuchte Nebensätze daraufhin untersucht werden, ob sie dazu geeignet sind, ohne ihren Bezugssatz relevante Informationen bezogen auf eine vorgegebene QUD auszudrücken. Wie bei Reich/Reis (2013) wird der Begriff des Neben- oder auch abhängigen Satzes im Folgenden als Überbegriff für integrierte Gliedsätze und unintegrierte Nebensätze verwendet. Hauptsätze bezeichnen dagegen selbstständige Sätze. In den folgenden Abschnitten werden vor allem integrierte Nebensätze untersucht, welche Reich/Reis (2013, 537) folgendermaßen definieren:

S2 ist genau dann in S1 integriert (eingebettet), wenn der minimale Satzknoten von S1 den maximalen Satzknoten von S2 dominiert.

Zudem zeichnen sich integrierte Nebensätze dadurch aus, dass sie VL-Stellung aufweisen, durch eine unterordnende Subjunktion eingeleitet werden, Quantoren-VariablenBindung zulassen und entweder syntaktisch selegiert sind (Komplementsätze) oder aber ihren Bezugssatz oder einen Teilausdruck daraus modifizieren (Adverbial- und Attributsätze). ${ }^{13}$ Während in Teil I der vorliegenden Arbeit vor allem die diskurssemantischen Eigenschaften integrierter Nebensätze untersucht werden (vgl. dazu die Abschnitte 2.2.2 bis 3.3 sowie 4.2, eine Ausnahme bilden appositive Relativsätze), stehen im Zentrum von Teil II mit abhängigen V2-Strukturen unintegrierte Nebensätze, welche sich nach Reich/Reis (2013) dadurch auszeichnen, dass sie an ihren Bezugssatz adjungiert sind, keine Quantoren-Variablen-Bindung zulassen, nicht im Skopus von Elementen aus dem Bezugssatz stehen, nicht einbettbar sind und eine separate Fokus-Hintergrund-Gliederung (kurz: FHG) aufweisen. Zudem können unintegrierte Nebensätze weggelassen werden, ohne dass dies ihren Bezugssatz syntaktisch oder semantisch beeinträchtigt (vgl. für eine ausführlichere Diskussion von unintegrierten Nebensätzen Teil III).

Einzelne Nebensätze wurden bezogen auf die Frage, ob sie ohne ihren Bezugssatz [+at-issue]-Inhalt ausdrücken können, von der Forschung bereits diskutiert, beispielsweise untersucht Holler (2005, 2009) die quaestiobezogene Verwendung von weiterführenden und anderen nicht-restriktiven Relativsätzen. Eine systematische Untersuchung der Funktion von unterschiedlichen Nebensätzen bezogen auf die Diskursgliederung in Haupt- und Nebenstruktur steht bislang jedoch noch aus. Deswegen werden in diesem Abschnitt die acht folgenden Nebensätze daraufhin getestet, ob sie alleinige Träger von [+at-issue]-Inhalten sein können: Komplementsätze von faktiven, semifaktiven und Brückenverben, mit weil eingeleitete Adverbialsätze, temporale Adverbialsätze, appositive Relativsätze sowie restriktive Relativsätze mit definitem und indefinitem Antezedens. Natürlich handelt es sich hierbei nur um eine nicht vollständige Auswahl, es werden aber die drei wichtigen Nebensatztypgruppen Adverbialsatz, Komplement- und Relativsatz exemplarisch abgedeckt. Eine

\footnotetext{
${ }^{13}$ Dass eine solche Klassifikation nicht unproblematisch ist, zeigen Reich/Reis (2013) anhand zahlreicher Beispiele.
} 


\section{Zur Interpretation von Sätzen im Kontext}

umfassendere, systematische Untersuchung weiterer Nebensatztypen ist für zukünftige Forschungen sicherlich wünschenswert. ${ }^{14}$

In (25) haben wir gesehen, dass Simons et al. $(2011,8)$ das Konzept der At-issueness bezogen auf einen Satz p indirekt über die entsprechende Entscheidungsfrage ?p definieren. Bezogen auf einen Nebensatz bedeutet dies, dass p sowie die Entscheidungsfrage ?p unter Wegfall der nebensatzeinleitenden Subjunktion gebildet werden, wie in (36) illustiert:

Peter hat seine Mutter besucht, nachdem er im Kino war.

$\mathrm{p}=$ Peter war im Kino.

$? \mathrm{p}=$ War Peter im Kino?

Je nachdem, ob die Beantwortung von ?p relevant bezogen auf die jeweilige Q ist, gehört p entweder zur Haupt- oder Nebenstruktur. Genauer gesagt haben wir gesehen, dass die Klärung der Bipartition p oder $\neg \mathrm{p}$ zu einer Reduktion der durch $\mathrm{Q}$ eröffneten Alternativenmenge um mindestens ein Element führen muss, damit p [+at-issue]-Inhalt ausdrückt (es muss also \#(П/A) $=1$ oder $1<\#(\Pi / \mathrm{A})<\mathrm{n}$ gelten, vgl. die Zuordnungen in (26)). Führt die Beantwortung von ?p dagegen zu keiner Reduktion des Alternativenraums von Q, ist p bezogen auf Q [-at-issue] und somit Teil der Nebenstruktur (es gilt demnach Fall ii. aus (26), d.h. \#(П/A) =n). Die Relevanz einer Äußerung p und der damit verbundenen Entscheidungsfrage ?p kann immer nur bezogen auf eine kontextuell gegebene Frage Q bestimmt werden. Aus diesem Grund werden für die folgende Untersuchung die Kontexte ganz genau definiert, indem $\mathrm{Q}$ als explizit gestellte Frage angegeben ist. Die natürlichsprachliche Frage entspricht also jeweils der aktuell relevanten Q. Die oben genannten Nebensatztypen werden dann jeweils in zwei unterschiedlichen Kontexten getestet: Zum einen als Antwort auf eine Frage, in Bezug auf die sie nicht relevant sind, zum anderen in einem Kontext, in dem der Nebensatz gemäß der Definition (25) [+atissue]-Inhalt ausdrückt. Zu erwarten ist dabei, dass alle Nebensatztypen in Kontext 1 als Teil der Nebenstruktur verwendet werden können, in Kontext 2, als Teil der Hauptstruktur, dagegen nur manche Nebensatztypen geäußert werden können. Bei der Untersuchung, welche Nebensätze dazu verwendet werden können, ohne ihren Bezugssatz [+at-issue]-Inhalt auszudrücken, wird die Nebensatzproposition auf der semantischen Ebene betrachtet, pragmatische Umdeutungen wie in Beispiel (21) werden außer Acht gelassen.

Im Folgenden wird nur dann davon ausgegangen, dass ein Nebensatz [+at-issue]Inhalt ausdrückt, wenn er alleine, d.h. ohne den Informationsgehalt seines Bezugssatzes, die relevante Information bezogen auf Q ausdrückt. Dieser Fall ist klar zu unterscheiden von Beispielen, in denen Haupt- und Nebensatz zusammen die aktuel-

\footnotetext{
${ }^{14}$ Bezogen auf das Norwegische untersuchen Bentzen (2009) und Wiklund et al. (2009) für Komplement- und zu einem geringerem Maße auch für Adverbialsätze, ob sie die zur Beantwortung einer gestellten Frage relevante Information enthalten können, und kommen dabei zu ganz ähnlichen Ergebnissen, wie sie in den folgenden Abschnitten für das Deutsche ermittelt werden. Nicht betrachtet werden in den Untersuchungen von Bentzen (2009) und Wiklund et al. (2009) Relativsätze.
} 
le Q beantworten. Beispielsweise bildet in dem folgenden Kontext die Gesamtäußerung B eine relevante Antwort bezogen auf Q und der eingebettete Satz ist Teil des [+at-issue]-Inhalts. Für sich gesehen, ist der Nebensatz aber [-at-issue].

Q: Warum ist Marche so aufgebracht?

B: Homer glaubt dem Bäcker, dass Kuchen gesund ist.

Dies ist vergleichbar mit der Analyse des Illokutionspotentials von eingebetteten Sätzen. So ist der Komplementsatz in dem folgenden Beispiel natürlich Teil der Assertion Homer bedauert $x$, für sich gesehen ist die Nebensatzproposition jedoch präsupponiert.

Homer bedauert, dass er den Zaun kaputt gemacht hat.

\subsubsection{Komplementsätze von Verben}

Beginnen wir mit Nebensätzen, die eine Argumentforderung des Matrixprädikats erfüllen und zu deren quaestiobezogener Gewichtung bislang keine systematische Untersuchung vorliegt. Bezogen auf die Frage, ob ein Komplementsatz [+at-issue]Inhalt ausdrücken kann, während sein Bezugssatz Teil der Nebenstruktur ist, spielt die Art des Matrixprädikats eine entscheidende Rolle. Verben, welche als Objekt einen finiten Nebensatz selegieren, können aufgrund ihrer semantischen und syntaktischen Eigenschaften in unterschiedliche Klassen aufgeteilt werden. Die wichtigsten Klassen sind in (39) mit jeweils einigen Beispielen aufgelistet. Daneben existieren weitere Klassen, wie etwa Präferenz- oder Gewissheitsprädikate, auf die in der folgenden Untersuchung jedoch nicht weiter eingegangen wird (zur Klassifikation von nebensatzeinbettenden Prädikaten vgl. u.a. Frank 2000, Hooper/Thompson 1973, Karttunen 1971, Kiparsky/Kiparsky 1970, Meinunger 2004 und Reis 1997).

i. Sagensprädikate (verba dicendi): sagen, erzählen, behaupten

ii. Einstellungsprädikate (verba putandi): glauben, hoffen, finden

iii. Semifaktive Prädikate: entdecken, wissen, erfahren

iv. Berücksichtigungsprädikate: ignorieren, beachten, vernachlässigen

v. Emotive Verben: bereuen, übelnehmen, beklagen

Die Sagens- und Einstellungsprädikate bilden zusammen die Gruppe der Brückenverben (vgl. u.a. Meinunger 2004), welche sich vor allem durch die Möglichkeit der Extraktion aus dem Komplementsatz auszeichnen. So ist es möglich, das Objekt eines durch ein Brückenverb eingebetteten Satzes zu erfragen (vgl. (40-a)), diese Form der langen Extraktion ist aber ungrammatisch bei Nicht-Brückenverben wie in Beispiel (40-b) (vgl. zur Extraktion aus Komplementsätzen auch Grewendorf 1988, Müller 2011 und Reis 1994).

(40) a. Wem 1 glaubt Evi, dass sie $t_{1}$ das Buch geben muss?

b. ${ }^{*} \mathrm{Wem}_{1}$ beachtet Evi, dass sie $\mathrm{t}_{1}$ das Buch geben muss? 


\section{Zur Interpretation von Sätzen im Kontext}

Zudem können Verben der Gruppen i. und ii. einen Nebensatz selegieren, welcher ein assertives Illokutionspotential besitzt (vgl. dazu vor allem Hooper/Thompson 1973 und Reis 1997 sowie Abschnitt 7.1). Im Deutschen können solche assertiven Komplementsätze unter Wegfall des Komplementierers die sonst auf Hauptsätze beschränkte V2-Stellung aufweisen, was im Zentrum von Teil II stehen wird:

Maria hat gesagt, Peter fliegt diesen Sommer nach Japan.

Prädikate der Gruppe iii., also semifaktive Verben, präsupponieren typischerweise ihr Komplement. So wird mit der Äußerung von Satz (42-a) beispielsweise das Zutreffen der Nebensatzproposition vorausgesetzt. Diese Inferenz bleibt auch erhalten, wenn der Matrixsatz wie in (42-b) negiert wird.

a. Jenny hat erfahren, dass Evi die Schuhe gekauft hat.

b. Jenny hat noch nicht erfahren, dass Evi die Schuhe gekauft hat.

$\gg$ Evi hat die Schuhe gekauft.

Anders als faktive Verben verlieren semifaktive Verben jedoch ihre Faktivität, wenn sie Teil eines Fragesatzes oder eines Konditionalsatzgefüges sind, wie u.a. Hooper/ Thompson $(1973,480)$ und Karttunen (1971) beobachten. Beispielsweise kann Jenny zu Evi, nachdem letztere den Schuhkauf dementiert hat, den folgenden Satz sagen, ohne dass der Komplementsatz hier als wahr präsupponiert würde (vgl. zum Projektionsverhalten der unterschiedlichen Nebensatztypen auch Abschnitt 3.1).

(43) Sollte ich später erfahren, dass du die Schuhe gekauft hast, gibt's 'nen Mordsärger.

Die Verbklassen iv. - v. schließlich gehören zu den faktiven Verben, welche wie semifaktive Verben ihr Komplement präsupponieren. Anders als bei semifaktiven Verben geht die Faktivität dieser Verben aber auch in Frage- und Konditionalgefügen nicht verloren, wie das folgende Beispiel illustriert. Auch in das Antezedens eines Konditionalsatzes eingebettet, ist das Zutreffen des Objektsatzes präsupponiert.

Sollte ich später bereuen, dass ich die Schuhe gekauft habe, kann ich sie ja umtauschen.

Betrachten wir nun die einzelnen Verbklassen bezogen auf den diskurssemantischen Status des durch sie eingebetteten Satzes, beginnend mit Klasse i., den Sagensprädikaten. In dem folgenden Beispiel ist die Klärung der Frage ?p = Ist Bier gesund? nicht relevant für die Beantwortung von Q. Gemäß der Defition (25) nach Simons et al. (2011) drückt der Nebensatz demnach [-at-issue]-Inhalt aus, da die Klärung von ?p zu keiner Reduktion der durch Q eröffneten Alternativenmenge führt. Es gilt folglich Fall ii. aus Definition (26), da $\#(\Pi / A)=$ n, und demnach gehört der Komplementsatz zur Nebenstruktur. Wie zu erwarten, ist die Äußerung in diesem Kontext wohlgeformt.

Q: Wer erzählt denn so einen Unsinn? 
B: Homer hat gesagt, dass Bier gesund ist.

Dasselbe gilt nun auch für einen Kontext, in dem der Komplementsatz gemäß der Definition (25) Teil der Hauptstruktur ist, wohingegen der Hauptsatz [-at-issue]Inhalt ausdrückt. In Beispiel (46) trägt ?p = Muss Marche heute länger arbeiten? zu der Beantwortung von Q bei.

Q: Wo ist Marche?

B: Homer hat gesagt, dass sie heute länger arbeiten muss.

In diesem Beispiel eröffnet Q eine Menge an Alternativen, welche unterschiedliche Orte nennen, an denen Marche sein könnte. Die Denotation des W-Worts ist in (47) abgebildet:

$$
\llbracket \text { wo’ } \rrbracket=\{\text { im Büro, im Supermarkt, bei den Nachbarn }\}
$$

Durch Klärung von ?p wird diese Alternativenmenge nun bis auf ein Element reduziert, nach Higginbotham (1996) liegt demnach eine vollständige Antwort vor, da $\#(\Pi / \mathrm{A})=1$ (vgl. Fall i. in $(26))$. Demnach drückt der Komplementsatz in diesem Kontext [+at-issue]-Inhalt aus. Wie wir sehen, ist die Äußerung in diesem Kontext wohlgeformt, woraus wir schließen können, dass die Komplemente von Sagensprädikaten Teil der Hauptstruktur sein können. Auch wenn der Komplementsatz in diesem Kontext eine vollständige Antwort bezogen auf Q ausdrückt, ist jedoch zu beachten, dass er unter einem Sagensprädikat eingebettet ist. Somit verpflichtet sich Sprecher B weniger stark auf das Zutreffen von p, als wenn p uneingebettet auftreten würde. ${ }^{15}$ Ganz ähnlich verhält es sich mit durch Einstellungsprädikate eingebetteten Sätzen, die ebenfalls je nach Kontext entweder zur Haupt- oder zur Nebenstruktur gehören können. In dem folgenden Beispiel liefert die eingebettete Proposition keine Antwort auf $\mathrm{Q}$, da die Beantwortung der entsprechenden Frage ?p = Hat Marche Homer betrogen? zu keiner Reduktion der durch Q eröffneten Alternativenmenge führt. Q wird in diesem Fall vielmehr durch die Gesamtäußerung B's beantwortet, nicht aber durch den Nebensatz alleine.

$$
\begin{aligned}
& \text { Q: Warum ist Marche so traurig? } \\
& \text { B: Homer glaubt, dass sie ihn betrogen hat. }
\end{aligned}
$$

${ }^{15}$ Ein Parameter, der in der vorliegenden Untersuchung unberücksichtigt bleibt, betrifft die Position des Nebensatzes. Ist ein nicht-faktiver Komplementsatz topikalisiert wie in dem folgenden Beispiel, kann er nicht Träger der Hauptinformation sein:

(i) Q: Wo ist Marche?

B: \#Dass sie heute länger arbeiten muss, hat Homer gesagt.

Eine ähnliche Beobachtung haben bereits Bartsch (1978) und Hartmann (1984) gemacht, die beide davon ausgehen, dass das Vorfeld im Deutschen die Position für das bereits eingeführte Thema ist. Im Folgenden werden nur nachgestellte Nebensätze untersucht. 
Zudem können die Komplemente von Einstellungsprädikaten genau wie Komplementsätze von Sagensprädikaten ohne ihren Bezugssatz Teil der Hauptstruktur sein wie in Kurzdialog (49).

Q: Wo ist Marche?

B: Homer glaubt, dass sie heute länger arbeiten muss.

Wir können also festhalten, dass Verben der Gruppen i. und ii. ein Komplement selegieren, dass entweder Teil der Haupt- oder der Nebenstruktur sein kann.

Wie verhalten sich nun die Komplemente von semifaktiven Verben? Stellen wir uns vor, das folgende Frage-Antwort-Paar wird in einem Kontext geäußert, in dem es zum Common Ground gehört, dass Bart Raucher ist. In diesem Fall gehört der eingebettete Satz zur Nebenstruktur.

Q: Was ist passiert?

B: Marche hat entdeckt, dass Bart raucht.

Bezogen auf die Frage, ob durch semifaktive Verben wie entdecken oder realisieren eingebettete Sätze auch für die Beantwortung von Q relevante Informationen enthalten können, herrschen in der Forschungsliteratur unterschiedliche Ansichten. Simons et al. $(2011,10)$ führen das folgende Beispiel an, um zu zeigen, dass semifaktive Komplementsätze nicht [+at-issue] sein können (Bewertung des Beispiels nach Simons et al. 2011, 10).

Q: What's the weather like?

B: \#Bob realizes that it's raining.

Allerdings wirkt die Äußerung deutlich besser, wenn der Satz etwas verändert wird wie in (52):

Q: Wie ist denn das Wetter?

B: Ach, Bob hat gerade aus dem Fenster geguckt und mit Missmut realisiert, dass es mal wieder regnet.

Dass das Komplement eines semifaktiven Verbs ohne seinen Bezugssatz [+at-issue]Inhalt ausdrücken kann, zeigt sich noch deutlicher, wenn statt des Verbs realisieren ein anderes semifaktives Verb verwendet wird. In dem folgenden Kurzdialog ist die Klärung von ?p = Muss Marche heute länger arbeiten? relevant bezogen auf Q und die Gesamtäußerung wohlgeformt.

Q: Wo ist denn Marche?

B: Homer hat gerade erfahren, dass sie heute länger arbeiten muss.

Auch Simons $(2007,1035)$ geht davon aus, dass ein Verb wie to discover einen zur Klärung von Q relevanten Nebensatz einbetten kann, und belegt dies mit dem 
folgenden Beispiel: ${ }^{16}$

Q: Where was Harriet yesterday?

B: Henry discovered that she had a job interview at Princeton.

Der Nebensatz ist im Sinne der Definition (25) eindeutig relevant bezogen auf Q, wohingegen der Matrixsatz nach Simons $(2007,1035)$ eine rein evidentiale Funktion ausübt, indem er die Quelle der Information benennt. Nichtsdestotrotz ist die Äußerung absolut wohlgeformt, woraus wir schließen können, dass auch semifaktive Komplementsätze ohne ihren Bezugssatz Träger von [+at-issue]-Inhalten sein können.

Soweit können wir festhalten, dass die Verben der Gruppen i., ii. und iii. Nebensätze einbetten, die entweder zur Haupt- oder zur Nebenstruktur gehören. Wesentlich ist dabei der Kontext, der über den diskurssemantischen Status des eingebetteten Satzes entscheidet.

Kommen wir nun zu den Gruppen iv. und v., den faktiven Verben. In dem folgenden Beispiel ist der Inhalt des durch das Berücksichtigungsprädikat ignorieren eingebetteten Satzes nicht relevant bezogen auf die Klärung von Q, da die Beantwortung von ?p = War Marche beim Friseur? zu keiner Reduktion des Alternativenraums von $\mathrm{Q}$ führt, es gilt demnach \# $(\Pi / \mathrm{A})=\mathrm{n}$. Folglich gehört der Komplementsatz zur Nebenstruktur und die Äußerung ist wohlgeformt.

Q: Warum ist Marche beleidigt?

B: Homer ignoriert, dass sie beim Friseur war.

Wird der Kontext in Form von Q dagegen so manipuliert, dass der Inhalt des Komplementsatzes [+at-issue]-Inhalt ausdrückt, während der Hauptsatz zur Nebenstruktur gehört, resultiert das in einer nicht-wohlgeformten Äußerung:

Q: Wo ist Marche?

B: \#Homer ignoriert, dass sie beim Friseur ist.

Dies gilt auch für andere Berücksichtigungsverben, wie beispielsweise vernachlässigen oder beachten. In Beispiel (57) drückt in den Äußerungen B und B' jeweils der Komplementsatz ohne seinen Bezugssatz die bezogen auf Q relevante Information aus, weswegen die Äußerungen in diesem Kontext nicht akzeptabel sind.

Q: Wie ist denn das Wetter?

B: \#Marche vernachlässigt, dass es regnet, und ist ohne Schirm aus dem

\footnotetext{
${ }^{16}$ Simons $(2007,1035)$ führt dieses Beispiel allerdings als Beleg dafür an, dass auch faktive Verben einen Nebensatz, der die Hauptaussage enthält, selegieren können. Dass es sich bei dem Verb to discover jedoch nicht um ein faktives, sondern um ein semifaktives Verb handelt, beweist das folgende Beispiel aus Hooper/Thompson (1973, 480). Wie für semifaktive Verben charakteristisch, wird die Faktivität aufgehoben, wenn der Satz in ein Konditionalsatzgefüge eingebettet ist (vgl. auch Beispiel (43) und Karttunen 1971).
}

(i) If I discover later that I have not told the truth, I will confess it to everyone. 
Haus gegangen.

B':\#Marche beachtet, dass es regnet, und hat sich einen Schirm eingepackt.

Die Beispiele zeigen, dass Komplementsätze von Berücksichtigungsverben nicht dazu geeignet sind, ohne ihren Bezugssatz [+at-issue]-Inhalt auszudrücken. Dasselbe gilt auch für die Komplemente von emotiven Verben. Ein Verb wie bereuen kann problemlos einen Satz einbetten, der für die Beantwortung der aktuellen Q nicht relevant ist:

Q: Warum haben sich Peter und Maria wieder versöhnt?

B: Sie bereut, dass sie ihn betrogen hat.

Drückt das sententiale Komplement eines emotiven Verbs dagegen [+at-issue]-Inhalt aus, ist die Äußerung nicht wohlgeformt, wie die Sätze B und B' in dem folgenden Beispiel zeigen:

Q: Wo ist denn Homer?

B: \#Marche bereut, dass sie ihn in den Kwik-E-Markt geschickt hat.

B':\#Marche nimmt ihm übel, dass er nach Las Vegas gefahren ist.

Es scheint, als würden die faktiven Verben der Gruppen iv. und v. nur Sätze selegieren, die nicht dazu geeignet sind, ohne ihren Bezugssatz eine quaestiobezogen relevante Information auszudrücken. Wird eine Lesart, in der der Nebensatz [+atissue]-Inhalt ausdrückt, erzwungen, resultiert dies in einem Akzeptabilitätsverlust der Äußerung.

Allerdings existieren auch Fälle, die auf den ersten Blick dieser Beobachtung zu widersprechen scheinen. In den beiden folgenden Beispielen handelt es sich um faktive Komplementsätze, welche die bezogen auf Q relevante Information ausdrücken, dennoch sind die Äußerungen wohlgeformt:

Q: Was ignoriert Homer?

B: (Er ignoriert,) dass Marche beim Friseur war.

Q: Was bereut Marche?

B': (Sie bereut,) dass sie Homer in den Kwik-E-Markt geschickt hat.

Muss die Aussage, dass faktive Komplementsätze ohne ihren Bezugssatz nicht zum Ausdruck von [+at-issue]-Inhalt geeignet sind, revidiert werden? Das muss sie nicht. In den beiden Beispielen oben drückt der jeweilige Nebensatz eindeutig eine bezogen auf Q relevante Information aus - entscheidend ist aber, dass der Nebensatz nur als Konstituente seines Matrixsatzes und nicht alleine Teil der Hauptstruktur ist. Der Matrixsatz kann in der jeweiligen Antwort in den Beispielen oben zwar weggelassen werden. In diesem Fall handelt es sich jedoch um elliptische Strukturen, die dadurch lizenziert sind, dass der ellidierte Matrixsatz bereits in der Frage enthalten ist. Folglich sind die Nebensätze in den Beispielen oben nicht alleine Träger der [+at-issue]Information, sondern nur als Konstituenten zusammen mit ihrem Bezugssatz, welcher weggelassen werden kann. Da in diesem Kapitel jedoch gerade getestet werden 
soll, welche Nebensätze ohne ihren Bezugssatz Träger von [+at-issue]-Information sein können (vgl. dazu Abschnitt den letzten Absatz aus Abschnitt 2.2.1), bilden die Beispiele (60) und (61) keine Gegenbeispiele zu der weiter oben gemachten Beobachtung, dass faktive Komplementsätze nie alleine [+at-issue] sein können. Dafür spricht auch, dass faktive Komplementsätze stets zentrale Eigenschaften von [-atissue]-Inhalten aufweisen, worauf in Abschnitt 3 genauer eingegangen wird. Dass die Komplementsätze in den Beispielen (60) und (61) nicht alleine [+at-issue] sind, lässt sich auch daran zeigen, dass die entsprechenden Entscheidungsfragen $? \mathrm{p}=$ War Marche beim Friseur? bzw. Hat Marche Homer in den Kwik-E-Markt geschickt? nicht zur Klärung der jeweiligen Frage Q beitragen.

Zusammenfassend können wir festhalten, dass es für die diskurssemantische Funktion eines Komplementsatzes entscheidend ist, durch welches Verb er eingebettet ist. Handelt es sich um ein Brückenverb der Klasse i. oder ii., so kann der Nebensatz je nach Kontext auch ohne seinen Bezugssatz [+at-issue]-Inhalt ausdrücken. Dasselbe gilt für Nebensätze, die die Argumentforderung eines semifaktiven Verbs erfüllen. Faktive Komplementsätze sind dagegen nicht dazu geeignet, ohne ihren Bezugssatz eine relevante Information bezogen auf Q auszudrücken und sind folglich gemäß der Definition (25) immer [-at-issue] (wobei sie natürlich zusammen mit ihrem Bezugssatz zur Hauptstruktur gehören können). Ob Komplementsätze, die Teil der Nebenstruktur sind, eine N-UDQ beantworten, wie u.a. Beaver (2012), Holler (2005) und Onea (2013) bezogen auf appositive Relativsätze argumentieren (vgl. dazu Abschnitt 2.1.2), muss an dieser Stelle offen bleiben.

\subsubsection{Adverbialsätze}

Betrachten wir nun im Folgenden Adverbialsätze im Hinblick auf die Frage, ob sie ohne ihren Bezugssatz [+at-issue]-Inhalte ausdrücken können oder nicht. Beginnen wir mit Temporalsätzen. Ist die in einem Temporalsatz ausgedrückte Proposition gemäß der Definition in (25) nicht relevant in Bezug auf die aktuelle Q, ist die Äußerung wohlgeformt, wie das folgende Beispiel belegt. Wenn in dem folgenden Beispiel p der im Temporalsatz ausgedrückten Proposition entspricht, dann lautet $? \mathrm{p}=$ Ist Peter in den Urlaub gefahren?. Die Beantwortung dieser Frage durch Ausschluss der Menge an Welten, in denen p bzw. $\neg$ p gilt, führt zu keiner Reduktion der durch Q eröffneten Bipartition \{Peter hat das Auto gekauft, Peter hat das Auto nicht gekauft $\}$. Es gilt demnach $\#(\Pi / A)=n$ und nach Definition (26) gehört der Temporalsatz somit zur Nebenstruktur.

Q: Hat Peter das Auto gekauft?

B: Ja, er hat es gekauft, bevor er in den Urlaub gefahren ist.

Wie zu erwarten, ist die Verwendung eines Temporalsatzes wohlgeformt, wenn er Teil der Nebenstruktur ist. In Beispiel (33), hier wiederholt als (63), haben wir bereits gesehen, dass Temporalsätze dagegen keinen [+at-issue]-Inhalt ausdrücken können. In dem folgenden Beispiel ist die aus dem Temporalsatz abgeleitete Frage 
$? \mathrm{p}=$ War Peter im Kino? fast identisch mit Q. Demnach ist die Beantwortung von ?p auch höchst relevant für die Beantwortung von $\mathrm{Q}$, da eine vollständige Antwort auf ?p auch einer kompletten Beantwortung von Q entspricht. Wie in Abschnitt 2.1 bereits gezeigt, ist die Äußerung nicht wohlgeformt.

Q: War Peter gestern im Kino?

B: \#Peter hat seine Mutter besucht, nachdem er im Kino war.

Daraus können wir schließen, dass ein mit nachdem eingeleiteter Adverbialsatz nicht Teil der Hauptstruktur sein kann. Dasselbe gilt auch für durch andere Subjunktionen eingeleitete Temporalsätze. Wird durch den Kontext eine Lesart erzwungen, in der die im Temporalsatz ausgedrückte Proposition im Sinne der Definition (25) bezogen auf Q relevant ist, ist die Verwendung eines Temporalsatzes nicht angemessen.

Q: Hat Peter die Klausur mitgeschrieben?

B: \#Peter war müde, als er die Klausur geschrieben hat.

B':\#Peter war richtig schlecht gelaunt, bevor er die Klausur geschrieben hat.

Wie bereits in Fußnote (8) in Abschnitt 2.1 erwähnt, darf diese Verwendung nicht mit einer Äußerung, in der Q bereits durch $J a$ oder eine andere Antwortpartikel beantwortet wurde, verwechselt werden. In dem folgenden Beispiel wird Q zum Zeitpunkt $\mathrm{t}_{1}$ mit $J a$ beantwortet, indem eine Aufforderung ergeht, alle Welten, in denen Peter die Klausur nicht mitgeschrieben hat, zu entfernen.

Q: Hat Peter die Klausur mitgeschrieben?

B: Ja, Peter war richtig schlecht gelaunt, bevor er die Klausur geschrieben hat.

Die Äußerung $J a$ liefert demnach eine vollständige Antwort, indem sie zwischen den beiden Alternativen $\mathrm{p}$ und $\neg \mathrm{p}$ die Proposition $\mathrm{p}$ auswählt. Der Temporalsatz wird dagegen zu einem späteren Zeitpunkt $t_{2}$ geäußert. Zu diesem Zeitpunkt ist Q bereits ausreichend geklärt. Entscheidend ist nun, dass nach Roberts (1996) eine Frage Q nur solange relevant und aktuelles Diskurstopik ist, bis sie komplett beantwortet oder aber als unbeantwortbar entlarvt wurde (vgl. Abschnitt 2.1). Dies bedeutet, dass Q zum Zeitpunkt $t_{2}$ nicht mehr der Quaestio entspricht. Der Äußerung Peter war richtig schlecht gelaunt, bevor er die Klausur geschrieben hat. liegt vielmehr bereits eine neue Frage Q' zugrunde, beispielsweise die Frage nach dem Gemütszustand Peters vor der Klausur. Bezogen auf diese Frage ist der Inhalt des Temporalsatzes gemäß der Definition (25) nicht relevant. Demnach ist der Temporalsatz zum Äußerungszeitpunkt nicht Teil der Haupt-, sondern der Nebenstruktur und insofern ist es nicht weiter verwunderlich, dass die Äußerung absolut wohlgeformt ist.

Bereits im vorherigen Abschnitt wurde gezeigt, dass faktive Komplementsätze, die eigentlich nicht zum Ausdruck von [+at-issue]-Inhalt geeignet sind, die Antwort auf eine Konstituentenfrage enthalten können (vgl. die Beispiele (60) und (61)). Ganz analog dazu existieren auch Fälle, in denen ein Temporalsatz die für die Beantwortung einer Frage relevante Information enthält, und zwar dann, wenn das Fragewort 
ein Adverbial ist wie in dem folgenden Beispiel. In diesem Fall kann dann auch der Matrixsatz in der Antwort ellidiert werden.

Q: Wann hat Peter das Auto gekauft?

B: (Er hat es gekauft,) bevor er umgezogen ist.

Genau wie bezogen auf die Beispiele (60) und (61) kann hier argumentiert werden, dass der Temporalsatz, selbst wenn der Bezugssatz ellidiert wird, nicht alleiniger Träger der [+at-issue]-Information ist, sondern nur als Konstituente seines Matrixsatzes zusammen mit diesem zur Hauptstruktur gehört (vgl. dazu auch den letzten Absatz von Abschnitt 2.2.1 sowie die Diskussion der Beispiele (60) und (61)). Dies zeigt sich auch daran, dass der Temporalsatz gemäß der Definition in (25) nach Simons et al. (2011) [-at-issue]-Inhalt ausdrückt. Wichtig hierbei ist, dass zur Ermittlung der Frage ?p die nebensatzeinleitende Subjunktion gestrichen werden muss (vgl. (36) und Simons et al. 2011). Dann gilt bezogen auf Beispiel (66):

$$
\begin{aligned}
& \mathrm{p}=\text { Peter ist umgezogen } \\
& ? \mathrm{p}=\text { Ist Peter umgezogen? }
\end{aligned}
$$

Entscheidend ist nun, dass die Klärung der Entscheidungsfrage ?p zu keiner Reduktion der durch Q eröffneten Alternativenmenge führt. Werden alle Welten, in denen Peter umgezogen bzw. nicht umgezogen ist, aus dem jeweiligen Modell entfernt, bleibt die durch $\mathrm{Q}$ eröffnete Alternativenmenge davon unberührt, es gilt folglich $\#(\Pi / \mathrm{A})=\mathrm{n}$. Demnach gehört der Temporalsatz entgegen unserer ersten Intuition nicht zur Haupt-, sondern zur Nebenstruktur. [+At-issue] ist nur die Gesamtäußerung B, wobei der Temporalsatz eine Konstituente des Satzes bildet, nicht aber die im Temporalsatz enthaltene Proposition für sich alleine. Aus diesem Grund ist Beispiel (66), anders als (63), wo der Temporalsatz tatsächlich [+at-issue] ist, wohlgeformt.

Auch das folgende Beispiel wirkt auf den ersten Blick wie ein Gegenbeispiel. Hier drückt der Temporalsatz eindeutig [+at-issue]-Inhalt aus, da die Beantwortung der Frage $? \mathrm{p}=$ Fiel Peters Chef vom Stuhl? auch zur Klärung von Q führt.

Q: Was ist passiert, dass Peter so gelacht hat?

B: Peter musste lachen, als sein Chef vom Stuhl fiel.

Jedoch handelt es sich bei dem Adverbialsatz zwar um einen durch eine temporale Subjunktion eingeleiteten Satz, die Relation zwischen Haupt- und Nebensatz wird allerdings kausal interpretiert. In diesem Fall scheint die Verwendung als Teil der Hauptstruktur möglich zu sein. Ist dagegen eine kausale Anreicherung der temporalen Relation nicht naheliegend, ist eine derartige Verwendung stark markiert. Die beiden Äußerungen B und B' in Beispiel (69) drücken die gleiche temporale Relation aus. Als Antwort auf die aktuelle Frage Q bildet aber nur B' eine wohlgeformte Äußerung, da die bezogen auf Q relevante Information in dem Fall im Haupt- und nicht im Temporalsatz ausgedrückt wird. 
Q: Was ist passiert, nachdem Peter in den Urlaub gefahren ist?

B: ?Peter ist in den Urlaub gefahren, bevor in Hamburg EHEC ausgebrochen ist.

B': In Hamburg ist EHEC ausgebrochen, nachdem Peter in den Urlaub gefahren ist.

Wir können also festhalten, dass Temporalsätze immer Teil der Nebenstruktur sind. Wie bei faktiven Komplementsätzen scheint es sich dabei auch nicht um eine kontextuell streichbare Eigenschaft zu handeln, da die Verwendung eines Temporalsatzes in einem Kontext, in dem sein Inhalt relevant für die aktuelle Q ist, nicht wohlgeformt ist. Da Temporalsätze keine kontextuelle Umdeutung als Träger von [+at-issue]Inhalten zulassen, schließe ich, dass temporale Subjunktionen konventionelle Markierungen für die Zugehörigkeit zur Nebenstruktur sind. ${ }^{17}$ Ausnahmen bilden kausal interpretierte Temporalsätze, welche auch als Teil der Hauptstruktur verwendet werden können. Aus diesem Grund ist es naheliegend, dass auch Kausalsätze [+atissue]-Inhalte ausdrücken können, was im Folgenden untersucht werden soll. Dabei werde ich mich auf Kausalsätze konzentrieren, die mit weil eingeleitet werden. ${ }^{18}$ Betrachten wir zunächst in Beispiel (70) die Verwendung eines Kausalsatzes in einem Kontext, in dem der Kausalsatz gemäß der Definition (25) nicht zur Hauptstruktur gehört. Wenn $\mathrm{p}=$ Peter hat zu wenig gelernt, dann ist die Beantwortung von $? \mathrm{p}=$ Hat Peter zu wenig gelernt? nicht hilfreich für die Klärung der aktuellen Frage Q. Q wird vielmehr durch den dem Kausalsatz vorhergehenden Hauptsatz beantwortet, wohingegen der Kausalsatz in Form einer Erklärung Teil der Nebenstruktur ist. Wie wir sehen, ist eine solche Verwendung absolut wohlgeformt.

Q: Warum ist Peter so traurig?

B: Er ist durchgefallen, weil er zu wenig gelernt hat.

${ }^{17}$ Anders als kanonische Temporalsätze, die nicht dazu geeignet sind, ohne ihren Bezugssatz [+atissue]-Informationen auszudrücken, können periphere als-Sätze auch alleine Teil der Hauptstruktur sein. So beobachten Zifonun et al. (1997, 2325f) anhand des folgenden Beispiels: „Gegenüber der üblichen Verteilung von Haupt- und Nebeninformation jeweils auf Haupt- und Nebensatz liegt also eine Umkehr vor."

(i) Ich stand gerade an der Haustür, als auch schon/plötzlich das Gewitter einsetzte.

Dazu passt, dass die Äußerung als Antwort auf die Frage Hat es ein Gewitter gegeben? wohlgeformt ist. Periphere Temporalsätze bilden demnach eine Ausnahme zu der oben formulierten Regel, derzufolge Temporalsätze nie ohne ihren Bezugssatz Teil der Hauptstruktur sein können. Allerdings handelt es sich bei peripheren Temporalsätzen um keine gewöhnlichen Temporalsätze. Zum einen werden sie nach Zifonun et al. (1997, 2325f) immer mit als eingeleitet und treten stets nachgestellt auf. Zudem unterscheidet sich ihr semantischer Beitrag von dem kanonischer Temporalsätze, genauer gesagt handelt es sich nach Zifonun et al. (1997, 2325) um Temporalsätze „die nicht in der üblichen Weise als Spezifikatoren der Betrachtzeit für den Obersatz interpretiert werden können“ (zur Semantik von Temporalsätzen vgl. auch Teil III). Es ist also nicht verwunderlich, dass sich periphere Temporalsätze diskurssemantisch anders verhalten als kanonische Temporalsätze, wie sie in diesem Abschnitt beschrieben wurden.

${ }^{18} \mathrm{Zu}$ da-Sätzen liegt mit Averintseva-Klisch/Volodina (2012) eine Studie vor, die zu dem Schluss kommt, dass $d a$-Sätze grundsätzlich keinen [+at-issue]-Inhalt ausdrücken können. 
Anders als Temporalsätze können weil-Sätze aber auch in Kontexten verwendet werden, in denen der Kausalsatz eine zur Beantwortung von Q relevante Information enthält. Dies ist in dem folgenden Beispiel illustriert (vgl. auch Holler 2009, die ebenfalls argumentiert, dass ein weil-Satz die kommunikativ relevante Information ausdrücken kann).

Kontext: B fängt im Büro plötzlich an zu lachen.

Q: Was ist denn los?

B: Ach, ich musste nur so lachen, weil Peter vom Stuhl gefallen ist.

In diesem Beispiel führt die Klärung der Frage ?p = Ist Peter vom Stuhl gefallen? zu einer Reduktion der durch Q eröffneten Alternativenmenge bis auf ein Element. Es gilt demnach $\#(\Pi / \mathrm{A})=1$ und folglich drückt die im Kausalsatz ausgedrückte Proposition [+at-issue]-Inhalt aus.

Häufiger ist zudem die Verwendung eines weil-Satzes, der eine implizite, das heißt nicht explizit formulierte Q beantwortet. Betrachten wir dazu das folgende Beispiel.

Q: Hat Peter gestern die Klausur mitgeschrieben?

B: Nein, er konnte sie nicht mitschreiben, weil er krank ist.

Wie in Beispiel (62) wird Q durch eine Partikel beantwortet und ist demnach zum Zeitpunkt der Äußerung des Nebensatzes nicht mehr aktuelles Diskurstopik. Allerdings wirft die Beantwortung von Q durch Nein eine weitere, implizite Frage Q' auf, nämlich warum Peter die Klausur nicht geschrieben hat. Bezeichnen wir Q' als Warum q?. Q' wird nun durch den Kausalsatz beantwortet, da die Beantwortung von ?p = Ist Peter krank? eine Antwort auf die Frage Q' = Warum hat er die Klausur nicht mitgeschrieben? impliziert. Die Klärung von ?p ist also relevant für die Klärung von Warum q? (=Q'), demnach ist der Kausalsatz in Beispiel (72) Teil der Hauptstruktur von Q' und das Beispiel ein Beleg dafür, dass Kausalsätze zum Ausdruck von [+at-issue]-Inhalten geeignet sind.

Worin besteht nun aber der Unterschied zu der Verwendung von temporalen Adverbialsätzen in einem derartigen Kontext? Betrachten wir dazu noch einmal Beispiel (62), hier wiederholt als (73).

Q: Hat Peter das Auto gekauft?

B: Ja, er hat es gekauft, bevor er in den Urlaub gefahren ist.

Nehmen wir einmal analog zu Beispiel (72) an, dass durch die Beantwortung von Q durch die Partikel $J a$ eine weitere, implizite Frage Q' aufgeworfen wird, die potentiell durch den Temporalsatz beantwortet werden könnte. Dann müsste Q' $=$ Wann hat Peter das Auto gekauft?, also Wann q? lauten. Entscheidend ist nun, dass der Temporalsatz gemäß der Definition (25) nach Simons et al. $(2011,8)$ keine Antwort auf Q' liefert, da die Frage ?p = Ist Peter in den Urlaub gefahren? nicht relevant bezogen auf Q' ist. Wichtig ist dabei, dass nebensatzeinleitende Subjunktionen nicht Teil der ausgedrückten Proposition $\mathrm{p}$ und demnach auch nicht der entsprechenden 
Entscheidungsfrage ?p sind (vgl. (36)). Da die Klärung von ?p nicht relevant für die Klärung von $Q^{\prime}=$ Wann $q$ ? ist, gehört der Temporalsatz in Beispiel (73) zur Nebenstruktur, auch wenn wir davon ausgehen, dass eine weitere implizite Frage aufgeworfen wird.

Wir können demnach festhalten, dass ein grundlegender Unterschied zwischen Temporal- und weil-Sätzen besteht. Die in einem Kausalsatz ausgedrückte Proposition p kann gemäß der Definition (25) eine Antwort auf die entsprechende Frage Q ( Warum q?) liefern. Dagegen sind Temporalsätze nicht in der Lage eine entsprechende implizite oder explizite Frage $\mathrm{Q}=$ Wann q? zu beantworten. Während also eine Proposition $\mathrm{p}=$ Peter ist krank auch ohne kausale Subjunktion eine Begründung für etwas ausdrücken kann, kann dieselbe Proposition ohne Einleitung durch eine temporale Subjunktion wie bevor oder nachdem kein Zeitintervall zur Beantwortung der Frage Wann q? liefern. Die Rekonstruktion einer temporalen Relation zwischen den beiden Sachverhalten ist hier nicht möglich. In Teil III werde ich anlehnend an Johnston (1994) dafür argumentieren, dass es einen grundlegenden Unterschied zwischen temporalen und kausalen Subjunktionen gibt, der sich an dieser Stelle bereits zeigt: Während kausale Subjunktionen zweistellige Ausdrücke sind, die zwei Ereignisse miteinander verknüpfen, sind temporale Subjunktionen einstellig und bilden ihr Argument, eine Proposition, auf ein Intervall i ab. Zur Beantwortung einer Frage Wann? ist nun aber eine Antwort des Typs i erforderlich, also die Nennung eines Zeitintervalls. Da die durch eine temporale Subjunktion eingeleitete Proposition ohne den Satzeinleiter kein solches Intervall ausdrückt, kann ein Temporalsatz in einem Beispiel wie (73) auch nicht als Antwort auf eine implizite Frage Wann? fungieren (für mehr Details vgl. Teil III). ${ }^{19}$

\subsubsection{Relativsätze}

Betrachten wir zum Abschluss noch abhängige Sätze, die von einem nominalen Antezedens abhängen. Bezogen auf die Frage, ob Relativsätze ohne ihren Bezugssatz [+at-issue]-Inhalt ausdrücken können, ist es wichtig, zwischen restriktiven und nichtrestriktiven Relativsätzen zu unterscheiden. Die beiden Nebensatztypen divergieren in zahlreichen prosodischen, syntaktischen, semantischen und illokutions- bzw. informationsstrukturellen Gesichtspunkten, welche ausführlich in Holler (2005, 25ff) diskutiert werden. Etwas vereinfacht kommt Holler (2005) dabei zu dem Schluss, dass

${ }^{19}$ Der in diesem Abschnitt beschriebene diskurssemantische Unterschied zwischen Temporal- und weil-Sätzen zeigt sich meiner Meinung nach auch in dem folgenden Beispiel. Aufgrund der Frage Q kann davon ausgegangen werden, dass dem Fragenden zum Sprechzeitpunkt nicht bereits bekannt ist, dass das entsprechende Schiff unterging - der Nebensatzinhalt ist somit relevant bezogen auf Q. In einem solchen Kontext bildet aber nur B', nicht B, eine wohlgeformte Antwort auf Q.

(i) Q: Was ist denn los?

B: \#Der Kapitän sendete einen Notruf, bevor das Schiff unterging.

B': Der Kapitän sendete einen Notruf, weil das Schiff unterging. 
die in der Literatur genannten phono-syntaktischen Kriterien für die Unterscheidung zwischen restriktiven und appositiven Relativsätzen nur bedingt, semantische und illokutions- bzw. informationsstrukturelle Kriterien dagegen uneingeschränkt verwendbar sind. Ein wichtiges Unterscheidungskriterium ist die Tatsache, dass restriktive Relativsätze immer einen Beitrag zur Bestimmung des durch sie modifizierten Antezedens leisten, wohingegen nicht-restriktive Relativsätze einen bereits identifizierten Diskursreferenten näher beschreiben und demnach, ohne die Fixierung des Diskursreferenten zu beeinträchtigen, weglassbar sind (für eine genauere Darstellung der einzelnen Unterscheidungskriterien vgl. Holler 2005, 25ff). Im Folgenden werden zuerst nicht-restriktive und anschließend restriktive Relativsätze daraufhin untersucht, ob sie ohne ihren Bezugssatz die aktuelle QUD beantworten können.

Nicht-restriktive Relativsätze bilden keine homogene Klasse, so zeigt Holler (2005), dass nicht-restriktive Relativsätze mit dem Diskurs entweder symmetrisch, dann liegt ein weiterführender Relativsatz vor, oder asymmetrisch verknüpft werden können, was auf appositive Relativsätze zutrifft. In der vorliegenden Arbeit werden lediglich appositive Relativsätze betrachtet. ${ }^{20}$

Gehört ein appositiver Relativsatz zur Nebenstruktur, ist die Gesamtäußerung wohlgeformt wie in dem folgenden Beispiel. In Abschnitt 2.1.2 wurde jedoch bereits darauf hingewiesen, dass ein appositiver Relativsatz wie in Beispiel (74) nach u.a. Beaver (2012) und Onea (2013) dazu dient, eine für den weiteren Diskursverlauf nicht relevante N-UDQ zu beantworten.

Q: Was hast du gestern gemacht?

B: Ich habe mit Jap telefoniert, der gerade in Indonesien ist.

Drückt der appositive Relativsatz dagegen [+at-issue]-Inhalt aus, während sein Bezugssatz zur Nebenstruktur gehört, ist die Äußerung nicht wohlgeformt, wie bereits Bartsch $(1978,8)$, Hartmann $(1984,313 f)$ und Holler (2009) beobachtet haben. In dem folgenden Beispiel führt die Klärung von ?p = Ist Jap gerade in Indonesien? zu einer Reduktion der durch Q eröffneten Alternativenmenge, der Relativsatz gehört demnach zur Hauptstruktur, und das Beispiel ist nicht wohlgeformt.

Q: Wo ist eigentlich Jap im Moment? Ich habe ihn schon lange nicht mehr gesehen.

B: \#Jap, der gerade in Indonesien ist, verreist nicht gern.

Dass appositive Relativsätze nicht ohne ihren Bezugssatz [+at-issue]-Inhalt ausdrücken können, zeigt auch das folgende Beispiel.

Q: Was ist passiert, dass Peter so gelacht hat?

B: ?Peters Chef, der gerade vom Stuhl gefallen ist, wohnt um die Ecke.

\footnotetext{
${ }^{20}$ Bezogen auf die diskurssemantische Funktion von weiterführenden Relativsätzen zeigt Holler (2005, 213ff), dass sich diese auf eine eigene, zumeist implizite Quaestio beziehen (vgl. dazu auch Brandt 1990).
} 


\section{Zur Interpretation von Sätzen im Kontext}

Hier enthält der Relativsatz die Information, welche typischerweise eine passende Antwort auf Q darstellt. Denn wenn wir über keine weiteren Kontextinformationen verfügen, ist anzunehmen, dass die im Relativsatz geschilderte Situation eher Auslöser eines Lachanfalls ist als Informationen über den Wohnsitz des Chefs. Dennoch wird B's Äußerung so interpretiert, dass der Hauptsatz Q beantwortet. Dass appositive Relastivsätze auch im Englischen nicht zum Ausdruck von [+at-issue]Inhalt geeignet sind, zeigt Beaver (2012) anhand des folgenden Kurzdialogs:

Q: Where's Bob these days?

B: \#Bob, who is in Austin, hasn't called me for a week

Wir können festhalten, dass appositive Relativsätze nicht dazu geeignet sind, ohne ihren Bezugsatz [+at-issue]-Inhalt auszudrücken.

Wie verhalten sich nun restriktive Relativsätze? Bei der Betrachtung von restriktiven Relativsätzen spielen Definitheit und Spezifizität eine wichtige Rolle. Restriktive Relativsätze, die ein definites Antezedens modifizieren, verhalten sich bezogen auf ihre diskurssemantische Funktion wie appositive Relativsätze. So ist die Äußerung eines restriktiven Relativsatzes, der sich auf eine definite NP bezieht, nur dann wohlgeformt, wenn der Relativsatz keine Beantwortung der aktuellen Q impliziert wie in dem folgenden Beispiel.

Q: Warum ist Peter so gut gelaunt?

B: Er hat die Klausur bestanden, die er in Strafrecht geschrieben hat.

Ein Beispiel, für einen restriktiven Relativsatz mit einem definiten Antezedens, der [+at-issue]-Inhalt ausdrückt, bildet (79). Hier ist eindeutig der Relativsatz und nicht sein Bezugssatz relevant bezogen auf Q und, wie wir feststellen können, ist die Äußerung in dem entsprechenden Kontext zumindest sehr stark markiert. So verrät die aktuelle Q, dass der Fragende keine Kenntnis über die Aufnahme der Inszenierung durch die Kritiker besitzt. Aus diesem Grund ist die Verwendung der definiten NP der Kritiker in Zusammenhang mit dem [+at-issue]-Relativsatz nicht angemessen, da diese präsupponiert, dass der entsprechende Diskursreferent bereits bekannt ist.

Q: Wie wurde die Inszenierung von der Presse beurteilt?

B: ?Maria trifft gerade den Kritiker, der eine sehr gute Besprechung abgegeben hat.

Auch in dem folgenden Beispiel ist der Inhalt des restriktiven Relativsatzes relevant bezogen auf Q.

(80) Hintergrund: Ein in die Tage gekommener Linguist wird von einem Journalisten interviewt.

Q: Während Ihrer Laufbahn als Linguist, was hat Sie am meisten beeindruckt?

B: Ich kenne den Linguisten, der nicht lesen kann. 
Obwohl der Inhalt des Relativsatzes eine plausiblere Antwort auf die Frage darstellt, wird B's Äußerung so interpretiert, dass der Hauptsatz die Hauptaussage darstellt. Was den Befragten also am meisten beeindruckt hat, ist nicht die Tatsache, dass es einen Linguisten gibt, der nicht lesen kann, sondern vielmehr die Tatsache, dass er diesen (als bekannt vorausgesetzten) analphabetischen Linguisten kennt. Dies bedeutet, dass der Relativsatz als Teil der Nebenstruktur interpretiert wird, obwohl die andere Lesart plausibler wäre. Wir können also festhalten, dass restriktive Relativsätze mit einem definiten Antezedens nicht dazu geeignet sind, ohne ihren Bezugssatz die aktuelle Q zu beantworten.

Bei der Betrachtung von restriktiven Relativsätzen mit einem indefiniten Antezedens ist das Konzept der Spezifizität von entscheidender Bedeutung. Indefinite NPn können danach unterteilt werden, ob sie sich auf einen spezifischen oder einen nichtspezifischen Diskursreferenten beziehen. Zentral ist dabei das Wissen von S und H um den jeweiligen Diskursreferenten. Während eine spezifische indefinite NP signalisiert, dass der Diskursreferent $\mathrm{S}$ aber nicht $\mathrm{H}$ bekannt ist, zeigen nicht-spezifische NPn an, dass der entsprechende Diskursreferent beiden Gesprächsteilnehmern unbekannt ist. Spezifische NPn referieren somit auf eine bestimmte, nicht-spezifische auf eine nicht näher bestimmbare Entität (vgl. zum Konzept der Spezifizität u.a. Farkas 2002, Fodor/Sag 1982, von Heusinger 2002 und von Heusinger 2011). Da Deutsch über keine overte Markierung für Spezifizität verfügt, sind indefinite Ausdrücke im Deutschen ambig, da sie entweder spezifisch oder nicht-spezifisch interpretiert werden können. Häufig kann auf die intendierte Lesart erst durch den folgenden Kontext geschlossen werden. Beispielsweise wird die indefinite NP eine Freundin in dem folgenden Diskurs erst durch die Aufnahme durch die unterschiedlichen Pronominalausdrücke disambiguiert.

(81) Peter wollte mit einer Freundin ins Kino gehen.

a. Aber er konnte keine erreichen. (nicht-spezifisch)

b. Aber er konnte sie nicht erreichen. (spezifisch)

Bezieht sich ein restriktiver Relativsatz nun auf einen nicht-spezifischen Diskursreferenten, kann er nicht ohne seinen Bezugssatz [+at-issue]-Inhalt ausdrücken. Aus diesem Grund ist B's Äußerung in dem folgenden Kontext nicht wohlgeformt:

Q: Kann das jemand heilen?

B: ?Ich suche einen Arzt, der das heilen kann.

Handelt es sich dagegen um eine spezifisch interpretierte NP, kann ein restriktiver Relativsatz dazu verwendet werden, die aktuelle $\mathrm{Q}$ ohne seinen Bezugssatz zu beantworten, wie die folgenden beiden Beispiele zeigen.

(83) Q: Wie wurde die Inszenierung von der Presse beurteilt?

B: Maria trifft gerade einen Kritiker, der eine sehr gute Besprechung abgegeben hat.

Q: Gibt es eigentlich auch bunte Fische? 
B: Maria hat einen Fisch gefangen, der rot-blau gestreift ist.

Natürlich können restriktive Relativsätze mit einem indefiniten Antezedens auch Teil der Nebenstruktur sein:

Q: Was macht Peter in der Buchhandlung?

B: Er sucht ein Buch, das ihm eine Freundin empfohlen hat.

Die Tatsache, ob ein restriktiver Relativsatz zum Ausdruck von [+at-issue]-Inhalt geeignet ist, während sein Bezugssatz [-at-issue]-Inhalt enthält, hängt demnach von der Art seines Antezedens ab: Ist dieses definit, ist der Relativsatz für die Beantwortung der aktuellen Q immer irrelevant. Handelt es sich dagegen um ein indefinites Antezedens, kann der Nebensatz auch für die Beantwortung von Q relevante Informationen enthalten, wenn es sich um einen spezifischen Diskursreferenten handelt.

\subsubsection{Zusammenfassung}

Die Diskussion hat gezeigt, dass sich die im Rahmen dieser Arbeit diskutierten Nebensatztypen bezogen auf ihre diskurssemantische Funktion stark voneinander unterscheiden. Die relevanten Ergebnisse sind in Tabelle 2.1 noch einmal zusammengefasst.

\begin{tabular}{|l|c|c|}
\hline Nebensatztyp & {$[$-at-issue $]$} & {$[+$ at-issue $]$} \\
\hline \hline Komplementsatz, Brückenverb & + & + \\
\hline Semifaktiver Komplementsatz & + & + \\
\hline Faktiver Komplementsatz & + & - \\
\hline Temporaler Adverbialsatz & + & - \\
\hline Adverbialsatz mit weil & + & + \\
\hline Appositiver Relativsatz & + & - \\
\hline Restriktiver Relativsatz, definites Antezedens & + & - \\
\hline $\begin{array}{l}\text { Restriktiver Relativsatz, indefinites Antezedens, } \\
\text { nicht-spezifisch }\end{array}$ & + & + \\
\hline $\begin{array}{l}\text { Restriktiver Relativsatz, indefinites Antezedens, } \\
\text { spezifisch }\end{array}$ & & - \\
\hline
\end{tabular}

Tabelle 2.1.: Nebensätze und die Beantwortung von Q

Die untersuchten Nebensatztypen können bezogen auf ihre diskurssemantische Funktion demnach in zwei Gruppen unterteilt werden:

\section{Gruppe 1:}

Der diskurssemantische Status dieser abhängigen Sätze hängt vom Kontext ab, da sie je nach Kontext entweder zur Haupt- oder zur Nebenstruktur gehören können. 
In diese Gruppe gehören u.a.: Komplementsätze von Brücken- und semifaktiven Verben, mit weil eingeleitete Kausalsätze, restriktive Relativsätze mit einem indefiniten spezifischen Antezedens.

\section{Gruppe 2:}

Der diskurssemantische Status von diesen abhängigen Sätzen ist nicht kontextabhängig, da diese Sätze ohne ihren Bezugssatz keine für die Beantwortung der aktuellen Q relevante Information ausdrücken können.

Zu Gruppe 2 zählen u.a.: Komplementsätze von faktiven Verben, Temporalsätze, appositive Relativsätze, restriktive Relativsätze mit einem definiten Antezedens, restriktive Relativsätze mit einem indefiniten nicht-spezifischen Antezedens.

Die Ergebnisse der Untersuchung bestätigen den Eindruck von Brandt $(1989,1996)$ und Holler (2009), dass die Unterscheidung zwischen Haupt- und Nebensätzen das kommunikative Gewicht der jeweiligen Äußerung nicht eindeutig festlegt. Auch ein Satz, der alle der für Nebensätze typischen Merkmale aufweist, kann Träger von [+at-issue]-Inhalt sein. Wir haben aber auch gesehen, dass die Sätze der Gruppe 2 in einem Kontext, in dem sie [+at-issue]-Inhalt ausdrücken, nicht wohlgeformt sind. Daraus ergibt sich die Frage, aus welchem Grund die jeweiligen Nebensatztypen nicht zur Hauptstruktur gehören können. Die Ursachen hierfür sind ganz unterschiedlich und sollen an dieser Stelle nur kurz angeschnitten werden. In Kapitel 4 werde ich dafür argumentieren, dass Präsuppositionen eine Untermenge der [-at-issue]-Inhalte bilden. Dass einige Nebensätze grundsätzlich keinen [+at-issue]-Inhalt ausdrücken können, ist dann darauf zurückzuführen, dass diese Sätze präsupponiert sind. Dies trifft vor allem auf faktive Komplementsätze, aber auch auf Temporalsätze zu, wie ich in Kapitel 4 und ausführlicher noch in Abschnitt 9.1 zeigen werde. Restriktive Relativsätze gehören nur dann konventionell zur Nebenstruktur, wenn sie ein definites Antezedens modifizieren. Definite NPn signalisieren nun aber konventionell die Zugehörigkeit zur Nebenstruktur, da die Verwendung eines definiten Artikels anzeigt, dass nicht die Existenz des jeweiligen Referenten zur Debatte steht, sondern dass vielmehr etwas über diesen vorausgesetzten Referenten ausgesagt werden soll (vgl. Onea 2011). Eine definite NP ist demnach immer [-at-issue], wohingegen das, was über sie ausgesagt wird, die Hauptstruktur einer Äußerung bildet. Modifiziert nun ein restriktiver Relativsatz eine definite NP, bildet er mit ihr zusammen eine syntaktische und semantische Einheit. Aus diesem Grund ist es nicht verwunderlich, dass restriktive Relativsätze mit einem definiten Antezedens ebenfalls immer zur Nebenstruktur gehören.

Die Tatsache, dass appositive Relativsätze immer [-at-issue]-Inhalt ausdrücken, muss dagegen anders begründet werden, da die nicht-restriktiven Relativsätze gerade nicht präsupponiert sind, worauf in den Kapiteln 3 und 4 noch genauer eingegangen wird. Dass sie dennoch nie zur Beantwortung der aktuell relevanten Q verwendet werden können, zeigt, dass die gesamte Satzkonstruktion konventionell die Zugehörigkeit zur Nebenstruktur markiert. Wie bereits Hartmann (1984, 313f) beobachtet, dienen appositive Relativsätze anders als restriktive nicht der Einschränkung eines Refe- 


\section{Zur Interpretation von Sätzen im Kontext}

renzbereichs und sind somit nicht notwendig für die Identifizierung des intendierten Referenten. Stattdessen besteht ihre Funktion in der informationsstrukturellen Abwertung von Inhalten, kurz: Die Verwendung eines appositiven Relativsatzes dient der Kennzeichnung von [-at-issue]-Inhalten. Dies korreliert mit einer spezifischen Form. Häufig werden appositive Relativsätze dadurch charakterisiert, dass sie Kommaintonation aufweisen, d.h. prosodisch von den sie umgebenden Äußerungsteilen abgegrenzt werden (vgl. beispielsweise Brandt 1990, Lehmann 1984, 263 und Zifonun et al. 1997, 2007f). Holler (2005) weist in ihrer Diskussion der wichtigsten Kriterien zur Unterscheidung zwischen restriktiven und appositiven Relativsätzen jedoch unter Rückbezug auf Batliner et al. (1995) darauf hin, dass dieses Unterscheidungskriterium bisher experimentell nicht belegt werden konnte. Als belegt gilt dagegen lediglich die Tatsache, dass nach einem appositiven Relativsatz eine intonatorische Pause gemacht wird (vgl. Mayer 1999) und dass appositive Relativsätze immer einen eigenen Akzent tragen, restriktive Relativsätze dagegen nicht zwangsläufig (vgl. für eine ausführlichere Darstellung Holler 2005, 25ff). Auch wenn appositive Relativsätze nicht zwangsläufig Kommaintonation aufweisen, unterscheiden sie sich formal somit dennoch eindeutig von restriktiven Relativsätzen, so dass die Annahme von konstruktionsspezifischen Selektionsbeschränkungen durchaus plausibel ist.

\subsection{Zusammenfassung des Kapitels}

In diesem Kapitel wurde das kommunikative Gewicht von ausgesuchten Nebensätzen untersucht. Implementiert wurde die Untersuchung in ein fragebasiertes Diskursmodell nach Klein/von Stutterheim (1992), Roberts (1996), Simons et al. (2011) und von Stutterheim (1989), deren zentrale Idee darin besteht, dass jedem Text eine explizite oder implizite Frage Q zugrunde liegt. Eine Proposition p ist dabei genau dann [+at-issue], wenn die Beantwortung der aus p abgeleiteten Entscheidungsfrage ?p zu einer Reduktion des durch Q eingeführten Alternativenraums führt. In der germanistischen Literatur zur Reliefgebung (vgl. u.a. Bartsch 1978, Hartmann 1984, Posner 1972, von Stutterheim 1989 und Weinrich 1964) wurde häufig postuliert, dem Unterschied zwischen Haupt- und Nebensatz entspreche die Differenz zwischen Haupt- und Nebeninformation. Diese Beobachtung konnte durch die Untersuchung ausgewählter Nebensatztypen nicht bestätigt werden, vielmehr zeigte sich, wie bereits von Brandt (1989), Brandt (1996) und Holler (2009) herausgearbeitet, dass syntaktische Subordination nicht eindeutig die kommunikative Irrelevanz des Geäußerten markiert. So hat sich herausgestellt, dass einige Nebensatztypen durchaus dazu verwendet werden können, die aktuell relevante Q zu beantworten, wohingegen andere Nebensatztypen eine solche Verwendung ausschließen. Da in bisherigen Studien lediglich einzelne Nebensatztypen daraufhin untersucht wurden, ob sie Träger der Hauptinformation sein können, wird mit der in diesem Kapitel durchgeführten systematischen Untersuchung von acht Nebensatztypen (drei Arten von Komplementsätzen, zwei verschiedene Adverbial- und drei verschiedene Relativsatztypen) eine wesentliche Lücke geschlossen. 


\section{Typische Eigenschaften der Nebenstruktur}

In Kapitel 2 wurde die Unterscheidung von [+at-issue]- und [-at-issue]-Inhalten vorgestellt. Eine entscheidende Frage, die sich nun ergibt, betrifft die diagnostischen Merkmale, aufgrund derer eine Äußerung oder ein Äußerungsteil der Haupt- oder der Nebenstruktur zugewiesen werden kann. Sowohl Klein/von Stutterheim (1992) und von Stutterheim (1989) als auch Simons et al. (2011) nennen als zentrales Kriterium zur Unterscheidung zwischen Haupt- und Nebenstruktur die Frage, ob eine Äußerung bzw. ein Äußerungsteil relevant bezogen auf die aktuelle Q ist. Diese Sicht findet in Definition (25) in Abschnitt 2.1.2 nach Simons et al. (2011) ihren Niederschlag. Probleme entstehen nun allerdings, wenn es sich bei der Relevanz bezogen auf die jeweilige Q um das einzig wirksame Kriterium für die Unterscheidung zwischen [+at-issue]- und [-at-issue]-Inhalten handelt, da dieses Testverfahren zum Teil unscharf ist. Beispielsweise hilft die Relevanz bezogen auf Q nur beschränkt bei der Zuordnung einer Äußerung zur Haupt- oder Nebenstruktur, wenn Q implizit ist. So wurde bezogen auf das Beispiel (72) in Abschnitt 2.2.3, hier wiederholt als (87), dafür argumentiert, dass der Kausalsatz zur Hauptstruktur gehört, da mit ihm eine implizite Frage Q' = Warum nicht? beantwortet wird. Hierbei handelt es sich jedoch um eine rein intuitive Einsicht, welche mithilfe des einzigen uns bisher zur Verfügung stehenden Kriteriums nicht weiter überprüfbar ist.

Q: Hat Peter gestern die Klausur mitgeschrieben?

B: Nein, er konnte sie nicht mitschreiben, weil er krank ist.

Problematisch ist zudem die Tatsache, dass selbst in Fällen, in denen Q explizit geäußert ist, nicht immer ganz klar ist, welche Äußerungsteile der Beantwortung von Q dienen. In dem folgenden Beispiel kann Q beispielsweise entweder durch den semifaktiven Komplementsatz beantwortet werden, dann wäre B traurig, weil sie durchgefallen ist, oder aber durch den Gesamtsatz, in diesem Fall wäre B dann traurig, weil sie von dem Nichtbestehen erfahren hat.

Q: Warum bist du so traurig?

B: Ich habe gerade erfahren, dass ich durchgefallen bin.

Da es sich bei der Relevanz bezogen auf die jeweils aktuelle Q demnach um ein sehr vages Konzept handelt, wäre es wünschenswert, wenn weitere distinktive Merkmale, die [-at-issue]-Inhalte auszeichnen, ausgemacht werden könnten. Roberts et al. (2009) und Simons et al. (2011) beobachten nun, dass sich semantische Operatoren 


\section{Typische Eigenschaften der Nebenstruktur}

wie beispielsweise Negation oder Frageoperatoren nur auf die Hauptstruktur einer Äußerung beziehen und alle [-at-issue]-Inhalte ignorieren. Dies bedeutet, dass die Nebenstruktur immer außerhalb des Skopus von derartigen semantischen Operatoren interpretiert wird. Daraus ergeben sich nun zwei zentrale Eigenschaften, die sich alle [-at-issue]-Inhalte nach Roberts et al. (2009) und Simons et al. (2011) teilen. Zum einen projizieren sie, wenn sie unter einem sonst inferenztilgenden Operator eingebettet sind, da sich der Operator nicht auf den in ihnen ausgedrückten Inhalt bezieht. Ein weiteres wichtiges Merkmal von [-at-issue]-Inferenzen besteht darin, dass ihnen nur mithilfe bestimmter Diskursstrategien widersprochen werden kann. Ein direkter Widerspruch wie Nein bezieht sich ebenfalls nur auf die Hauptstruktur und lässt alle [-at-issue]-Inhalte unberücksichtigt. Die Beobachtung, dass die Inhalte, die direkt negierbar sind, die höchste kommunikative Relevanz besitzen, findet sich bereits bei Posner (1972) und wird von u.a. Bartsch (1978) zur Unterscheidung von Haupt- und Nebeninformationen genutzt. ${ }^{21}$

Da die beiden Eigenschaften, Projektion unter Einbettung und die Immunität gegenüber direkten Widerspruchsstrategien, nicht auf [+at-issue]-Inhalte, die im Einflussbereich semantischer Operatoren stehen, zutreffen, können sie als Tests zur Ermittlung der Nebenstruktur verwendet werden. Aus diesem Grund wird im Folgenden überprüft, ob diese beiden Merkmale auf die im vorherigen Abschnitt untersuchten Nebensatztypen zutreffen. Zu erwarten ist dabei, dass die Nebensatztypen der Gruppe 2, zu denen faktive Komplementsätze, Temporalsätze, appositive Relativsätze und restriktive Relativsätze mit einem definiten Antezedens zählen, beide relevanten Eigenschaften uneingeschränkt aufweisen. Die Nebensatztypen der Gruppe 1 dagegen, also Komplementsätze von semifaktiven und Brückenverben, weil-Sätze und restriktive Relativsätze mit einem indefiniten Antezedens, sollten die beiden Eigenschaften nur in solchen Kontexten zeigen, in denen sie [-at-issue]-Inhalt ausdrücken. ${ }^{22}$

Das folgende Kapitel ist in zwei Teile gegliedert: Im Zentrum von Abschnitt 3.1 wird das Projektionsverhalten der relevanten Nebensatztypen stehen. In Abschnitt 3.2 wird anschließend untersucht, auf welche Weise den verschiedenen Nebensatztypen widersprochen werden kann. Hierzu wurde eine Fragebogenstudie durchgeführt, deren Ergebnisse in Abschnitt 3.2.3 nach einem kurzen Überblick über die relevan-

${ }^{21}$ Zudem zeigt Posner (1972), dass nur kommunikativ relevante Bedeutungsaspekte mit Wirklich? erfragt werden können (vgl. dazu auch Bentzen 2009, die ebenfalls [+at-issue]-Inhalte durch Erfragbarkeitstests ermittelt).

${ }^{22}$ Brandt (1996) verwendet eine spezielle Form des Kommentartests zur Unterscheidung zwischen Vordergrund- und Hintergrundinformationen, bei dem es darum geht, dass mit dennoch ausschließlich Informationen des Vordergunds aufgegriffen werden können. Dieser Test wird im Folgenden aus mehreren Gründen nicht verwendet: Zum einen dient er bei Brandt (1996) im Rahmen ihrer zweidimensionalen Reliefgebung ausdrücklich nicht der Ermittlung von quaestiobezogenen Äußerungen, sondern von kommunikativ relevanten Informationen. Zum anderen ist die Validität des Tests fragwürdig. So bemerkt Pittner (1999, 207f), dass die in Brandt (1996) vorgestellten intuitiven Ergebnisse nicht von allen Sprechern geteilt werden, während Holler (2009) zu bedenken gibt, dass es sich bei den beobachteten Effekten auch um Linearitätseffekte handeln könnte, die nicht mit der Informationsgewichtung zusammenhängen. 
ten bisherigen Untersuchungen in Abschnitt 3.2.2 vorgestellt werden.

Beide Eigenschaften, Projektion unter Einbettung und Immunität gegenüber einem direkten Widerspruch, gelten traditionell als distinktive Merkmale von Präsuppositionen. In den folgenden Abschnitten werden wir jedoch sehen, dass es sich um keine geeigneten Tests zur Ermittlung präsupponierter Inhalte handelt, da beide Eigenschaften auf alle [-at-issue]-Inhalte und nicht nur auf Präsuppositionen zutreffen (zum Verhältnis von Präsuppositionen und dem Konzept der At-issueness vgl. Kapitel 4).

\subsection{Projektion}

\subsubsection{Grundlagen}

Nebensätze unterscheiden sich darin, ob sie unter Einbettung projizieren oder nicht. Projektion bezeichnet dabei das Überleben einer Inferenz, wenn diese im Skopus eines sonst inferenztilgenden Operators steht (vgl. u.a. Geurts 1999, Roberts et al. 2009 und Simons et al. 2011). Beispielsweise beinhaltet der Satz in (89) (mindestens) zwei Inferenzen: (i) Jenny hat die roten Schuhe gekauft und (ii) sie bereut es. Das Zeichen , $\rightsquigarrow$ ' wird im Folgenden verwendet, um anzuzeigen, dass es sich bei B um eine Inferenz von A handelt.

(89) Jenny bereut, dass sie die roten Schuhe gekauft hat.

(i) $\rightsquigarrow$ Jenny hat die roten Schuhe gekauft.

(ii) $\rightsquigarrow$ Jenny bereut den Schuhkauf.

Wird die initiale Äußerung nun negiert wie in (90), bleibt nur Inferenz (i) bestehen, wohingegen Inferenz (ii) von der Negation betroffen ist.

(90) Jenny bereut nicht, dass sie die roten Schuhe gekauft hat.

(i) $\rightsquigarrow$ Jenny hat die roten Schuhe gekauft.

(ii) $\not \rightarrow$ Jenny bereut den Schuhkauf.

Die Projektion bestimmter Bedeutungsaspekte wird klassischerweise im Kontext von Präsuppositionen erforscht. In zahlreichen Arbeiten gilt Projektion unter Einbettung als zentrale Eigenschaft von präsupponierten Inhalten, die typischerweise erhalten bleiben, auch wenn der Präsuppositionsauslöser im Skopus eines inferenztilgenden Operators wie Negation steht. Konstruktionen wie die in (91) aufgelisteten bilden die sogenannte P-Familie, welche typischerweise als Test für Präsuppositionen verwendet wird (so zum Beispiel in Bentzen 2009, Chierchia/McConnell-Ginet 1990, Geurts 1999 und Karttunen 1973). Wenn es sich bei B um eine Präsupposition von A handelt, sollte B dementsprechend auch dann erhalten bleiben, wenn A unter einem Negationsoperator (dargestellt in (91-a)), einem Frage- (vgl. (91-d)) oder einem epistemischen Operator (vgl. (91-b)) eingebettet ist, oder, wenn A das Antezedens eines Konditionalgefüges bildet wie in (91-c). 
Wenn A $\rightsquigarrow \mathrm{B}$, dann:

a. $\neg \mathrm{A} \rightsquigarrow \mathrm{B}$

b. Es ist möglich, dass $\mathrm{A} \rightsquigarrow \mathrm{B}$

c. $\mathrm{A} \rightarrow \mathrm{C} \rightsquigarrow \mathrm{B}$

d. $\mathrm{A} ? \rightsquigarrow \mathrm{B}$

Wenn wir unser initiales Beispiel (89) betrachten, lässt sich feststellen, dass die Inferenz (i) in allen Konstruktionen der P-Familie überlebt:

(92) a. Jenny bereut nicht, dass sie die roten Schuhe gekauft hat.

b. Es ist möglich, dass Jenny bereut, dass sie die roten Schuhe gekauft hat.

c. Wenn Jenny bereut, dass sie die roten Schuhe gekauft hat, zeigt sie es zumindest nicht.

d. Bereut Jenny, dass sie die roten Schuhe gekauft hat?

$\rightsquigarrow$ Jenny hat die roten Schuhe gekauft.

Das Ergebnis deckt sich mit der gängigen Annahme, dass bereuen als faktives Verb ein präsupponiertes Komplement einbettet. Das propositionale Komplement eines nicht-faktiven Verbs wie glauben dagegen projiziert nicht, wie die folgenden Sätze zeigen. Bei keinem der Beispielsätze können wir davon ausgehen, dass Jenny die Schuhe gekauft hat.

(93) Evi glaubt, dass Jenny die roten Schuhe gekauft hat.

a. Evi glaubt nicht, dass Jenny die roten Schuhe gekauft hat.

b. Es ist möglich, dass Evi glaubt, dass Jenny die roten Schuhe gekauft hat.

c. Wenn Evi glaubt, dass Jenny die roten Schuhe gekauft hat, kennt sie ihre Tochter schlecht.

d. Glaubt Evi, dass Jenny die roten Schuhe gekauft hat?

$\not \sim$ Jenny hat die roten Schuhe gekauft.

Auch die durch eine definite NP ausgelöste Existenzpräsupposition projiziert in den Konstruktionen der P-Familie. Exemplarisch ist dies in den folgenden Sätzen belegt, die alle implizieren, dass es in dem entsprechenden Äußerungskontext eine (reale oder nicht-reale) Entität gibt, auf die die Beschreibung kaputter Motor zutrifft.

(94) Der kaputte Motor verursacht Probleme.

a. Der kaputte Motor verursacht keine Probleme.

b. Verursacht der kaputte Motor Probleme?

$\rightsquigarrow$ Es gibt einen kaputten Motor.

Wir haben bereits gesehen, dass Projektion traditionell als eine typische Eigenschaft von Präsuppositionen betrachtet und die Sätze der P-Familie als Präsuppositionstests verwendet werden. Jedoch projizieren nicht nur präsupponierte Bedeutungs- 
aspekte, wie u.a. Potts (2005), Potts (2011), Roberts et al. (2009) und Simons et al. (2011) beobachten. Beispielsweise bleibt der Inhalt eines appositiven Relativsatzes bei Anwendung der P-Familien-Tests bestehen, wie die folgenden Sätze zeigen.

Jap, der gerade in Indonesien ist, fährt gerne ans Meer.

a. Jap, der gerade in Indonesien ist, fährt nicht gerne ans Meer.

b. Es ist möglich, dass Jap, der gerade in Indonesien ist, gerne ans Meer fährt.

c. Wenn Jap, der gerade in Indonesien ist, gerne ans Meer fährt, dann mag er sicher die Ostsee.

d. Fährt Jap, der gerade in Indonesien ist, gerne ans Meer?

$\rightsquigarrow$ Jap ist gerade in Indonesien.

Obwohl appositive Relativsätze unter Einbettung projizieren, sind sie dennoch nicht präsupponiert. Dies zeigt sich daran, dass ein appositiver Relativsatz eindeutig dazu verwendet wird, eine neue Information einzuführen. Sein Gebrauch unterliegt also keiner Common-Ground-Forderung, wie sie typischerweise für Präsuppositionen gilt (vgl. dazu Kapitel 4). Mehr noch, u.a. Holler (2005, 54) und Roberts et al. (2009) zeigen, dass die Äußerung eines appositiven Relativsatzes sogar inakzeptabel ist, wenn die Proposition wie in den folgenden beiden Beispiel bereits vorerwähnt wurde (Beispiel (96-a) nach Holler 2005, 54):

a. \#Ein Mann küsste eine Frau und diese Frau, die er übrigens küsste, war verheiratet.

b. Jap ist gerade in Indonesien. \#Jap, der (übrigens) gerade in Indonesien ist, fährt gerne ans Meer.

Obwohl der appositive Relativsatz unter dem syntaktischen Skopus von Negation und anderen implikationstilgenden Operatoren heraus projiziert, ist er demnach trotzdem nicht präsupponiert. Dasselbe gilt für expressive Bedeutungen. Beispielsweise überlebt die expressive Inferenz, dass $S$ Tom für einen Idioten hält, in allen Sätzen der P-Familie, wie aus den Beispielen in (97) ersichtlich wird.

Dieser Idiot Tom ist heute mit Kochen dran.

a. Dieser Idiot Tom ist heute nicht mit Kochen dran.

b. Es ist möglich, dass dieser Idiot Tom heute mit Kochen dran ist.

c. Wenn dieser Idiot Tom heute mit Kochen dran ist, gehe ich ins Restaurant.

d. Ist dieser Idiot Tom heute mit Kochen dran?

$\rightsquigarrow \mathrm{S}$ hält Tom für einen Idioten.

Auch Expressiva sind nicht präsupponiert, obwohl sie projizieren. Genau wie im Falle des appositiven Relativsatzes in Beispiel (96-b) ist auch die Verwendung eines 
expressiven Ausdrucks nicht akzeptabel, wenn dessen Inhalt vorerwähnt ist: ${ }^{23}$

Tom ist ein Idiot. \#Dieser Idiot Tom ist heute mit Kochen dran.

Die Tatsache, dass auch nicht-präsupponierte Bedeutungsaspekte unter Einbettung projizieren, zeigt, dass Projektion kein distinktives Merkmal von Präsuppositionen ist. Demnach bilden die Sätze der P-Familie in (91) keine geeignete Strategie, um Präsuppositionen zu ermitteln. Vielmehr zeigen die Beispiele in (95) und (97), dass projizierende Bedeutungsaspekte eine große Spannbreite an unterschiedlichen Inferenzen umfassen. In Kapitel 4 wird dafür argumentiert, dass Präsuppositionen eine Untermenge dieser Gruppe bilden. Demnach können die Sätze der P-Familie auch nicht als hinreichender Präsuppositionstest verwendet werden.

Jüngst haben Roberts et al. (2009) und Simons et al. (2011) Projektion als eine pragmatische Eigenschaft analysiert, die mit der Diskursstruktur einer Äußerung zusammenhängt. Basierend auf der Beobachtung, dass projizierende Ausdrücke nicht immer präsupponiert sind, schließen sie, dass alle projizierenden Bedeutungsaspekte einen besonderen Diskursstatus haben: Sie sind [-at-issue]. Ausgehend von der Annahme, dass sich Operatoren wie Negation auf die Hauptstruktur einer Äußerung beziehen, nehmen Roberts et al. (2009) und Simons et al. (2011)an, dass [-atissue]-Inhalte von ihnen ignoriert werden und dementsprechend trotz Einbettung überleben, d.h. projizieren. Die Hypothese darüber, was warum projiziert, ist im Folgenden nach Simons et al. (2011, 6) dargestellt:

\section{Hypotheses about what projects and why}

i. All and only those implications of (embedded) sentences which are notat-issue relative to the Question Under Discussion in the context have the potential to project.

ii. Operators (modals, negation, etc.) target at-issue content.

\footnotetext{
${ }^{23}$ In der Annahme, dass Expressiva nicht präsupponiert sind, folge ich u.a. Potts (2005), der expressive Bedeutungen als konventionelle Implikaturen analysiert, welche keine Gegebenheitsanforderung an den Common Ground stellen. Über diesen Punkt herrscht in der Forschungsliteratur jedoch kein Konsens. So argumentiert insbesondere Schlenker (2007), dass Expressiva indexikalische Präsuppositionsauslöser sind, welche im Unterschied zu klassischen Präsuppositionsauslösern systematisch informativ sind (vgl. dazu auch Sauerland 2007). Im Sinne von Lewis (1979) muss die durch einen expressiven Ausdruck ausgelöste Präsupposition nach Schlenker (2007) systematisch akkommodiert werden, aus diesem Grund spricht er von „,self-fulfilling presuppositions". Ob man Expressiva nun aber in die Klasse der Präsuppositionsauslöser zählt oder nicht, hängt letztendlich davon ab, welcher Präsuppositionsbegriff der jeweiligen Analyse zugrunde liegt. Es gibt zahlreiche und zum Teil sehr unterschiedliche Definitionen des Begriffs, von denen einige in Abschnitt 4 diskutiert werden. Fest steht, dass Präsuppositionen auch informativ sein können, ihr Inhalt also zum Äußerungszeitpunkt noch nicht Teil des Common Grounds sein muss. Es ist jedoch fraglich, welches Merkmal sich alle Präsuppositionen teilen, wenn einige Präsuppositionen sogar informativ sein müssen, wie es aus der Analyse von Schlenker (2007) folgt. Aus diesem Grund wird im Folgenden an der Annahme festgehalten, dass Expressiva nicht präsupponiert sind.
} 
Nimmt man nun mit Roberts et al. (2009) und Simons et al. (2011)an, dass [-atissue]-Bedeutungen unter Einbettung projizieren, können die Sätze der P-Familie anstatt als Präsuppositionstest als Test zur Ermittlung von At-issueness verwendet werden. Diese Annahme wurde u.a. durch die experimentelle Untersuchung von Xue/Onea (2011) bestätigt und wird auch dem folgenden Abschnitt zugrunde liegen, in dem das Projektionsverhalten der in dieser Arbeit diskutierten Nebensatztypen untersucht werden soll. Dabei ist zu erwarten, dass alle Nebensatztypen, für die in Abschnitt 2.2 gezeigt wurde, dass sie nicht Teil der Hauptstruktur sein können, also die Nebensatztypen von Gruppe 2 (vgl. (86)), unter Einbettung projizieren. Diese Überlegung ist in Hypothese I in (100) zusammengefasst und soll im folgenden Abschnitt überprüft werden. Aus der Sicht auf Projektion als ein diskursstrukturelles Phänomen folgt zudem, dass Projektion kontextabhängig ist, da die einem Diskurs jeweils zugrundeliegende Frage Q bestimmt, welche Äußerungsteile zur Haupt- bzw. zur Nebenstruktur gehören. Nebensätze, die je nach Kontext entweder zur Hauptoder zur Nebenstruktur zählen, also die Nebensatztypen von Gruppe 1 aus (86), sollten ebenfalls unter Einbettung projizieren, wenn sie für die Beantwortung der jeweils aktuellen Q nicht relevant sind. Diese Überlegungen sind in den Hypothesen I und II in (100) zusammengefasst:

$$
\begin{aligned}
& \text { Hypothesen zum Projektionsverhalten abhängiger Sätze } \\
& \text { I Nebensatztypen der Gruppe } 2 \text { projizieren immer aus dem Skopus eines } \\
& \text { inferenztilgenden Operators heraus. } \\
& \text { II Nebensatztypen der Gruppe } 1 \text { projizieren nicht, wenn sie [+at-issue]- } \\
& \text { Inhalt ausdrücken. }
\end{aligned}
$$

\subsubsection{Projektionsverhalten von abhängigen Sätzen}

Beginnen wir mit der Überprüfung von Hypothese I. In Abschnitt 2.2 wurde gezeigt, dass Komplementsätze von faktiven Verben, temporale Adverbialsätze, appositive Relativsätze und restriktive Relativsätze mit einem definiten Antezedens nicht dazu geeignet sind, [+at-issue]-Inhalt auszudrücken. Da es sich dabei um eine kontextuell unabhängige Eigenschaft handelt, wurden alle vier Satztypen als konventionelle Markierungen für die Nebenstruktur analysiert. Bringt man diese Ergebnisse mit Hypothese (99) nach Simons et al. (2011) zusammen, so lautet die Vorhersage, dass diese vier Nebensatztypen unabhängig vom Kontext unter Einbettung projizieren. Wir haben in Beispiel (92) bereits gesehen, dass ein durch ein faktiv-emotives Verb wie bereuen eingebetteter Komplementsatz in allen Konstruktionen der P-Familie projiziert. Zudem lässt sich feststellen, dass dies auch für Fälle gilt, in denen der Komplementsatz eine bezogen auf Q relevante Information ausdrückt. In Beispiel (59), hier wiederholt als (101), drückt der eingebettete Satz eindeutig [+at-issue]Inhalt aus, weswegen das Beispiel nicht wohlgeformt ist. Wenden wir trotzdem den Negationstest an, lässt sich feststellen, dass auch hier die Nebensatzproposition projiziert. 
Q: Wo ist denn Homer?

B: \#Marche bereut, dass sie ihn in den Kwik-E-Markt geschickt hat.

B':\#Marche bereut nicht, dass sie ihn in den Kwik-E-Markt geschickt hat.

$\rightsquigarrow$ Marche hat Homer in den Kwik-E-Markt geschickt.

Aus der Tatsache, dass die Komplementsatzproposition unter Einbettung projiziert, können wir schließen, dass der Nebensatz als [-at-issue] analysiert wird, da er ansonsten im semantischen Skopus des Negationsoperators stehen würde. Dies aber zeigt abermals, dass wir es mit einer konventionellen Markierung der Nebenstruktur zu tun haben. Denn obwohl der Satz rein inhaltlich eindeutig eine bezogen auf Q relevante Information ausdrückt, wird er als [-at-issue] interpretiert und projiziert deswegen. Die Inakzeptabilität des Beispiels entsteht dann dadurch, dass ein Satz, der konventionell als [-at-issue] markiert ist, aufgrund seines Inhalts eigentlich zur Hauptstruktur gehören müsste.

Dasselbe gilt für die andere Gruppe an faktiven Verben, nämlich Berücksichtigungsverben. Wird das Verb bereuen in den Beispielen (59) und (101) durch ignorieren ersetzt, lässt sich ebenfalls feststellen, dass die Nebensatzproposition unter Einbettung projiziert, und zwar auch dann, wenn der Satz wie in dem folgenden Beispiel eine bezogen auf Q relevante Information ausdrückt:

Q: Wie ist denn das Wetter?

$\mathrm{B}$ : \#Marche ignoriert, dass es regnet, und ist schwimmen gegangen.

B':\#Marche ignoriert nicht, dass es regnet, und geht ins Hallenbad.

$\rightsquigarrow$ Es regnet.

Wie sieht es nun mit temporalen Adverbialsätzen aus? Dies soll hier exemplarisch für die drei temporalen Subjunktionen als, bevor und nachdem in dem komplexen Satz (103) untersucht werden. Wird der Satz entsprechend der Konstruktionen der PFamilie umgeformt, können wir feststellen, dass die Proposition des Temporalsatzes wie auch die des faktiven Komplementsatzes in (92) aus der Einbettung heraus projiziert. In allen vier Testkonstruktionen (103-a) bis (103-d) bleibt die Inferenz, dass eine Leiche gefunden wurde, bestehen. Auch hier ist die auf Grundlage von Simons et al. (2011) getroffene Hypothese (100) demnach zutreffend.

(103) Die Kommissarin erreichte den Tatort, als/nachdem/bevor die Leiche gefunden wurde.

a. Die Kommissarin erreichte den Tatort nicht, als/bevor/nachdem die Leiche gefunden wurde.

b. Es ist möglich, dass die Kommissarin den Tatort erreichte, als/bevor/ nachdem die Leiche gefunden wurde.

c. Wenn die Kommissarin den Tatort erreichte, als/bevor/nachdem die Leiche gefunden wurde, dann hat sie sich sehr beeilt.

d. Hat die Kommissarin den Tatort erreicht, als/bevor/nachdem die Leiche gefunden wurde? 
$\rightsquigarrow$ Die Leiche wurde gefunden.

Für Temporalsätze gilt ebenfalls, dass sie unabhängig vom Kontext projizieren. Auch in dem folgendem Beispiel, in dem der Temporalsatz [+at-issue]-Inhalt ausdrückt, weswegen die Äußerung nicht wohlgeformt ist, überlebt die Nebensatzproposition unter Einbettung. Dies zeigt abermals, dass Temporalsätze konventionell zur Nebenstruktur gehören, und dass diese Eigenschaft kontextuell nicht uminterpretiert werden kann. Die Inakzeptabilität der beiden Äußerungen B und B' in diesem Kontext resultieren dann aus einem Mismatch zwischen konventionell als [-at-issue] markierter Form und aktuellem Gebrauch als Träger der Hauptinformation.

Q: Hat Peter das Auto gekauft?

B: \#Peter war im Kino, bevor er das Auto gekauft hat.

B':\#Peter war nicht im Kino, bevor er das Auto gekauft hat.

$\rightsquigarrow$ Peter hat das Auto gekauft.

Dass Hypothese I auch auf appositive Relativsätze zutrifft, belegen die Sätze in Beispiel (95) im vorherigen Abschnitt, in denen die im Relativsatz ausgedrückte Proposition trotz Einbettung überlebt. Wie auch bei faktiven Komplementsätzen und bei Temporalsätzen handelt es sich dabei auch im Falle der appositiven Relativsätze um eine kontextunabhängige Eigenschaft. In Beispiel (75) in Abschnitt 2.2.4, hier wiederholt als (105), wurde bereits gezeigt, dass die Verwendung eines appositiven Relativsatzes in Kontexten, in denen er [+at-issue]-Inhalt ausdrückt, nicht wohlgeformt ist. Wenden wir dennoch den Negationstest an (vgl. B'), wird ersichtlich, dass die Nebensatzproposition erhalten bleibt, das heißt projiziert.

Q: Wo ist eigentlich Jap im Moment? Ich habe ihn schon lange nicht mehr gesehen.

B: \#Jap, der gerade in Indonesien ist, backt gerne Kuchen.

B':\#Jap, der gerade in Indonesien ist, backt nicht gerne Kuchen.

$\rightsquigarrow$ Jap ist gerade in Indonesien.

Auch dieses Beispiel zeigt, dass wir es mit einer konventionellen Markierung der Nebenstruktur zu tun haben. Obwohl der Relativsatz eindeutig bezogen auf Q relevante Informationen enthält, wird er als [-at-issue] interpretiert und demnach von dem Negationsoperator ignoriert. Dass die Äußerungen B und B' in diesem Kontext nicht wohlgeformt sind, resultiert dann abermals daraus, dass Form und Funktion des Nebensatzes nicht zusammenpassen.

Betrachten wir abschließend noch restriktive Relativsätze mit einem definiten Antezedens. Wie zu erwarten, projiziert die eingebettete Proposition in den Konstruktionen der P-Familie:

(106) Dominik liebt die Frau, die bei der Polizei arbeitet.

a. Dominik liebt nicht die Frau, die bei der Polizei arbeitet.

b. Es ist möglich, dass Dominik die Frau, die bei der Polizei arbeitet, 
liebt.

c. Wenn Dominik die Frau, die bei der Polizei arbeitet liebt, sollte er besser keine krummen Dinger drehen.

d. Liebt Dominik die Frau, die bei der Polizei arbeitet?

$\rightsquigarrow$ Es gibt eine (kontextuell relevante) Frau, die bei der Polizei arbeitet.

Wir können folglich festhalten, dass Hypothese I zutreffend ist. Alle Nebensatztypen, die konventionell zur Nebenstruktur zählen, projizieren unter Einbettung. Zudem handelt es sich dabei um eine kontextunabhängige Eigenschaft: Zum einen sind die Nebensätze der Gruppe 2 prinzipiell in Kontexten, in denen sie [+at-issue]-Inhalt ausdrücken, ausgeschlossen, so dass sie immer, wenn sie verwendet werden, zur Nebenstruktur gehören und dementsprechend von semantischen Operatoren ignoriert werden. Aber selbst, wenn man nicht-wohlgeformte Beispiele wie (101), (102) oder (104), in denen der Nebensatz die jeweilige Q beantwortet, betrachtet, lässt sich feststellen, dass die Nebensatzproposition unter Einbettung projiziert. Dies wurde als weitere Evidenz dafür gewertet, dass die Nebensatztypen der Gruppe 2 konventionell zur Nebenstruktur gehören.

Betrachten wir nun im Vergleich zu den bisher untersuchten Nebensätzen die Nebensatztypen der Gruppe 1, also solche Nebensätze, welche auch zur Hauptstruktur gehören können. Hypothese II sagt voraus, dass das Projektionsverhalten dieser Nebensatztypen vom jeweiligen Diskurskontext abhängt. Beginnen wir mit den Komplementen von Brückenverben. In Beispiel (93) wurde bereits gezeigt, dass ein durch ein Brückenverb eingebetteter Komplementsatz unter Einbettung nicht überlebt. Daraus können wir schließen, dass Komplementsätze von Brückenverben, wenn sie wie in (93) durch den Kontext nicht näher spezifiziert sind, als Teil der Hauptstruktur analysiert werden. Als solcher stehen sie im Einflussbereich von semantischen Operatoren und können in den Sätzen der P-Familie nicht überleben. Wie wir in Abschnitt 2.2 gesehen haben, können Brückenverben aber auch einen Komplementsatz einbetten, der [-at-issue] ist (vgl. Beispiel (48)). In dem folgenden Beispiel ist die Beantwortung der Frage ?p = War Marche immer treu? nicht relevant bezogen auf Q. Der Nebensatz gehört demnach zur Nebenstruktur und interessanterweise projiziert er aus der Einbettung heraus.

Q: Warum ist Marche so traurig?

B: Homer glaubt nicht, dass sie immer treu war. $\rightsquigarrow$ Marche war immer treu.

Aus der Sicht auf Projektion als ein diskursstrukturelles Phänomen folgt, dass Projektion kontextabhängig ist, da die einem Diskurs jeweils zugrundeliegende Frage Q bestimmt, welche Äußerungsteile zur Haupt- bzw. zur Nebenstruktur gehören. Das Projektionsverhalten von Nebensätzen, die wie nicht-faktive Komplementsätze je nach Kontext entweder zur Haupt- oder zur Nebenstruktur zählen, ist demnach kontextabhängig. Ist der Nebensatz wie in Beispiel (107) bezogen auf Q nicht relevant, wird er von semantischen Operatoren ignoriert und projiziert. Gehört er 
dagegen zur Hauptstruktur, bezieht sich der Negationsoperator auch auf die subordinierte Proposition, welche deswegen nicht überlebt. Wird der Satz wie in Beispiel (93) kontextlos geäußert, projiziert er nicht. Daraus können wir schließen, dass die Default-Lesart für nicht-faktive Komplementsätze so aussieht, dass diese Teil der Hauptstruktur sind.

Wie nicht-faktive Komplementsätze überleben auch weil-Sätze nicht unter Einbettung. In dem folgenden Beispiel lässt der initiale Satz die Inferenz zu, dass Homer verschlafen hat. Durch die Folgesätze, in denen der Kausalsatz jeweils im Skopus eines inferenztilgenden Operators steht, wird diese Inferenz dagegen nicht ausgelöst.

(108) Homer hat das Meeting verpasst, weil er verschlafen hat.

a. Homer hat das Meeting nicht verpasst, weil er verschlafen hat.

b. Es ist möglich, dass Homer das Meeting verpasst hat, weil er verschlafen hat.

c. Wenn Homer das Meeting verpasst hat, weil er verschlafen hat, wird er gefeuert.

d. Hat Homer das Meeting verpasst, weil er verschlafen hat?

$\not \rightarrow$ Homer hat verschlafen.

Wird ein weil-Satz dagegen in einem Kontext geäußert, in dem er [-at-issue] ist, können wir beobachten, dass die Nebensatzproposition projiziert wie in dem folgenden Beispiel:

Q: Warum ist Maria so traurig?

B: Es ist möglich, dass Peter sie verlassen hat, weil sie ihn betrogen hat. $\rightsquigarrow$ Maria hat Peter betrogen.

Allerdings ist es nicht immer einfach zu testen, ob ein weil-Satz projiziert, wenn er zur Nebenstruktur gehört. Zum einen ist es schwierig auszuschließen, dass der entsprechende Kausalsatz eine implizite Warum?-Frage beantwortet, in welchem Fall er dann nicht zur Neben-, sondern zur Hauptstruktur gehören würde. Außerdem muss sichergestellt sein, dass der Kausalsatz überhaupt im Skopus des jeweiligen inferenztilgenden Operators aus dem Matrixsatz steht. Beispielsweise könnte man bei der Betrachtung von Beispiel (110) schlussfolgern, dass der Kausalsatz projiziert, da die Inferenz, dass Peter krank ist, trotz Negation des Matrixsatzes überlebt. Dies würde im Widerspruch zu der in Abschnitt 2.2 bezogen auf Beispiel (72) getroffenen Aussage stehen: Denn wenn man annimmt, dass der Kausalsatz in dem folgenden Beispiel eine implizite Frage Q' = Warum nicht? beantwortet, dann ist der Satz Teil der Hauptstruktur und sollte demnach nicht projizieren.

Q: Hat Peter gestern die Klausur mitgeschrieben?

B: Nein, er konnte sie nicht mitschreiben, weil er krank ist.

Bei einer näheren Betrachtung des Beispiels wird jedoch klar, dass der Kausalsatz nicht im Skopus der Matrixnegation steht. Der Negationsoperator bezieht sich le- 


\section{Typische Eigenschaften der Nebenstruktur}

diglich auf die Matrixproposition, so dass wir es mit der semantischen Struktur weil $(p, \neg q)$ zu tun haben. Dass die Inferenz trotz Matrixnegation bestehen bleibt, ist demnach darauf zurückzuführen, dass der Satz gar nicht im Skopus der Negation liegt, und nicht darauf, dass die Inferenz aus dem Skopus der Negation heraus projiziert. Wir müssten die Äußerung gemäß der logischen Form $\neg$ weil $(p, q)$ interpretieren, um das Projektionsverhalten des Kausalsatzes testen zu können. Demnach bildet (110) kein geeignetes Beispiel zur Überprüfung des Projektionsverhaltens von [-at-issue]-Kausalsätzen. Dass das Projektionsverhalten von weil-Sätzen kontextabhängig ist, wird jedoch durch Beispiel (109) belegt. Wie im Falle der nichtfaktiven Komplementsätze gilt demnach auch bezogen auf weil-Sätze, dass diese als Default-Fall wie in Beispiel (108) als Teil der Hauptstruktur interpretiert werden und nicht projizieren. Legt der Kontext dagegen eine Lesart als Teil der Nebenstruktur fest, überlebt die Kausalsatzproposition unter Einbettung (vgl. Beispiel (109)).

Dass auch restriktive Relativsätze mit einem indefiniten Antezedens von semantischen Operatoren wie Negation betroffen sind und nicht überleben, belegen die folgenden Beispiele:

Dominik liebt eine Frau, die bei der Polizei arbeitet.

a. Dominik liebt keine Frau, die bei der Polizei arbeitet.

b. Es ist möglich, dass Dominik eine Frau, die bei der Polizei arbeitet, liebt.

c. Wenn Dominik eine Frau, die bei der Polizei arbeitet liebt, sollte er besser keine krummen Dinger drehen.

d. Liebt Dominik eine Frau, die bei der Polizei arbeitet?

$\Varangle \rightarrow$ Es gibt eine (kontextuell relevante) Frau, die bei der Polizei arbeitet.

Dass der Relativsatz hier nicht projiziert, hängt eindeutig mit dem indefiniten Antezedens zusammen, welches nach u.a. Heim (1982) und Kamp/Reyle (1993) nicht anaphorisch ist. Das Projektionsverhalten des restriktiven Relativsatzes in Beispiel (111) deckt sich zudem mit der Beobachtung, dass restriktive Relativsätze mit einem indefiniten Antezedens in der Lage sind, [+at-issue]-Inhalt auszudrücken. Offensichtlich ist dies wie bei nicht-faktiven Komplementsätzen und weil-Sätzen sogar die Default-Interpretation, da der Relativsatz in kontextlosen Äußerungen wie in Beispel (111) nicht projiziert.

Einen weiteren Nebensatztyp, der [+at-issue]-Inhalt ausdrücken kann, bilden die Komplemente von semifaktiven Prädikaten. Nach u.a. Karttunen (1971) unterscheiden sich semifaktive Verben von faktiven nun gerade darin, dass semifaktive Komplementsätze in Konditional- und Fragekonstruktionen ihre Faktivität verlieren, also, in anderen Worten, nicht projizieren. So löst Beispiel (112) zwar die Inferenz aus, dass sich Daniel verrechnet hat, diese Inferenz überlebt jedoch nicht, wenn der Satz in ein Frage- oder Konditionalsatzgefüge umgeformt wird wie in (113).

(112) Daniel hat entdeckt, dass er sich verrechnet hat.

$\rightsquigarrow$ Daniel hat sich verrechnet. 
(113) a. Hat Daniel entdeckt, dass er sich verrechnet hat?

b. Wenn Daniel entdecken sollte, dass er sich verrechnet hat, wäre das echt ärgerlich.

$\Varangle \rightarrow$ Daniel hat sich verrechnet.

Aus (113-a) kann nicht eindeutig inferiert werden, dass sich Daniel verrechnet hat, auch wenn diese Lesart natürlich leichter zugänglich ist (vgl. dazu auch Hooper/Thompson 1973, 480 und Karttunen 1971). Das Beispiel kann anders als eine entsprechende Äußerung mit einem faktiven Verb auch in Kontexten geäußert werden, in denen nicht feststeht, ob sich Daniel verrechnet hat, beispielsweise, wenn die Gesprächsteilnehmer wissen, dass ein von Daniel geschriebenes Programm nicht funktioniert, bislang aber unklar ist, ob dies auf einen Rechenfehler oder nicht zurückzuführen ist. Auch Beispiel (113-b) lässt die Lesart zu, dass es offen ist, ob sich Daniel verrechnet hat. Dies kann auch nicht auf das Modalverb sollen zurückgeführt werden: Wird ein faktives Verb in einer ähnlichen Konstruktion mit sollen verwendet, projiziert die Nebensatzproposition eindeutig wie in dem folgenden Beispiel:

Wenn Daniel bereuen sollte, dass er sein Auto verkauft hat, würde mich das wundern.

Genau in der fehlenden Projektion von semifaktiven Komplementsätzen in Frageund Konditionalkonstruktionen liegt ihre Abgrenzung zu faktiven Komplementsätzen begründet, was rechtfertigt, diese als zwei unterschiedliche Nebensatztypen zu klassifizieren.

Wird ein semifaktiver Komplementsatz dagegen als Teil der Nebenstruktur verwendet, projiziert er aus einem Konditionalsatzgefüge heraus, wie das folgende Beispiel belegt:

Q: Was ist denn passiert?

B: Hm, sollte Marche entdeckt haben, dass Bart raucht, würde das das Geschrei erklären.

Damit verhalten sich Komplementsätze, die durch ein semifaktives Verb eingebettet werden, wie durch Hypothese II vorhergesagt. Drücken sie [+at-issue]-Inhalt aus, projizieren sie nicht, andernfalls überleben sie unter Einbettung.

Wir können festhalten, dass Hypothese I aus (100) zutreffend ist: Alle Nebensatztypen, für welche in Abschnitt 2.2 gezeigt wurde, dass sie keine bezogen auf Q relevante Information ausdrücken können, projizieren unter Einbettung. Dabei handelt es sich um ein kontextunabhängiges Phänomen. Auch Hypothese II ist zutreffend, da die Nebensatztypen der Gruppe 1 tatsächlich nicht projizieren, wenn sie [+atissue]-Inhalt ausdrücken. Zudem konnten wir für die Sätze der Gruppe 1 eindeutig eine Default-Interpretation erkennen, da diese Nebensatztypen (mit Ausnahme der semifaktiven Komplementsätze), wenn sie kontextlos verwendet werden, im semantischen Skopus von inferenztilgenden Operatoren stehen und dementsprechend nicht projizieren. Nur wenn der Kontext eindeutig eine [-at-issue]-Lesart vorgibt, proji- 
zieren diese Nebensatztypen. Dies zeigt, dass die Sätze aus Gruppe 1 präferiert als Teil der Hauptstruktur analysiert werden. Alle Ergebnisse bezüglich des Projektionsverhaltens der hier diskutierten Nebensatztypen sind noch einmal in Tabelle 3.1 zusammengefasst.

\begin{tabular}{|l|c|}
\hline Nebensatztyp & Projektion \\
\hline \hline Komplementsatz, Brückenverb & $+/-$ \\
\hline Semifaktiver Komplementsatz & $+/-$ \\
\hline Faktiver Komplementsatz & + \\
\hline Temporaler Adverbialsatz & + \\
\hline Adverialsatz mit weil & $+/-$ \\
\hline Appositiver Relativsatz & + \\
\hline Restriktiver Relativsatz, definites Antezedens & + \\
\hline Restriktiver Relativsatz, indefinites Antezedens & $+/-$ \\
\hline
\end{tabular}

Tabelle 3.1.: Projektionsverhalten von Nebensätzen

\subsection{Widerspruchsstrategien}

\subsubsection{Grundlagen}

Neben der Projektion unter Einbettung teilen sich [-at-issue]-Inferenzen eine weitere Eigenschaft. Dabei geht es darum, dass der Wahrheitsgehalt einer Aussage, die zur Nebenstruktur gehört, nur mithilfe spezieller Diskursstrategien angefochten werden kann, wie bereits Bartsch (1978) und Posner (1972) beobachten. Betrachten wir dazu ein Beispiel, frei übersetzt nach Roberts et al. (2009, 4). Der folgende Beispielsatz lässt die Inferenz m zu, derzufolge Hörer B die Angewohnheit hat bzw. hatte, zum Frühstück Bier zu trinken. Diese Inferenz m wird nicht angefochten, wenn B mit einem direkten Widerspruch antwortet. Vielmehr verpflichtet der direkte Widerspruch B auf die Wahrheit von $\mathrm{m}$. Möchte B den Inhalt von $\mathrm{m}$ anfechten, muss er dies indirekt tun, wie in (116-b) nach Roberts et al. $(2009,4)$ definiert.

A: Hast du aufgehört, Bier zum Frühstück zu trinken?

$\rightsquigarrow \mathrm{m}=\mathrm{Du}$ hattest die Angewohnheit, Bier zum Frühstück zu trinken.

a. Direkter Widerspruch: Nein

b. Indirekter Widerspruch: Hey, warte mal!, Was meinst du, ...

Eingeführt durch Posner (1972) und Shannon (1976) und bekannt gemacht durch von Fintel (2004b), der als Diskursstrategie zur Erfragung von Präsuppositionen Hey, wait a minute! (I didn't knew that $x$ ) nennt, wird der sogenannte Hey, wait a minute!-Test ähnlich wie die Sätze der P-Familie traditionell als Präsuppositionstest verwendet (so beispielsweise in Bartsch 1978 oder Pasch et al. 2003, 154). Häufig wird auch gar nicht zwischen Präsuppositionen und [-at-issue]-Inferenzen 
unterschieden - beispielsweise gehen Bartsch (1978) und Hartmann (1984) davon aus, dass Nebensätze grundsätzlich immer präsupponiert sind und keine Hauptinformationen ausdrücken. Dass dies jedoch nicht zutreffend ist, wurde bereits mehrfach gezeigt (zum Verhältnis zwischen dem Konzept der At-issueness und Präsuppositionen vgl. Kapitel 4).

Der Hey, wait a minute!-Test basiert auf der Überlegung, dass präsupponierte Inhalte anders erfragt bzw. hinterfragt werden als assertierte. Beispielsweise kann dem durch das Brückenverb glauben eingebetteten Komplementsatz aus Beispiel (93), hier wiederholt als (117), direkt widersprochen werden. Dass sich B's Äußerung auf den Inhalt des Komplementsatzes beziehen kann, wird daran deutlich, dass eine korrigierende Weiterführung möglich ist.

A: Evi glaubt, dass Jenny die roten Schuhe gekauft hat.

B: Nein, das stimmt nicht. Jenny hat die GRÜnen gekauft.

Dagegen bleibt die Wahrheit eines präsupponierten Komplementsatzes durch einen direkten Widerspruch unangefochten. Aus diesem Grund ist eine Weiterführung wie in B in Beispiel (118) nicht wohlgeformt. Soll dem präsupponierten Komplementsatz widersprochen werden, kommt dazu nur eine indirekte Form des Widerspruchs wie in $\mathrm{B}^{\prime}$ in Frage. ${ }^{24}$

A: Jenny bereut, dass sie die roten Schuhe gekauft hat.

B: Nein, das stimmt nicht. \#Sie hat die GRÜnen gekauft.

B': Hey, warte mal! - Sie hat doch die GRÜnen gekauft!

Dasselbe gilt für definite NPn, welche als Präsuppositionsauslöser ebenfalls nur mithilfe der Hey, wait a minute!-Strategie, angefochten werden können, wie das berühmte Beispiel in (119) nach Russell (1905) zeigt. Ein direkter Widerspruch wie in B kann nicht auf die Präsupposition bezogen werden.

A: Der König von Frankreich ist kahlköpfig.

B: Nein, das stimmt nicht. \#Frankreich hat keinen König.

B': Hey, warte mal! Frankreich hat doch gar keinen König!

Wie mit den Sätzen der P-Familie kann jedoch auch mit der Hey, wait a minute!-Strategie nicht eindeutig ermittelt werden, ob es sich bei einer Inferenz um eine Präsupposition handelt, da auch andere [-at-issue]-Inferenzen mit Hey, wait a minute! erfragt werden können, wie u.a. Potts (2011) und Roberts et al. (2009) beobachten. Beispielsweise können auch appositive Relativsätze nur indirekt angefochten werden, wie folgendes Beispiel zeigt.

A: Jap, der gerade in Indonesien ist, fährt gerne ans Meer.

B: Nein, das stimmt nicht. \#Jap ist gerade in China.

B': Hey, warte mal! Jap ist doch gerade in China!

\footnotetext{
${ }^{24}$ Natürlich gibt es diverse Möglichkeiten, einen indirekten Widerspruch zu formulieren, von denen Hey, warte mal! nur eine darstellt.
} 


\section{Typische Eigenschaften der Nebenstruktur}

Dass appositive Relativsätze aber nicht präsupponiert sind, wurde bereits in Abschnitt 3.1 gezeigt (vgl. Beispiel (96) sowie Holler 2005, 54 und Roberts et al. 2009). Dasselbe gilt für Expressiva, die ebenfalls keine bereits im Common Ground gegebene Information ausdrücken, und demnach nicht präsupponiert sind (vgl. Beispiel (98) in Abschnitt 3.1), aber dennoch nur indirekt negiert werden können. So kann sich B's Widerspruch in dem folgenden Beispiel nur auf die Proposition Tom ist heute mit Kochen dran beziehen, nicht aber auf den expressiven Ausdruck. Aus diesem Grund ist eine Weiterführung mit Tom ist sehr nett nicht möglich. Ganz anders liegt der Fall bei der Äußerung von B', wo eine Weiterführung problemlos möglich ist.

Dieser Idiot Tom ist heute mit Kochen dran.

B: Nein, das stimmt nicht. \#Tom ist doch eigentlich ganz nett.

B': Hey, warte mal! Tom ist doch eigentlich ganz nett.

Dass mit der hier vorgestellten Form des Kommentartests keine Präsuppositionen, sondern die kommunikative Relevanz des Gesagten ermittelt wird, zeigt bereits Posner (1972). Eine empirische Überprüfung dieser Ansicht findet sich bei Potts (2008), dessen Korpusuntersuchung zur Verwendung von Wait a minute! in Abschnitt 3.2.2.1 vorgestellt wird. Aber bereits an dieser Stelle können wir sehen, dass mit appositiven Relativsätzen und Expressiva eindeutig nicht-präsupponierte Inhalte angefochten werden und Hey, wait-a-minute! somit wie die im vorherigen Abschnitt diskutierten Projektionstests nicht zur Ermittlung von Präsuppositionen geeignet ist. Stattdessen argumentieren Posner (1972) und Roberts et al. (2009), dass mithilfe von Widerspruchsstrategien Bedeutungsaspekte, die nicht Teil der Hauptstruktur einer Äußerung sind, ermittelt werden. Genau wie semantische Operatoren wie Negation oder der Fragesatzoperator in den Projektionstests (vgl. (91)) bezieht sich auch ein direkter Widerspruch des Hörers nur auf Äußerungsteile der Hauptstruktur, weswegen [-at-issue]-Inhalte davon unbetroffen bleiben.

Allerdings können auch [+at-issue]-Inhalte mithilfe von Hey, wait-a-minute! aufgegriffen werden wie in dem folgenden Beispiel (vgl. dazu auch die Ergebnisse der Korpusuntersuchung in Potts 2008 in Abschnitt 3.2.2.1).

A: Peter wohnt jetzt wieder bei seinen Eltern.

B: Hey, warte mal! Peter wohnt doch nicht bei seinen Eltern! Er ist doch in eine WG gezogen!

B' Nein, das stimmt nicht. Er ist in eine WG gezogen.

Der entscheidende Unterschied zwischen [+at-issue]- und [-at-issue]-Inhalten besteht nun aber darin, dass Letzteren nur indirekt und nicht direkt widersprochen werden kann, wohingegen [+at-issue]-Inhalte beide Formen des Widerspruchs erlauben.

Auf der Grundlage von Roberts et al. (2009)'s und Potts (2011)'s Beobachtung, derzufolge [-at-issue]-Inhalte nur indirekt anfechtbar sind, ist zu erwarten, dass alle Nebensatztypen aus Gruppe 2 von einem direkten Widerspruch unbeeinflusst blei- 
ben. Diese Hypothese wird in Abschnitt 3.2.3 mithilfe einer empirischen Pilotstudio überprüft. Zuvor noch wird ein kurzer Überblick über die wichtigsten bisherigen empirischen Untersuchungen zur Unterscheidung zwischen [+at-issue]- und [-atissue]-Inhalten geliefert.

\subsubsection{Bisherige Untersuchungen}

\subsubsection{Potts (2008)}

Potts (2008) untersucht in einer Korpusstudie gesprochensprachlicher Daten, welche Inhalte in der natürlichen Sprache mit der Diskursstrategie Wait-a-minute erfragt werden. Der Studie liegt die oben erwähnte Beobachtung zugrunde, dass Wait-aminute häufig als Präsuppositionstest verwendet wird, ohne dass eine empirische Überprüfung der damit verbundenen Voraussetzung (123) vorliegt.

If Wait a minute is a suitable method for objecting to a meaning $M$, then $M$ is expressed as a presupposition.

Somit handelt es sich bei Potts (2008) nicht um eine Studie zur Erforschung der Unterscheidung von [+at-issue]- und [-at-issue]-Inhalten. Dennoch sind die Ergebnisse auch für die Erforschung von Haupt- und Nebenstruktur eines Diskurses von Bedeutung, weswegen sie kurz vorgestellt werden.

Der in Potts (2008) untersuchte Korpus umfasst 439 annotierte, äußerungsinitiale Belege von Wait-a-minute, allesamt aus Interviewscripten von CNN. Für jedes Beispiel wurden jeweils sechs Zeilen vor und sechs Zeilen nach Wait-a-minute als Kontext erfasst, um auf dieser Grundlage zu bestimmen, auf welche inhaltlichen Aspekte mit Wait-a-minute Bezug genommen wird. Dennoch blieb bei insgesamt 103 Belegen unklar, worauf sich der Widerspruch bezieht. Für die übrigen 336 Belege unterscheidet Potts (2008) vier Arten von Bedeutungsaspekten, die mittels Waita-minute hinterfragt werden. In insgesamt 129 Belegen nimmt der Sprecher mit der Diskursstrategie Bezug auf Präsuppositionen und Implikaturen, wobei eine Darstellung, wie sich die Zahl auf die beiden Typen von Inferenzen verteilt, nicht geliefert wird. Fälle, in denen [+at-issue]-Inhalte angefochten werden, sind mit 122 Belegen vertreten. In weiteren 67 Fällen bezieht sich Wait-a-minute auf Diskursbedingungen, beispielsweise, wenn $\mathrm{S}$ die Unterhaltung unterbrechen möchte. In den übrigen 38 Belegen antwortet S mit Wait-a-minute auf Äußerungen, die S nicht für angemessen oder richtig hält.

Die Ergebnisse dieser ersten Studie zur Verwendung von Wait-a-minute zeigen, dass die in (123) abgebildete Annahme nicht zutreffend ist, da neben Präsuppositionen auch zahlreiche weitere Bedeutungsaspekte mittels Wait-a-minute hinterfragt werden können. Dazu gehören andere [-at-issue]-Inhalte, aber auch Bedeutungen, die [+at-issue] sind. Damit liegt ein empirischer Nachweis für die Unzulänglichkeit von Wait-a-minute als Präsuppositionstest vor. Offen bleibt jedoch die Frage, ob Präsuppositionen und anderen [-at-issue]-Inhalten nur indirekt widersprochen werden kann, und sie sich somit von Teilen der Hauptstruktur unterscheiden, auf 


\section{Typische Eigenschaften der Nebenstruktur}

welche auch mit einem direkten Widerspruch Bezug genommen werden kann. Auch hierzu liegen bislang nur sehr wenige empirische Studien vor, von denen zwei in den folgenden beiden Abschnitten kurz vorgestellt werden.

\subsubsection{Amaral et al. (2011)}

Amaral et al. (2011) gehen der Frage nach, ob Sprecher für die Unterscheidung zwischen [+at-issue]- und [-at-issue]-Inhalten sensibel sind, und ob diese Unterscheidung bei der Sprachverarbeitung eine Rolle spielt. Zu diesem Zweck überprüfen sie anhand eines Lesezeit- und Bewertungsexperiments das Zutreffen der in Abschnitt 3.2.1 genannten (und bisher rein introspektiven) Beobachtung, derzufolge nur [+at-issue]-Inhalten direkt widersprochen werden kann. Dem Experimentaufbau liegt die folgende Überlegung zugrunde: In Entscheidungsfragen wird die entsprechende Alternativenmenge, also die Bipartition $\{\mathrm{p}, \neg \mathrm{p}\}$, durch den [+at-issue]-Inhalt der Äußerung eröffnet und nicht durch Äußerungsteile der Nebenstruktur. Dies zeigt sich an folgendem Beispiel, in dem die von Q eröffnete Alternativenmenge durch den assertierten Inhalt des Satzes bestimmt wird und demnach \{Marche fährt mit Homers Auto, $\neg$ Marche fährt mit Homers Auto\} lautet. Die Präsupposition des Satzes, derzufolge Homer ein Auto besitzt, eröffnet dagegen keine Menge an Alternativen. Aus diesem Grund bildet nur B, nicht aber B', eine wohlgeformte Antwort auf Q.

$$
\begin{aligned}
& \text { Q: Fährt Marche mit Homers Auto? } \\
& \text { B: Ja, sie darf sein Auto benutzen. } \\
& \text { B':\#Ja, Homer hat ein Auto. }
\end{aligned}
$$

Während die von Q eröffnete Alternativenmenge durch B auf ein Element reduziert wird, B bildet demnach eine vollständige Antwort, da $\#(\Pi / A)=1$, führt B' zu keiner Reduktion der Alternativenmenge. Es gilt demnach $\#(\Pi / A)=n$ und $B^{\prime}$ ist als Antwort auf Q laut Definition (17) (vgl. Abschnitt 2.1.1) irrelevant. Daraus folgt, dass auch die entsprechende negative Antwort $\mathrm{C}$ irrelevant und damit als Antwort unangemessen ist. Soll die Präsupposition angefochten werden, muss eine indirekte Form des Widerspruchs wie C' gewählt werden.

$$
\begin{aligned}
& \text { Q: Fährt Marche mit Homers Auto? } \\
& \text { C: \#Nein, Homer hat kein Auto. } \\
& \text { C': Hey, warte mal! Homer hat doch gar kein Auto. }
\end{aligned}
$$

Amaral et al. (2011) untersuchen nun für vier verschiedene [-at-issue]-Inferenzen, ob diese Beobachtung generalisierbar ist, d.h., ob Teile der Nebenstruktur tatsächlich nur indirekt anfechtbar sind. Dabei handelt es sich um die durch again, stop, only und better than ausgelösten Inferenzen. In der Studie werden demnach nur Präsuppositionen als Beispiel für [-at-issue]-Inhalte getestet (zum Unterschied zwischen Präsuppositionen und anderen, nicht-präsupponierten Teilen der Nebenstruktur vgl. Abschnitt 4). Ein weiterer Punkt, dem Amaral et al. (2011) in ihrem Experiment nachgehen, betrifft die Frage, ob sich alle vier untersuchten Präsuppositionsauslöser 
gleich verhalten oder aber, ob das Konzept der (Nicht-)At-issueness graduell ist und Inhalte der Nebenstruktur demnach unterschiedlich stark dazu geeignet sind wiederaufgegriffen zu werden.

Die Probanden, 24 Muttersprachler des Englischen, wurden mit Frage-AntwortPaaren konfrontiert und aufgefordert, die jeweilige Antwort auf einer Skala von eins bis fünf danach zu beurteilen, wie natürlich sie ihnen in diesem Kontext erscheint. Bei den Fragen handelte es sich ausschließlich um Entscheidungsfragen, die jeweils einen der vier getesteten Präsuppositionsauslöser enthielten. Die in allen Fällen negative Antwort wurde jeweils mit einem direkten Widerspruch $N_{o}, \ldots$ eingeleitet. Die Weiterführung der Antwort folgte nun jeweils einer von zwei möglichen Bedingungen:

Bedingung 1: Die Weiterführung bezieht sich auf den [+at-issue]-Inhalt der Frage. Bedingung 2: Die Weiterführung bezieht sich auf den [-at-issue]-Inhalt der Frage.

Dies ist in den folgenden Beispielen nach Amaral et al. (2011) veranschaulicht:

\section{Bedingung 1:}

Q: Did Julia stop smoking?

A: No, she smokes.

\section{Bedingung 2:}

Q: Did Julia stop smoking?

A': No, she didn't use to smoke.

Das Experiment wurde in E-Prime durchgeführt, wobei auch die Antwortzeit mitgemessen wurde. Wenn Sprecher für die Unterscheidung zwischen Haupt- und Nebenstruktur sensibel sind, ist zu erwarten, dass die Sätze der Bedingung 2 systematisch schlechter bewertet werden als Bedingung 1. Zudem sollte das Abrufen von Hintergrundinformationen nach Amaral et al. (2011) mehr Zeit in Anspruch nehmen als die Verarbeitung der Hauptstruktur, so dass Amaral et al. (2011) für Bedingung 2 auch mit längeren Reaktionszeiten rechneten.

Wie erwartet, bevorzugten die Probanden die Sätze der Bedingung 1 signifikant vor denen von Bedingung 2. Zudem erzielten die Testsätze von Bedingung 1 systematisch niedrigere Reaktionszeiten. Ein weiterer Punkt, der durch die Ergebnisse suggeriert wird, ist, dass [-at-issue]-Inhalte oder zumindest Präsuppositionen, ein in sich heterogenes Phänomen darstellen, da die vier Präsuppositionsauslöser unterschiedliche Bewertungen erzielt haben. Dies überprüfen Amaral et al. (2011) in einer Folgestudie, welche ebenfalls bestätigt, dass Präsuppositionen je nach Auslöser unterschiedlich stark zur Nebenstruktur gezählt werden. Damit trägt die Studie zu einer experimentell fundierten Typologie von Präsuppositionsauslösern bei. ${ }^{25}$ Die Studie von Amaral et al. (2011) liefert einen wichtigen Beitrag zur Erforschung

\footnotetext{
${ }^{25}$ Da Präsuppositionen nicht im Zentrum dieser Arbeit stehen, wird auf die Folgestudie nicht weiter
} eingegangen. Für Details vgl. Amaral et al. (2011). 


\section{Typische Eigenschaften der Nebenstruktur}

von Haupt- und Nebenstruktur. Allerdings besitzt sie noch stark den Charakter einer ersten Pilotstudie, insbesondere aufgrund der geringen Probandenzahl. Zudem werden als Beispiele für [-at-issue]-Inhalte ausschließlich Präsuppositionen getestet und keine anderen [-at-issue]-Aspekte wie beispielsweise appositive Relativsätze oder Expressiva. Ein Kritikpunkt, den Amaral et al. (2011) selbst nennen, bezieht sich auf die Tatsache, dass keine der beiden Antworten A und A' vollkommen natürlich klingt, insofern als dass die gesamte Proposition wiederholt wird. Da dies aber beide Bedingungen betrifft, sollte dieser Effekt nur eine geringe oder gar keine Rolle spielen. Zudem kann man kritisieren, dass das Experimentdesign bezogen auf die beiden Bedingungen nicht objektiv ist. Durch den Kontext, d.h. den jeweiligen Fragesatz, wird bereits vorgegeben, dass der [+at-issue]-Inhalt der Äußerung erfragt wird und nicht die Präsupposition. Dies wird daran deutlich, dass die jeweilige Entscheidungsfrage durch Voranstellung des Matrixprädikats gebildet wird, die Antwort aber das Prädikat für die Nebenstruktur verwendet. Dies bedeutet aber, dass durch das Experimentdesign bereits eine der zwei Bedingungen, nämlich Bedingung 1, bevorzugt wird. Aus diesem Grund ist es fraglich, ob durch die Studie tatsächlich gezeigt wird, dass nur [+at-issue]-Inhalten direkt widersprochen werden kann.

\subsubsection{Xue/Onea (2011)}

Xue/Onea (2011) beschreiben zwei Experimente, in denen ebenfalls verschiedene Präsuppositionsauslöser in Bezug auf ihr Projektionsverhalten und bezogen auf die Frage, ob der durch sie ausgelösten Inferenz direkt widersprochen werden kann, untersucht werden. Sie übernehmen dabei die Annahme von Roberts et al. (2009) und Simons et al. (2011), derzufolge das Konzept der Nicht-At-issueness mit einer hohen Projektionswahrscheinlichkeit korreliert. In einem ersten Experiment zeigen Xue/Onea (2011), dass die Wahrscheinlichkeit, dass eine Inferenz unter Einbettung projiziert, je nach Präsuppositionsauslöser unterschiedlich hoch ist. In einem Folgeexperiment überprüfen sie dann für die gleichen Auslöser, ob eine hohe Projektionswahrscheinlichkeit damit korreliert, dass dieser Inferenz nur indirekt widersprochen werden kann, was die Autoren ebenfalls in Anlehnung an Roberts et al. (2009) als distinktives Zeichen für Nicht-At-issueness deuten.

Da das erste Experiment das Projektionsverhalten von unterschiedlichen Inferenzen testet und nicht die auf sie angewandten Widerspruchsstrategien, soll es an dieser Stelle nur kurz zusammengefasst werden (für weitere Details vgl. Xue/Onea 2011). Grundlegend ist die Beobachtung, dass Präsuppositionen je nach Kontext nicht immer projizieren. So überlebt die Inferenz, derzufolge Peter die Angewohnheit hatte zu rauchen, in dem folgenden Beispiel nicht: ${ }^{26}$

\footnotetext{
${ }^{26}$ Allerdings ist umstritten, ob es sich in einem solchen Fall überhaupt um eine Präsupposition handelt. Xue/Onea (2011) verfolgen hier einen lexikalischen Präsuppositionsansatz, demzufolge eine durch einen klassischen Präsuppositionsauslöser wie stop ausgelöste Inferenz immer präsupponiert ist. Im Rahmen einer eher pragmatischen Präsuppositionstheorie dagegen wird angenommen, dass Präsuppositionen wie konversationelle Implikaturen nur in bestimmten Kontexten ausgelöst werden, in Beispiel (128) demnach gar keine Präsupposition vorliegt (vgl.
} 
(128) Peter didn't stop smoking. He never smoked!

Nach Roberts et al. (2009) und Simons et al. (2011) projizieren Präsuppositionen deswegen, weil sie in der Regel [-at-issue] sind (vgl. Abschnitt 3.1, sowie Kapitel 4 zur Einordnung von Präsuppositionen). Umgekehrt folgt daraus, dass Präsuppositionen, die wie in Beispiel (128) nicht projizieren, Teil des [+at-issue]-Inhalts einer Äußerung sind. In ihrem WebExp-basiertem Experiment testen Xue/Onea (2011) nun die Präsuppositionsauslöser wissen, erfahren, auch und wieder daraufhin, ob die durch sie ausgelöste Präsupposition bei Einbettung in ein Konditionalsatzgefüge überlebt. 34 deutsche Muttersprachler wurden aufgefordert, für Satzpaare wie das folgende die jeweilige Frage (hier (129-b)) zu beantworten. Den Kontext bildet jeweils ein Konditionalsatzgefüge (hier (129-a)), in dessen Antezedens einer der vier untersuchten Präsuppositionsauslöser eingebettet ist. Die Frage zielt dann jeweils auf die Negation dieser Präsupposition ab.

(129) a. Wenn Paul weiß, dass Christine gerne Tee trinkt, schenkt er ihr eine Teekanne.

b. Ist es möglich, dass Christine nicht gerne Tee trinkt?

Zur Beantwortung der jeweiligen Fragen mussten die Probanden eine der folgenden drei Antwortmöglichkeiten auswählen:
a. Ja, das ist möglich.
b. Nein, das ist nicht möglich.
c. Ich weiß es nicht.

Die Wahrscheinlichkeit, mit der die Probanden bei einem Präsuppositionsauslöser die Nein-Antwort wählen, entspricht nach Xue/Onea (2011) der Projektionswahrscheinlichkeit für diese spezifische Präsupposition. Dagegen wird ein Proband die durch $J a$ eingeleitete Antwort wählen, wenn er sich einen Interpretationskontext rekonstruiert, in dem die in das Konditionalgefüge eingebettete Präsupposition nicht projiziert. Mit ihrer Studie erzielten Xue/Onea (2011) die in Tabelle 3.2 dargestellten Ergebnisse. Daraus wird ersichtlich, dass die Wahrscheinlichkeit, mit der eine präsupponierte Inferenz unter Einbettung projiziert, wesentlich vom jeweiligen Präsuppositionsauslöser abhängt. Inferenzen, die durch ein semifaktives Verb eingebettet sind, projizieren mit einer weitaus geringeren Wahrscheinlichkeit als die durch lexikalische Trigger wie auch und wieder ausgelösten Präsuppositionen.

\begin{tabular}{|c|c|c|c|c|}
\hline & wissen & erfahren & auch & wieder \\
\hline Nein,... (Projektion) & 38,24 & 51,96 & 87,25 & 99,02 \\
\hline
\end{tabular}

Tabelle 3.2.: Ergebnisse, Experiment 1 aus Xue/Onea (2011)

In einem zweiten Schritt überprüfen Xue/Onea (2011), ob die unterschiedlichen Projektionswahrscheinlichkeiten der vier Präsuppositionen mit einem unterschiedlichen

beispielsweise Geurts 1999 und Stalnaker 1974). 


\section{Typische Eigenschaften der Nebenstruktur}

Grad an (Nicht-)At-issueness korrelieren. Hierzu nutzen sie die Tatsache, dass nur [+at-issue]-Inhalten direkt widersprochen werden kann (vgl. Simons et al. 2011 und Abschnitt 3.2.1). 29 Probanden nahmen an dem WebExp-basiertem Experiment teil, bei dem sie mit einem Satz, der einen der vier untersuchten Präsuppositionsauslöser enthielt, konfrontiert wurden. Anschließend wurden sie gebeten, aus drei möglichen Antworten diejenige auszuwählen, die ihrer Meinung nach am besten passt. In allen drei vorgegebenen Antworten wird der Präsupposition des initialen Satzes widersprochen, die vorgegebenen Antworten unterscheiden sich jedoch im Grad ihrer Direktheit. Mit Nein wird der Präsupposition direkt widersprochen, dagegen bildet $J a$, und eine indirekte Form des Widerspruchs. Ja, aber befindet sich, was die Direktheit betrifft, zwischen diesen beiden Antworten. Dies ist an dem folgenden Beispiel aus Xue/Onea (2011) illustriert:

(131) Tina hat gerade erfahren, dass Max im Urlaub ist.

a. Ja, und Max ist gar nicht im Urlaub.

b. Ja, aber Max ist gar nicht im Urlaub.

c. Nein, Max ist gar nicht im Urlaub.

Nach Xue/Onea (2011) ist zu erwarten, dass Nein nur dann als Antwort gewählt wird, wenn der Proband die Präsupposition zum [+at-issue]-Inhalt der initialen Äußerung zählt. Da die Testsätze ohne weiteren Kontext gestellt werden, gehen die Autoren davon aus, dass die Probanden unbewusst einen Interpretationskontext mit einer geeigneten Q rekonstruieren. Wählen Sie nun die mit Nein eingeleitete Antwort, ist dies ein Indiz dafür, dass die Präsupposition in dem jeweils rekonstruierten Kontext Teil der Hauptstruktur ist. Je mehr Probanden bezogen auf einen Testsatz die Nein-Antwort wählen, umso höher ist demnach die Wahrscheinlichkeit, dass die entsprechende Präsupposition zum [+at-issue]-Inhalt der initialen Äußerung gezählt wird. Die für die vier unterschiedlichen Präsuppositionsauslöser erzielten Ergebnisse sind in Tabelle 3.3 abgebildet.

\begin{tabular}{|c|c|c|c|c|}
\hline & wissen & erfahren & auch & wieder \\
\hline Nein,... ([+at-issue $])$ & 72,41 & 50,00 & 25,86 & 22,41 \\
\hline
\end{tabular}

Tabelle 3.3.: Ergebnisse, Experiment 2 aus Xue/Onea (2011)

Wir können sehen, dass die durch die semifaktiven Verben wissen und erfahren ausgelösten Präsuppositionen von den Probanden mit einer größeren Wahrscheinlichkeit als Teil des [+at-issue]-Inhalts analysiert wurden als die durch auch und wieder ausgelösten Inferenzen. Für letztere ist es demnach deutlich schwieriger, einen Kontext zu rekonstruieren, in dem die Präsupposition der Beantwortung einer Frage Q dient. Vergleicht man die beiden Tabellen, lässt sich eine eindeutige Korrelation zwischen dem Projektionsverhalten der vier getesteten Präsuppositionen und der Frage, ob ihnen direkt widersprochen werden kann, feststellen. Die Präsuppositionen, die mit einer hohen Wahrscheinlichkeit unter Einbettung heraus projizieren, lassen umgekehrt nur mit einer geringen Wahrscheinlichkeit einen direkten Widerspruch zu, was 
als Indiz für ihre Zugehörigkeit zur Nebenstruktur gewertet wird. Umgekehrt wird den Präsuppositionen, welche nur mit einer geringen Wahrscheinlichkeit projizieren, vermehrt direkt widersprochen, was Xue/Onea (2011) als Zeichen dafür werten, dass sie von den Probanden bevorzugt als [+at-issue]-Inhalte analysiert werden. Damit stehen die Ergebnisse im Einklang mit der in u.a. Roberts et al. (2009) vertretenen Hypothese, dass Projektion mit dem Konzept der Nicht-At-issueness zu erklären ist. Allerdings ist zu bedenken, dass die hohe Anzahl an Nein-Antworten bei den Präsuppositionsauslösern wissen und erfahren auch darauf zurückgeführt werden könnte, dass die beiden mit $J a$ eingeleiteten Antworten in diesem Kontext nur bedingt sinnvoll sind. Wird mit einer indirekten Negation wie in dem folgenden Beispiel durch Sprecher B nur die Präsupposition, nicht aber die Assertion, abgelehnt, ist die resultierende Lesart nicht schlüssig, da unklar ist, wie Petra etwas wissen kann, das nicht zutreffend ist. Die Äußerung ist als Antwort auf A nur dann schlüssig, wenn das Verb wissen nicht-faktiv im Sinne von 'glauben' oder 'erzählen' interpretiert wird. Da die Rekonstruktion dieser Interpretation aber ein höheres Maß an Aufwand benötigt, könnte dies dazu führen, dass die Probanden die mit Nein eingeleitete Antwort bevorzugen.

A: Petra weiß, dass Hans die Kekse gestohlen hat.

B: Ja, und Hans hat sie nicht gestohlen.

Anders sieht es aus, wenn die durch auch ausgelöste Präsupposition indirekt negiert wird. Hier ist die Lesart, derzufolge Sprecher B zwar die Präsupposition ablehnt, das Zutreffen der Assertion aber bejaht, durchaus sinnvoll.

A: Peter hat auch Eva einen Blumenstrauß geschenkt.

B: Ja, aber er hat sonst niemandem einen geschenkt.

Prinzipiell ist die Beobachtung von Xue/Onea (2011), derzufolge semifaktive Komplementsätze ohne ihren Bezugssatz [+at-issue]-Inhalt ausdrücken können, zutreffend, wie bereits in Abschnitt 2.2.2 gezeigt wurde (vgl. die Beispiele (52) und (53)). Ob die [+at-issue]-Lesart für semifaktive Komplementsätze jedoch mit einer so hohen Wahrscheinlichkeit gewählt wird, wie sie Xue/Onea (2011) feststellen (72\% für Objektsätze des Verbs wissen), ist zweifelhaft und möglicherweise auch auf Schwächen im Testdesign zurückzuführen.

\subsubsection{Zusammenfassung}

In diesem Abschnitt wurden bisherige Untersuchungen zu direkten und indirekten Widerspruchsstrategien, sowie deren Verhältnis zum Konzept der At-issueness diskutiert. Potts (2008) testet in seiner Korpusstudie, welchen inhaltlichen Aspekten mit der indirekten Negation Wait-a-minute widersprochen werden kann, und kommt zu dem Schluss, dass es sich um diverse Inhalte handelt, [+at-issue]-Bedeutungen inbegriffen. Damit hat er gezeigt, dass Wait-a-minute kein geeigneter Präsuppositionstest ist. Offen bleibt jedoch die Frage, ob Teilen der Nebenstruktur nur indi- 


\section{Typische Eigenschaften der Nebenstruktur}

rekt widersprochen werden kann. Amaral et al. (2011) versuchen hier anzuknüpfen, indem sie zeigen, dass Fälle, in denen sich eine direkte Negation auf den [-atissue]-Inhalt einer Äußerung bezieht, von Muttersprachlern als nicht-wohlgeformt empfunden werden. Problematisch an ihrer experimentellen Studie ist jedoch, dass der jeweilige Kontext, welcher in Form einer Entscheidungsfrage vorgegeben wird, bereits eine Weiterführung des [+at-issue]-Inhalts nahelegt. Damit wird in der Studie nicht getestet, ob der Nebenstruktur direkt widersprochen werden kann. Stattdessen wird vielmehr gezeigt, dass die Probanden sensibel für die Unterscheidung zwischen Haupt- und Nebenstruktur sind und aufgrund der entsprechenden grammatischen Markierungen erkennen, dass nach dem [+at-issue]-Inhalt der Äußerung gefragt wird.

Auch Xue/Onea (2011) testen unterschiedliche Widerspruchsstrategien im Zusammenhang mit der diskursstrukturellen Unterscheidung in Haupt- und Nebenstruktur. Anders als bei Amaral et al. (2011) geben Xue/Onea (2011) den Kontext jedoch nicht als Entscheidungsfrage, sondern als Deklarativsatz vor, wodurch keine Antworterwartung transportiert wird. Ihre Ergebnisse belegen, dass ein Zusammenhang zwischen dem diskursstrukturellen Konzept der At-issueness und dem Projektionsverhalten von Inferenzen besteht. Zudem stellen Xue/Onea (2011) fest, dass es sich bei der Zugehörigkeit zur Haupt- bzw. zur Nebenstruktur um ein graduelles Phänomen handelt. Für die folgenden Abschnitte ist die Untersuchung von Xue/Onea (2011) aber aus einem anderen Grund von besonderem Interesse. So wird in ihrem zweiten Experiment die Beobachtung, dass nur [+at-issue]-Inhalten direkt widersprochen werden kann, als Test zur Ermittlung von [+at-issue]-Inhalten verwendet. Da Xue/Onea (2011) hierbei eine eindeutige Korrelation zwischen Nicht-At-issueness und Projektion feststellen, können wir davon ausgehen, dass die verwendete Testmethode geeignet ist, um zwischen inhaltlichen Aspekten der Haupt- und solchen der Nebenstruktur zu unterscheiden. Diese Annahme ist grundlegend für die Untersuchung im folgenden Abschnitt, in deren Zentrum unterschiedliche Nebensatztypen stehen.

In den bisherigen empirischen Studien zur Verwendung von direkten und indirekten Widerspruchsstrategien wurden ausschließlich Präsuppositionsauslöser getestet. Im folgenden Abschnitt wird nun eine Fragebogenuntersuchung vorgestellt, in der die insgesamt acht Nebensatztypen der Gruppen 1 und 2 miteinander verglichen werden und mit appositiven Relativsätzen somit auch Sätze untersucht werden, die eindeutig nicht präsupponiert sind. Im Zentrum steht dabei die Frage, ob sich die unterschiedlichen Nebensätze darin unterscheiden, wie sensibel sie für direkte Widerspruchsstrategien sind.

\subsubsection{Fragebogenstudie}

\subsubsection{Aufbau und Durchführung}

In Abschnitt 2.2 wurde dafür argumentiert, dass zwei unterschiedliche Gruppen von Nebensatztypen zu unterscheiden sind (vgl. (86)). Die Nebensatztypen der Gruppe 
1 können dazu verwendet werden, die jeweils aktuelle $\mathrm{Q}$ zu beantworten, in diesem Fall projizieren sie nicht aus dem Skopus eines inferenztilgenden Operators heraus, wie in Abschnitt 3.1.2 gezeigt wurde. Dazu zählen Komplementsätze von Brückenund semifaktiven Verben, weil-Sätze und restriktive Relativsätze mit einem indefiniten Antezedens. Da sich direkte Widerspruchsstrategien auf den [+at-issue]-Inhalt einer Äußerung beziehen, ist zu erwarten, dass diesen Nebensatztypen direkt widersprochen werden kann. Demgegenüber stehen die Nebensatztypen der Gruppe 2, zu der Komplementsätze von faktiven Verben, temporale Adverbialsätze, appositive Relativsätze und restriktive Relativsätze mit einem definiten Antezedens zählen. In Abschnitt 2.2 haben wir gesehen, dass diese Nebensatztypen nicht dazu verwendet werden können, die jeweilige Q zu beantworten, zudem projizieren sie immer unter Einbettung (vgl. Abschnitt 3.1.2). Demnach ist zu erwarten, dass dem Inhalt eines Nebensatzes aus Gruppe 2 nur indirekt widersprochen werden kann, da er als Teil der Nebenstruktur nicht sensibel für direkte Negation ist. Beide Annahmen wurden mithilfe einer Fragebogenstudie überprüft, wobei immer Satzpaare bestehend aus einer Assertion A und einer dazugehörigen Antwort B vorgegeben wurden. ${ }^{27}$ Jede Assertion A enthielt einen Nebensatz, die Antwort B lautete für jedes Testpaar gleich, nämlich einfach Nein. Mit B liegt somit eine direkte Form des Widerspruchs vor. Ein Beispiel hierfür bildet (134).

A: Petra war einkaufen, bevor sie die Kinder abgeholt hat.

B: Nein.

In den Instruktionen wurden die Versuchsteilnehmern gebeten, von der Annahme auszugehen, dass B mit seinem Widerspruch immer recht hat, um sicher zu gehen, dass die Negation Nein auch auf Äußerung A bezogen wird. Auf dieses Satzpaar folgte eine fettgedruckte Entscheidungsfrage, in welcher jeweils das Zutreffen der in A eingebetteten Proposition zur Debatte stand. Beispielsweise folgte auf das Satzpaar in (134) die Frage Hat Petra die Kinder abgeholt?. Die fettgedruckte Frage eröffnet eine Alternativenmenge $\{\mathrm{q}, \neg \mathrm{q}\}$, wobei q den propositionalen Inhalt des Nebensatzes, also in diesem Fall Petra hat die Kinder abgeholt, bezeichnet. Die teilnehmenden Versuchspersonen wurden nun aufgefordert, die fettgedruckte Frage mithilfe einer vorgegebenen Skala von 1 (= sehr sicher, dass nein) bis 7 (= sehr sicher, dass ja) zu beantworten. Die Antwort 7 entspricht dabei dem Zutreffen von q, 1 sagt aus, dass $\neg q$ zutrifft und mit der Wahl der Antwortmöglichkeit 4 legt sich ein Proband auf keine der beiden Alternativen fest. Auf diese Weise lässt sich testen, ob die Probanden die direkte Negation auf den Nebensatzinhalt beziehen und somit, ob sie

\footnotetext{
${ }^{27}$ Es wäre natürlich auch wünschenswert, das in Abschnitt 3.1 rein introspektiv beurteilte Projektionsverhalten der abhängigen Sätze empirisch zu überprüfen. Widerspruch und Projektion beziehen sich allerdings auf sehr ähnliche Eigenschaften von [-at-issue]-Inferenzen, nämlich dass diese nicht im Skopus von semantischen Operatoren stehen. In beiden Fällen wird ein Negationsoder ein anderer Operator von außerhalb auf den Nebensatz bezogen, was nur erfolgt, wenn der Nebensatz ohne seinen Bezugssatz [+at-issue]-Inhalt ausdrückt. Die Korrelation zwischen Projektionswahrscheinlichkeit und Sensibilität für einen direkten Widerspruch wurde zudem bereits empirisch von Xue/Onea (2011) nachgewiesen.
} 
den Nebensatz als Teil der Hauptstruktur interpretieren. Gleichzeitig wird der Grad der Sicherheit, mit der ein Versuchsteilnehmer antwortet, kontrolliert. Im Folgenden sind einige Beispiele dargestellt, eine vollständige Auflistung aller Testsätze befinden sich im Anhang.

A: Andreas hat erzählt, dass die Schule renoviert wird.

B: Nein.

Wird die Schule renoviert?

\begin{tabular}{|lllllllll|}
\hline (sehr sicher, dass nein) & 1 & 2 & 3 & 4 & 5 & 6 & 7 & (sehr sicher, dass ja) \\
\hline
\end{tabular}

A: Nadine verkauft ihr Auto, weil es kaputt ist.

B: Nein.

Ist Nadines Auto kaputt?

(137) A: Maria bereut, dass sie Jonas verlassen hat.

B: Nein.

Hat Maria Jonas verlassen?

(138) A: Paul, der Frank verprügelt hat, wohnt in Göttingen.

B: Nein.

Wurde Frank von Paul verprügelt?

Da B's Antwort einen direkten Widerspruch darstellt, sollte sich dieser nach Roberts et al. (2009), Simons et al. (2011) und Xue/Onea (2011) nur auf den [+at-issue]Inhalt der Äußerung A beziehen. Ob der Inhalt des in A eingebetteten Nebensatzes ebenfalls von B's negativer Antwort betroffen ist, hängt demnach davon ab, ob der entsprechende Nebensatz zur Haupt- oder Nebenstruktur von A gehört. In den Beispielen (137) und (138) haben wir es mit Nebensatztypen der Gruppe 2 zu tun. Demnach sollte sich die direkte Negation von B nicht auf den jeweiligen Inhalt des Nebensatzes beziehen, das Zutreffen der Nebensatzproposition q wird durch B also nicht hinterfragt. Aus diesem Grund ist zu erwarten, dass die Probanden in Beispielen mit Nebensatztypen der Gruppe 2 auf der Skala einen hohen Wert wählen. Dagegen liegen in den Testsätzen (135) und (136) Nebensatztypen der Gruppe 1 vor, welche je nach Kontext [+at-issue]- oder [-at-issue]-Inhalt ausdrücken können. In Abhängigkeit davon, wie der jeweilige Nebensatz interpretiert wird, kann sich B's direkte Negation auch auf den Nebensatzinhalt beziehen. Da die Sätze ohne weiteren Kontext präsentiert werden, ist anzunehmen, dass sich die Versuchsteilnehmer einen geeigneten Interpretationskontext in Form einer zu beantwortenden impliziten Q rekonstruieren (vgl. zu dieser Annahme auch Xue/Onea 2011). Vor diesem Hintergrund entscheiden die Probanden dann, ob der Nebensatzinhalt [+at-issue] ist oder nicht. Interpretiert ein Proband den Nebensatz nun als [+at-issue], bezieht sich B's direkter Widerspruch auch auf den Inhalt des eingebetteten Satzes. In diesem Fall ist zu erwarten, dass ein niedriger Wert gewählt wird, da das Nicht-Zutreffen des Nebensatzinhalts (also $\neg \mathrm{q}$ ) assertiert wird. Wird der Nebensatz dagegen als Teil der Nebenstruktur analysiert, gilt wie für die Nebensatztypen der Gruppe 2, dass sich 
B's Nein nur auf den Hauptsatzinhalt bezieht. In diesem Fall sollte auf der Skala ein hoher Wert gewählt werden, da q zutreffend ist. Ein Versuchsteilnehmer kann auch bemerken, dass die diskurssemantische Funktion der Nebensätze aus Gruppe 1 vom jeweils rekonstruierten Kontext abhängt. In einem solchen Fall ist zu erwarten, dass der Antwortwert 4 gewählt wird. Dies ist in der folgenden Hypothese noch einmal zusammengefasst:

\section{Hypothese H1:}

Die Nebensatztypen der Gruppe 2 drücken [-at-issue]-Inhalt aus und bleiben aus diesem Grund von der direkten Negation Nein unbetroffen. Die Nebensatztypen der Gruppe 1 können je nach Kontext [+/-at-issue]-Inhalt ausdrücken. Abhängig von der Art des durch den Probanden rekonstruierten Interpretationskontextes kann sich die direkte Negation Nein auf den Nebensatz beziehen oder nicht. Folglich ist zu erwarten, dass die Nebensätze aus Gruppe 1 im Schnitt einen niedrigeren Mittelwert (bei höherer Standardabweichung) erzielen als die Nebensätze aus Gruppe 2, kurz:

\section{Mittelwert Gruppe $1<$ Mittelwert Gruppe 2}

In Abschnitt 3.2.2.3 wurde dem Testdesign von Xue/Onea (2011) vorgeworfen, dass die [-at-issue]-Lesart bei den Testsätzen, mit denen das Verhalten von semifaktiven Komplementsätzen in Bezug auf einen direkten Widerspruch überprüft wurde, nicht sinnvoll ist (vgl. Beispiel (132), hier wiederholt als (140)):

(140) Petra weiß, dass Hans die Kekse gestohlen hat.

a. [-at-issue]-Lesart: Ja, und Hans hat sie nicht gestohlen.

b. [+at-issue]-Lesart: Nein, Hans hat sie gar nicht gestohlen.

Die [+at-issue]-Lesart lässt im Testdesign von Xue/Onea (2011) offen, ob die Hauptsatzproposition zutrifft oder nicht, wohingegen die [-at-issue]-Lesart bei gleichzeitiger Negation der Nebensatzproposition assertiert, dass die Hauptsatzproposition zutrifft. Da unklar ist, wie Petra etwas wissen kann, das nicht zutrifft, ist in diesem Fall lediglich die [+at-issue]-Lesart sinnvoll, was die hohe Rate an [+at-issue]Antworten für semifaktive Komplementsätze bei Xue/Onea (2011) erklären könnte. Diese Schwäche im Testdesign wird in der vorliegenden Studie umgangen, indem sowohl bei der [+at-issue]- als auch bei der [-at-issue]-Lesart offen bleibt, ob die Hauptsatzproposition zutrifft, was bei Xue/Onea (2011) nur bei der [+at-issue]Lesart der Fall war und demnach zu einer Antwortpräferenz geführt haben könnte. Im Rahmen der vorliegenden Studie wurden die acht unterschiedlichen Nebensätze aus Gruppe 1 und Gruppe 2 getestet. Es liegt demnach ein Testdesign mit acht Konditionen vor, die sich wie folgt zusammensetzen:

(141) Con 1: Komplementsatz, Brückenverb

Con 2: Semifaktiver Komplementsatz

Con 3: Faktiver Komplementsatz

Con 4: Temporalsatz 
Con 5: Weil-Satz

Con 6: Appositiver Relativsatz

Con 7: Restriktiver Relativsatz, definites Antezedens

Con 8: Restriktiver Relativsatz, indefinites Antezedens

Jeweils vier Konditionen können zu einer Gruppe zusammengefasst werden, und zwar bilden die Konditionen 3, 4, 6 und 7 die Gruppe der [-at-issue]-Inferenzen, wohingegen die Konditionen 1, 2, 5 und 8 in die Gruppe der [+/-at-issue]-Inferenzen gehören.

Dem Testdesign liegt demnach mit den unterschiedlichen Nebensätzen eine unabhängige Variable mit 8 Konditionen zugrunde, während die Bewertung der Versuchsteilnehmer auf der Skala von 1-7 die abhängige Variable bildet. Pro Kondition wurden sechs Stimuli getestet, bei acht Nebensatztypen ergibt sich daraus ein experimentelles Material bestehend aus 48 Items. Alle Testsätze wurden genau auf Störvariablen hin untersucht. Zusätzlich zu den Testsätzen enthielt der Fragebogen noch 12 Filler, welche gleichzeitig als Kontrollsätze der Überprüfung, ob die Versuchspersonen die Aufgabe und die Bewertungsskala richtig verstanden haben, dienten. Vier der Kontrollsätze waren eindeutig mit 1, vier mit 7 und weitere vier Kontrollsätze eindeutig mit $4 \mathrm{zu}$ bewerten (siehe Anhang). Auf ein zusätzliches Fillerset zur Maskierung der Forschungsfrage wurde verzichtet, da die Gefahr, dass die Versuchspersonen das Testdesign durchschauen und eine eigene Hypothese generieren, bei acht untereinander sehr heterogenen Konditionen gering ist. Zudem wären mehr als 12 Filler nur zulasten der Teststärke möglich gewesen, da der Fragebogen sonst zu lang geworden wäre.

In einem Within-Subject-Design wurden sämtliche Items allen Versuchspersonen in randomisierter Reihenfolge vorgelegt. Um Positionseffekten vorzubeugen, wurden zwei unterschiedliche Versionen des Fragebogens erstellt. Als Versuchspersonen nahmen 84 Studierende der Georg-August-Universität Göttingen teil, deren Muttersprache Deutsch ist. Die Erhebung fand im Rahmen von Seminaren der Deutschen Philologie statt und das Ausfüllen des Fragebogens veranschlagte etwa 20 Minuten, wobei den Versuchsteilnehmern keine zeitliche Begrenzung gesetzt wurde. 12 Fragebögen mussten von der Auswertung ausgenommen werden, da sie entweder nicht vollständig ausgefüllt wurden oder aber die Bewertung der Kontrollsätze den Schluss nahelegte, dass die Bewertungsskala nicht korrekt angewandt bzw. verstanden wurde. Ausgewertet wurden 72 Fragebögen (53f, 19m; $\mathrm{M}=22,42 ; \mathrm{SD}=3,83$ ).

\subsubsection{Ergebnisse}

Wie erwartet erzielten die Nebensätze aus Gruppe 1 mit durchschnittlich 3,88 (SD= 1,97) einen deutlich niedrigeren Mittelwert als die Nebensätze aus Gruppe 2, welche im Schnitt mit 6,43 (SD=1,08) bewertet wurden (vgl. Abbildung 3.1, die Fehlerbalken entsprechen der Standardabweichung). Zur Überprüfung der statistischen Bedeutsamkeit dieser Unterschiede wurde ein Lineares Gemischtes Modell mit maximaler random-effect-Struktur berechnet (d.h. mit random intercepts und random 
slopes für Items und Probanden, vgl. dazu Barr et al. 2013). Für den Vergleich von Gruppe 1 vs. Gruppe 2 ergab sich ein Wert von $|t|=14,15$, das Ergebnis weist den Unterschied folglich als signifikant aus. ${ }^{28}$ Dies steht im Einklang mit den Ergebnissen aus Abschnitt 2.2, denen zufolge die Nebensatztypen aus Gruppe 2 keinen [+at-issue]-Inhalt ausdrücken können und demnach von einem direkten Widerspruch unbetroffen bleiben. Damit bestätigen die Ergebnisse der Fragebogenstudie Hypothese H1.

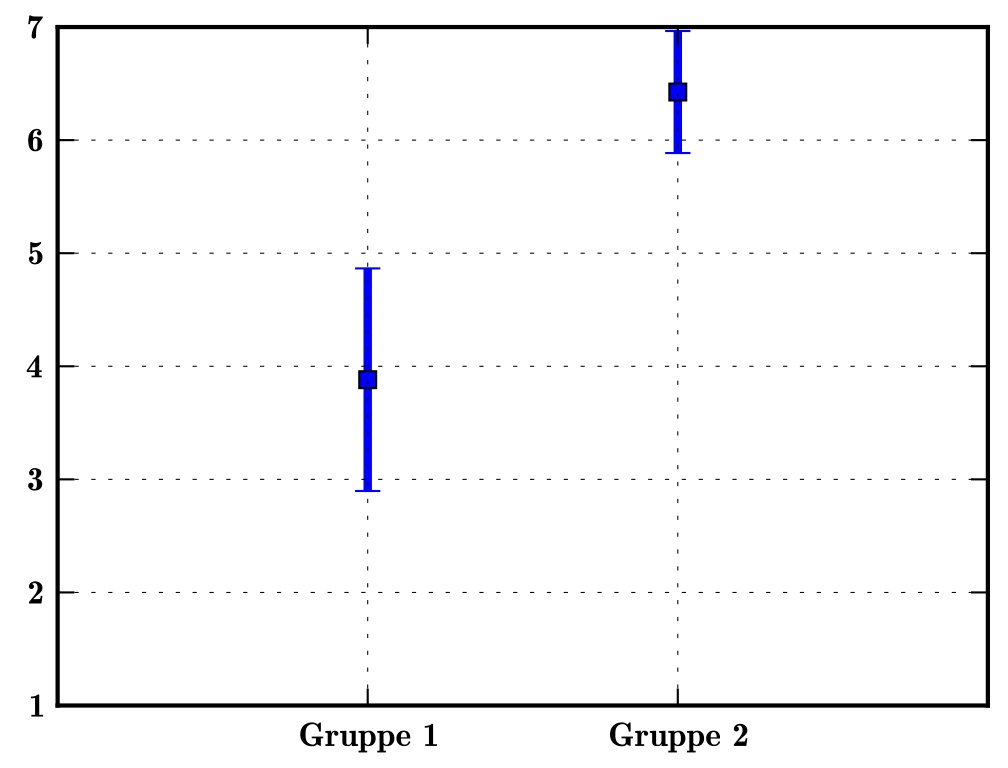

Abbildung 3.1.: Ergebnisse des Fragebogens nach Gruppen

Neben der Betrachtung der Mittelwerte lohnt sich ein Blick auf die Häufigkeitsverteilung der Bewertungen. In Abbildung 3.2 wird ersichtlich, dass die Nebensatztypen der Gruppe 2 in fast 90 Prozent aller Fälle mit 6 oder 7 bewertet wurden und somit mit einer sehr hohen Wahrscheinlichkeit von der direkten Negation unbetroffen blieben. Dagegen zeichnen sich die Bewertungen der Nebensätze aus Gruppe 1 durch eine deutlich höhere Variabilität auf, wobei die am häufigsten gewählten Werte 4, 7 und 1 sind. Dies unterstützt die weiter oben formulierte Annahme, dass die Nebensätze aus Gruppe 1 ambig sind, da sie eine [+at-issue]- und eine [-at-issue]-Lesart zulassen, und die gewählte Antwort somit vom jeweils rekonstruierten Diskurskontext abhängt. Dazu passt, dass die Nebebsätze aus Gruppe 1 eine deutlich höhere Standardabweichung aufweisen als die Sätze aus Gruppe 2 (1,97 im Vergleich zu $1,08)$.

${ }^{28}$ Ich folge Kliegl et al. (2010) in der Annahme, dass das Signifikanzkriterium beim Linearen Gemischten Modellen bei $|t|>2$ liegt. Alle absoluten t-Werte $>2$ beschreiben also signifikante Effekte. 


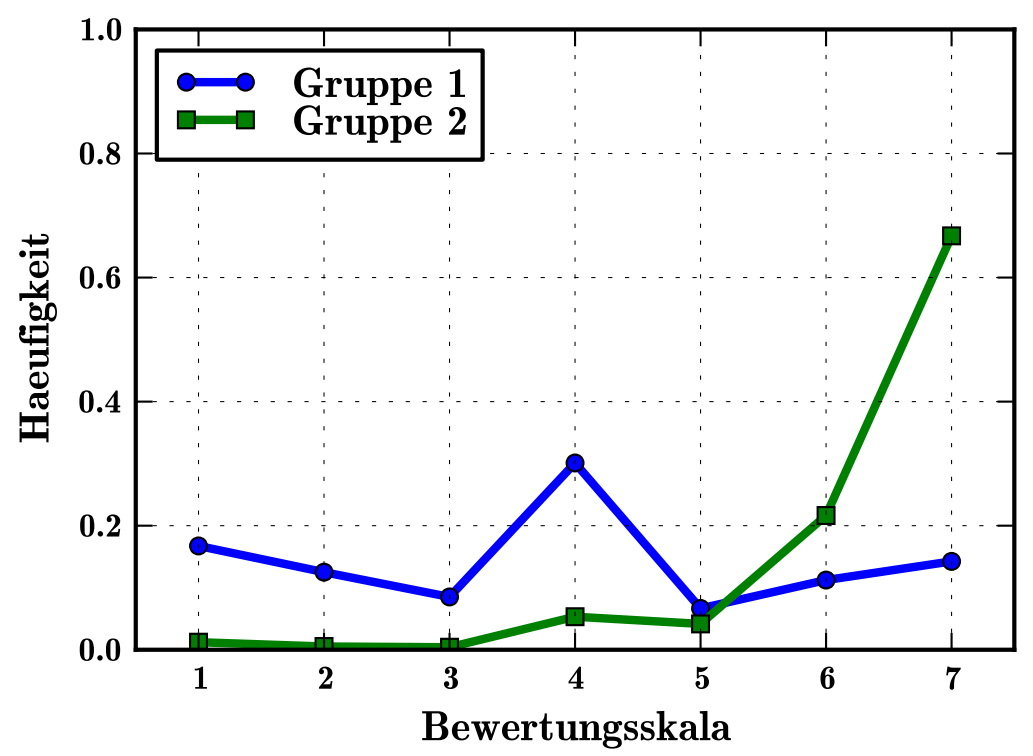

Abbildung 3.2.: Häufigkeitsverteilung nach Gruppen

Betrachten wir nun die aufgeschlüsselten Ergebnisse für die einzelnen Konditionen. In Abbildung 3.3 sind die Mittelwerte für die einzelnen Konditionen dargestellt.

Die Auswertung zeigt, dass innerhalb der Gruppe 2 nur ganz geringfügige Unterschiede bestehen. Die entsprechenden vier Konditionen erzielten ähnliche Werte und wurden von einer Mehrheit der Probanden als [-at-issue] interpretiert, wie aus Tabelle 3.4 noch einmal ersichtlich wird. Die Standardabweichung ist für alle vier Konditionen in etwa gleich hoch. Somit können wir festhalten, dass Hypothese H1 durch die Ergebnisse der Fragebogenstudie nicht nur für die Gruppe 2 insgesamt, sondern auch für die vier einzelnen Konditionen, aus denen sich Gruppe 2 zusammensetzt, bestätigt wird.

\begin{tabular}{|l||c|c|c|}
\hline Kondition & Mittelwert & Standardabweichung & N \\
\hline \hline 3 (Faktiv.) & 6,44 & 1,13 & 432 \\
\hline 4 (Temporals.) & 6,31 & 1,01 & 432 \\
\hline 6 (App. Rel.) & 6,56 & 0,93 & 432 \\
\hline 7 (Rest. Rel. def.) & 6,39 & 1,12 & 432 \\
\hline insgesamt & 6,43 & 1,08 & 1728 \\
\hline
\end{tabular}

Tabelle 3.4.: Ergebnisse des Fragebogens nach Konditionen, Gruppe 2

Etwas größere Unterschiede bestehen dagegen zwischen den Ergebnissen der einzelnen Konditionen innerhalb von Gruppe 1 (vgl. Tabelle 3.5). Während Komplementsätze von Brückenverben mit 3,56 den niedrigsten Wert (bei gleichzeitig geringster Standardabweichung) erzielten, wurden die semifaktiven Komplementsätze im Schnitt mit 4,26 (SD=2,08) bewertet. Der Unterschied zwischen dem Mittelwert von semifaktiven Komplementsätzen und den anderen Nebensatztypen aus Gruppe 


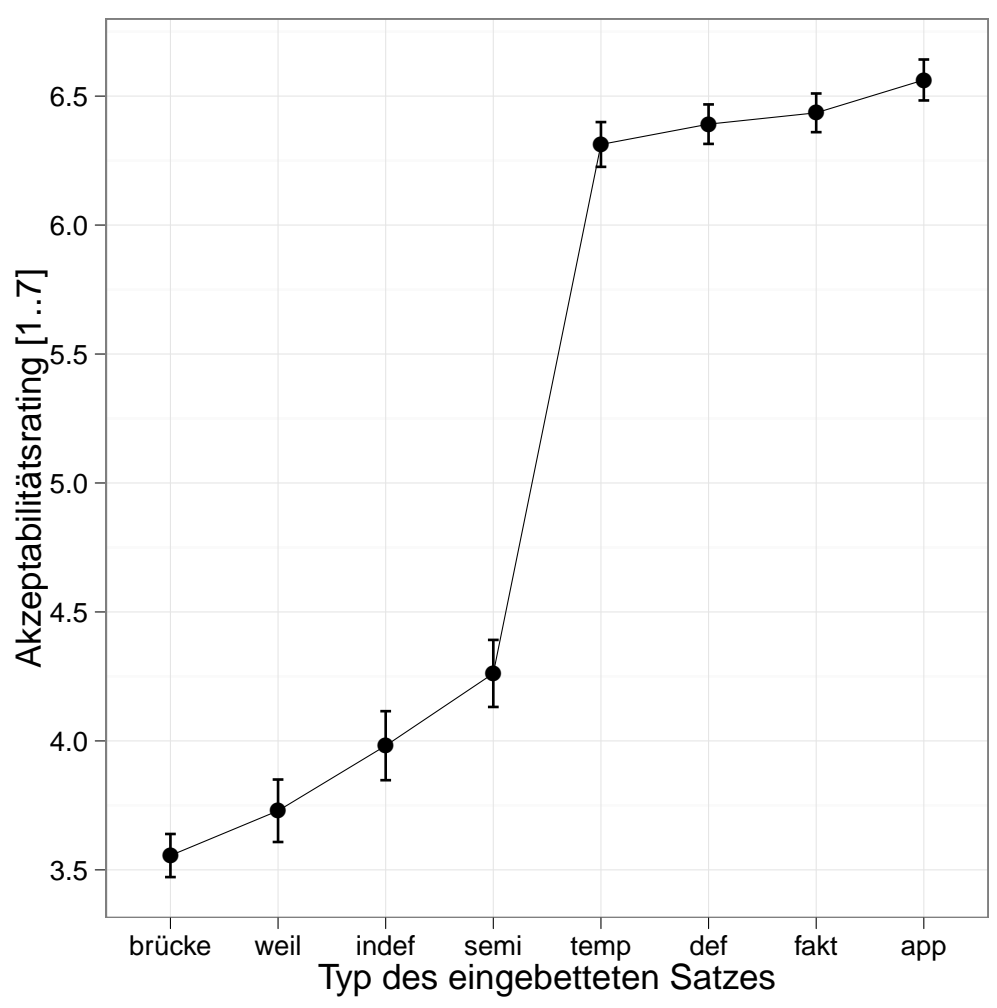

Abbildung 3.3.: Ergebnisse des Fragebogens nach Konditionen

1 erweist sich als signifikant, wie mit einem Linearen Gemischtes Modell mit maximaler random-effect-Struktur berechnet werden konnte $(|t|=7,92)$. Dass der Unterschied zwischen den semifaktiven Komplementsätzen und den Sätzen aus Gruppe 2 (durchschnittlicher Mittelwert $=6,43, \mathrm{SD}=1,08)$ aber ebenfalls signifikant ist $(|\mathrm{t}|=$ 12,75), zeigt, dass keine Neueinordnung der semifaktiven Nebensätze in Gruppe 2 vorzunehmen ist.

\begin{tabular}{|l|c|c|c|}
\hline Kondition & Mittelwert & Standardabweichung & $\mathbf{N}$ \\
\hline \hline 1 (Brückenverb) & 3,56 & 1,45 & 432 \\
\hline 2 (Semifaktiv) & 4,26 & 2,08 & 432 \\
\hline 5 (Weil-Satz) & 3,73 & 2,10 & 432 \\
\hline 8 (Rest. Rel. indef.) & 3,98 & 2,10 & 431 \\
\hline insgesamt & 3,88 & 1,97 & 1727 \\
\hline
\end{tabular}

Tabelle 3.5.: Ergebnisse des Fragebogens nach Konditionen, Gruppe 1

\subsubsection{Diskussion}

Die Ergebnisse der Fragebogenstudie zeigen eindeutig, dass dem Inhalt der vier Nebensatztypen aus Gruppe 2 nicht direkt widersprochen werden kann. Aufbauend 
auf den Ergebnissen von Roberts et al. (2009) und Simons et al. (2011) wird dies dahingehend interpretiert, dass die entsprechenden vier Nebensatztypen nie Teil der Hauptstruktur sein können. Zudem konnte ein statistisch signifikanter Unterschied zwischen der Wahrscheinlichkeit, dass ein direkter Widerspruch auf den Inhalt eines Nebensatzes aus Gruppe 2 und der Wahrscheinlichkeit, dass der Widerspruch auf einen Nebensatz aus Gruppe 1 bezogen wird, festgestellt werden. Es hat sich gezeigt, dass sich die Nebensätze aus Gruppe 2 alle sehr ähnlich verhalten, da sie alle mit einer in etwa gleich hohen Wahrscheinlichkeit von der direkten Negation unbetroffen bleiben und demnach von den Probanden als [-at-issue] interpretiert wurden. Auch die Häufigkeitsverteilung der Bewertungen ist für die einzelnen vier Nebensatztypen aus Gruppe 2 sehr ähnlich, wie Abbildung 3.4 zeigt. Alle vier Konditionen wurden in etwa 90 Prozent der Fälle mit 6 oder 7 bewertet, d.h. als [-at-issue], interpretiert. Dies stützt die in den Abschnitten 2.1.3 und 2.2.5 formulierte Ansicht, derzufolge die vier Nebensatztypen aus Gruppe 2 alle eine konventionelle Markierung für ihre Zugehörigkeit zur Nebenstruktur aufweisen.

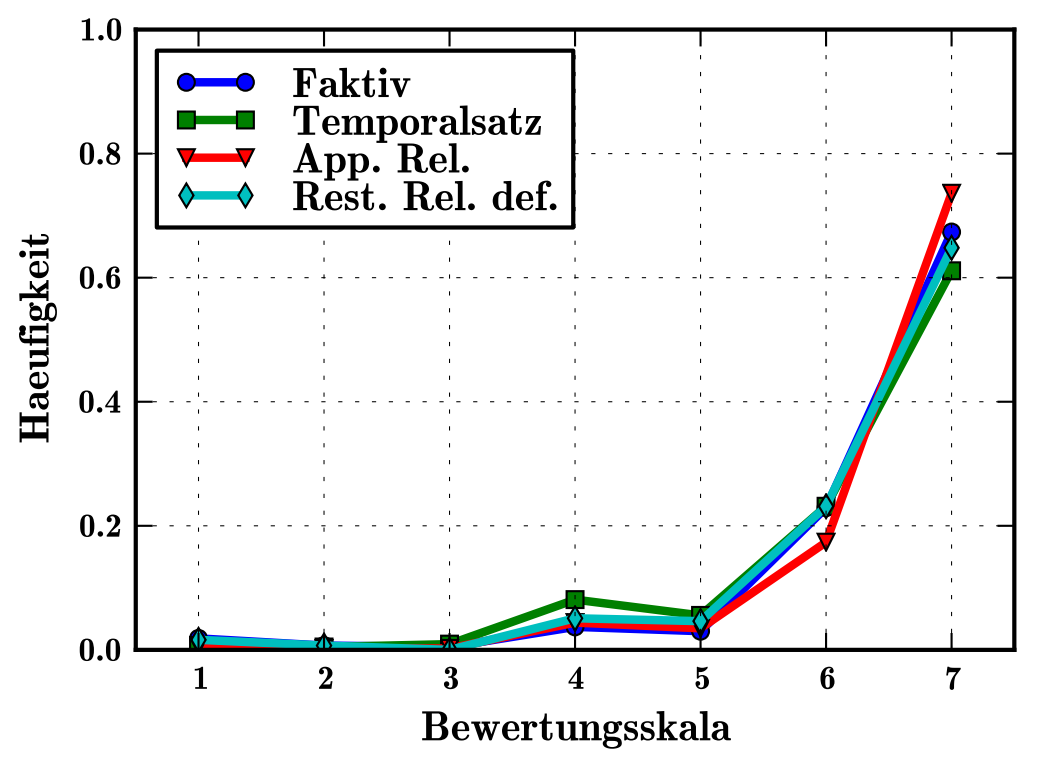

Abbildung 3.4.: Häufigkeitsverteilung Gruppe 2

Etwas größer sind dagegen die Unterschiede in der Bewertung der vier Nebensatztypen aus Gruppe 1, und zwar sowohl bezogen auf die Mittelwerte (vgl. Tabelle 3.5) als auch die Häufigkeitsverteilung, wie Abbildung 3.5 zeigt.

Die Komplemente von Brückenverben wurden präferiert als ambig interpretiert und die entsprechenden Sätze in den meisten Fällen mit 4 bewertet. Die Probanden wussten demnach nicht, ob der Widerspruch Nein auch auf den Nebensatzinhalt bezogen werden muss oder nicht. Dies ist nicht weiter verwunderlich, da Brückenverben wie glauben oder finden eine indirekte Assertion einbetten, deren Inhalt der jeweilige Sprecher nicht unbedingt vertreten muss (vgl. dazu auch Julien 2007, 14), zudem war das Matrixsubjekt in keinem der sechs Testsätze identisch mit dem Sprecher. 


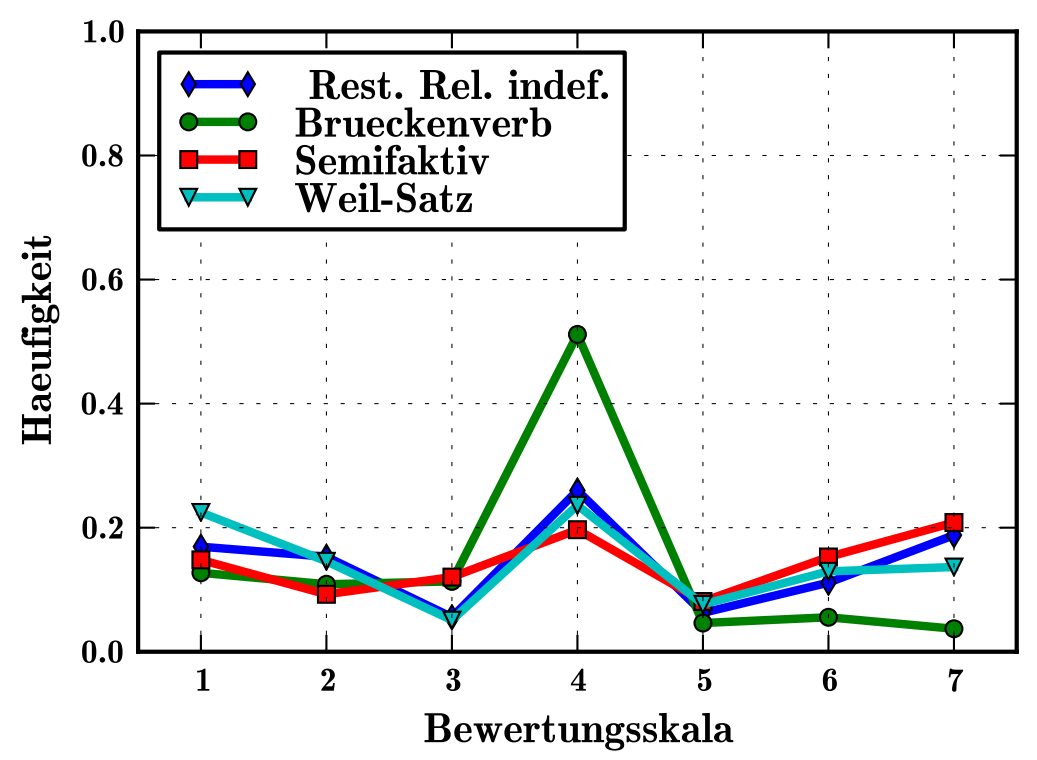

Abbildung 3.5.: Häufigkeitsverteilung Gruppe 1

Anders als bei den Komplementen von Brückenverben, welche in den meisten Fällen mit 4 und nur mit einer geringen Wahrscheinlichkeit mit 6 oder 7 bewertet wurden, sieht es bezogen auf die semifaktiven Komplementsätze aus. Wie in Abbildung $3.5 \mathrm{zu}$ sehen ist, erzielten diese am häufigsten den Wert 7 und wurden von allen vier Nebensatztypen aus Gruppe 1 mit der größten Wahrscheinlichkeit als [-at-issue] interpretiert. Der Unterschied zwischen dem von semifaktiven Komplementsätzen erzielten Mittelwert und dem der restlichen drei Konditionen aus Gruppe 1 erwies sich zudem als signifikant (vgl. Abschnitt 3.2.3.2). Dass die semifaktiven Testsätze mit einer höheren Wahrscheinlichkeit als [-at-issue] interpretiert wurden als die anderen Nebensatztypen aus Gruppe 1, kann darauf zurückgeführt werden, dass derartige Sätze typischerweise präsupponiert sind und nur in bestimmten Kontexten ihre Faktivität verlieren (vgl. Beispiel (43) sowie Hooper/Thompson 1973 und Karttunen 1971). Diese nicht-faktive Lesart jedoch gilt es zu rekonstruieren, damit der Nebensatz Teil der Hauptstruktur ist und somit von der direkten Negation betroffen sein kann. ${ }^{29}$ Dennoch ist keine Neueinordung der semifaktiven Komplementsätze in Gruppe 2 vorzunehmen - während die Nebensatztypen der Gruppe 2 nämlich grundsätzlich nicht Teil der Hauptstruktur sein können, besteht diese Möglichkeit für semifaktive Komplementsätze zumindest (der Unterschied zwischen den semifaktiven Komplementsätzen und dem Mittelwert von Gruppe 2 ist signifikant, vgl. Abschnitt 3.2.3.2), zudem weisen sie in den Grundzügen die gleiche Häufigkeitsverteilung auf wie die

\footnotetext{
${ }^{29}$ Bentzen (2009) geht auf Grundlage von (rein introspektiv erhobenen) norwegischen Daten davon aus, dass dem Inhalt von semifaktiven Komplementsätzen grundsätzlich nicht direkt widersprochen werden kann. Diese Ansicht konnte mit der vorliegenden Fragebogenstudie für das Deutsche nicht bestätigt werden, fest steht jedoch, dass die Lesart, in der ein direkter Widerspruch auf einen semifaktiven Komplementsatz bezogen wird, schwerer rekonstruierbar ist.
} 


\section{Typische Eigenschaften der Nebenstruktur}

anderen drei Nebensatztypen aus Gruppe 1.

Anders als bei Gruppe 2 ist die Wahrscheinlichkeit, dass ein abhängiger Satz als Teil der Hauptstruktur analysiert wird, für die vier relevanten Nebensatztypen unterschiedlich hoch. Dies deutet darauf hin, dass wir es hier mit einem graduellen Phänomen zu tun haben, was bedeutet, dass die Nebensatztypen aus Gruppe 1 unterschiedliche präferierte Lesarten besitzen. Die Ergebnisse der Fragebogenstudie zeigen aber auch, dass sich alle Nebensätze aus Gruppe 1 ein entscheidendes Merkmal teilen, indem sie prinzipiell [+at-issue] sein können. Dagegen verhalten sich die Nebensatztypen aus Gruppe 2 weitgehend homogen, indem sie mit einer sehr hohen Wahrscheinlichkeit als [-at-issue] interpretiert werden. Dieses Ergebnis deckt sich auf den ersten Blick nicht mit den Beobachtungen Xue/Onea (2011), die zu dem Schluss kommen, dass [-at-issue]-Inhalte unterschiedlich stark dazu neigen, unter Einbettung zu projizieren. Diese Diskrepanz kann aber folgendermaßen erklärt werden: Unter den von Xue/Onea (2011) vier untersuchten Triggern befinden sich mit wissen und erfahren zwei semifaktive Verben (vgl. Tabellen 3.2). Wir haben aber bereits in Abschnitt 2.2.2 gesehen, dass semifaktive Verben sehr wohl in der Lage sind, einen Nebensatz einzubetten, der [+at-issue]-Inhalt ausdrückt, weswegen sie hier zu Gruppe 1 gezählt werden. Somit ist es nicht verwunderlich, dass die entsprechenden Testsätze in dem Experiment von Xue/Onea (2011) nicht in jedem Kontext projizieren. Da Xue/Onea (2011) semifaktive Komplementsätze, die je nach Kontext auch zur Hauptstruktur gehören können, mit den Präsuppositionsauslösern auch und wieder vergleichen, kommen sie zu dem Ergebnis, dass die Projektionswahrscheinlichkeit unterschiedlich hoch ist. Vergleicht man nun jedoch nur solche Inferenzen miteinander, die wie die Nebensätze aus Gruppe 2 und anders als semifaktive Nebensätze grundsätzlich keinen [+at-issue]-Inhalt ausdrücken können, lässt sich keine graduelle Abstufung feststellen. Vielmehr verhalten sich die Inferenzen alle gleich, indem sie immer als Teil der Nebenstruktur interpretiert werden.

Die Ergebnisse der Fragebogenstudie belegen abermals, dass abhängige Sätze anders als von u.a. Bartsch (1978), Hartmann (1984), Posner (1972) und von Stutterheim (1989, 168) postuliert, durchaus zur Hauptstruktur gehören können, wie bereits Brandt (1989, 1996), Holler (2009) und Simons (2007) beobachtet haben. Mittels der Beobachtung von Roberts et al. (2009) und Simons et al. (2011), derzufolge nur [+at-issue]-Inhalten direkt widersprochen werden kann, konnte gezeigt werden, dass die vier Nebensatztypen der Gruppe 1 mit einer unterschiedlich hohen Wahrscheinlichkeit zur Hauptstruktur gezählt werden. Es wäre sicherlich interessant, in einer weiteren empirischen Studie bezogen auf die Nebensätze der Gruppe 1 den Zusammenhang zwischen At-issueness und der Sensibilität für direkte Widerspruchsstrategien zu überprüfen. Für diesen Zweck könnte beispielsweise ein Kontext vorgegeben werden, aus welchem eindeutig ersichtlich ist, ob der Nebensatz zur Haupt- oder Nebenstruktur gehört. 


\subsection{Zusammenfassung des Kapitels}

Aufgrund der Beobachtung, dass nur einige Nebensätze dazu verwendet werden können, die in einem Diskurs jeweils aktuelle $\mathrm{Q}$ zu beantworten, wurden in Abschnitt 2.2.5 zwei Arten von Nebensatztypen voneinander unterschieden. Die Nebensätze der Gruppe 1 können je nach Kontext [+at-issue]- oder [-at-issue]-Inhalt ausdrücken. Nur wenn sie Teil der Nebenstruktur sind, projizieren sie unter Einbettung, andernfalls überlebt die eingebettete Proposition nicht. Dies deckt sich mit der in Roberts et al. (2009) und Simons et al. (2011) vertretenen Annahme, dass nur [-at-issue]-Inhalte projizieren. Des Weiteren konnte mithilfe einer Fragebogenstudie gezeigt werden, dass die Nebensätze der Gruppe 1 prinzipiell sensibel sind für einen direkten Widerspruch. Auch dies ergibt sich direkt aus Roberts et al. (2009), die vorhersagen, dass nur [+at-issue]-Inhalten direkt widersprochen werden kann. Die Tatsache, dass die vier Nebensatztypen aus Gruppe $1 \mathrm{zu}$ einem unterschiedlichen Grad sensibel für direkte Widerspruchsstrategien sind, wurde als Zeichen dafür gedeutet, dass die Sätze mit einer unterschiedlich hohen Wahrscheinlichkeit [+atissue]-Inhalt ausdrücken.

Die Nebensätze der Gruppe 2 können dagegen nicht dazu verwendet werden, die aktuelle Q zu beantworten (vgl. Abschnitt 2.2). Daraus ergibt sich die Vorhersage, dass ihr Inhalt von inferenztilgenden Operatoren ignoriert wird und demnach projizieren sollte. Dass dies zutreffend ist, wurde in Abschnitt 3.1.2 gezeigt. Eine weitere Vorhersage wurde mithilfe einer Fragebogenstudie untersucht. Diese ergab, dass dem Inhalt von Nebensätzen, welche immer [-at-issue] sind, nicht direkt widersprochen werden kann. Dies wurde als Bestätigung der in Abschnitt 2.2.5 formulierten Annahme, derzufolge die Nebensätze der Gruppe 2 konventionelle Markierungen für ihre Zugehörigkeit zur Nebenstruktur tragen, gewertet. Mit dem diskursstrukturellen Unterschied zwischen den beiden Nebensatztyp gehen demnach weitere Unterschiede einher, welche in Tabelle 3.6 noch einmal zusammengefasst sind.

\begin{tabular}{|l||c|c|c|}
\hline Nebensatztyp & [at-issue] & Projektion & dir. Widerspruch \\
\hline \hline Komplementsatz, Brückenverb & $+/-$ & $+/-$ & $+/-$ \\
\hline Semifaktiver Komplementsatz & $+/-$ & $+/-$ & $+/-$ \\
\hline Faktiver Komplementsatz & - & + & - \\
\hline Temporaler Adverbialsatz & - & + & $+/-$ \\
\hline Adverbialsatz mit weil & $+/-$ & $+/-$ & - \\
\hline Appositiver Relativsatz & - & + & $+/-$ \\
\hline $\begin{array}{l}\text { Restriktiver Relativsatz, defi- } \\
\text { nites Antezedens }\end{array}$ & - & + & - \\
\hline $\begin{array}{l}\text { Restriktiver Relativsatz, inde- } \\
\text { finites Antezedens }\end{array}$ & $+/-$ & $+/-$ & \\
\hline
\end{tabular}

Tabelle 3.6.: Typische Eigenschaften der Haupt- und Nebenstruktur 
3. Typische Eigenschaften der Nebenstruktur 


\section{Präsupponiertheit und At-issueness}

In Kapitel 3 wurde gezeigt, dass Eigenschaften, die traditionell zur Ermittlung von Präsuppositionen verwendet werden, nicht nur auf Präsuppositionen, sondern auf alle [-at-issue]-Inferenzen zutreffen. So hat sich herausgestellt, dass auch appositive Relativsätze oder expressive Ausdrücke projizieren, ohne dabei präsupponiert zu sein, zudem kann ihnen nicht direkt widersprochen werden. Daraus ergeben sich nun die Fragen, in welchem Verhältnis Präsupponiertheit zu dem Konzept der At-issueness steht und auf welche Weise präsupponierte Inhalte ermittelt werden können. Um diese Fragen beantworten zu können, wird zunächst in Kapitel 4.1 untersucht, welche distinktiven Eigenschaften Präsuppositionen aufweisen und welche Konsequenzen sich daraus für die Abgrenzung von Präsuppositionen zu [+at-issue]und [-at-issue]-Inhalten ergeben. In Kapitel 4.2 wird dann anhand dieser Eigenschaften überprüft, welche der Nebensätze der Gruppen 1 und 2 präsupponiert sind.

\subsection{Distinktive Merkmale von Präsuppositionen}

Im Folgenden werden drei Eigenschaften diskutiert, die Präsuppositionen von der Forschungsliteratur zugeschrieben werden, nämlich dass sie wahr sein müssen, eine im Common Ground gegebene Proposition ausdrücken und anaphorisch sind. Die letzte Eigenschaft wird dabei etwas ausführlicher dargestellt, da die anaphorische Präsuppositionstheorie in Abschnitt 9.1.3 verwendet wird. Im vorliegenden Kapitel wird untersucht, ob es sich bei den drei Eigenschaften um distinktive Eigenschaften handelt, die zur Abgrenzung zwischen Präsuppositionen und dem Konzept der Atissueness geeignet sind, und welches Verhältnis zwischen Präsupponiertheit und Atissueness daraus folgt.

\subsubsection{Präsuppositionen müssen wahr sein}

Klassischerweise werden Präsuppositionen vor allem über eine Bedingung an den Wahrheitswert definiert. Nach u.a. Van Fraasen (1971) und Strawson (1950) unterscheiden sich Präsuppositionen von anderen Inhalten darin, dass sie wahr sein müssen, damit der Gesamtsatz eine wahrheitskonditionale Bedeutung hat. Betrachten wir dazu ein Beispiel. Bei Satz (142-b) handelt es sich um eine Präsupposition von Beispiel (142-a), die durch das faktive Verb ignorieren ausgelöst wird. 
a. Peter ignoriert, dass Maria in Mainz ist.

b. Maria ist in Mainz.

Nach Potts (2011) denotiert ein Satz wie (142-a), der zwei Inferenzen auf zwei unterschiedlichen Bedeutungsebenen transportiert, ein Bedeutungstupel bestehend aus den folgenden vier Möglichkeiten. Der erste Wert entspricht dabei der $[+$ atissue]-Bedeutung (= Peter ignoriert Marias Anwesenheit in Mainz), wohingegen der Wahrheitswert der eingebetteten Inferenz in der Klammer jeweils an zweiter Stelle steht. Es können also beide Inferenzen wahr oder beide falsch sein oder aber die beiden Inferenzen besitzen unterschiedliche Wahrheitswerte.

$$
<1,1>,<0,1>,<1,0>,<0,0>
$$

Handelt es sich bei der eingebetteten Inferenz nun um eine Präsupposition, ist die Gesamtäußerung nur dann wahrheitskonditional interpretierbar, wenn die Präsupposition wahr ist. In den beiden Fälle $<1,0>$ und $<0,0>$ kann nicht darüber entschieden werden, ob Satz (142-a) wahr oder falsch ist. ${ }^{30}$ Im Unterschied dazu muss ein durch ein Brückenverb eingebetteter Satz nicht wahr sein, damit der Gesamtsatz wahrheitskonditional interpretiert werden kann. So kann die im folgenden Beispiel eingebettete Proposition auch ohne Weiteres den Wahrheitswert 0 besitzen, dennoch ließe sich der Gesamtsatz wahrheitskonditional beurteilen.

$$
\text { Peter hat erzählt, dass Maria in Mainz ist. }
$$

Ähnlich wie faktive Verben lösen auch definite NPn eine Präsupposition aus. Die klassische Beschreibung der Semantik von definiten NPn lässt sich auf die Kennzeichnungstheorie nach Russell (1905) zurückführen, welche davon ausgeht, dass NPn referierende Ausdrücke sind. Eine definite NP muss dabei (i) eine Existenzbedingung und (ii) eine Einzigkeitsbedingung befolgen. Die Existenzbedingung besagt nichts anderes, als dass es ein Individuum im entsprechenden Modell gibt, auf das die Beschreibung zutrifft, wohingegen Bedingung (ii) ausdrückt, dass es sich um genau ein Individuum handelt. Bezogen auf den Beispielsatz (145-a) aus Russell (1905) bedeutet dies, dass es ein Individuum x gibt, für das gilt, dass es der König von Frankreich ist (hier abgekürzt als Prädikat K). Für alle y, auf die die gleiche Beschreibung $\mathrm{K}$ zutrifft, gilt $\mathrm{y}=\mathrm{x}$, da es nicht mehr als ein Individuum mit der Eigenschaft K geben darf. Außerdem wird x die Eigenschaft B, also Kahlköpfigkeit, zugeschrieben. Die logische Form von Satz (145-a) ist in (145-b) angegeben.

$$
\begin{aligned}
& \text { a. The king of France is bald. } \\
& \text { b. } \exists \mathrm{x}[\mathrm{K}(\mathrm{x}) \wedge \forall \mathrm{y}[\mathrm{K}(\mathrm{y}) \rightarrow \mathrm{x}=\mathrm{y}] \wedge \forall \mathrm{x}[\mathrm{K}(\mathrm{x}) \rightarrow \mathrm{B}(\mathrm{x})]]
\end{aligned}
$$

Aus der Existenzbedingung $\exists x K x$ leitet Strawson (1950) die durch definite Ausdrücke ausgelöste Präsupposition ab. Laut Strawson (1950) präsupponiert eine defi-

${ }^{30}$ Aus diesem Grund wird häufig neben den zwei Wahrheitswerten wahr und falsch die Notwendigkeit eines dritten Wertes, nämlich undefiniert (dargestellt als ,\#'), postuliert (vgl. u.a. die three-valued logic von Van Fraasen 1971). 
nite NP das Vorhandensein von genau einem Individuum im jeweiligen Modell, auf das die entsprechende Eigenschaft zutrifft. Nur wenn diese Präsupposition erfüllt ist, ist eine wahrheitskonditionale Interpretation des Gesamtsatzes möglich. Ob Satz (145-a) wahr oder falsch ist, kann demnach nur dann beurteilt werden, wenn wir davon ausgehen, dass es einen (einzigen) König von Frankreich gibt.

Präsuppositionen müssen folglich wahr sein, damit der Gesamtsatz eine wahrheitskonditionale Bedeutung hat. Anders als bei den in Kapitel 3 diskutierten Eigenschaften, die das Projektionsverhalten und mögliche Widerspruchsstrategien betreffen, scheint es sich dabei tatsächlich um eine distinktive Eigenschaft von Präsuppositionen zu handeln, durch die sie sich von anderen [-at-issue]-Inhalten unterscheiden. Dies zeigt sich u.a. daran, dass appositive Relativsätze, welche immer Teil der Nebenstruktur sind, keine solche Auflage an den Wahrheitswert erfüllen müssen, damit der Gesamtsatz wohlgeformt ist. Beispielsweise kann der appositive Relativsatz in (146) auch falsch sein und dennoch ist es möglich, dem Hauptsatz einen Wahrheitswert zuzuordnen, was auch Potts $(2011,3)$ beobachtet. So denotiert auch der folgende Beispielsatz das Bedeutungstupel (143), wobei alle vier Fälle wahrheitskonditional interpretierbar sind.

(146) Jap, der gerade in Indonesien ist, fährt gerne ans Meer.

Dies ist zu erwarten, da sich Appositionen ähnlich wie Expressiva verhalten, welche bei der Erstellung der wahrheitskonditionalen Semantik keine Rolle spielen (vgl. dazu Kaplan 1999, Kratzer 1999 und Potts 2005). Wir können also festhalten, dass Präsuppositionen alle Eigenschaften aufweisen, die auch auf [-at-issue]-Inferenzen zutreffen, wie beispielsweise die Projektion unter Einbettung, zusätzlich aber auch noch wahr sein müssen. Dies führt zu der Annahme, dass es sich bei Präsuppositionen um eine Untermenge der [-at-issue]-Inhalte handelt.

\subsubsection{Präsuppositionen müssen im Common Ground gegeben sein}

Präsuppositionen müssen nicht nur wahr sein: Seit den Untersuchungen von Stalnaker (1974) wird ebenfalls davon ausgegangen, dass Präsuppositionen bereits gegebene Informationen ausdrücken. Jede Äußerung wird vor dem Hintergrund des Common Ground gemacht. Dieser bezeichnet die Menge an Propositionen, welche die Konversationsteilnehmer als gegeben akzeptieren und über deren Zutreffen keine Diskussion notwendig ist. Der Common Ground umfasst die Menge an Welten, für die gilt, dass die akzeptierten Propositionen in ihnen wahr sind, das sogenannte Kontextset (vgl. dazu u.a. Karttunen 1974 und Stalnaker 1974, 1978). Der Common Ground bestimmt nun, was gesagt wird: Eine assertive Äußerung zielt auf ein Common Ground-Update ab. Akzeptieren die übrigen Diskursteilnehmer eine assertierte Proposition von S, so wird diese dem Common Ground hinzugefügt. Bezogen auf das Kontextset bedeutet dies, dass alle Welten, die mit dieser neu hinzugefügten Annahme nicht kompatibel sind, entfernt werden. Mit der Äußerung einer Frage dagegen 


\section{Präsupponiertheit und At-issueness}

fordert S den Gefragten dazu auf, den Common Ground seinerseits um die gewünschte Information zu erweitern bzw. mitzuteilen, welche Welten aus dem Kontextset zu entfernen sind (zur Semantik von Fragen vgl. u.a. Altmann 1993, Bierwisch 1980, Brandt 1992, Groenendijk/Stokhof 1985, Hamblin 1958, Hamblin 1973, Higginbotham 1996, Karttunen 1977, Lohnstein 2000, Lohnstein 2013, Reis/Rosengren 1991, Reis 1992 und Truckenbrodt 2004). Das Konzept der pragmatischen Präsupposition fußt nun auf der Intuition, dass Präsuppositionen Informationen ausdrücken, die zum Äußerungszeitpunkt bereits Teil des Common Ground sind. Ein Satz wie der folgende präsupponiert beispielsweise, dass jemand den Kuchen aufgegessen hat. Die durch den Spaltsatz ausgelöste Präsupposition stellt Anforderungen an den Common Ground, in dem die präsupponierte Proposition bereits enthalten sein muss. Das Kontextset darf zudem nur aus solchen Welten bestehen, in denen die Proposition wahr ist.

(147) Es war nicht Georg, der den Kuchen aufgegessen hat.

$\gg$ Jemand hat den Kuchen aufgegessen.

Etwas präsupponieren bedeutet demzufolge, etwas als Teil des Common Ground zu behandeln. Dementsprechend definieren u.a. Karttunen (1974) und Stalnaker (1974) Präsuppositionen als Anforderungen an den Kontext (vgl. auch Beaver 2001, Heim 1983 und Schlenker 2008).

Handelt es sich nun bei der Anforderung an den Common Ground um ein geeignetes Kriterium zur Unterscheidung zwischen präsupponierten und nicht-präsupponierten Inferenzen? Ein pragmatischer Präsuppositionsbegriff würde vorhersagen, dass präsupponierte Bedeutungsaspekte immer eine im Common Ground bereits enthaltene Information ausdrücken. Dass dies nicht auf alle [-at-issue]-Inferenzen zutrifft, haben wir bereits in Abschnitt 3.1.1 gesehen. Hier wurde argumentiert, dass appositive Relativsätze nicht präsupponiert sind, obwohl sie immer [-at-issue]-Inhalt ausdrücken, da sie keine im Kontext vorerwähnte Information ausdrücken können, wie bereits Holler $(2005,54)$ und Roberts et al. (2009) gezeigt haben. Die entsprechenden Beispiele aus (96) werden im Folgenden noch einmal wiederholt:

a. \#Ein Mann küsste eine Frau und diese Frau, die er übrigens küsste, war verheiratet.

b. Jap ist gerade in Indonesien. \#Jap, der (übrigens) gerade in Indonesien ist, fährt gerne ans Meer.

Die Beispiele zeigen, dass anhand der Common Ground-Anforderung prinzipiell zwischen Präsuppositionen und anderen [-at-issue]-Inferenzen unterschieden werden kann. Appositive Relativsätze projizieren genau wie präsupponierte Sätze, zudem können sie nicht die aktuell relevante Q beantworten, im Unterschied zu Präsuppositionen darf ihr Inhalt aber nicht vorerwähnt sein.

Zur Ermittlung von Präsuppositionen ist die Beobachtung, dass diese immer bereits Bekanntes ausdrücken, jedoch nur bedingt geeignet. So besteht häufig eine Diskrepanz zwischen dem, was die Diskursteilnehmer als Teil des Common Ground be- 
trachten, und dem, was ein Sprecher präsupponiert. Anders formuliert sind Präsuppositionen zum Äußerungszeitpunkt nicht immer schon bekannt, sondern können durchaus auch neue Informationen enthalten, ein Punkt, dem in der Akkommodationsforschung Rechnung getragen wird. Nehmen wir beispielsweise an, dass der folgende Satz als Antwort auf die Frage, was man als größte persönliche Leistung im vergangenen Jahr betrachtet, geäußert wird. In einer solchen Situation muss die Präsupposition keineswegs bereits Teil des Common Ground sein.

Ich habe mit dem Rauchen aufgehört.

$\gg$ Ich habe geraucht.

Ein weiteres prominentes Beispiel für eine informative Präsupposition bilden Verwendungen definiter NPn ohne vorherige Einführung durch ein indefinites Antezedens. Die NP meine Tochter in (150) präsupponiert, dass S eine Tochter hat. Der Satz kann nun aber ohne Weiteres in einem Kontext geäußert werden, in dem die Präsupposition noch nicht Teil des Common Ground ist.

(150) Ich muss heute leider früher gehen, meine Tochter ist krank. $\gg \mathrm{S}$ hat eine Tochter.

Nach Schwarz $(1998,468)$ handelt es sich dabei keineswegs um marginale Ausnahmen, sondern vielmehr um die zahlenmäßig häufigste Art der Verwendung von definiten NPn. Über 60 Prozent aller definiten NPn sollen demnach in Texten ohne Einführung durch ein indefinites Antezedens verwendet werden. Für eine pragmatische Präsuppositionstheorie, deren Hauptaussage darin besteht, dass Präsuppositionen immer im Common Ground gegeben sind, stellen derartige Fälle eine Herausforderung dar. Verwendungen von definiten NPn ohne Antezedens werden gewöhnlich unter dem Begriff des Bridging analysiert. Eine wichtige Beobachtung hierbei ist, dass eine definite NP ohne indefinites Antezedens über eine Relation mit dem vorherigen Kontext verbunden werden muss (vgl. dazu u.a. Asher/Lascarides 1998a, Burkhardt 2005, Clark 1975, Geurts 2011 und Schwarz 2000). Beispielsweise kann die NP der Gitarrist in dem folgenden Beispiel unter Bezug auf das indirekte Antezedens Konzert interpretiert werden. ${ }^{31}$

Ich war gestern auf einem Konzert. Der Gitarrist hat sich ständig verspielt.

Auch faktive Komplementsätze, welche in der Literatur unkontrovers als präsupponiert angesehen werden, können eine neue Information einführen. Die Äußerung des folgenden Satzes wäre laut Roberts et al. (2009) sogar markiert, wenn die eingebettete Proposition bereits im Common Ground gegeben wäre.

\footnotetext{
${ }^{31}$ Burkhardt (2006) hat gezeigt, dass sich solche Verwendungen definiter NPn im EEG-Experiment ähnlich wie diskursneue Referenten verhalten, indem sie eine verstärkte P600 auslösen. Dies widerspricht der Ansicht, derzufolge Präsuppositionen immer gegeben sein müssen. Gleichzeitig konnte Burkhardt (2006) aber auch nachweisen, dass gebridgte NPn auch Eigenschaften mit bekannten Referenten teilen, indem sie eine abgeschwächte N400 aufzeigen.
} 
We regret to inform you that your project has not been selected for further review.

Auch sententiale Präsuppositionen können demnach ähnlich wie definite NPn verwendet werden, ohne im Common Ground gegeben zu sein. U.a. Irmer (2009) argumentiert dabei dafür, dass Bridging-Relationen nicht nur bei der Referenz auf Entitäten, sondern auch bei der Referenz auf Ereignisse eine Rolle spielen können. Asher/Lascarides (1998b) und Asher/Lascarides (2003) formulieren die Idee, dass alle informativen Präsuppositionen wie definite NPn über eine Relation mit einem indirekten Antezedens verknüpft werden müssen, um interpretierbar zu sein, im Rahmen der Diskursrepräsentationstheorie SDRT aus, was im folgenden Abschnitt noch genauer diskutiert wird.

Abgesehen von Untersuchungen zu definiten NPn liegen bislang keine systematischen Korpusstudien darüber vor, wie häufig Verwendungen von informativen Präsuppositionen sind. Dies liegt wohl daran, dass sich nur sehr schwer untersuchen lässt, ob der Inhalt von beispielsweise einem faktiven Komplementsatz gegeben ist oder nicht. So besteht der Common Ground ja nicht nur aus bereits gemachten Äußerungen, sondern auch aus situativem, Kontext- und Weltwissen. Beispielsweise kann die durch ein faktives Verb ausgelöste Präsupposition in A's Äußerung in (153) auch deiktisch verankert werden.

Kontext: A kommt in den Flur und sieht, dass B gerade seinen Mantel anzieht.

A: Ich bedaure, dass du schon gehst.

Gauker (1998) sieht in der Existenz informativer Präsuppositionen einen Grund, Common Ground-Theorien zur Beschreibung von Präsuppositionen generell zu verwerfen. Andere dagegen sehen darin kein grundsätzliches Problem, sondern versuchen stattdessen, diese Verwendungen als Sonderfälle zu analysieren. Informative Präsuppositionen werden hierbei typischerweise unter dem Begriff der Akkommodation behandelt. Lewis $(1979,340)$ schreibt hierzu:

„If at time $\mathrm{t}$ something is said that requires presupposition $\mathrm{P}$ to be acceptable, and

if $\mathrm{P}$ is not presupposed just before $\mathrm{t}$, then - ceteris paribus and within certain limits - presupposition P comes into existence at t."

Führt eine präsupponierte Proposition eine neue Information ein, fügen die Diskursteilnehmer diese demnach gewöhnlich ohne umständingen Grounding-Prozess dem Common Ground hinzu. Zu klären ist dabei, welches die Bedingungen sind, unter denen eine Präsupposition akkommodiert werden kann.

Um den pragmatischen Begriff der Sprecherpräsupposition trotz der genannten Gegenbeispiele aufrechtzuerhalten, verändert u.a. Stalnaker (1974) den Gegebenheitsbegriff dahingehend, dass eine Präsupposition gegeben sein muss oder zumindest von S als gegeben dargestellt wird. Bezogen auf Beispiel (149) bedeutet dies, dass die präsupponierte Proposition $S$ hat geraucht von $\mathrm{S}$ als gegeben dargestellt wird, auch wenn sie noch nicht zum Wissen von A gehört. Eine solches Vorgehen ist vor 
allem dann möglich, wenn die Information nebensächlich und zu einem gewissen Grad erwartbar ist und demnach ein umständlicher Grounding-Prozess umgangen werden kann.

Die pragmatische Präsuppositionstheorie muss in der Lage sein, vorherzusagen, unter welchen Umständen die Akkommodation einer Präsupposition möglich ist und wann nicht. Untersuchungen zu diesem Punkt liegen insbesondere für die Verwendung von gebridgten NPn vor. Die Diskursanbindung von satzwertigen Präsuppositionen ist dagegen weniger gut untersucht und soll am Beispiel von temporalen Adverbialsätzen in Teil III diskutiert werden.

Stimmt der Common Ground-Ansatz, d.h., drücken Präsuppositionen tatsächlich eine gegebene Information aus oder müssen andernfalls akkommodiert werden, sollten sich Beispiele konstruieren lassen, in denen Akkommodation nicht möglich ist. Hierzu führt von Fintel (2000) den Präsuppositionauslöser too an, der nie akkommodiert werden kann. So kann in dem folgenden Beispiel die durch too ausgelöste Präsupposition nicht verarbeitet werden, wenn sie sich nicht auf eine bereits bekannte Information im Common Ground bezieht:

John can't come to the meeting tonight. He is having dinner in New York, too.

Als Kritikpunkt könnte man an dieser Stelle anführen, dass es sich bei too, oder analog dazu beim deutschen auch, um einen Ausdruck handelt, der semantisch unterspezifiziert ist und aus diesem Grund ähnlich wie Pronomen nie akkommodiert werden kann. Andere Präsuppositionen, wie beispielsweise faktive Komplementsätze, verfügen dagegen über einen reichen semantischen Inhalt, so dass Akkommodation meist möglich ist. Die Frage ist nun, ob sich auch für solche Präsuppositionen Kontexte finden lassen, in denen eine Akkommodation unmöglich ist. Bekannt sind die Beispiele, in denen der Gebrauch einer definiten NP ohne Antezedens ausgeschlossen ist. In dem folgenden Beispiel kann keine Relation zwischen dem Arzt und dem Diskurskontext hergestellt werden, weswegen der zweite Satz in diesem Zusammenhang keine wohlgeformte Äußerung bildet.

(155) Ich war gestern auf einer Hochzeit. \#Der Arzt trug einen gelben Anzug.

Auch in dem folgenden Beispiel kann die durch den Spaltsatz ausgelöste Präsupposition Jemand hat den Kuchen aufgegessen ohne weiteren Kontext nicht akkommodiert werden.

A: Was ist passiert?

B: \#Es war Klaus, der den Kuchen aufgegessen hat.

Die Beispiele zeigen, dass Akkommodation nicht immer möglich ist. Das spricht dafür, dass Präsuppositionen tatsächlich einem Common Ground-Constraint unterliegen. Die Fälle, in denen sie informativ sind, d.h. neue Informationen einführen, unterliegen strengen Bedingungen. Der Prozess der Akkommodation ist demnach keineswegs eine Reparaturstrategie, die in allen Fällen herangezogen werden kann, 


\section{Präsupponiertheit und At-issueness}

in denen eine scheinbare Ausnahme zur Theorie vorliegt. Im Gegenteil - eine ausgereifte Akkommodationstheorie sollte in der Lage sein, die Fälle vorherzusagen, in denen die Verwendung eines Präsuppositionsauslösers zu Unregelmäßigkeiten führt. Die Beobachtung, dass Präsuppositionen bereits im Common Ground gegeben sind, eignet sich nur bedingt zur Abgrenzung von [-at-issue]-Inhalten. Wir können zumindest festhalten, dass Inferenzen, die wie appositive Relativsätze nicht vorerwähnt sein können, eindeutig nicht präsupponiert sind. Dies ist im Einklang mit der Annahme, dass nur eine Untermenge der [-at-issue]-Inferenzen präsupponiert ist. Umgekehrt kann aber nicht geschlossen werden, dass nur Sätze, die eine bereits gegebene Information ausdrücken, präsupponiert sind. Als Kriterium zur Ermittlung von Präsuppositionen ist die pragmatische Anforderung an den Common Ground demnach nicht präzise genug, da zahlreiche Fälle von informativen Präsuppositionen, deren Inhalt akkommodiert werden muss, vorliegen. Um dennoch zwischen Präsuppositionen und nicht-präsupponierten Propositionen unterscheiden zu können, ist es wichtig, die dem Prozess der Akkommodation zugrundeliegenden Regeln herauszuarbeiten. Pragmatische Ansätze sind da häufig nicht präzise genug, indem sie davon ausgehen, dass erwartbare, unkontroverse Informationen einfach akkommodiert werden (so beispielsweise Lewis 1979). Eine genaue Ausbuchstabierung der Regeln, welche die Akkommodation von Präsuppositionen restringieren, wird erst in der anaphorischen Präsuppositionstheorie geleistet, welche im Folgenden diskutiert wird.

\subsubsection{Präsuppositionen sind anaphorisch}

Die Grundidee des pragmatischen Ansatzes besteht darin, dass Präsuppositionen Informationen ausdrücken, die zum Äußerungszeitpunkt bereits im Common Ground enthalten sind. Präsuppositionen stellen demnach Anforderungen an den Kontext. Diese Beobachtung wird in anaphorischen Präsuppositionstheorien weitergeführt, indem davon ausgegangen wird, dass Präsuppositionen Anaphern sind, die durch ein Antezedens gebunden werden müssen. Aus der eher abstrakten Gegebenheit im Common Ground wird in anaphorischen Ansätzen also eine konkrete Vorerwähntheit in Form eines Antezedens im Diskurskontext. Dadurch lassen sich die der Akkommodation zugrundeliegenden Regeln besser formalisieren und auf diese Weise kann besser zwischen präsupponierten und nicht-präsupponierten Inhalten unterschieden werden, wie im Folgenden gezeigt wird.

In Abschnitt 4.1.3.1 werden zunächst grundlegende Prinzipien der dynamischen Semantik als Voraussetzung für die anaphorische Betrachtung von Präsuppositionen erläutert. Wesentlich ist dabei insbesondere die semantische Beschreibung von indefiniten und definiten NPn im Rahmen der Diskurstheorien Discourse Representation Theory (kurz: DRT) und File Change Semantics (kurz: FCS) nach Heim (1982), Kamp (1981) und Kamp/Reyle (1993). Die in DRT und FCS für definite NPn vorgeschlagene anaphorische Analyse wird von Geurts (1999) und Van der Sandt (1992) auf alle Präsuppositionsauslöser übertragen und von Asher/Lascarides (1998b) und Asher/Lascarides (2003) im Rahmen der Segmented Discourse Repre- 
sentation Theory um Diskursrelationen erweitert, was Gegenstand der Abschnitte 4.1.3.2 und 4.1.3.3 ist.

\subsubsection{Hintergrund: Dynamische Semantik}

Die Wurzeln von anaphorischen Präsuppositionstheorien nach u.a. Geurts (1999) und Van der Sandt (1992) reichen zurück zu den diskurssemantischen Untersuchungen von definiten NPn durch Heim (1982) und Kamp (1981). Trotz unterschiedlicher Modelle, FCS bei Heim (1982) und DRT bei Kamp (1981), haben beide Untersuchungen gemeinsam, dass sie eine dynamische anstatt einer statischen Sicht auf Bedeutung voraussetzen. Neben wahrheitskonditionalen Bedeutungsaspekten werden so auch Phänomene an der Semantik-Pragmatik-Schnittstelle miteinbezogen (für einen Überblick über dynamische Bedeutungstheorien vgl. auch Chierchia 1995 und de Swart 1998). In beiden Theorien wird die Perspektive der Hörerin eingenommen, die den hereinkommenden Diskurs schrittweise verarbeitet. Jede Äußerung wird dabei immer vor dem Hintergrund des Diskurskontexts interpretiert. Eine Äußerung aktualisiert den Kontext, beispielsweise, indem die mit einer gemachten Assertion nicht kompatiblen Welten entfernt werden. Dieser Bedeutungsaspekt von Ausdrücken wird als ihr context change potential bezeichnet. Nach Stalnaker (1978) verfügt jeder Ausdruck über ein spezifisches Potential, den Kontext, in dem er verwendet wird, zu verändern. Ein Ausdruck $\phi$ bildet somit eine Update-Funktion $+\phi$, die das jeweilige Kontextset c als Argument nimmt und es auf ein neues Kontextset c' abbildet. Der auf diese Weise aktualisierte Kontext bildet dann wieder den Hintergrund für die darauffolgenden Äußerungen.

Sowohl in FCS als auch in DRT wird die semantische Repräsentation um eine zusätzliche Ebene erweitert, die sogenannten files in FCS beziehungsweise Diskursrepresentationsboxen in DRT. Es handelt sich dabei um ein partielles Modell des soweit verarbeiteten Diskurses, welches ständig aktualisiert wird. Für Entitäten, die durch den Text eingeführt werden, stehen Diskursreferenten. Im Rahmen von FCS wird für jeden neu eingeführten Diskursreferenten eine neue file card eröffnet, die alle Informationen über diesen Referenten sammelt. In DRT dagegen entspricht eine Diskursrepräsentationsstruktur, dargestellt als Box, einem Diskurs. Sie besteht aus einem Diskursuniversum, das alle Diskursreferenten enthält, sowie Diskurskonditionen, die den Informationen über diese Referenten entsprechen.

Beide Untersuchungen der Semantik von NPn kommen innerhalb unterschiedlicher theoretischer Modelle zu dem Schluss, dass indefinite NPn einen neuen Diskursreferenten einführen, definite dagegen nicht. Anders als in der Russellschen Kennzeichnungstheorie (siehe (145) in Abschnitt 4.1.1) werden NPn nicht als referierende Ausdrücke, sondern vielmehr als Variablen, die es ermöglichen, einen Diskursreferenten über die Satzgrenze hinaus im Blick zu behalten, analysiert. Betrachten wir hierzu ein Beispiel. In dem folgenden Satz führt die indefinite NP ein Mann einen neuen Diskursreferenten ein, der durch die definite NP im zweiten Satz aufgegriffen wird. Je nach Kontext kann sich die definite NP auch auf ein anderes Antezedens j beziehen. 
$[\text { Ein Mann }]_{i}$ kam in die Kneipe. [Der Mann $]_{i / j}$ setzte sich an den Tresen.

Wird die Reihenfolge der beiden NPn dagegen vertauscht, können sie nicht mehr koreferent sein. Die definite NP im ersten Satz bezieht sich auf ein explizit nicht genanntes Antezedens, wohingegen die indefinite NP im zweiten Satz einen neuen Diskursreferenten einführt:

(158) [Der Mann $]_{j}$ kam in die Kneipe. [Ein Mann $]_{i / * j}$ setzte sich an den Tresen.

Die Beobachtung, dass indefinite NPn einen neuen Diskursreferenten einführen, wohingegen definite einen bereits bekannten aufgreifen, fasst Heim (1982, 369f) wie folgt zusammen zusammen:

\section{Extended-Novelty-Familiarity Condition:}

For $\phi$ to be felicitous wrt $\mathrm{F}$ it is required for every $\mathrm{NP}_{i}$ in $\phi$ that:

(i) if $\mathrm{NP}_{i}$ is $[-$ definite], then $\mathrm{i} \notin \operatorname{Dom}(\mathrm{F})$, and

(ii) if $\mathrm{NP}_{i}$ is [+definite], then $\mathrm{i} \in \operatorname{Dom}(\mathrm{F})$, and $\mathrm{F}$ entails $\mathrm{NP}_{i}$

Im Rahmen von FCS enthält die Domäne $\operatorname{Dom}(\mathrm{F})$ alle aktiven Indizes eines Diskurses. Die Regel besagt nun, dass eine indefinite NP einen neuen Index einführt, während der Index einer definiten NP einer bereits aktiven File-Karte in $\operatorname{Dom}(\mathrm{F})$ zugeordnet werden muss. Der semantische Inhalt einer definiten NP muss sich zudem als Implikation aus dem Common Ground ergeben. Letzteres erinnert an die Darstellung von Präsuppositionen in Common-Ground-Theorien.

Die Modellierung von definiten und indefiniten NPn innerhalb von DRT ist sehr ähnlich. Auch in DRT wird die Perspektive der Hörerin eingenommen, die den Sprachfluss als Input verarbeitet. Die in DRT verwendete Darstellung des durch die Hörerin verarbeiteten Outputs entspricht Boxen, welche als Diskursrepräsentationsstrukturen (kurz: DRS) bezeichnet werden. Diese werden durch den fortschreitenden Diskurs ständig erweitert. Eine DRS enthält dabei zum einen ein sogenanntes Universum von Diskursreferenten, die den Objekten, um die es geht, entsprechen, und zum anderen Konditionen, die den deskriptiven Inhalt der Prädikate enthalten. Ein Satz wie der folgende erhält so die Struktur in (161):

(160) Ein Linguist krault eine Katze.

\begin{tabular}{|l|}
\hline $\mathrm{x} y$ \\
\hline Linguist $(\mathrm{x})$ \\
Katze $(\mathrm{y})$ \\
kraulen $(\mathrm{x}, \mathrm{y})$ \\
\hline
\end{tabular}

Das Diskursuniversum enthält zwei Referenten, x und y, zudem besitzt die DRS drei Konditionen. Wird der aus einem Satz bestehende Diskurs in (160) nun um einen weiteren Satz erweitert, bildet der bereits verarbeitete Satz (160) den Hintergrund 
für die folgende Äußerung in (162). Statt einer definiten NP wird hier der Einfachheit halber ein Pronomen verwendet. Die genaue Modellierung von definiten NPn unterscheidet sich nur geringfügig von Pronomen und wird im folgenden Kapitel genauer erläutert.

Sie beißt ihn.

Die folgende DRS gibt Satz (162) isoliert vom Diskurskontext und vor der Anaphernresolution wieder:

$$
\begin{array}{|l|}
\hline \mathrm{v} w \\
\hline \text { beißen }(\mathrm{v}, \mathrm{w}) \\
\hline
\end{array}
$$

In einem nächsten Schritt wird Satz (162) in den vorherigen Diskurskontext, welcher aus Satz (160) besteht, integriert. Dabei findet ein Update des bisherigen Diskurses durch den hereinkommenden Satz statt, was einer Vereinigung der beiden Diskursmengen entspricht. Nun werden auch die Anaphern aufgelöst, indem v mit y und w mit x gleichgesetzt werden. Kommen mehrere Diskursreferenten als mögliche Antezedentia in Frage, spielen diverse linguistische und nicht-linguistische Faktoren bei der Anaphernresolution eine Rolle. In dem vorliegenden Beispiel jedoch ist die Zuordnung der Antezedentia aufgrund der Genusunterscheidung eindeutig. Der resultierende Diskurs ist in (164) dargestellt:

\begin{tabular}{|l|}
\hline $\mathrm{x} y \mathrm{v} \mathrm{w}$ \\
\hline Linguist(x) \\
Katze $(\mathrm{y})$ \\
kraulen $(\mathrm{x}, \mathrm{y})$ \\
beißen $(\mathrm{v}, \mathrm{w})$ \\
$\mathrm{v}=\mathrm{y}$ \\
$\mathrm{w}=\mathrm{x}$ \\
\hline
\end{tabular}

Zusammenfassend kann festgehalten werden, dass indefinite NPn wie in Beispiel (160) einen neuen Diskursreferenten ins Diskursuniversum einführen, wohingegen Pronomen und definite NPn als anaphorische Ausdrücke durch einen bereits präsenten Referenten, welcher als Antezedens fungiert, gebunden werden müssen.

Weder Heim (1982) noch Kamp (1981) haben den Anspruch, eine Präsuppositionstheorie zu liefern, sondern beschränken sich lediglich auf die diskurssemantische Untersuchung von NPn. Definite NPn sind jedoch auch immer Präsuppositionsauslöser. So bemerkt auch Heim (1982, 230ff), dass der deskriptive Inhalt von definiten Ausdrücken immer präsupponiert ist, während der von indefiniten Ausdrücken assertiert wird. Im Rahmen der hier vorgestellten Theorie bedeutet das, dass der präsupponier- 


\section{Präsupponiertheit und At-issueness}

te Inhalt von definiten Ausdrücken bereits in der Diskursrepräsentation gegeben ist, wohingegen indefinite Ausdrücke den Kontext um ihren semantischen Inhalt erweitern. Präsuppositionen sind demnach auch im Rahmen von anaphorischen Ansätzen Anforderungen an den Kontext: Ein Satz, der eine definite NP enthält, kann nur dann interpretiert werden, wenn diese durch ein Antezedens im Kontext gebunden werden kann, das heißt, wenn der Kontext so beschaffen ist, dass er die Präsupposition impliziert. Dies ist analog zu der von Stalnaker (1974) geprägten Sicht auf Präsuppositionen als Inferenzen, die im Common Ground enthalten sind. Es ist demnach nur eine logische Konsequenz, die Analyse von definiten NPn auf die Menge aller Präsuppositionsauslöser auszuweiten. Genau dies unternehmen Geurts (1999) und Van der Sandt (1992) in der Bindungstheorie von Präsuppositionen. ${ }^{32}$

\subsubsection{Die Bindungstheorie von Präsuppositionen}

Van der Sandt (1992) beobachtet zahlreiche Ähnlichkeiten im Verhalten von traditionell als Anaphern bezeichneten Ausdrücken und Präsuppositionen und argumentiert dafür, dass es sich bei Präsuppositionen um eine spezielle Unterart von Anaphern handelt. Die im Rahmen von DRT und FCS entwickelte Sichtweise auf definite NPn weitet Van der Sandt (1992) auf alle Präsuppositionsauslöser aus.

Nach Van der Sandt (1992) sind Präsuppositionen anaphorische Ausdrücke, die, anders als Pronomen, semantischen Inhalt besitzen. Aus diesem Grund können Präsuppositionen nicht nur wie gängige Anaphern durch ein Antezedens im Kontext gebunden, sondern auch akkommodiert werden, wenn kein Antezedens vorhanden ist. Beide Prozesse, Bindung und Akkommodation von Präsuppositionen, modelliert Van der Sandt (1992) im Rahmen von DRT. Ein Überblick über die Bindungstheore von Präsuppositionen findet sich in u.a. Asher/Lascarides (1998b), Geurts (1999), Lascarides/Oberlander (1993), Schilder (1999) und Van der Sandt/Geurts (1991). ${ }^{33}$ Präsuppositionen unterscheiden sich nun allerdings in zwei Aspekten von anderen anaphorischen Ausdrücken, was auch der Grund dafür ist, sie als einen spezifischen Untertyp der Anaphern zu betrachten. Zum einen besitzen Präsuppositionen anders als pronominale Ausdrücke eigenen semantischen Inhalt. Aus diesem Grund können Präsuppositionen akkommodiert werden, pronominale Anaphern hingegen nicht. ${ }^{34}$

${ }^{32}$ Die im folgenden Abschnitt dargestellte Bindungstheorie von Präsuppositionen bildet eine Weiterentwicklung von DRT. Eine Implementierung in FCS nach Heim (1982) wäre aber ebenso denkbar, da es sich bei den Unterschieden zwischen den beiden Modellen hauptsächlich um Notationsunterschiede handelt.

${ }^{33}$ Der Begriff der Bindung ist hier nicht syntaktisch zu verstehen, sondern referiert auf die rein semantische Gleichsetzung eines anaphorischen Ausdrucks mit einem koreferenten Antezedens.

${ }^{34}$ In der Tradition von Lewis (1979) modelliert auch Van der Sandt (1992) Akkommodation als reine Hinzufügung der präsupponierten Inhalte auf einer Diskursebene. Die Möglichkeit der Akkommodation unterliegt dabei spezifischen Bedingungen, die von Van der Sandt (1992) im Rahmen von DRT als Akzessibilitätsbeschränkungen modelliert werden. Je nachdem, auf welcher Diskursebene der präsupponierte Referent hinzugefügt wird, unterscheidet man lokale von globaler Akkommodation (für Details vgl. Van der Sandt 1992). Neben Akzessibilitätsbeschränkungen wird Akkommodation auch durch nicht-strukturelle Faktoren wie Salienz, Neuheit oder nicht-linguistisches Wissen geregelt. 
Der zweite wesentliche Unterschied zwischen Präsuppositionen und pronominalen Anaphern besteht darin, dass Präsuppositionen über eine eigene, zum Teil komplexe interne Struktur verfügen, ein Punkt, der natürlich mit ihrem semantischen Inhalt zusammenhängt. So können Präsuppositionen prinzipiell dieselbe innere Komplexität aufweisen wie Assertionen, indem sie Variablen oder weitere Präsuppositionen enthalten. Dies ist in dem folgenden Beispielsatz, frei adaptiert nach Van der Sandt (1992, 353), gegeben, in dem innerhalb des präsupponierten Komplementsatzes weitere Präsuppositionsauslöser eingebettet sind.

(165) Evi weiß, dass es Jenny war, die die Pralinen aufgegessen hat.

Die Bindung beziehungsweise Akkommodation von Präsupposition modellieren Geurts (1999), Van der Sandt/Geurts (1991) und Van der Sandt (1992) innerhalb der Diskurstheorie DRT. Zentral ist dabei, dass Präsuppositionsauslöser wie beispielsweise definite NPn oder faktive Verben eine eigene DRS, eine sogenannte $\alpha$-DRS, einführen. Präsupponierte Inhalte werden also getrennt von assertierten verarbeitet (vgl. für einen ähnlichen Ansatz auch die Layered DRT von Geurts/Maier 2003). Betrachten wir dazu ein Beispiel. Die definite NP im zweiten Satz von Beispiel (166) führt als Präsuppositionsauslöser eine $\alpha$-DRS ein, die in (167) vereinfacht dargestellt ist.

(166) Peter krault eine Katze. Die Katze schnurrt.

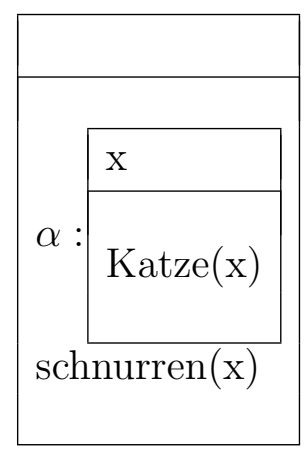

Wie ein gewöhnliches Pronomen muss der Inhalt innerhalb einer solchen $\alpha$-DRS im Normalfall gebunden werden. Dazu wird die entsprechende $\alpha$-DRS in den jeweils relevanten Diskurskontext inkorporiert, welcher bezogen auf unser Beispiel aus dem ersten Satz von Beispiel (166) besteht. Dies ist in der linken Struktur von (168) dargestellt. Anschließend muss die anaphorische Relation aufgelöst werden, wodurch wir die rechte Struktur in (168) erhalten. ${ }^{35}$

\footnotetext{
${ }^{35}$ Auch Eigennamen führen als Präsuppositionauslöser eine separate $\alpha$-DRS ein. Der Einfachheit halber werden die durch Eigennamen ausgelösten Präsuppositionen im Folgenden ignoriert.
} 


\begin{tabular}{|l|}
\hline $\mathrm{p} \mathrm{y}$ \\
\hline Peter $(\mathrm{p})$ \\
Katze $(\mathrm{y})$ \\
kraulen $(\mathrm{p}, \mathrm{y})$ \\
$\mathrm{x}$ \\
$\alpha: \operatorname{Katze}(\mathrm{x})$ \\
\hline schnurren $(\mathrm{x})$ \\
\hline
\end{tabular}

\begin{tabular}{|l|}
\hline $\mathrm{p} \mathrm{y} \mathrm{x}$ \\
\hline Peter $(p)$ \\
Katze(y) \\
kraulen(p,y) \\
Katze $(x)$ \\
schnurren $(x)$ \\
$\mathrm{y}=\mathrm{x}$ \\
\hline
\end{tabular}

Erst wenn alle $\alpha$-Strukturen aufgelöst sind, ist eine semantische Interpretation des Satzes möglich. Ist Bindung, also die Gleichsetzung der Präsupposition mit einem anderen Diskursreferenten mit demselben Inhalt, nicht möglich, muss die Präsupposition akkommodiert werden. Dies bedeutet, dass der präsupponierte Diskursreferent einfach auf einer Diskursebene ohne Anbindung hinzugefügt wird. Bindung hat dabei immer Priorität vor Akkommodation (vgl. Van der Sandt 1992, 362).

Zentral ist nun, dass nicht nur NPn, sondern auch CPn Variablen für Diskursreferenten sind: Während NPn Variablen für Entitäten, welche im Folgenden mit den Buchstaben x, y, v etc. dargestellt werden, einführen, sind CPn Variablen für Ereignisse, auf die ich ähnlich wie in SDRT im Folgenden mit $\pi_{1}, \pi_{2}$ etc. verweisen werde. Auch für CPn gilt wie für NPn, dass sie entweder einen neuen oder einen bereits gegebenen Diskursreferenten einführen können. Während Assertionen typischerweise einen neuen Referenten einführen, generiert eine satzwertige Präsuppositionen dagegen wie eine definite NP eine eigene $\alpha$-Struktur und muss entweder mit einem Antezedens gleichgesetzt oder aber akkommodiert werden. Betrachten wir dazu das folgende Beispiel. Hier wird durch den ersten Satz ein Ereignis $\pi_{1}$ (= Peter hat geraucht) eingeführt. Der 2. Satz führt zwei Variablen ein: $\pi_{2}$ (= Peter bedauert, dass er geraucht hat) und $\pi_{3}$ (= Peter hat geraucht), wobei $\pi_{3}$ eine separate AlphaStruktur eröffnet genau wie eine definite NP.

Peter hat geraucht. Er bedauert, dass er geraucht hat.

Genau wie in (168) wird nun $\pi_{3}$ durch $\pi_{1}$ gebunden, also mit der Operation ,=' gleichgesetzt. Obgleich Bindung nach Van der Sandt (1992) bei der Interpretation von Präsuppositionen den Standardfall darstellt, wirkt der Diskurs (169) redundant. Dies hängt damit zusammen, dass satzwertige Präsuppositionen aufgrund ihres reichen semantischen Inhalts leicht akkommodiert werden können. Nun trachten Sprecher aber immer danach, die stärkst mögliche Präsupposition zu wählen, um Redundanz und übertriebene Explizitheit zu vermeiden, was Heim (1991) in dem Prinzip Maximize Presupposition zusammenfasst. Die Stärke eines Ausdrucks bezogen auf das Prinzip Maximize Presupposition bemisst sich dabei nach den zwei folgenden Kriterien: 
(170) a. Ein präsupponierter Ausdruck ist stärker als ein nicht-präsupponierter Ausdruck.

b. Ein präsupponierter Ausdruck $\mathrm{p}_{1}$ ist stärker als ein präsupponierter Ausdruck $\mathrm{p}_{2}$, wenn $\mathrm{p}_{1}$ weniger semantischen Inhalt besitzt als $\mathrm{p}_{2}$.

Aus diesem Grund scheint für satzwertige Präsuppositionen eventuell sogar die Akkommodation den Standardfall darzustellen. In diesem Fall würde der nachgestellte Satz aus Beispiel (169) ohne den ersten Satz geäußert werden und der durch den präsupponierten Satz eingeführte Diskursreferent müsste auf eine Ebene des Diskursmodells hinzugefügt werden. Wie häufig die Verwendung von präsupponierten Nebensätzen ohne Vorerwähnung tatsächlich ist, bedarf noch einer genauen empirischen Überprüfung.

Wir können festhalten, dass Präsuppositionen von Geurts (1999) und Van der Sandt (1992) als anaphorische Ausdrücke behandelt werden, die anders verarbeitet werden als assertierte Inhalte. Jede Präsupposition führt eine separate Struktur ein und wird bei ihrer Eingliederung in den Diskurs entweder durch ein Antezedens gebunden oder aber an einer zugänglichen Stelle des Diskurskontexts hinzugefügt, d.h. akkommodiert. Neu ist dabei insbesondere, dass satzwertige Präsuppositionen genauso analysiert werden wie definite NPn.

\subsubsection{Präsuppositionen in SDRT}

An der Bindungstheorie von Präsuppositionen, wie sie Geurts (1999) und Van der Sandt (1992) im Rahmen von DRT modellieren, kritisieren Asher/Lascarides (1998b) und Asher/Lascarides (2003), dass die Interpretation von Präsuppositionen rein strukturellen Beschränkungen gehorcht. Zusätzlich zu Kontext- und Weltwissen führen Asher/Lascarides (1998b), Asher/Lascarides (2003) und Lascarides/Oberlander (1993) als wesentlichen Parameter bei der Verarbeitung von Anaphern die rhetorische Funktion des jeweiligen Satzes in Bezug auf den Diskurskontext an. Zu diesem Zweck modellieren sie die Verarbeitung von Präsuppositionen innerhalb von SDRT. Anders als DRT berücksichtigt SDRT als Formalisierung an der Semantik-PragmatikSchnittstelle pragmatische Faktoren, die in die Formalisierung von DRT keinen Eingang gefunden haben. Wesentlich dabei sind die zugrundeliegenden rhetorischen Relationen zwischen den einzelnen Diskursteilen. Auf diese Weise ist es möglich, bei der Verarbeitung von Präsuppositionen die Interaktion zahlreicher Faktoren abzubilden (für einen Überblick vgl. Asher/Lascarides 2003).

Der wesentliche Unterschied in der Behandlung von Präsuppositionen im Vergleich zu Van der Sandt (1992) betrifft die Art der Anbindung an den Diskurs. Wie weiter oben dargestellt, werden in der Theorie von Van der Sandt (1992) Präsuppositionen entweder durch ein identisches Antezdens gebunden oder aber akkommodiert. Der von Lewis (1979) begründeten Sicht folgend, bezeichnet Akkommodation dabei die einfache Hinzufügung des präsupponierten Inhalts auf einer Diskursebene. Im Rahmen von SDRT dagegen wird die Interpretation von Präsuppositionen als Prozess der Diskursanbindung betrachtet, bei dem der präsupponierte Inhalt durch 
eine rhetorische Relation mit dem Kontext verknüpft wird. Dabei ist die Art der Diskursrelation grammatisch nicht vorgegeben. Der Vorteil eines solchen Verfahrens im Vergleich zur einfachen Bindung bzw. Akkommodation zeigt sich bei der Betrachtung des folgenden Beispiels. Die durch auch ausgelöste Präsupposition kann kontextunabhängig nicht klar definiert werden. Sie kann, wie in (171-a) und (171-b) dargestellt, entweder lauten, dass Jap noch an einem anderen Ort außer Paris war, oder aber, dass außer Jap noch jemand in Paris war.

(171) Jap war auch in Paris.

a. \Jap war in Rom.

b. \Evi war in Paris.

Da unklar ist, wie die durch auch ausgelöste Präsupposition lautet, kann diese auch nicht einfach lokal oder global hinzugefügt, also akkommodiert werden. Stattdessen muss im vorherigen Kontext ein (indirektes) Antezedens wie (171-a) oder (171-b) gegeben sein, an das die Präsupposition durch eine zweistellige Diskursrelation wie etwa $\mathrm{R}=$ Parallel angebunden wird. Um eine Präsupposition zu interpretieren, bedarf es demnach mehr als der reinen Hinzufügung bzw. Bindung wie bei Van der Sandt (1992). Da Präsuppositionen anaphorisch sind, müssen sie immer mit einem Teil des Kontexts verbunden werden. Bei der Verarbeitung von Präsuppositionen sind demnach zwei Formen von semantischer Unbestimmtheit aufzulösen: Eine Präsupposition wird immer über eine unspezifizierte Diskursrelation $\mathrm{R}$ an einen unspezifizierten Teil des Kontexts angebunden. Beide Formen der Unbestimmtheit sind in (172) nach Asher/Lascarides (2003, 173) dargestellt und müssen bei der Interpretation der Präsupposition aufgelöst und angereichert werden. Dabei muss von $\mathrm{H}$ herausgearbeitet werden, welche rhetorische Relation $\mathrm{R}$ geeignet ist, und welcher Diskursteil $\pi_{1}$ beziehungsweise $\pi_{2}$ als Anknüpfungsort für die Präsupposition infrage kommt. Nach Asher/Lascarides (2003) kann die Präsupposition $\pi_{p}$ dabei, wie in (172) dargestellt, erstes oder zweites Argument von R sein.

$$
\begin{aligned}
& \mathrm{R}(\mathrm{u}, \mathrm{v}), \mathrm{R}=?, \mathrm{u}=?, \mathrm{v}=? \\
& \left(\pi_{p}=\mathrm{u} \vee \pi_{p}=\mathrm{v}\right)
\end{aligned}
$$

Wie pronominale Anaphern sind folglich auch Präsuppositionen semantisch unterspezifiziert. Die Resolution der beiden Formen von Unbestimmtheit führt schließlich zu einer Definition des semantischen Skopus der Präsupposition.

Die Struktur eines Satzes mit einer sententialen Proposition soll kurz an einem Beispiel erläutert werden. Satz (173) enthält eine Assertion, welche dem Gesamtsatz entspricht, und einen präsupponierten Komplementsatz.

(173) Jenny bedauerte, dass Laura krank war.

In (174) ist zu sehen, dass präsupponierter und assertierter Inhalt wie auch in der Theorie von Van der Sandt (1992) getrennt voneinander verarbeitet werden, dargestellt als zwei SDRT-Strukturen a und p. Zentral ist nun, dass innerhalb der 
präsupponierten Struktur p eine unterspezifizierte rhetorische Relation $\mathrm{R}$, welche zwei unterspezifizierte Variablen u und v miteinander verknüpft, generiert wird. Dabei entspricht die Präsupposition entweder dem ersten Argument u oder dem zweiten Argument v von R (vgl. Asher/Lascarides 2003, 176). ${ }^{36}$
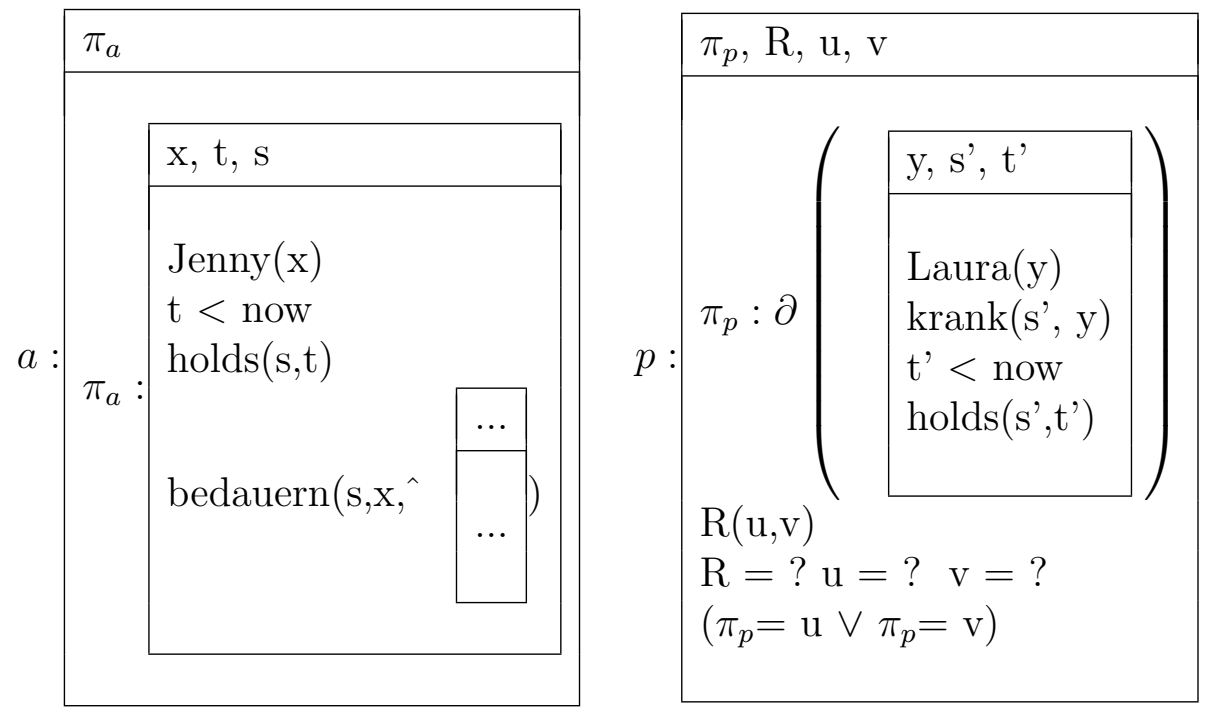

Um die Äußerung interpretieren zu können, muss der Wert von $\mathrm{R}$ determiniert werden, außerdem ist festzulegen, an welches Antezedens die Präsupposition angebunden wird und ob die Präsupposition dabei dem ersten oder dem zweiten Argument von R entspricht. Wie eine definite NP kann die Präsupposition durch einen bereits im Diskursuniversum gegebenen Diskursreferenten gebunden werden. Dies ist beispielsweise der Fall, wenn Satz (173) in dem folgenden Kontext geäußert wird.

(175) Laura war krank. Jenny bedauerte, dass sie krank war.

Hier wird nun durch den ersten Satz ein Referent $\pi_{1}$ (= Laura war krank) eingeführt, durch den zweiten Satz ein Referent $\pi_{2 a}$ (= Jenny bedauerte, dass Laura krank war) und durch die eingebettete Proposition ein Referent $\pi_{2 p}$ (= Laura war krank). Der Diskursreferent $\pi_{2 p}$ wird mit $\pi_{1}$ gleichgesetzt, da sich beide Referenten auf dasselbe Ereignis beziehen. Den Wert, welchen die Diskursrelation R bei Gleichsetzung zweier Diskursreferenten annimmt, wird im Folgenden als Coreference bezeichnet. In Beispiel (175) werden das unterspezifizierte $\mathrm{R}, \mathrm{u}$ und v nach der Merge-Operation demnach wie folgt aufgelöst: $\mathrm{R}(\mathrm{u}, \mathrm{v}), \mathrm{R}=$ Coreference, $\mathrm{u}=\pi_{1}$ und $\mathrm{v}=\pi_{2 p}$. Aufgrund des Prinzips Maximise Presupposition ist jedoch zu erwarten, dass das Eintreten dieses Falls beschränkt wird. So ist es zur Redundanzvermeidung sinnvoll, einen stärkeren Präsuppositionsausdruck zu wählen als die satzwertige Präsupposition in (175). Eine stärkere Präsupposition würde hier beispielsweise das Pronomen das bilden, da es weniger semantischen Inhalt besitzt als der faktive Komplementsatz in (175).

\footnotetext{
${ }^{36} \mathrm{Im}$ Folgenden wird wie bei Asher/Lascarides (2003) der Operator $\delta$ anstatt des $\alpha$-Operators von Van der Sandt (1992) verwendet, um präsupponierte Inhalte als solche zu markieren.
} 


\section{Präsupponiertheit und At-issueness}

Eine Präsupposition kann auch über eine andere Diskursrelation als Coreference angebunden werden. In der folgenden Äußerung drückt der Komplementsatz beispielsweise ein Unterereignis des ersten Satzes aus. In diesem Fall wird der Komplementsatz über $\mathrm{R}=S u b$-Part mit einem indirekten Antezedens verknüpft.

Bei der Schneewanderung hatte sich die ganze Klasse erkältet. Jenny bedauerte, dass Laura krank war.

Dass eine sententiale Präsupposition auch über eine Diskursrelation an ein indirektes Antezedens angebunden werden kann, ist analog zu der Beschreibung von gebridgten NPn, die ebenfalls mithilfe einer bestimmten semantischen Relation an einen Teil des vorherigen Kontextes gebunden werden (vgl. Beispiel (151) in Abschnitt 4.1.2). So kann eine definite NP wie in Beispiel (177-a) über $\mathrm{R}=$ Coreference durch ein koreferentes Antezedens gebunden werden oder aber über eine Teil-Ganzes-Relation $\mathrm{R}=$ Sub-Part wie in Beispiel (177-b) mit einem indirekten Antezedens verknüpft werden.

a. Christina hat sich ein Auto gekauft. Das Auto ist pink.

b. Christina hat sich ein Auto gekauft. Der Motor war schon nach einer Woche kaputt.

Attraktiv an der Implementierung von Präsuppositionen in SDRT ist, dass keinerlei speziell auf Präsuppositionen zugeschnittene Extraannahmen benötigt werden. Stattdessen wird derselbe Prozess der Diskursanbindung mittels rhetorischer Relationen herangezogen, der auch bei der Verarbeitung von definiten NPn eine Rolle spielt. Von der Bindungstheorie nach Van der Sandt (1992) übernehmen Asher/Lascarides (1998b) und Asher/Lascarides (2003) die Annahme, dass es sich bei Präsuppositionen um anaphorische Ausdrücke handelt, welche durch ein Antezedens gebunden werden müssen. Während Präsuppositionen bei Van der Sandt (1992) jedoch entweder durch ein koreferentes Antezedens gebunden oder aber akkommodiert, d.h. dem Diskursuniversum einfach hinzugefügt werden, modellieren Asher/Lascarides (1998b) und Asher/Lascarides (2003) die Interpretation von Präsuppositionen immer als Diskursanbindung.

Van der Sandt (1992) weitet eine Analyse, die vorher nur für definite NPn angenommen wurde, auf alle Präsuppositionsauslöser aus, indem er zeigt, dass alle Präsuppositionen Anaphern sind. Ähnlich wird nun im Rahmen der Diskursanalyse SDRT ein anderer Aspekt, der zuvor nur im Hinblick auf definite NPn untersucht wurde, auf alle Präsuppositionen, aber auch auf nicht-initiale Assertionen übertragen. Nimmt man der Argumentation von Van der Sandt (1992) folgend an, dass alle Präsuppositionen wie definite NPn Anaphern sind und durch ein Antezedens gebunden werden müssen, ist es naheliegend, dass auch alle Präsuppositionen indirekt durch eine Diskursrelation gebunden werden können. Van der Sandt (1992) hat zwar die Analyse von definiten NPn auf andere Präsuppositionsauslöser übertragen, indem er annimmt, dass alle Präsuppositionen wie definite NPn ein Antezedens aufgreifen. Jedoch ist er nicht den logisch darauf aufbauenden Schritt gegangen, festzustellen, dass 
auch alle Präsuppositionen wie definite NPn indirekt über eine Bridging-Relation an den Diskurs angebunden werden können, wenn kein direktes, d.h. koreferentes, Antezedens vorhanden ist. Stattdessen modelliert Van der Sandt (1992) die Akkommodation von Präsuppositionen als reine Hinzufügung. Dieser Schritt wird nun in Asher/Lascarides (1998b), Asher/Lascarides (2003) und Lascarides/Oberlander (1993) vollzogen, indem Präsuppositionen durch eine rhetorische Relation an den Diskurs gebunden werden, so wie definite NPn durch eine Bridging-Relation interpretiert werden können. Die Anbindung präsupponierter Inhalte über eine Diskursrelation betrifft nun nicht nur solche Präsuppositionen, die in der Theorie von Van der Sandt (1992) akkommodiert werden müssten, sondern generell alle Präsuppositionen. Die in Van der Sandt (1992) gemachte Unterscheidung zwischen Bindung und Akkommodation wird im Rahmen von SDRT demnach nicht aufrechterhalten.

Die Verarbeitung von Präsuppositionen als einen Prozess der Diskursanbindung zu betrachten, restringiert u.a. auch den Akkommodationsprozess. Die Akkommodation einer Präsupposition ist im Rahmen von SDRT nur dann möglich, wenn der präsupponierte Inhalt durch eine rhetorische Relation mit einem vorherigen Äußerungsteil verknüpft werden kann. Ist dies nicht möglich, ist die Anbindung fehlgeschlagen und Akkommodation ausgeschlossen.

Inwiefern eignet sich die anaphorische Beschreibung von Präsuppositionen nun zur Abgrenzung von anderen [-at-issue]-Inferenzen? Anders als nicht-präsupponierte Inferenzen müssen Präsuppositionen immer über ein Antezedens im Diskurs lizenziert sein. Nicht-präsupponierte Nebensätze wie beispielsweise appositive Relativsätze können dagegen komplett vom sie einbettetenden Diskurs gelöst sein. Zudem unterscheiden sich präsupponierte von nicht-präsupponierten Inferenzen darin, mit welchen Diskursrelationen sie angebunden werden können. Hierbei kann folgende Regel formuliert werden:

\section{Coreference-Regel}

Ausdrücke, die eindeutig [-präsupponiert] sind, können nicht über die Relation $\mathrm{R}=$ Coreference mit einem Antezedens verknüpft werden.

Die Regel soll eine zentrale Beobachtung erfassen: Zu der im Rahmen von dynamischen Diskursmodellen wie DRT und FCS getroffenen Aussage, dass definite NPn immer ein koreferentes Antezedens aufgreifen, existieren zahlreiche Ausnahmen, die einer empirischen Studie von Schwarz (1998) zufolge sogar die Mehrheit der Verwendungen ausmachen. Umgekehrt gilt die Aussage, derzufolge indefinite NPn nie ein Antezedens aufgreifen, ausnahmslos. Beispielsweise ist es in keinem Kontext möglich, dass die indefinite NP in dem folgenden Diskurs mit der definiten koreferent ist. ${ }^{37}$

$$
\text { \#Christina hat sich das } \mathrm{Auto}_{i} \text { gekauft. Ein } \mathrm{Auto}_{i} \text { war schon nach einer }
$$

\footnotetext{
${ }^{37}$ Cunha Lima et al. (2011) nennen im Rahmen ihrer psycholinguistischen Studie Beispiele wie das folgende als Beweis dafür, dass auch indefinite NPn durch ein koreferentes Antezedens gebunden werden können:
}

$[\text { The pirate }]_{i}$ shot a monkey on the beach. $[\text { A pirate }]_{i}$ with a pistol. 


\section{Präsupponiertheit und At-issueness}

Woche kaputt.

Es ist demnach sinnvoll, bei der Beschreibung der Semantik von indefiniten und definiten NPn, bzw. von nicht-präsupponierten Inferenzen und Präsuppositionen, letztere nicht nur vor dem Hintergrund von Nicht-Präsupponiertem zu beschreiben. Vielmehr bietet es sich an, auch indefinite NPn und andere nicht-präsupponierte Inhalte über ihr Unvermögen, einen bereits gegebenen Diskursreferenten aufzugreifen, zu definieren. Bisherige Ansätze definieren lediglich Präsuppositionen über Auflagen: Sie müssen wahr sein und sie stellen Anforderungen an den Kontext, der sie implizieren muss. Zusätzlich wird mit (178) vorgeschlagen, auch die Verwendung von eindeutig nicht-präsupponierten Inferenzen über die Coreference-Regel zu beschränken. Diese Beobachtung ist jedoch nur bedingt als Präsuppositionstest geeignet, da die meisten Inferenzen eine Lesart als Präsupposition zulassen, wenn dies durch den Kontext forciert wird. ${ }^{38}$ Beispielsweise würde man nicht davon ausgehen, dass das Brückenverb glauben einen präsupponierten Satz einbettet, dennoch ist die folgende Konstruktion natürlich absolut wohlgeformt.

(180) Laura hat geraucht und Jenny glaubt, dass Laura geraucht hat.

Als Präsuppositionstest eignet sich die Coreference-Regel demnach nur für solche Inferenzen, welche wie indefinite NPn grundsätzlich nicht präsupponiert sein können. Dazu zählen appositive Relativsätze, wie das Beispiel (96-a) aus Holler (2005, 54) und Beispiel (181-a) belegen. Genausowenig kann ein durch einen V2-Nebensatz eingeführter Diskursreferent $\pi_{2}$ Argument einer Relation $\mathrm{R}=$ Coreference sein, weswegen die Beispiele (181-b) und (181-c) ungrammatisch sind. Dies deckt sich mit der weit verbreiteten Auffassung, derzufolge V2-Stellung nicht in präsupponierten Nebensätzen möglich ist (vgl. dazu die Diskussion in Teil II).

(181) a. Jap ist gerade in Indonesien. \#Jap, der gerade in Indonesien ist, fährt gerne ans Meer.

b. Oma hat gestern ein neues Fastnachtskostüm gekauft. \#Sie ist glücklich, weil sie hat ein neues Fastnachtskostüm gekauft.

c. Susanne hat gestern abgeschrieben. \#Der Dozent hat all seinen Kollegen erzählt, sie hat abgeschrieben.

Allerdings handelt es sich bei der zweiten Äußerung um keine satzwertige Proposition, sondern vielmehr um eine Art Rechtsversetzung. Folgt auf den indefiniten Ausdruck ein Verb, ist eine koreferente Interpretation nicht möglich:

(ii) $\quad[\text { The pirate }]_{i}$ shot a monkey on the beach. $[\text { A pirate }]_{* i}$ with a pistol watched everything.

Aus diesem Grund bilden Fälle wie (i) meiner Meinung nach keine Ausnahmen zur CoreferenceRegel.

${ }^{38}$ Dies ist ein Punkt, den Seuren (1991) außer Acht lässt, wenn er (i) als Präsuppositionstest vorschlägt:

(i) Wenn $\mathrm{A} \gg \mathrm{B}$, dann ist $\mathrm{B} \wedge \mathrm{A}$ ein wohlgeformter Ausdruck. 
Im Gegensatz zu nicht-präsupponierten Bedeutungsaspekten zeichnen sich Präsuppositionen demnach durch ein zusätzliches Merkmal aus: Sie müssen immer über eine unterspezifizierte Diskursrelation mit einem Antezedens verknüpft werden und diese Diskursrelation kann dabei den Wert $\mathrm{R}=$ Coreference annehmen. Auch dies stützt die Annahme, dass es sich bei Präsuppositionen um eine Untermenge von [-atissue]-Inferenzen handelt, da sie mit Projektion und ihrer fehlenden Sensibilität für direkte Widerspruchsstrategien alle relevanten Merkmale von [-at-issue]-Inhalten teilen und zusätzlich noch weitere charakteristische Eigenschaften aufweisen.

\subsubsection{Zusammenfassung}

In Kapitel 3 wurde beobachtet, dass alle [-at-issue]-Inferenzen unter Einbettung projizieren und nicht sensibel für direkte Widerspruchsstrategien sind. Somit sind Projektionstests und der sogenannte Hey, wait a minute!-Test nicht zur Ermittlung von Präsuppositionen geeignet. In diesem Kapitel wurde nun gezeigt, dass Präsuppositionen jedoch weitere charakteristische Eigenschaften aufweisen, die nicht von allen [-at-issue]-Inferenzen geteilt werden. So muss der Inhalt einer Präsupposition immer wahr sein, damit die Gesamtäußerung wahrheitskonditional interpretierbar ist, außerdem muss der Inhalt einer Präsupposition immer diskursgebunden sein. Hierbei wurde mit Asher/Lascarides (2003) argumentiert, dass eine Präsupposition immer eine unterspezifizierte Diskursrelation $R$ generiert, über die sie an ein Antezedens angebunden wird. Anders als eindeutig nicht-präsupponierte Inferenzen kann dies ein koreferentes Antezedens sein, in diesem Fall wurde davon ausgegangen, dass die Diskursrelation R den Wert Coreference annimmt. Liegt kein koreferentes Antezedens vor, muss eine Präsupposition über eine andere Relation wie beispielsweise Sub-Part an ein indirektes Antezedens angebunden werden. Da Präsuppositionen mit der Projektion und ihrer fehlenden Sensibilität für direkte Widerspruchsstrategien alle zentralen Eigenschaften von [-at-issue]-Inferenzen aufweisen und sich zusätzlich noch durch zwei weitere Merkmale auszeichnen, kann geschlossen werden, dass Präsuppositionen eine Untermenge der [-at-issue]-Inferenzen bilden, wie in Abbildung 4.1 dargestellt.

\subsection{Welche Nebensätze sind präsupponiert?}

In den Kapiteln 2 und 3 wurden unterschiedliche Nebensätze danach klassifiziert, ob sie dazu geeignet sind, [+at-issue]-Inhalt auszudrücken oder nicht. Eine Frage, die sich daran anschließt, betrifft nun den Status dieser abhängigen Sätze bezogen auf das Konzept der Präsupponiertheit. Die Beantwortung der Frage, welche Nebensätze grundsätzlich präsupponiert sind, ist von entscheidender Bedeutung für die Untersuchung des Auftretens von V2-Stellung in abhängigen Sätzen des Deutschen, die im Zentrum von Teil II der vorliegenden Arbeit steht.

In den meisten Fällen ist die Frage, ob ein abhängiger Satz grundsätzlich präsupponiert ist oder nicht, von der Forschungsliteratur bereits gut untersucht und eindeutig 


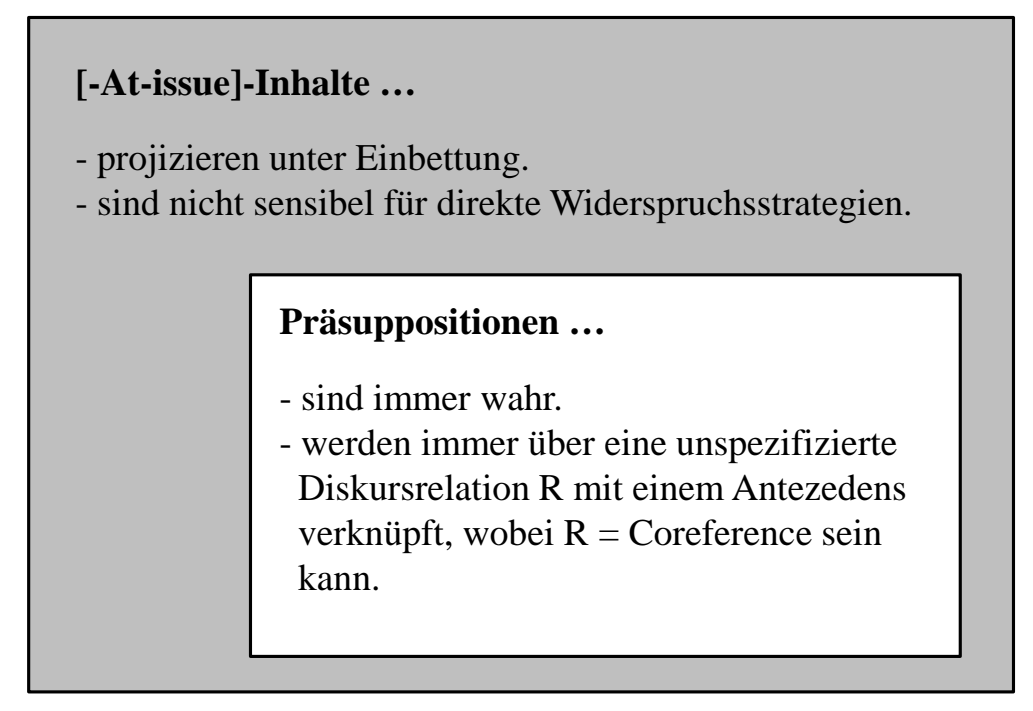

Abbildung 4.1.: Präsuppositionen und [-at-issue]-Inhalte

zu beantworten. Aus diesem Grund werden die entsprechenden Sätze nur kurz diskutiert. Etwas ausführlicher wird dagegen der Status von Temporalsätzen untersucht, da diese in der Forschung zu Präsuppositionen bislang etwas kurz kommen.

Beginnen wir mit der Betrachtung von abhängigen Sätzen, die nicht präsupponiert sind. Aufgrund der Coreference-Regel können wir festhalten, dass appositive Relativsätze grundsätzlich keine präsupponierte Lesart zulassen (vgl. dazu auch Holler 2005, 54 und Roberts et al. 2009 sowie Beispiel (96) in Abschnitt 3.1.1). Als ebenfalls [-präsupponiert] können wir restriktive Relativsätze mit einem indefiniten Antezedens betrachten, da wir bereits oben gesehen haben, dass eine indefinite NP grundsätzlich nicht durch ein koreferentes Antezedens gebunden werden kann. Auch die Komplementsätze von Brückenverben gelten gemeinhin als nicht präsupponiert (vgl. beispielsweise Holler 2008, Hooper/Thompson 1973, Meinunger 2004 und Reis 1997), und auch weil-Sätze sind nach u.a. Antomo/Steinbach (2010), Breindl (2009) und Uhmann (1998) zumindest nicht grundsätzlich präsupponiert. Dasselbe gilt für Komplementsätze von semifaktiven Verben, welche zumindest in bestimmten Kontexten nicht präsupponiert sind (vgl. dazu Hooper/ Thompson 1973, 480 und Karttunen 1971 sowie das Beispiel (43) in Abschnitt 2.2.2). Anders als appositive Relativsätze und restriktive Relativsätze mit einem indefiniten Antezedens lassen weil-Sätze und Komplementsätze von Brücken- und semifaktiven Verben jedoch grundsätzlich eine präsupponierte Lesart zu, da sie, wenn sie keine V2-Stellung aufweisen, via Coreference gebunden werden können.

Keiner der genannten Nebensatztypen, also appositive Relativsätze, restriktive Relativsätze mit einem indefiniten Antezedens, Komplementsätze von Brücken- und semifaktiven Verben und weil-Sätze, weist die für Präsuppositionen typischen Merkmale auf. Wir haben bereits in Kapitel 3 gesehen, dass diese abhängigen Sätze mit Ausnahme von appositiven Relativsätzen nicht projizieren und dass sie sensibel für 
direkte Widerspruchsstrategien sind, womit ihnen schon mal zwei zentrale Eigenschaften von Präsuppositionen fehlen. Außerdem kann die in ihnen ausgedrückte Proposition falsch sein, dennoch ist der Gesamtsatz wahrheitskonditional interpretierbar. So kann Maria beispielsweise die Grünen wählen, dennoch ist man in der Lage, Satz (182-a) einen Wahrheitswert zuzuordnen, und auch die übrigen Sätze in (182) können nach wahr und falsch beurteilt werden, auch wenn die eingebettete Proposition den Wahrheitswert 0 erhalten sollte.

a. Peter glaubt, dass Maria die Piraten wählt.

b. Morgen musst du leider den Bus nehmen, weil unser Auto in der Werkstatt ist.

c. Ich suche ein Buch, das mir eine Freundin empfohlen hat.

d. Die Kanzlerin, die übrigens Physik studiert hat, ist gerade in Afghanistan.

Zudem ist in keinem der Fälle anzunehmen, dass die eingebettete Proposition durch ein direktes oder indirektes Antezedens lizenziert werden muss. Die vier Äußerungen in (182) sind allesamt absolut wohlgeformt, auch wenn die im Nebensatz ausgedrückte Information keinerlei Bezug zu dem vorherigen Diskurs besitzt. Beispielsweise kann Satz (182-c) beim Eintreten in eine Buchhandlung verwendet werden, auch wenn die Nebensatzproposition in diesem Kontext nicht über eine Diskursrelation mit einem Antezedens verknüpft werden kann.

Anders sieht es mit den folgenden Nebensätzen aus. Als [+präsupponiert] werden typischerweise die Komplementsätze von faktiven Verben betrachtet (vgl. beispielsweise Hooper/Thompson 1973, Meinunger 2004 und Reis 1997), dasselbe gilt für restriktive Relativsätze, welche von einem definiten Antezedens abhängen. Für letztere ergibt sich der präsupponierte Status aus der mit der Verwendung eines definiten Artikels einhergehenden Präsupposition, da nominaler Kopf und Relativsatz zusammen eine syntaktische und semantische Konstituente bilden. Dementsprechend weisen die beiden Nebensatztypen auch alle charakteristischen Eigenschaften von Präsuppositionen auf. Dass ihr Inhalt unter Einbettung projiziert und nicht sensibel für einen direkten Widerspruch mit Nein ist, wurde bereits in Kapitel 3 gezeigt. Zusätzlich können wir jetzt noch beobachten, dass die Nebensätze in den folgenden Beispielen wahr sein müssen, damit die Gesamtäußerung wahrheitskonditional interpretierbar ist.

a. Peter ignoriert, dass Maria die Piraten wählt.

b. Ich kenne den Mann, der Marias Fahrrad gestohlen hat.

Auch das vierte relevante Merkmal von Präsuppositionen trifft auf die Sätze zu. So können beide Beispiele nur in solchen Kontexten geäußert werden, in denen der Nebensatzinhalt durch ein koreferentes oder indirektes Antezedens lizenziert wird. Nehmen wir an, dass die Sätze in einem Kontext wie in (184) ohne weiteres Hintergrundwissen verwendet werden, ist die Äußerung aus diesem Grund nicht wohlgeformt. 
a. Was ist los?

b. ?Peter ignoriert, dass Maria die Piraten wählt.

c. ?Ich kenne den Mann, der Marias Fahrrad gestohlen hat.

Während die oben genannten Nebensätze bezüglich ihres präsuppositionalen Status von der Forschung weitgehend einhellig klassifiziert werden, werden temporale Adverbialsätze in diesem Zusammenhang seltener erwähnt. Aus diesem Grund soll im Folgenden kurz diskutiert werden, wie sich Temporalsätze diesbezüglich verhalten. In Kapitel 3 wurde bereits festgestellt, dass Temporalsätze unter Einbettung projizieren und ihrem Inhalt nicht direkt widersprochen werden kann. Bei beiden Merkmalen handelt es sich um notwendige, jedoch nicht um hinreichende Eigenschaften von Präsuppositionen. Wie verhält es sich nun mit den anderen distinktiven Merkmalen von Präsuppositionen? Als Erstes können wir feststellen, dass ein Temporalsatz wahr sein muss, damit dem Gesamtsatz ein Wahrheitswert zugewiesen werden kann. Betrachten wir dazu zuerst den einfachen Satz (185), dessen Wahrheitsbedingungen wir mengentheoretisch angegeben können. Der Satz ist genau dann wahr, wenn das als Daniel bezeichnete Individuum Teil der Menge an Individuen ist, für die gilt, dass sie in s lachen. Andernfalls ist der Satz falsch:

Daniel lacht.

a. $\quad[[\text { Daniel lacht }]]^{s}=1$, wenn Daniel $\in\{\mathrm{x}: \mathrm{x}$ lacht in $\mathrm{s}\}$

b. $\quad[[\text { Daniel lacht }]]^{s}=0$, wenn Daniel $\notin\{\mathrm{x}$ : $\mathrm{x}$ lacht in $\mathrm{s}\}$

Für einen komplexen Satz bestehend aus Haupt- und temporalem Nebensatz wie (186) ist eine solche mengentheoretische Angabe der Wahrheitskonditionen jedoch natürlich nicht ausreichend. Anders als (185) ist der folgende Satz nicht automatisch wahr, wenn Daniel Element der Menge an Individuen ist, für die gilt, dass sie lachen. Stattdessen wird der Hauptsatz bezogen auf einen bestimmten Zeitpunkt evaluiert.

Daniel lacht, als Jenny das Kostüm anprobiert.

Der Wahrheitswert der Hauptsatzproposition hängt von einem temporalen Parameter ab, welcher als Index t der Interpretationsfunktion hinzugefügt wird. Wesentlich ist nun, dass die durch den Temporalsatz ausgedrückte Proposition wahr sein muss, damit die wahrheitsfunktionale Bedeutung des Gesamtsatzes bestimmt werden kann. Ist, bezogen auf unser Beispiel (186), die Proposition Jenny probiert das Kostüm an falsch, kann nicht darüber entschieden werden, ob der Gesamtsatz wahr ist oder nicht, da nicht klar ist, bezogen auf welchen Zeitpunkt er evaluiert werden muss. Dies bedeutet, dass sich Temporalsätze genauso verhalten wie beispielsweise faktive Komplementsätze, die ebenfalls wahr sein müssen, damit dem Gesamtsatz ein Wahrheitswert zugewiesen werden kann. Demnach können aus dem Bedeutungstupel (143) auch für Temporalsätze nur die ersten beiden Fälle wahrheitskonditional berechnet werden. ${ }^{39}$

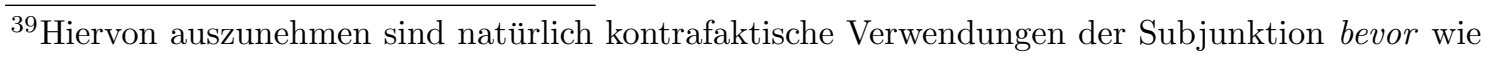
in dem folgenden Beispiel, die ja gerade ausdrücken, dass die entsprechende Proposition nicht 
Wie sieht es nun mit der zweiten, für Präsuppositionen als distinktiv erachteten Eigenschaft, nämlich der Beobachtung, dass Präsuppositionen immer über eine unpezifizierte Diskursrelation $\mathrm{R}$ angebunden sein müssen, aus? Beginnen wir dazu gleich mit der Tatsache, dass Temporalsätze selbstverständlich informativ sein können, was der folgende Textausschnitt exemplarisch belegt, in dem der fettgedruckte Adverbialsatz eindeutig eine neue Information einführt. ${ }^{40}$

(187) Von 1889 bis 1893 besuchte Kafka die Deutsche Knabenschule am Fleischmarkt in Prag. Dann wechselte er, entsprechend der Auswahl seines Vaters, an das humanistische Staatsgymnasium in der Prager Altstadt, welches sich im selben Gebäude wie das Galanteriegeschäft seines Vaters befand. Auch an dieser Schule war die Unterrichtssprache Deutsch. (...) Nachdem er sein Abitur im Jahre 1901 mit ,befriedigend“ bestanden hatte, verließ er zum ersten Mal in seinem Leben Böhmen und reiste mit seinem Onkel Siegfried Löwy nach Norderney und Helgoland.

Derartige Beispiele sprechen jedoch nicht grundsätzlich gegen eine Analyse von Temporalsätzen als präsupponierte Ausdrücke, da auch andere von der Literatur einhellig als Präsuppositionen bezeichnete Ausdrücke informativ sein können, wie wir in Abschnitt 4.1.2 bereits gesehen haben. Es spricht sogar einiges dafür, dass informative Präsuppositionen sehr häufig oder sogar häufiger auftreten als Präsuppositionen, die eine bekannte Information ausdrücken, wie Schwarz (1998) bezogen auf definite NPn gezeigt hat. Die Verwendung eines informativen Temporalsatzes wie in Beispiel (187) ist dann so zu erklären, dass der im Adverbialsatz ausgedrückte Inhalt akkommodiert werden muss, wobei Akkommodation nach Asher/Lascarides (1998b) und Asher/Lascarides (2003) als Diskursanbindung über eine rhetorische Relation $\mathrm{R}$ zu verstehen ist. In (187) ist es aufgrund der spezifischen Textsorte Biografie beispielsweise erwartbar, dass eine Lebensstation nach der anderen genannt wird.

wahr ist.

(i) Bevor Peter die Gäste beleidigen konnte, wurde er rausgeschmissen.

Lakoff (1970) schlägt bezogen auf kontrafaktische Verwendungen des englischen before vor, dass die Subjunktion je nach Kontext zwei verschiedene Arten von Präsuppositionen auslösen kann - eine faktische oder aber eine kontrafaktische, es gilt also $q$ before $p \gg$ p oder $q$ before $p \gg \neg$ p. Heinämäki (1972) entwickelt dies weiter und beschäftigt sich mit der Frage, welche Bedingungen darüber entscheiden, welche der beiden möglichen Präsuppositionen jeweils ausgelöst wird. Die Frage, ob es sich bei kontrafaktischen Inferenzen tatsächlich um Präsuppositionen handelt, muss an dieser Stelle unbeantwortet bleiben. Sicher ist aber Eines: Das in einem kontrafaktischen Temporalsatz ausgedrückte Ereignis muss zumindest als Möglichkeit im Kontext gegeben sein, da die Äußerung andernfalls pragmatisch nicht wohlgeformt wäre. So ist es aus pragmatischer Sicht nicht relevant, eine Aussage über das Nicht-Eintreffen eines Ereignisses zu machen, wenn sein Eintreffen nie zur Debatte stand. Geht man nun von einem anaphorischen Präsuppositionsbegriff aus, so kann dies durchaus als Bindung des durch den kontrafaktischen Temporalsatz eingeführten Diskursreferenten durch ein Antezedens interpretiert werden.

${ }^{40}$ Das Beispiel stammt von der Website http://de.wikipedia.org/wiki/Franz_Kafka, letzter Zugriff am 02.03.2011. 


\section{Präsupponiertheit und At-issueness}

Genauer gesagt ist anzunehmen, dass das im Temporalsatz ausgedrückte Ereignis, also das Bestehen des Abiturs, über die Relation $\mathrm{R}=$ Elaboration an den vorher erwähnten Schulbesuch angeknüpft wird (eine exakte Modellierung der diskurssemantischen Funktion von Temporalsätzen erfolgt in Teil III). Ein anderes Beispiel für eine solche Bridging-Relation zwischen einem informativen Temporalsatz und dem Diskurskontext bildet (188). Hier ist anzunehmen, dass der durch den Temporalsatz eingeführte Referent $\pi_{2}$ über die Relation $\mathrm{R}=$ Sub-Part an den Referenten $\pi_{1}$ (= Jan ging in den Supermarkt) angeknüpft wird, da sich $\pi_{2}$ auf ein Teilereignis des größeren Ereignisses Supermarktbesuch bezieht.

(188) Nach der Arbeit ging Jan in den Supermarkt. Nachdem er drei Liter Cola und verschiedene Sorten Tiefkühlpizza in seinem Einkaufswagen verstaut hatte, versuchte er auszumachen, welche Kasse die schnellste war.

Wenn Temporalsätze tatsächlich präsupponiert sind, sollten sich nun auch Kontexte finden lassen, in denen die Diskursanbindung eines informativen Temporalsatzes nicht möglich ist. Lascarides/Oberlander $(1993,261)$ liefern hierfür folgendes Beispiel:

(189) I'm not a useless driver.

a. I coud drive before you were born.

b. ?You were born after I could drive.

Obwohl die Äußerungen (189-a) und (189-b) synonym sind, was den Ausdruck der temporalen Relation betrifft, ist nur (189-a) in diesem Kontext wohlgeformt. Lascarides/Oberlander (1993) erklären die Markiertheit von (189-b) nun damit, dass der Inhalt des Temporalsatzes nicht akkommodiert werden kann, da keine Diskursrelation zu einem geeigneten Antezedens hergestellt werden kann. Auch die Markiertheit des folgenden Beispiels kann darauf zurückgeführt werden, dass der im Temporalsatz ausgedrückte Inhalt nicht an den Diskurs angebunden werden kann. In dem kurzen Dialog in (190) klingt B's Antwort auf die Frage von A zumindest markiert. ${ }^{41}$

A: Was machst du eigentlich in den Sommerferien?

B: ?Bevor ich Verwandte in Hongkong besuche, fliege ich nach Indonesien.

Würde die temporale Subjunktion bevor lediglich dem Ausdruck eines relationalen zeitlichen Verhältnisses zwischen zwei Propositionen dienen, wäre die Markiertheit des Beispiels unerwartet. Stattdessen bildet B in diesem Kontext nur dann eine wohlgeformte Antwort, wenn A bereits weiß, dass B Verwandte in Hongkong besuchen

${ }^{41}$ Der Satz wird besser, wenn das Adverb dann eingefügt wird und der Temporalsatz im Nachfeld steht:

Ich fliege nach Indonesien, bevor ich dann Verwandte in Hongkong besuche.

Geurts (1999) bezeichnet solche Ausdrücke, die die Akkommodation einer Präsupposition erleichtern, als Präsuppositionsbrücken (presuppositional bridges). 
wird. Dies kann darauf zurückgeführt werden, dass der Adverbialsatz eine präzise temporale Einordnung des Hauptsatzes ermöglichen soll. Genau wie die PP von Ende Juli bis Mitte August in Beispiel (191) dazu dient, den Hauptsatz auf einem Zeitstrahl zu verorten, erfüllt auch ein temporaler Adverbialsatz dieselbe Funktion.

Ich fliege von Ende Juli bis Mitte August nach Indonesien.

Wie die PP in (191) dient auch der Adverbialsatz in (190) als zeitlicher Anker für den Hauptsatz (eine genaue semantische Beschreibung von Temporalsätzen steht im Zentrum von Teil III). Diese Funktion kann er aber nur dann erfüllen, wenn die in ihm ausgedrückte Information bekannt ist oder über eine Diskursrelation und ein indirektes Antezedens lizenziert wird. Da A's Frage in Beispiel (190) aber nahelegt, dass A keinerlei Informationen über B's Sommerplanung besitzt, ist die Diskursanbindung des Temporalsatzes in diesem Fall nicht möglich.

Wir können festhalten, dass Temporalsätze immer wahr sein müssen, damit die Gesamtäußerung wahrheitskonditional interpretierbar ist, zudem drücken sie eine Information aus, die immer über ein koreferentes oder ein indirektes Antezedens lizenziert werden muss. Letzteres konnte durch die Existenz von markierten Fällen wie (189-b) und (190) belegt werden, in denen die im Temporalsatz ausgedrückte Information nicht akkommodiert werden kann. Im Folgenden werde ich deshalb genau wie u.a. Geurts (1999), Hara (2008), Heinämäki (1972), Johnston (1994), Kadmon (2001), Kamp/Reyle (1993), Karttunen (1973), Lakoff (1970), Lascarides/Oberlander (1993), Sawada/Larson (2004), Schilder/Tenbrink (2001), Tenbrink (2007) und Van der Sandt $(1992,342)$ davon ausgehen, dass der Inhalt von Temporalsätzen genau wie der von beispielsweise faktiven Komplementsätzen präsupponiert ist. Drückt ein Temporalsatz eine diskursneue Information aus, muss er über eine Diskursrelation an ein Antezedens angebunden werden, was ich im Folgenden als temporales Bridging bezeichnen werde. Kann keine solche Relation gefunden werden, ist die Äußerung nicht wohlgeformt (für mehr Details vgl. Teil III).

Die Diskussion hat gezeigt, dass die abhängigen Sätze, welche auch in der Lage sind, [+at-issue]-Inhalt auszudrücken, nicht grundsätzlich präsupponiert sind, auch wenn sie eine präsupponierte Lesart zulassen. Von den vier Nebensatztypen aus Gruppe 2 dagegen, welche prinzipiell immer [-at-issue]-Inhalt enthalten, sind drei grundsätzlich präsupponiert. Appositive Relativsätze dagegen können keine präsupponierten Bedeutungsaspekte enthalten, obwohl sie ebenfalls nicht dazu geeignet sind, ohne ihren Bezugssatz [+at-issue]-Inhalt auszudrücken. Diese Ergebnisse, die noch einmal in Tabelle 4.1 zusammengefasst sind, decken sich mit der weiter oben getroffenen Aussage, dass Präsuppositionen eine Untermenge der [-at-issue]-Inhalte bilden.

\subsection{Zusammenfassung des Kapitels}

In diesem Kapitel wurde anhand typischer Eigenschaften von Präsuppositionen untersucht, in welchem Verhältnis Präsupponiertheit und At-issueness zueinander stehen. Wie [-at-issue]-Inferenzen projizieren Präsuppositionen und sind nicht sensibel 


\begin{tabular}{|l||c|c|}
\hline Nebensatztyp & [at-issue] & [präsupponiert] \\
\hline \hline Komplementsatz, Brückenverb & $+/-$ & $+/-$ \\
\hline Semifaktiver Komplementsatz & $+/-$ & $+/-$ \\
\hline Faktiver Komplementsatz & - & + \\
\hline Temporalsatz & - & + \\
\hline Adverbialsatz mit weil & $+/-$ & $+/-$ \\
\hline Appositiver Relativsatz & - & - \\
\hline Restriktiver Relativsatz, definites Antezedens & - & + \\
\hline Restriktiver Relativsatz, indefinites Antezedens & $+/-$ & $+/-$ \\
\hline
\end{tabular}

Tabelle 4.1.: Klassifikation der Nebensätze nach At-issueness und Präsupponiertheit

für einen direkten Widerspruch mit Nein. Zusätzlich wurde gezeigt, dass sie zwei weitere Merkmale aufweisen, in denen sie sich von [-at-issue]-Inhalten unterscheiden: Sie müssen immer wahr sein und sie müssen durch ein direktes oder indirektes Antezedens im Diskurskontext lizenziert sein. Es wurde bezogen auf den zweiten Punkt argumentiert, dass Präsuppositionen anders als eindeutig nicht-präsupponierte Inferenzen über die Diskursrelation $\mathrm{R}=$ Coreference mit einem direkten Antezedens oder aber über eine andere Relation wie beispielsweise $\mathrm{R}=$ Sub-Part an ein indirektes Antezedens geknüpft werden können.

Präsuppositionen weisen mit der Projektion und ihrer fehlenden Sensibilität gegenüber direkten Widerspruchsstrategien die zwei relevanten Merkmale von [-atissue]-Inhalten auf und können zusätzlich noch durch zwei weitere distinktive Eigenschaften charakterisiert werden. Daraus folgt, dass Präsuppositionen eine Untermenge der [-at-issue]-Inhalte bilden. Hierzu passt auch die in Abschnitt 4.2 vorgenommene Klassifikation der in dieser Arbeit untersuchten acht abhängigen Sätze: Während die [+/-at-issue]-Nebensätze aus Gruppe 1 alle [-präsupponiert] sind, grundsätzlich aber auch eine präsupponierte Lesart zulassen, sind die [-at-issue]Sätze aus Gruppe 2 mit Ausnahme der appositiven Relativsätze alle [+präsupponiert].

Dass Präsuppositionen immer [-at-issue] sind, ist dabei nicht überraschend - schließlich werden Präsuppositionen traditionell als Hintergrundannahmen definiert. 
Teil II.

Abhängige V2-Strukturen 



\section{Ein erster Überblick: Wurzelphänomene in abhängigen Sätzen}

In Kapitel 2.2 wurde gezeigt, dass bestimmte Nebensatztypen, die als Gruppe 2 zusammengefasst wurden, je nach Kontext entweder zur Haupt- oder zur Nebenstruktur gehören können. Ihre diskursstrukturelle Funktion ist demnach kontextabhängig. Beispielsweise ist der eingebettete Satz in dem folgenden Beispiel ambig, da er je nach Kontext entweder [+at-issue]- oder [-at-issue]-Inhalt ausdrücken kann. Wird der Satz zur Beantwortung der Frage $\mathrm{Q}=$ Wer hat den Kuchen gegessen? geäußert, gehört der Nebensatz eindeutig zur Hauptstruktur. Geht es im Kontext dagegen um die Frage, wer alles glaubt, dass Jenny den Kuchen gegessen hat, drückt der eingebettete Satz [-at-issue]-Inhalt aus.

(192) Evi glaubt, dass Jenny den Kuchen gegessen hat.

Daraus ergibt sich nun aber die Frage, auf welche Weise diese Ambiguität aufgelöst wird, wenn die aktuelle $\mathrm{Q}$ nicht explizit im Kontext gegeben ist. Bereits in Abschnitt 2.1.3 wurde gezeigt, dass die diskursstrukturelle Funktion einer Äußerung oder eines Äußerungsteils sprachlich markiert sein kann. Als ein Beispiel für eine konventionelle Markierung der Hauptstruktur wurde in Abschnitt 2.1.3 die Fokusintonation genannt, da der Träger eines Fokusmerkmals nicht Teil der Nebenstruktur sein kann (vgl. Beispiel (32) sowie Onea 2011). Gleichzeitig haben wir in Abschnitt 2.1.3 jedoch gesehen, dass derartige sprachliche Markierungen die mögliche Q zwar einschränken, jedoch nicht eindeutig festlegen.

In den folgenden Abschnitten soll für die These argumentiert werden, dass das Deutsche über ein ganz spezifisches syntaktisches Mittel verfügt, um anzuzeigen, ob ein abhängiger Satz Teil der Haupt- oder der Nebenstruktur ist. Genauer gesagt handelt es sich dabei um das gut bekannte Phänomen, dass abhängige Sätze im Deutschen in bestimmten Kontexten die sonst auf selbstständige Sätze beschränkte V2-Stellung aufweisen können. Beispielsweise kann der Komplementsatz in (193) alternativ zu der kanonischen VL-Stellung wie in (193-a) auch mit V2-Stellung wie in (193-b) gebildet werden.

(193) a. Mailin glaubt, dass Daniel den Kuchen aufgegessen hat.

b. Mailin glaubt, Daniel hat den Kuchen aufgegessen. 
Auch andere abhängige Sätze wie weil-Sätze können zumindest in der gesprochenen Sprache V2-Stellung aufweisen, andere Nebensätze dagegen blockieren dies grundsätzlich (ein Überblick über die wichtigsten Lizenzierungskontexte wird in Abschnitt 6 geliefert). Das Ziel der folgenden Abschnitte besteht nun darin, zu zeigen, dass es sich bei V2-Stellung in abhängigen Sätzen um eine optionale Markierung für At-issueness handelt.

V2-Stellung in abhängigen Sätzen kann unter dem Begriff der Wurzelphänomene oder auch Main Clause Phenomena subsumiert werden. Dabei handelt es sich um zumeist syntaktische Transformationen, deren Auftreten typischerweise auf selbstständige Sätze beschränkt ist, die in bestimmten Kontexten aber auch in abhängigen Sätzen auftreten können. In der englischsprachigen Literatur werden derartige Phänomene auch unter dem Begriff der Embedded Root Phenomena zusammengefasst - da es sich bei den betroffenen Sätzen jedoch nicht ausschließlich um integrierte, sondern auch um unintegrierte abhängige Sätze handelt (vgl. dazu Abhschnitt 6.2) wird auf die Verwendung dieses Begriffs im Folgenden verzichtet.

Die Erfoschung von Wurzelphänomenen geht zurück auf die Dissertation von Emonds (1970), in der zwischen zwei Arten von syntaktischen Transformationen unterschieden wird. Bei einer strukturkonservierenden Transformation (structure-preserving transformation) wird ein Knoten in eine Zielposition bewegt, in der auch ein Knoten derselben Kategorie basisgeneriert werden kann. Ein Beispiel hierfür bildet das Passiv, in dem typischerweise die Subjekt-NP des Passivsatzes in die Spezifikatorposition des C-Systems verschoben wird, also in eine Position, in der der allgemeinen Phrasenstrukturregel $\mathrm{S} \rightarrow \mathrm{NP}+\mathrm{VP}$ zufolge sowieso eine NP steht (für mehr Details vgl. Emonds 1970, 34ff). Strukturerhaltende Transformationen sind in allen abhängigen und selbstständigen Sätzen möglich, wie die folgenden zwei Beispielsätze belegen.

a. Der Kuchen wurde von Daniel aufgegessen.

b. Jenny bedauert, dass der Kuchen von Daniel aufgegessen wurde.

Demgegenüber stehen die Wurzeltransformationen, bei denen eine Konstituente in den höchsten Knoten, wo sie direkt von S dominiert wird, bewegt wird. Emonds (1970) liefert hierfür zahlreiche Beispiele aus dem Englischen, u.a. Subjekt-AuxiliarInversion, Topikalisierung und Linksversetzungen, aber auch die V2-Bewegung im Deutschen (vgl. hierzu Emonds 1970, 10f). Während strukturerhaltende Transformationen in selbstständigen und abhängigen Sätzen auftreten können, wie die Sätze in (194) belegen, beobachtet Emonds (1970) bezogen auf Wurzeltransformationen, dass diese nie in abhängigen Sätzen möglich sind. In den folgenden Beispielen aus Emonds $(1970,24)$ (dort die Beispiele 42 und 43) führt die Topikalisierung des Objekts nur im selbstständigen Satz zu einer grammatischen Struktur.

(195) a. These steps I used to sweep with a broom.

b. *Have I shown you the broom (that) these steps I used to sweep with? 
Dasselbe gilt für die V2-Bewegung im Deutschen, deren Auftreten typischerweise auf selbstständige Sätze wie (196-a) beschränkt ist und in einem Nebensatz wie (196-b) zu einer ungrammatischen Struktur führt.

a. Peter ist gerade in Mainz.

b. *Maria ignoriert, Peter ist gerade in Mainz.

Die Auffassung, dass Wurzelphänomene nur in selbstständigen Sätzen auftreten können, wird allerdings drei Jahre später durch Hooper/Thompson (1973) widerlegt, die beobachten, dass diese Distribution nur partiell korrekt ist. So kann eine Untergruppe an Nebensätzen durchaus Wurzeltransformationen aufweisen, wie die Beispiele (197) und (193-b) belegen. In (197) ist der Nebensatz trotz Topikalisierung des direkten Objekts wohlgeformt, dasselbe gilt für den V2-Nebensatz in (193-b), der sich von der ungrammatischen Struktur in (196-b) nur im Matrixprädikat unterscheidet.

John believes that this book, Mary read.

Hooper/Thompson (1973) nennen noch zahlreiche weitere, zumeist strukturelle Transformationen wie die Voranstellung der Negation, VP-Voranstellung oder Linksversetzungen, die alle auch in spezifischen abhängigen Kontexten auftreten können.

Ein Komplementsatz mit V2-Stellung wie in Beispiel (193-b) bildet eine hybride Struktur, die sich Eigenschaften mit Haupt- und Nebensätzen teilt (die Eigenschaften dieser nicht-kanonischen Sätze werden ausführlicher in u.a. Frank 2000, Helbig/Kempter 1974, Holler 2008, Meinunger 2005, Reis 1997 und Truckenbrodt 2006 diskutiert). Zum einen ist der vom Matrixprädikat selegierte Satz aufgrund seines Komplementstatus eindeutig abhängig, zum anderen fehlt aber der nebensatzeinleitende Komplementierer, wodurch die sonst auf Hauptsätze beschränkte V2-Bewegung erst möglich wird.

In V2-Sätzen wird nach der Analyse von den Besten (1983) das finite Verb nach $\mathrm{C}^{0}$ und eine XP in die Spezifikatorposition von $\mathrm{C}$ bewegt. Daraus folgt, dass V2Stellung nur in Sätzen, in denen $\mathrm{C}^{0}$ nicht durch einen Komplementierer besetzt wird, möglich ist. Bezogen auf das Auftreten des finiten Verbs und eines Komplementierers in der Kopfposition von $\mathrm{C}$ besteht demnach komplementäre Distribution. Aus diesem Grund ist das folgende Beispiel ungrammatisch:

*Maria glaubt, dass Peter ist gerade in Mainz.

Dagegen können abhängige Sätze in anderen germanischen Sprachen wie dem Friesischen, dem Dänischen oder dem Norwegischen auch dann V2-Stellung aufweisen, wenn der Nebensatz durch einen overten Komplementierer eingeleitet wird, wie das folgende Beispiel aus dem Friesischen illustriert (vgl. zu abhängigen V2-Strukuren im Skandinavischen Andersson 1975, Bentzen et al. 2007, Haan/Weerman 1985, Heycock 2005, Iatridou/Kroch 1992 und Vikner 1995). 
Ik leau dat hy kin him wol rêde.

Ich glaube dass er kann sich selbst sicherlich retten

'Ich glaube, dass er auf sich aufpassen kann.'

Sätze wie (199) widerlegen erst einmal die Voraussage von den Besten (1983). Um diesen Widerspruch zu lösen, wurden derartige Sätze, in denen V2-Stellung und ein overter Komplementierer gleichzeitig auftreten, als Fälle von CP-Rekursion analysiert. Dies bedeutet, dass der Komplementierer die Kopfposition einer höheren CP einnimmt, welche eine weitere CP als ihr Komplement selegiert, in deren Kopf wiederum das finite Verb steht (vgl. dazu u.a. Rizzi/Roberts 1996 und Vikner 1995). Das Friesische und die oben genannten skandinavischen Sprachen gehören zu den sogenannten Limited Embedded V2-Sprachen, da das Auftreten von V2-Stellung in eingebetteten Sätzen nur in bestimmten Lizenzierungskontexten möglich ist. Während das Verb leauwe ('glauben') in Beispiel (199) einen V2-Satz einbetten kann, ist diese Möglichkeit nicht für das faktive Verb bedauern möglich, weswegen der folgende Beispielsatz nach Iatridou/Kroch $(1992,4)$ ungrammatisch ist.

(200) *Pyt betreuret dat hy hie my sjoen.

Pyt bedauert dass er hat mich gesehen

Wir haben bereits gesehen, dass das Auftreten eines V2-Nebensatzes auch im Deutschen nicht in jedem Kontext wohlgeformt ist (vgl. Beispiel (196-b) sowie den Überblick über die wichtigsten Lizenzierungskontexte in Abschnitt 6). Aus diesem Grund wird das Deutsche zumeist ebenfalls zu der Gruppe der Limited Embedded V2Sprachen gezählt, wobei sich das Deutsche von den anderen Sprachen dieser Gruppe wohlgemerkt darin unterscheidet, dass V2-Stellung nur unter Wegfall des Komplementierers möglich ist, Deutsch ist demnach eine sogenannte Comp-drop-Sprache. Im Unterschied zu den Limited Embedded V2-Sprachen ist das Auftreten von V2Nebensätzen im Isländischen und im Jiddischen, den General Embedded V2-Sprachen, nicht auf bestimmte Lizenzierungskontexte beschränkt. Auch in diesen Sprachen liegt V2-Stellung bei gleichzeitiger Einleitung durch einen Komplementierer vor, weswegen diese Sätze ebenfalls als CP-Rekursion analysiert werden (vgl. beispielsweise Haan/Weerman 1985). Für die Erforschung von Wurzelphänomenen sind die General Embedded V2-Sprachen zumeist von geringerem Interesse als die Limited Embedded V2-Sprachen, da die Verbbewegung keinen speziellen strukturellen oder funktionalen Lizenzierungsbedingungen unterliegt.

Die Beobachtung, dass Wurzeltransformationen auch in abhängigen Sätzen möglich sind, markiert den Beginn einer langen Forschungstradition (vgl. beispielsweise Antomo 2012a, Antomo/Steinbach 2010, Axel-Tober 2012, Bentzen et al. 2007, den Besten 1983, Bianchi/Frascarelli 2009, Brandner 2004, Breindl 2009, Coniglio 2007, Ebert et al. 2006, Frank 2000, Frey 2011, Gärtner 2001, Green 1976, Haan/Weerman 1985, Haegeman 2003, Haegeman 2007, Heycock 2005, Heycock/Sorace 2007, Holler 2008, Iatridou/Kroch 1992, Julien 2007, Kiparsky/Kiparsky 1970, Küper 1991, Maki et al. 1999, Meinunger 2004, Pasch 1997, Pittner 1999, Reis 1995, Reis 1997, Rinas 1997, Sawada/Larson 2004, Scheffler 2005, Scheutz 1998, Simons 2007, Stein- 
bach 2007, Truckenbrodt 2006, Uhmann 1998, Vikner 1995, Volodina 2011, Wechsler 1991, Weerman 1989, Wegener 1993, Wegener 2000 und Wiklund et al. 2009). Die Tatsache, dass bestimmte Nebensätze hauptsatzähnliche Eigenschaften aufweisen, indem sie sonst auf Wurzelkontexte beschränkte syntaktische Transformationen untergehen können, ist dabei ein sprachübergreifendes, universelles Phänomen.

Die am besten untersuchten Hauptsatzphänomene sind typischerweise syntaktische Transformationen, wie sie bereits Emonds (1970) beschrieben hat. Strukturelle Hauptsatzphänomene involvieren klassischerweise die Bewegung eines Elements in die linke Satzperipherie, wie sie beispielsweise bei Topikalisierungen, der V2-Bewegung oder Subjekt-Auxiliar-Inversion vorliegt. Eine selektive Übersicht über einige der strukturellen Hauptsatzphänomene, zumeist Voranstellungstransformationen, wie sie in der Literatur notiert wurden, findet sich in Heycock (2005). Neben derartigen syntaktischen Transformationen gibt es aber auch gute Gründe, das Auftreten einiger spezifischer lexikalischer Elemente unter dem Begriff der Wurzelphänomene zu subsumieren. Wie in u.a. Coniglio (2007), Frey (2011), Green (1976), Haegeman (2002) und Reis (1997) argumentiert, stellt auch die Möglichkeit, Modalpartikeln oder adverbiale Adjunkte, die die Sprechereinstellung ausdrücken, zu enthalten, eine haupsatzähnliche Eigenschaft dar. So kann der Komplementsatz in Beispiel (201-a) (nach Meibauer 1994, 135) die Modalpartikel ja enthalten, dieselbe Möglichkeit ist dagegen nicht für das Komplement des Verbs ignorieren gegeben.

(201) a. Mir ist eingefallen, dass Natassja ja verheiratet ist.

b. \#Peter ignoriert, dass Natassja ja verheiratet ist.

In ihrem Auftreten sind Wurzelphänomene auf eine Untergruppe an abhängigen Sätzen beschränkt. Während beispielsweise ein Verb wie glauben einen V2-Satz einbetten kann, selegiert das Verb ignorieren immer einen VL-Satz als Komplement. In der Definition der Gruppe an Nebensätzen, welche Wurzelphänomene zulassen, liegt ein wesentliches Ziel der Erforschung von Wurzelphänomenen. Eine interessante Frage ist dabei, ob es sich sprach- und phänomenübergreifend um dieselben Lizenzierungskontexte handelt und wie diese Kontexte beschaffen sind. Ein kurzer Überblick über die wichtigsten abhängigen Sätze, in denen im Deutschen V2-Stellung auftreten kann, findet sich in Abschnitt 6, im Vergleich dazu wird das Auftreten von Voranstellungstransformationen im Englischen in Abschnitt 7.3.2 untersucht.

Neben der Definition der Lizenzierungskontexte besteht ein zweites zentrales Ziel der Arbeiten zu Wurzelphänomenen darin, die ihrem Auftreten zugrundeliegenden Prinzipien zu definieren. Von der Grammatiktheorie zu klären ist dabei vor allem, weshalb das Auftreten spezifischer syntaktischer und lexikalischer Phänomene auf spezielle Lizenzierungskontexte beschränkt ist und welche relevanten strukturellen oder funktionalen Eigenschaften ein solcher Kontext besitzen muss, um Wurzelphänomene zu lizenzieren. Im Wesentlichen können hier zwei grundlegend unterschiedliche Herangehensweisen unterschieden werden: Strukturelle Ansätze erklären das Auftreten von hauptsatztypischen Transformationen in abhängigen Kontexten primär mithilfe von syntaktischen Annahmen wie beispielsweise Beschränkungen von Be- 
wegung. Funktionale Ansätze dagegen gehen davon aus, dass nicht-strukturelle, also semantische und pragmatische Faktoren für das Auftreten von Wurzelphänomenen verantwortlich sind. Der wichtigste funktionale Ansatz geht dabei auf die Untersuchungen von Hooper/Thompson (1973) zum Englischen zurück und beruht auf der Beobachtung, dass nur assertive Nebensätze Wurzelphänomene aufweisen können. Diese sogenannte Assertionshypothese und deren Übertragung auf die Distribution von eingebetteter V2-Stellung im Deutschen wird in Abschnitt 7.1 diskutiert. Wir werden sehen, dass das Auftreten von V2-Stellung in abhängigen Sätzen mithilfe dieses Ansatzes nur bedingt erklärbar ist. Aus diesem Grund wird in Abschnitt 7.2 eine alternative Herangehensweise, die sogenannte At-issueness-Hypothese, vorgestellt und es wird dafür argumentiert, dass V2-Stellung nur in [+at-issue]-Kontexten auftreten kann. Anschließend werden in Abschnitt 7.3 weitere Anwendungsbereiche der At-issueness-Hypothese diskutiert. Zuvor werden im folgenden Kapitel jedoch erst einmal die acht Nebensätze aus den Gruppen 1 und 2 daraufhin untersucht, ob sie V2-Stellung aufweisen können. 


\section{Lizenzierungskontexte}

Wie bereits im vorherigen Abschnitt erwähnt, besteht ein wesentliches Ziel der Forschungen zu Wurzelphänomenen darin, die Untergruppe an Nebensätzen zu definieren, welche Wurzelphänomene lizenzieren. Deswegen werden im Folgenden die acht Nebensatztypen aus den Gruppen 1 und 2 daraufhin untersucht, ob sie im Deutschen V2-Stellung aufweisen können. ${ }^{42}$

\subsection{Komplementsätze}

Beginnen wir mit Komplementsätzen. Wie in Abschnitt 2.2 bei der Untersuchung der Diskursfunktion von Komplementsätzen spielt auch bezogen auf das Auftreten von V2-Stellung das Matrixprädikat eine entscheidende Rolle. Es ist altbekannt, dass die sententialen Komplemente von Sagens- und Einstellungsprädikaten wie in den folgenden Beispielen entweder VL- oder unter Wegfall des Komplementierers V2-Stellung aufweisen können (vgl. für mehr Details u.a. Auer 1998, Frank 2000, Freywald 2013, Holler 2008, Meinunger 2004, Reis 1997 und Truckenbrodt 2006, sowie Andersson 1975, Brandner 2004, Haan/Weerman 1985, Heycock/Sorace 2007, Iatridou/Kroch 1992, Julien 2007, Vikner 1995, Wechsler 1991 und Weerman 1989 zu V2-Komplementsätzen in anderen germanischen Sprachen und Axel-Tober 2012 zur historischen Entwicklung von V2-Komplementsätzen).

(202) a. Jenny hat gesagt, dass Daniel die Donuts gegessen hat.

b. Jenny hat gesagt, Daniel hat die Donuts gegessen.

(203) a. Jenny glaubt, dass Daniel die Donuts gegessen hat.

b. Jenny glaubt, Daniel hat die Donuts gegessen.

Auch Nebensätze, die von einem semifaktiven Prädikat selegiert sind, können V2Stellung aufweisen, wie Meinunger (2004) beobachtet.

(204) Ich habe gerade erfahren, ich bin durchgefallen.

\footnotetext{
${ }^{42}$ Natürlich handelt es sich bei dem Auftreten von V2-Stellung in abhängigen Kontexten um ein größeres Phänomen, das mehr als die im Folgenden untersuchten acht Nebensatztypen betrifft und zu dem bereits zahlreiche Arbeiten existieren. Somit handelt es sich bei den folgenden Abschnitten nur um eine auszugsweise Diskussion des Phänomens, wobei bei der Wahl der acht Nebensatztypen jedoch darauf geachtet wurde, mit Attribut-, Komplement- und Adverbialsätzen die drei zentralen Nebensatzfunktionen abzudecken. Einige weitere Nebensätze werden bezogen auf das Auftreten von V2-Stellung in Abschnitt 7.3.1 diskutiert.
} 


\section{Lizenzierungskontexte}

Im Gegensatz zu semifaktiven und Brückenverben lizenzieren unter faktiven Verben eingebettete Komplementsätze typischerweise keine V2-Stellung (vgl. auch Beispiel (196-b) weiter oben). Aus diesem Grund sind die beiden folgenden Sätze ungrammatisch, in denen ein Berücksichtigungs- und ein emotives Prädikat jeweils einen V2-Satz einbetten.

a. *Jenny ignoriert, Daniel hat die Donuts gegessen.

b. *Jenny nimmt Daniel übel, er hat die Donuts gegessen.

Wir können festhalten, dass nur ein Teil der Komplementsätze V2-Stellung aufweisen kann. Entscheidend ist dabei das Matrixprädikat. Während Brücken- und semifaktive Verben auch einen V2-Satz selegieren können, ist V2-Stellung in den Komplementen von faktiven Verben ausgeschlossen. ${ }^{43}$

\subsection{Adverbialsätze}

Bei der Betrachtung von Adverbialsätzen beschränke ich mich wie auch schon in den vorherigen Kapitel auf Temporalsätze und mit weil eingeleitete Sätze. Dass temporale Adverbialsätze Wurzelphänomene blockieren, wurde bereits von zahlreichen Autoren für unterschiedliche Sprachen festgestellt (vgl. vor allem Sawada/Larson 2004, aber auch Andersson 1975 und Haegeman 2007). Bezogen auf das Deutsche beobachten Antomo (2012b), Altmann (1997), Blühdorn (2004b), Breindl (2009) und Pasch et al. (2003, 440), dass Temporalsätze nie V2-Stellung aufweisen können. Aus diesem Grund ist der folgende Satz ungrammatisch.

(206) *Doris kam in die Küche, als Daniel verspeiste gerade den letzten Bissen vom Kuchen.

Dagegen ist es ein bekanntes und gut untersuchtes Phänomen, dass ein mit weil eingeführter Adverbialsatz im gesprochenen Deutsch auch V2-Stellung aufweisen

${ }^{43}$ V2-Stellung ist zudem in den Komplementen von Gewissheitsprädikaten zulässig. Nach Reis (1997, 123) liegt in Sätzen wie dem folgenden allerdings Doppelpunktintonation vor, das heißt, dass der V2-Satz nicht in die FHG des vorherigen Satzes integriert ist. Aus diesem Grund werden Komplementsätze von Gewissheitsprädikaten aus den folgenden Untersuchungen ausgeklammert.

(i) Es ist KLAR: er KOmmt!

Eine weitere Gruppe an Prädikaten, welche einen V2-Satz einbetten können, bilden Präferenzprädikate (vgl. dazu Frank 2000, Meinunger 2004 und Reis 1997):

(ii) Es wäre besser, du gingest nicht hin.

Nach Meinunger (2004) weisen Konstruktionen mit Präferenzprädkaten jedoch so viele Besonderheiten auf (beispielsweise ist der Komplementsatz oder aber der Matrixsatz meistens im Konjunktiv und, falls das Prädikat wie in (ii) ein Adjektiv enthält, ist dieses gesteigert), dass es naheliegend ist, diese gesondert von den übrigen V2-Einbettern zu betrachten. Aus diesem Grund werden auch Präferenzprädikate aus den weiteren Untersuchungen ausgeklammert. 
kann wie $(207) \cdot{ }^{44}$

Jenny studiert in Athen, weil sie mag griechisches Essen.

Die Unterscheidung zwischen weil-Sätzen mit V2- und solchen mit VL-Stellung ist oft mit einem semantischen Unterschied verbunden. Mit weil-V2-Sätzen können mehr Lesarten ausgedrückt werden als mit einem kanonischen weil-VL-Satz. Während ein kausaler VL-Satz typischerweise als propositionaler Modifikator verwendet wird, werden weil-V2-Sätze häufig zum Ausdruck von epistemischen und sprechaktbezogenen Begründungen verwendet. Auf diese unterschiedlichen Lesarten wird im Folgenden jedoch nicht weiter eingegangen, für mehr Informationen vgl. u.a. Altmann (1997), Antomo/Steinbach (2010), Blühdorn (2008), Breindl (2009), Gaumann (1983), Günthner (1993), Holler (2008), Keller (1993), Küper (1991), Küper (1993), Pasch (1997), Pittner (1999), Scheffler (2008), Scheutz (1998), Uhmann (1998), Volodina (2008), Volodina (2011), Wegener (1993) und Wegener (2000).

Anders als die im vorherigen Abschnitt diskutierten V2-Komplementsätze, die mit der V2-Stellung zwar eine hauptsatztypische Eigenschaft aufweisen, sich aber dennoch wie eingebettete Sätze verhalten, sind weil-V2-Sätze eindeutig unintegriert und verhalten sich wie typische Hauptsätze. Beispielsweise zeigt Uhmann (1998), dass weil-V2-Sätze keine Quantoren-Variablen-Bindung zulassen, wie Beispiel (208) belegt, außerdem können die kausalen V2-Sätze nicht im Skopus von skopusfähigen Elementen ihres Bezugssatzes stehen, sie weisen eine separate FHG auf, sind nicht einbettbar und können Modalpartikeln und Einstellungsadverbien enthalten. Somit weisen weil-V2-Sätze alle relevanten Merkmale von unintegrierten Sätzen nach Reich/Reis (2013) auf.

(208) * Niemand $_{i}$ war verärgert, weil er $_{i}$ wurde nicht eingeladen.

Auch wenn es sich bei weil-V2-Sätzen um unintegrierte Sätze handelt, werden sie im Folgenden dennoch als Beispiel für das Auftreten von Wurzelphänomenen in einem Kontext mit den weiter oben beschriebenen V2-Komplementsätzen diskutiert. Dafür spricht zum einen, dass weil-V2-Sätze zwar unintegriert, nichtsdestotrotz aber dennoch abhängig sind: sie besitzen eine noch ungesättigte Argumentstelle und müssen diskursstrukturell mit ihrem Bezugssatz verbunden werden, um interpretierbar zu sein. Zudem treten weil-V2-Sätze nicht selbstständig auf, sondern je nach syntaktischer Analyse als Konjunkte einer koordinierten Struktur (damit würden sie nach Reich/Reis $2013 \mathrm{zu}$ den unselbstständigen Wurzelsätzen zählen) oder als CP-Adjunkte (in diesem Fall würde es sich nach Reich/Reis 2013 um unintegrier-

\footnotetext{
${ }^{44} \mathrm{Im}$ Gegensatz zu V2-Komplementsätzen entfällt die Konjunktion in weil-V2-Sätzen nicht. Daraus ergibt sich die Frage, in welcher Position weil in einem V2-Satz steht, da die $\mathrm{C}^{0}$-Position ja durch das finite Verb eingenommen wird. Eine Möglichkeit wäre, weil-V2-Sätze als Instanzen von CP-Rekursion ähnlich wie im Friesischen oder dem Dänischen, die zu den sogenannten [-Comp-drop]-Sprachen gehören, zu analysieren. Alternativ könnte es sich auch um die koordinative Verknüpfung zweier Hauptsätze handeln, wobei weil wie in Uhmann (1998) vorgeschlagen die K-Position bzw. wie in Antomo/Steinbach (2010) vorgeschlagen, die Kopfposition einer parataktischen Phrase $\pi \mathrm{P}$ einnimmt.
} 


\section{Lizenzierungskontexte}

te Nebensätze handeln). Wurzelsatztypische Eigenschaften definiert aber u.a. Holler $(2005,30)$ als solche Eigenschaften, „die man normalerweise nur in selbstständig auftretenden Sätzen beobachten kann“. Außerdem kann ein weil-V2-Satz nicht in jedem Kontext verwendet werden, in dem ein selbstständiger V2-Deklarativsatz angemessen ist. So kann ein selbstständiger V2-Deklarativsatz durchaus dafür verwendet werden, eine bereits im Kontext gegebene Information zu wiederholen (er kann also über $\mathrm{R}=$ Coreference angebunden werden), ein weil-V2-Satz ist in diesem Kontext dagegen ausgeschlossen.

A: Maria hat morgen Geburtstag.

B: Ja, Maria hat morgen Geburtstag.

B' \#Ja, ich brauche noch ein Geschenk, weil Maria hat morgen Geburtstag.

Ein weil-V2-Satz kann nur dann einen kanonischen weil-VL-Satz ersetzen, wenn spezielle Lizenzierungsbedingungen erfüllt sind. Somit unterscheidet sich das Auftreten der kausalen V2-Sätze von dem von selbstständigen V2-Sätzen. Welcher Art diese Lizenzierungsbedingungen sind, wird in Abschnitt 7 untersucht.

Die Beobachtung, dass temporale anders als kausale Adverbialsätze keine Wurzelphänomene zulassen, ist sprach- und phänomenübergreifend stabil (vgl. u.a. Andersson 1975, Haegeman 2007, Hooper/Thompson 1973 und Sawada/Larson 2004). Da die Opposition zwischen Temporal- und Kausalsätzen im Zentrum von Abschnitt III steht, soll diese Beobachtung an dieser Stelle kurz anhand einiger Beispiele belegt werden. Sawada/Larson (2004) zeigen, dass Linksversetzungen im Englischen nur in kausalen, nicht aber in temporalen Adverbialsätzen möglich sind (vgl. die Beispiele in (210)). Dasselbe gilt auch für Topikalisierungen im Schwedischen, wie aus den Beispielen in (211) ersichtlich wird.

(210) a. Mildred drives a Mercedes because her son, he owns stock in Xerox.

b. *Mildred bought a Mercedes when/before/after her son, he purchased stock in Xerox.

a. Vi foljer inte med därför att ÖIS gillar vi inte. wir folgen nicht mit weil dass ÖIS mögen wir nicht 'Wir kommen nicht mir, weil wir ÖIS nicht mögen.'

b. *Vi akte hem när Peter vi hade talat med. wir gingen heim als Peter wir hatten gesprochen $\mathrm{zu}$ 'Wir gingen heim, nachdem wir mit Peter gesprochen hatten.'

Auch für nicht-indoeuropäische Sprachen wurde festgestellt, dass temporale anders als kausale Adverbialsätze keine Wurzeleigenschaften aufweisen können. So lässt in den folgenden Sätzen aus dem Japanischen nur der Kausalsatz, nicht aber der Temporalsatz die Topikalisierung mit -wa zu (vgl. zu eingebetteten Wurzelphänomenen im Japanischen Maki et al. 1999). 
(212) a. Sake-wa karadani yoku-nai-darou-kara, oolong-cha-o

Sake-top Gesundheit gut-Neg-Mod-weil, Oolong-Tee-acc

nomo-u.

trink-werde

'Weil Sake nicht gut für die Gesundheit ist, werde ich Oolong-Tee trinken.'

b. *Taro-wa kuru-darou-mae, minna-no naka-ga

Taro-top kommt-Mod-bevor, jeder-gen relation-nom

warukunat-ta.

schlecht-werde-PST

'Bevor Taro kam, war jeder schlecht gelaunt.'

Allerdings gibt es auch Fälle, die auf den ersten Blick als Gegenbeispiele gewertet werden könnten. Beispielsweise weist der mit während eingeleitete Satz in Beispiel (213) V2-Stellung auf, nichtsdestotrotz ist der Satz grammatisch. Wie Altmann (1997) und Breindl (2009) beobachten, wird die sonst temporale Subjunktion während in diesem Fall jedoch adversativ interpretiert. Die Klammer zeigt dabei an, dass V2-Bewegung in einem adversativen während-Satz für viele Sprecher nur akzeptabel ist, wenn die erste Konstituente mit einem koreferenten Pronomen aufgegriffen wird, also eine Linksversetzung vorliegt.

(213) Sie steht jeden Morgen früh auf, während ihr Mann(, der) faulenzt den ganzen Tag.

Da V2-Stellung in Temporalsätzen nur dann möglich ist, wenn die Subjunktion adversativ interpretiert wird, können wir festhalten, dass temporale Adverbialsätze keine V2-Stellung lizenzieren. ${ }^{45}$

\subsection{Relativsätze}

Betrachten wir abschließend Sätze, die von einem nominalen Ausdruck abhängen. Wie in Teil I, in dem die Diskursfunktion von Nebensätzen mit den damit zusammenhängenden Eigenschaften untersucht wurden, ist auch bezogen auf das Vorkommen von V2-Stellung wichtig, ob der Relativsatz restriktiv oder appositiv ist, sowie ob er ein definites oder ein indefinites Antezendes modifiziert. Brandt (1990), Emonds (1970), Gärtner (2001), Gärtner (2002) und Holler (2008) beobachten nun, dass V2-Stellung nur in Relativsätzen auftreten kann, die eine restriktive Funktion ausüben. Betrachten wir dies etwas genauer. In ihrem bedeutenden Aufsatz zeigen

$\overline{{ }^{45} \mathrm{~V} 2-S t e l l u n g}$ ist zudem in Konzessivsätzen möglich, wie das folgende Beispiel belegt:

(i) Ich nehm' noch ein Bier. Obwohl: ich muss noch fahren.

Auch bei Konzessivsätzen korreliert die Alternation der Verbstellung mit einem Bedeutungsunterschied (vgl. dazu Antomo/Steinbach 2013, Günthner 1999b und Pittner 1999). Auf obwohlSätze mit V2-Stellung wird in Abschnitt 7.3.1 noch genauer eingegangen. 


\section{Lizenzierungskontexte}

Hooper/Thompson (1973), dass bei restriktiven Relativsätzen zwischen solchen mit einem definiten und solchen mit einem indefiniten nominalen Antezedens zu unterscheiden ist. Nach Gärtner (2001, 114ff) kann ein restriktiver Relativsatz mit einem definiten Antezedens nie V2-Stellung aufweisen. Ein entsprechender Satz wie (214) ist ungrammatisch (zur Begründung vgl. Gärtner 2001).

*Dominik sucht die Frau, die arbeitet in der Bibliothek.

Jedoch ist V2-Stellung in restriktiven Relativsätzen nicht grundsätzlich ausgeschlossen. Bezieht sich ein restriktiver Relativsatz auf ein indefinites Antezedens wie in Beispiel (215) nach Gärtner (2001, 98), ist V2-Stellung möglich.

Das Blatt hat eine Seite, (/) die ist ganz schwarz.

Dass es sich tatsächlich um einen nachgestellten Relativsatz mit V2-Stellung und nicht etwa um die Juxtapposition zweier Hauptsätze handelt, sieht man nach Gärtner (2001) daran, dass der nachgestellte Satz mit dem Hauptsatz, welcher mit steigender Intonation (,/') endet, eine informationsstrukturelle Einheit mit einer FHG bildet. So können V2-Relativsätze zusammen mit ihrem Bezugssatz einen All-focusSentence bilden wie in dem folgenden Beispiel aus Holler (2008, 198):

Was ist passiert?

[Anna hat ein Buch, das liest sie nicht. $]_{F}$

Zudem ist der Hauptsatz ohne den Relativsatz semantisch nicht wohlgeformt, wie Beispiel (217) belegt. Dass die Äußerung (215) im Gegensatz zu (217) semantisch wohlgeformt ist, zeigt zudem, dass der V2-Relativsatz oben eindeutig eine restriktive Funktion übernimmt und eine wichtige Rolle bei der Festlegung der Referenz des Antezedens spielt.

\#Das Blatt hat eine Seite. ( $\backslash$ Die ist ganz schwarz.

Darüber hinaus belegt Gärtner (2001) die Ansicht, dass es sich bei den vorliegenden Strukturen tatsächlich um restriktive V2-Relativsätze handelt, mit der Beobachtung, dass die Sätze sich auf das Antezedens welche, das einen restriktiven Relativsatz erfordert, beziehen können wie in dem folgenden Beispiel:

Unter den Zuschauern waren welche, die kamen freiwillig.

Außerdem führt Gärtner (2001) an, dass die V2-Strukturen durch das Indefinitpronomen eins ersetzt werden können, was ebenfalls dafür spricht, dass es sich um restriktive Relativsätze handelt, da appositive Relativsätze durch das Pronomen nicht mitersetzt werden.

(219) Hans kennt einen Bodybuilder, der mag Achternbusch, und Maria kennt auch einen. 
Auch Brandt (1990) ist der Ansicht, dass es sich bei den V2-Sätzen um restriktive Relativsätze handelt und belegt dies mit der Beobachtung, dass sich die V2-Strukturen auf prädikationale NPn beziehen können wie in (220), welche stets von restriktiven und nicht von appositiven Relativsätzen begleitet werden:

$$
\text { Maria ist ein Mensch, den sollte man nicht unterschätzen. }
$$

Wir können festhalten, dass restriktive Relativsätze mit V2-Stellung gebildet werden können, wenn sie sich auf ein indefinites Antezedens beziehen, in appositiven Relativsätzen ist V2-Stellung dagegen ausgeschlossen. Dies ist auf den ersten Blick unerwartet, da appositive Relativsätze zahlreiche andere hauptsatztypische Merkmale wie beispielsweise epistemische Partikeln aufweisen können, wie Holler (2005) zeigt. Dies zeigt, dass nicht alle Wurzelphänomene den gleichen Lizenzierungsbedingungen unterliegen.

Nach Gärtner (2001) handelt es sich bei V2-Relativsätzen auf der syntaktischen Ebene nicht um klassische Relativsätze, sondern vielmehr um Konjunkte einer parataktischen Phrase. Er argumentiert jedoch dafür, dass die V2-Strukturen semantisch gesehen Relativsätze bilden, die als satzübergreifende anaphorische Ausdrücke ein akzessibles Antezedens aufgreifen müssen, was er in DRT modelliert.

Nicht nur Definitheit, auch Spezifizität besitzt einen Einfluss auf das Auftreten von V2-Stellung in restriktiven Relativsätzen. Wir haben bereits in Abschnitt 2.2.4 gesehen, dass indefinite NPn im Deutschen ambig sind, da sie sich enweder auf einen spezifischen oder einen nicht-spezifischen Diskursreferenten beziehen können. Dabei konnte gezeigt werden, dass nur Relativsätze, die ein spezifisches Antezedens modifizieren, [+at-issue]-Inhalt ausdrücken können. Eine ganz ähnliche Beobachtung macht Gärtner (2001, 119f), der zeigt, dass restriktive Relativsätze nur dann V2Stellung aufweisen können, wenn sie sich auf einen spezifischen indefiniten Ausdruck beziehen wie in den folgenden Beispielen und in Satz (215).

(221) a. Ich kenne einen Linguisten, der kann nicht lesen.

b. Maria hat einen Fisch gefangen, der ist rot-weiß gestreift

In den folgenden beiden Beispielen nach Gärtner (2001, 119f) modifiziert der jeweilige Relativsatz dagegen eine NP mit einer nicht-spezifischen Interpretation. Wie wir sehen, ist V2-Stellung in diesem Kontext ausgeschlossen.

a. *Hans möchte einen Fisch fangen, (/) den kann er essen.

b. ${ }^{*}$ Geh zu einem Arzt, der kann das heilen.

Anders als restriktive Relativsätze können appositive dagegen grundsätzlich nicht V2-Stellung aufweisen. Aus diesem Grund ist der V2-Relativsatz in (223) nicht wohlgeformt.

(223) ??Ich habe mir ein Fahrrad gekauft, das gefällt übrigens auch Tina. 


\section{Lizenzierungskontexte}

Nicht zu verwechseln ist das Auftreten von appositiven V2-Relativsätzen mit asyndetischen Strukturen wie in (224-a) oder eingeschobenen V2-Parenthesen wie in (224-b) (vgl. dazu u.a. Steinbach 2007).

(224) a. Ich habe mir ein Fahrrad gekauft. Das gefällt übrigens auch Tina.

b. Max - den kenne ich übrigens aus Berlin - studiert seit 2003 Philosophie.

Die Abgrenzung zu tatsächlichen V2-Relativsätzen ist natürlich nicht immer leicht. Anhand von intonatorischen und informationsstrukturellen Kriterien kann jedoch gezeigt werden, dass es sich bei den Äußerungen in (224) nicht um Relativsätze handelt. Während nämlich ein V2-Relativsatz, wie bereits in (216) gezeigt wurde, zusammen mit seinem Bezugssatz eine Informationseinheit bildet und die gesamte Äußerung als Antwort auf die gegebene Frage fokussiert sein kann, ist eine solche Allfokussierung weder für asyndetische noch für parenthetische Strukturen möglich (vgl. Beispiel (225)).

Was ist passiert?

\#[Ich habe mir ein Fahrrad gekauft. Das gefällt übrigens auch Tina. $]_{F}$

Ein asyndetisch verknüpfter V2-Satz und auch eine V2-Parenthese sind immer aus der informationsstrukturellen Einheit ihres Matrixsatzes ausgeschlossen, was sich auch intonatorisch bemerkbar macht. Ein weiterer Punkt, in dem sich die Strukturen von tatsächlichen V2-Relativsätzen unterscheiden, liegt darin, dass sie ohne Einschränkung in jedem Kontext auftreten können. Die V2-Parenthese in Beispiel (224-b) beispielsweise kann auch im Vorfeld des Matrixsatzes stehen, in dieser Position sind V2-Nebensätze aber grundsätzlich ausgeschlossen, wie Beispiel (226) belegt. Dies spricht gegen eine Analyse von V2-Parenthesen als abhängige Wurzelphänomene, weswegen im Folgenden mit Emonds (1970), Gärtner (2001), Gärtner (2002) und Holler (2008) davon ausgegangen wird, dass V2-Relativsätze grundsätzlich restriktiv sind, wohingegen appositive Relativsätze keine V2-Stellung aufweisen können.

*Einen Linguisten, der kann nicht lesen, kenne ich.

Wir können festhalten, dass Relativsätze genau wie Komplement- und Adverbialsätze nicht grundsätzlich V2-Stellung zulassen. Während die Akzeptanz von V2Stellung in Komplementsätzen von der Art des einbettenden Prädikats und in Adverbialsätzen von der Wahl der nebensatzeinleitenden Subjunktion abhängt, sind für das Auftreten von V2-Stellung in Relativsätzen zwei Parameter entscheidend, nämlich (In-)Definitheit des nominalen Antezedens und (Nicht-)Restriktivität.

\subsection{Zusammenfassung des Kapitels}

In diesem Kapitel wurde das Auftreten von V2-Stellung in einigen ausgesuchten deutschen Nebensätzen untersucht. Dabei hat sich gezeigt, dass nur bestimmte Ne- 
bensatztypen V2-Stellung grundsätzlich zulassen, wohingegen V2-Stellung in den anderen Nebensatzarten ausgeschlossen ist. Eine Übersicht über die wichtigsten Lizenzierungskontexte bietet Tabelle 6.1 .

\begin{tabular}{|l|c|}
\hline Nebensatztyp & V2-Stellung \\
\hline \hline Komplementsatz, Brückenverb & $+/-$ \\
\hline Semifaktiver Komplementsatz & $+/-$ \\
\hline Faktiver Komplementsatz & - \\
\hline Temporaler Adverbialsatz & - \\
\hline Adverbialsatz mit weil & - \\
\hline Appositiver Relativsatz & - \\
\hline Restriktiver Relativsatz, definites Antezedens & - \\
\hline $\begin{array}{l}\text { Restriktiver Relativsatz, indefinites Antezedens, } \\
\text { nicht-spezifisch }\end{array}$ & + \\
\hline $\begin{array}{l}\text { Restriktiver Relativsatz, indefinites Antezedens, } \\
\text { spezifisch }\end{array}$ & + \\
\hline
\end{tabular}

Tabelle 6.1.: Lizenzierungskontexte für V2-Stellung

Von der Forschung gut untersucht ist die externe Syntax von V2-Nebensätzen, also ihre syntaktische Anbindung an den Bezugssatz, wobei diesbezüglich Konsens darüber besteht, dass V2-Nebensätze im Vergleich zu kanonisch integrierten VLNebensätzen weiter oben im Strukturbaum angefügt werden oder aber sogar syntaktische Waisen darstellen, die erst auf der Diskursebene angebunden werden. Bezogen auf V2-Komplementsätze argumentiert beispielsweise Reis (1997), dass es sich nicht um $\mathrm{V}^{0}$-Komplemente, sondern vielmehr um VP-Adjunkte handelt, wohingegen Meinunger (2004) und Truckenbrodt (2006) eine hybride Analyse mit doppelter Basisgenerierung und obligatorischer Extraposition vorschlagen. V2-Relativsätze werden von Gärtner (2001) mittels CP-Koordination beschrieben, in Gärtner (2002) revidiert er diese Analyse jedoch wieder und analysiert V2-Relativsätze als DP-Adjunkte mit obligatorischer Extraposition. Am stärksten desintegriert sind sicherlich V2Sätze mit adverbialer Funktion wie weil- und obwohl-V2-Sätze. Bezogen auf die syntaktische Beschreibung der kausalen V2-Sätze schlagen u.a. Antomo/Steinbach (2010) und Uhmann (1998) CP-Koordination vor, Antomo (2012a) und Holler (2008) beobachten dagegen, dass es sich möglicherweise sogar um komplett desintegrierte syntaktische Waisen handelt, die erst auf der Diskursebene mit ihrem Bezugssatz verknüpft werden (vgl. zur Syntax von V2-Nebensätzen auch Axel-Tober 2012, Frank 2000, Pittner 1999 und Reis 1997).

Im Folgenden wird auf Komplement-, Relativ- und weil-Sätze mit V2-Stellung dennoch weiterhin als V2-Nebensätze referiert, da diese Sätze zum einen in einer engen funktionalen Beziehung zu ihrem Bezugssatz, den sie modifizieren, stehen. Zum anderen, und dies ist weit wichtiger, unterliegt das Auftreten von V2-Stellung in den hier untersuchten Kontexten zahlreichen Bedingungen, worin sich die Sätze wesentlich von kanonischen Wurzelsätzen unterscheiden, die in jedem Kontext V2-Stellung 


\section{Lizenzierungskontexte}

aufweisen können. So sind auch Nebensätze, welche grundsätzlich V2-Stellung aufweisen können, in bestimmten Kontexten nur mit VL-Stellung wohlgeformt (für einen Überblick über die Kontexte, in denen V2-Stellung ausgeschlossen ist, vgl. Antomo/Steinbach 2010, Breindl 2009, Frank 2000, Gärtner 2001, Holler 2008, Meinunger 2004, Reis 1995, Reis 1997 und Truckenbrodt 2006). Diese mit V2-Stellung inkompatiblen Kontexte lassen sich in zwei Gruppen unterteilen: Zum einen sind V2-Nebensätze ausgeschlossen, wenn der abhängige Satz im syntaktischen Skopus eines Elements aus dem Matrixsatz steht. So blockieren V2-Relativ- und weil-Sätze c-Kommando, sie können nicht in einen Interrogativsatz integriert werden, nicht im Skopus der Matrixnegation interpretiert werden und lassen keine Korrelate zu, wobei die beiden zuletzt genannten Eigenschaften auch auf V2-Komplementsätze zutreffen. Dies zeigt, wie bereits weiter oben beschrieben, dass es sich bei V2-Nebensätzen um desintegrierte Strukturen handelt, die mit ihrem Bezugssatz entweder gar nicht oder aber an einer sehr hohen strukturellen Position syntaktisch verknüpft sind, so dass sie außerhalb des c-Kommando-Bereichs ihres Bezugssatzes stehen. Die zweite Gruppe an Kontexten, in denen V2-Nebensätze nicht auftreten können, ist semantischpragmatischer Natur. So können V2-Nebensätze grundsätzlich nicht dazu verwendet werden, eine bereits vorerwähnte Information auszudrücken. Dies kann darauf zurückgeführt werden, dass V2-Nebensätze nie präsupponiert sein dürfen - die spezifischen Lizenzierungsbedingungen von V2-Stellung in abhängigen Sätzen werden im Zentrum der folgenden Abschnitte stehen. 


\section{Lizenzierungsbedingungen}

Neben der Definition der Lizenzierungskontexte besteht ein zweites zentrales Ziel der Arbeiten zu Wurzelphänomenen darin, die ihrem Auftreten zugrundeliegenden Prinzipien zu definieren. Von der Forschung zu klären ist dabei vor allem, weshalb das Auftreten spezifischer syntaktischer und lexikalischer Phänomene auf spezielle Lizenzierungskontexte beschränkt ist und welche relevanten strukturellen oder funktionalen Eigenschaften ein solcher Kontext besitzen muss, um Wurzelphänomene zu lizenzieren. Im Wesentlichen können hier zwei grundlegend unterschiedliche Herangehensweisen unterschieden werden: ${ }^{46}$ Strukturelle Ansätze erklären das Auftreten von hauptsatztypischen Transformationen in abhängigen Kontexten primär mithilfe von syntaktischen Annahmen wie beispielsweise Beschränkungen von Bewegung. Funktionale Ansätze dagegen gehen davon aus, dass nicht-strukturelle, also semantische und pragmatische Faktoren für das Auftreten von Wurzelphänomenen verantwortlich sind. In Abschnitt 7.1 wird auf die wichtigsten funktionalen Ansätze eingegangen. Rein strukturelle Analysen, d.h. solche Analysen, die das Auftreten von Wurzelphänomenen in abhängigen Sätzen gänzlich ohne Rückgriff auf funktionale Konzepte wie beispielsweise Assertiertheit erklären, werden lediglich kurz erwähnt. Wir haben bereits in Abschnitt 5 gesehen, dass neben dem Deutschen auch andere Sprachen Wurzelphänomene in abhängigen Sätzen aufweisen können, wobei sich auch in diesen Sprachen die Frage stellt, wodurch diese hauptsatztypischen Transformationen lizenziert werden. Insbesondere die Untersuchungen zum Englischen und zu den skandinavischen Sprachen, welche ebenfalls abhängige V2-Sätze besitzen, haben dabei einen großen Einfluss auf die Erforschung von V2-Nebensätzen im Deutschen besessen. Von besonderer Bedeutung ist dabei der Aufsatz von Hooper/Thompson (1973) zum Englischen, dessen Grundgedanke in den folgenden Jahren auf zahlreiche andere Sprachen und Phänomene übertragen wurde, so auch auf abhängige V2-Sätze im Deutschen. Die in Hooper/Thompson (1973) entwickelte Assertionshypothese und deren wichtigste Adaptionen auf vor allem skandinavische Sprachen werden in Abschnitt 7.1.1 diskutiert. Ansätze, die Assertionshypothese auf das Auftreten von abhängigen V2-Sätzen im Deutschen zu übertragen, werden anschließend in Abschnitt 7.1.2 vorgestellt. Wir werden sehen, dass mit der Übertragung der ursprünglich fürs Englische entwickelten Assertionshypothese auf das Deutsche einige Probleme einhergehen, weswegen in Abschnitt 7.2 mit der At-issueness-Hypothese ein alternativer Vorschlag unterbreitet werden soll. Während die Anwendung der

\footnotetext{
${ }^{46}$ Da die meisten strukturellen Ansätze zu Wurzelphänomenen auch funktionale Aspekte enthalten und umgekehrt, ist eine dichotomische Einteilung natürlich problematisch. Dennoch lässt sich meistens eine mehr funktionale oder strukturelle Schwerpunktsetzung ausmachen, so dass die folgende Gruppierung der ersten Orientierung dienen kann.
} 


\section{Lizenzierungsbedingungen}

At-issueness-Hypothese in Abschnitt 7.2 auf die acht Nebensatztypen der Gruppen 1 und 2 beschränkt bleibt, werden in Abschnitt 7.3 weitere Anwendungsbereiche wie Konditional- und Konzessivsätze sowie die Distribution von abhängiger V2-Stellung in anderen germanischen Sprachen (Abschnitt 7.3.1), das Phänomen der eingebetteten Topikalisierung im Englischen (Abschnitt 7.3.2) und unintegrierte VL-Sätze (Abschnitt 7.3.3) untersucht. In Abschnitt 7.4 werden die Ergebnisse dieses Kapitels zusammenfasst.

\subsection{Bisherige Ansätze}

\subsubsection{Die Assertionshypothese}

Die Arbeiten von Hooper/Thompson (1973) und Kiparsky/Kiparsky (1970) stehen zu Beginn einer langen Tradition, die Lizenzierung von Wurzelphänomenen in abhängigen Sätzen rein funktional zu erklären. Grundlegend ist dabei die Taxonomie zwischen den beiden Konzepten Präsupposition und Assertion, wobei Wurzeltranformationen nach Hooper/Thompson (1973) nur in assertierten, nicht aber in präsupponierten Nebensätzen möglich sind. Wiklund et al. (2009) fassen diese Hypothese folgendermaßen zusammen:

The Assertion Hypothesis

The more asserted (the less presupposed) the complement is, the more compatible it is with V2 (and other root phenomena).

Die Assertionshypothese wurde in dieser oder leicht modifizierter Form in zahlreichen späteren Arbeiten diskutiert und auf diverse Wurzelphänomene in unterschiedlichen Sprachen übertragen. Sie fußt dabei vor allem auf der Untersuchung von Komplementsätzen und den Ergebnissen von Kiparsky/Kiparsky (1970), die zeigen, dass bestimmte Verben immer einen präsupponierten Satz einbetten. Hooper/Thompson (1973) klassifizieren nebensatzeinbettende Verben in die folgenden fünf Klassen, die sich im Wesentlichen mit den in Abschnitt 2.2.2 angenommenen Verbklassen decken (vgl. (39)).
Klasse A: strongly assertive predicates (z.B. say)
Klasse B: weakly assertive predicates (z.B. believe)
Klasse C: non-assertive predicates (z.B. doubt)
Klasse D: factive predicates (z.B. regret)
Klasse E: semifactive predicates (z.B. realize)

Hooper/Thompson (1973) untersuchen das Auftreten von zahlreichen unterschiedlichen Wurzeltransformationen im Englischen und kommen zu dem Ergebnis, dass die Verben der Klassen C und D keine Wurzelphänomene zulassen, die der Klassen A, B und E dagegen schon. Nach Hooper/Thompson (1973) selegieren die Verben der Klassen C und D ein präsupponiertes Komplement, woraus geschlossen wird, dass Wurzeltranformationen in präsupponierten Kontexten nicht lizenziert sind. Verben 
des Sagens und Meinens aber auch semifaktive Verben selegieren in der Auffassung von Hooper/Thompson (1973) dagegen Nebensätze, die Ähnlichkeiten mit direkten oder indirekten Assertionen besitzen, bzw. selbst assertiv sind. So drückt ein Satz wie (229) nach Hooper/Thompson $(1973,475)$ zwei Assertionen aus, nämlich (229-a) und (229-b):

He said it's just started to rain.

a. He said X.

b. It's just started to rain.

Daraus leiten die Autorinnen die These ab, dass eingebettete Wurzelphänomene in assertierten Kontexten lizenziert sind, eine Beobachtung, die in der Assertionshypothese in (227) zusammengefasst ist.

In den folgenden Jahren wurde die Assertionshypothese vom Englischen auf zahlreiche andere Sprachen und Phänomene übertragen. So wird beispielsweise die Distribution von Topikalisierungen mit -wa im Japanischen (vgl. dazu u.a. Maki et al. 1999 und Sawada/Larson 2004) genauso mit Rückgriff auf die Begriffe Assertion und Präsupposition erklärt wie das Auftreten von Modalpartikeln in eingebetteten Sätzen im Deutschen (siehe dazu beispielsweise Coniglio 2007). Auch die Lizenzierung von V2-Stellung in eingebetteten Sätzen im Skandinavischen wurde mithilfe der Assertionshypothese beschrieben. Beispielsweise argumentieren Julien (2007) und Wechsler (1991) bezogen auf das Schwedische und das Norwegische, dass nur Nebensätze, die ein eigenes assertives Illokutionspotential besitzen, V2-Stellung aufweisen können, wohingen die Voranstellung des finiten Verbs in präsupponierten Sätzen ungrammatisch ist. ${ }^{47} 48$

${ }^{47} \mathrm{Zu}$ Übertragungen der Assertionshypothese auf die Distribution von eingebetteter V2-Stellung im Deutschen vgl. Abschnitt 7.1.2.

${ }^{48}$ Die Assertionshypothese nach Hooper/Thompson (1973) wurde auch häufig strukturell implementiert. Aufbauend auf den Arbeiten von Rizzi (1997), denen zufolge illokutionäres Potential in einer funktionalen Projektion ForceP kodiert wird, gehen u.a. Frey (2011), Haegeman (2002) und Julien (2007) davon aus, dass nur Nebensätze, deren linke Peripherie eine eigene ForceP aufweist, Wurzeltransformationen untergehen können. Nebensätze, die über kein eigenes Illokutionspotential verfügen, haben dagegen eine reduzierte linke Peripherie, der die funktionalen Projektionen ForceP, TopP und FocP fehlen, weswegen in diesen Sätzen keine Linksversetzungen nach TopP und auch keine V2-Bewegung nach Force ${ }^{0}$ stattfinden können.

Es existieren auch rein strukturelle Ansätze, die das Auftreten von eingebetteten Wurzelphänomenen komplett ohne Rückgriff auf funktionale Konzepte wie Assertiertheit zu erklären trachten. Ein wichtiger Ansatz zur Lizenzierung von eingebetteter V2-Stellung geht auf die fundamentale Arbeit von den Besten (1983) zurück, der V2-Bewegung als die Bewegung des finiten Verbs nach $\mathrm{C}^{0}$ und die Bewegung einer anderen XP nach CSpec analysiert. Im Deutschen ist die Bewegung des finiten Verbs in die Kopfposition der $\mathrm{CP}$ blockiert, wenn $\mathrm{C}^{0}$ bereits von einem Komplementierer besetzt ist. Dementsprechend sind V2-Stellung und das Vorhandensein eines Komplementierers komplementär verteilt. Ausgehend von dieser Beobachtung schließt den Besten (1983), dass die Bewegung des finiten Verbs nach C nicht funktional motiviert ist, sondern lediglich durch die leere C-Position. Gegen eine solche Analyse spricht jedoch einiges. Zum einen weisen V2-Nebensätze in den sogenannten [-Comp-drop]-Sprachen wie dem Schwedischen oder dem Friesischen V2-Stellung ohne Wegfall des einleitenden Komplementie- 


\section{Lizenzierungsbedingungen}

Ein grundlegender Schwachpunkt der Assertionshypothese besteht darin, dass es sich bei den Begriffen Assertion und Präsupposition um Konzepte handelt, über deren genaue Definition in der Forschung kein Konsens besteht (weitere Probleme, mit denen sich die Assertionshypothese konfrontiert sieht, werden im folgenden Abschnitt diskutiert). Für eine Überprüfung der in der Assertionshypothese zusammengefassten Beobachtung ist aber eine scharfe Abgrenzung von assertierten und präsupponierten Inhalten mithilfe eindeutiger Kriterien notwendig. Allerdings haben wir bereits in Abschnitt 4 gesehen, dass mitunter sehr unterschiedliche Präsuppositionsbegriffe verwendet werden, was dementsprechend auch Konsequenzen für die Unterteilung in assertierte und präsupponierte Inhalte hat. Beispielsweise sind semifaktive Komplementsätze in der Analyse von Hooper/Thompson (1973) nicht präsupponiert und besitzen genauso wie Komplementsätze von Verben des Sagens oder Meinens Ähnlichkeiten mit direkten bzw. indirekten Assertionen. Folglich ist aufgrund der Assertionshypothese zu erwarten, dass satzförmige Komplemente von semifaktiven Verben Wurzelphänomene aufweisen können. Dies ist zutreffend, wie wir in Abschnitt 6.1 bereits bezogen auf die deutsche V2-Stellung gesehen haben (vgl. Beispiel (204), hier wiederholt als (230-b)), dasselbe gilt für Topikalisierungen im Englischen, wie Satz (230-a) aus Hooper/Thompson (1973, 480) exemplarisch belegt.

(230) a. The scout discovered that beyond the next hill stood a large fortress.

b. Ich habe gerade erfahren, ich bin durchgefallen.

Ob derartige Nebensätze allerdings tatsächlich assertiert sind, ist umstritten. So gehen beispielsweise Bentzen (2009) und Wiklund et al. (2009) davon aus, dass semifaktive Verben immer ein präsupponiertes Komplement einbetten, es sei denn, der Satz ist Teil eines Frage- oder Konditionalgefüges, in welchem Fall er dann seine Fak-

rers auf, was traditionell als CP-Rekursion analysiert wird (vgl. Beispiel (199)). Zum Teil ist das Auftreten von mit dass eingeleiteten V2-Sätzen sogar in der deutschen gesprochenen Sprache belegt (vgl. dazu Freywald 2008). Zudem bleibt die Frage ungelöst, warum nur einige Komplementsätze unter Wegfall des Komplementierers V2-Stellung aufweisen können, andere dagegen nie (Iatridou/Kroch 1992 und Vikner 1995 schlagen vor, dies lexikalische über die Selektionseigenschaften der einbettenden Prädikate zu lösen).

Ein anderer rein struktureller Ansatz wird in Haegeman (2007) und etwas jünger in Haegeman (2010) vorgestellt, wobei lediglich Topikalisierungsbewegungen in Adverbialsätzen des Englischen betrachtet werden. Die Tatsache, dass temporale und konditionale Adverbialsätze, wie in dem folgenden Beispiel dargestellt, keine Topikalisierungen aufweisen können, erklärt Haegeman $(2007,2010)$ damit, dass diese Adverbialsatztypen die Bewegung eines Operators in die linke Satzperipherie involvieren. Die Grundidee Haegemans (2007, 2010) besteht nun darin, dass diese Operatorenbewegung mit der topikalisierten Konstituente interagiert und die Ungrammatikalität des Beispiels demnach aus einem syntaktischen Interventionseffekt resultiert.

(i) *While this paper I was revising last week, I suddenly thought of another analysis.

Eine Schwachstelle dieses Ansatzes besteht darin, dass er sich auf die Erklärung eines isolierten Kontextes beschränkt, ohne das Phänomen in einen größeren Kontext einzubetten. Eine erklärungsadäquate Analyse sollte im Idealfall aber auch auf Komplement- und Relativsätze übertragbar sein. 
tivität verliert, wie bereits in Beispiel (43) in Abschnitt 2.2.2 gezeigt wurde. Dieser Auffassung zufolge sind die Komplementsätze oben präsupponiert. Da sie dennoch Wurzelphänomene aufweisen, schließen Bentzen (2009) und Wiklund et al. (2009), dass die Assertionshypothese nicht zutreffend und die Opposition von Assertion und Präsupposition für die Lizenzierung von Wurzelphänomenen in abhängigen Sätzen nicht entscheidend ist. Dieser Einwand gegen die von Hooper/Thompson (1973) aufgestellte Hypothese kann jedoch entkräftet werden. So kann an der Vorgehensweise von Bentzen (2009) und Wiklund et al. (2009) zum einen bemängelt werden, dass sie Präsuppositionen mithilfe von Projektionstests und der Wait-a-minute-Strategie ermitteln, die beide jedoch nicht zur Ermittlung von Präsuppositionen geeignet sind, wie wir in Abschnitt 3 gesehen haben. Davon einmal abgesehen kann zudem angeführt werden, dass ein unter einem semifaktiven Verb eingebetteter V2-Satz nicht über die Diskursrelation $\mathrm{R}=$ Coreference an ein Antezedens angebunden werden kann, wie das folgende Beispiel belegt. Hier greift der Komplementsatz einen im Kontext bereits gegebenen Diskursreferenten auf und, wie wir sehen, ist die Äußerung nur mit VL-Stellung wohlgeformt.

a. Oje, der arme Peter ist durchgefallen!

b. *Ja, ich habe gerade erfahren, er ist durchgefallen.

c. Ja, ich habe gerade erfahren, dass er durchgefallen ist.

Gemäß der Coreference-Regel kann daraus geschlossen werden, dass V2-Komplemente von semifaktiven Verben, anders als von Bentzen (2009) und Wiklund et al. (2009) postuliert, gerade nicht präsupponiert sind und somit auch kein Gegenbeispiel zur Assertionshypothese nach Hooper/Thompson (1973) bilden. Derartige Meinungsverschiedenheiten fußen auf der Tatsache, dass den jeweiligen Untersuchungen unterschiedliche Definitionen der Konzepte Assertion und Präsupposition zugrunde liegen. Zudem ist noch nicht vollständig klar, ob es sich bei Präsuppositionen und Assertionen um zwei komplementäre Konzepte handelt oder ob auch Bedeutungsaspekte existieren, die weder assertiert noch präsupponiert sind, und falls ja, wie sich diese bezogen auf die Lizenzierung von Wurzelphänomenen verhalten. Auch hier herrschen in der Literatur unterschiedliche Ansichten und zum Teil wird das der jeweiligen Analyse zugrundeliegende Verhältnis zwischen Assertionen und Präsuppositionen nicht explizit thematisiert.

\subsubsection{V2-Nebensätze und die Assertionshypothese}

Auch auf die Lizenzierung von V2-Stellung in deutschen Nebensätzen wurde die Grundidee der Assertionshypothese nach Hooper/Thompson (1973) übertragen. So findet sich die Ansicht, derzufolge V2-Stellung in assertierten, nicht aber in präsupponierten Nebensätzen möglich ist, in zahlreichen Arbeiten. Bezogen auf weil-Sätze nehmen beispielsweise Altmann (1997), Antomo/Steinbach (2010), Breindl (2009), Gaumann (1983), Holler (2008), Keller (1993), Küper (1993), Pasch (1997), Pittner (1999), Scheutz (1998), Uhmann (1998), Volodina (2011) und Wegener (1993) an, 


\section{Lizenzierungsbedingungen}

dass ein präsupponierter weil-Satz nur mit VL-Stellung wohlgeformt ist, während das finite Verb in einem weil-Satz, der ein eigenes assertives Illokutionspotential besitzt, in die $\mathrm{C}^{0}$-Position bewegt werden kann. Ebenso wurde das Auftreten von V2Stellung in Komplement- und Relativsätzen unter Rückgriff auf die Konzepte Assertion und Präsupposition erklärt (vgl. u.a. Gärtner 2001, Gärtner 2002, Holler 2008, Reis 1997, Scheffler 2008 und Truckenbrodt 2006). Genau wie Hooper/Thompson (1973) eine Korrelation zwischen dem Auftreten von eingebetteten Wurzelphänomenen im Englischen und der Assertivität des Satzes beobachten, ist es mittlerweile auch weitgehend Konsens, dass V2-Stellung in Nebensätzen des Deutschen mit einem assertiven Illokutionspotential zusammenhängt. Beispielsweise wird weitgehend akzeptiert, dass mit einer Äußerung wie der folgenden zwei Assertionen ausgedrückt werden können, nämlich die Gesamtäußerung (i) Peter glaubt $x$ und die mit dem Komplementsatz realisierte Assertion (ii) Maria ist in Berlin.

Peter glaubt, Maria ist in Berlin.

Aus der Übertragung der Assertionshypothese auf die deutsche V2-Stellung ergeben sich zwei mögliche Schlüsse. Es kann zum einen vermutet werden, dass es für einen abhängigen Satz ausreichend ist, nicht präsupponiert zu sein, um V2-Stellung aufweisen zu können. Dieser Schluss ist in Hypothese 1 formuliert. Eine andere mögliche Annahme besteht darin, dass nur Nebensätze, die über ein eigenes assertives Potential verfügen, auch mit V2-Stellung gebildet werden können, was in Hypothese 2 zusammengefasst ist.

\section{Hypothesen zur Lizenzierung von V2-Stellung in Nebensätzen}

H1: V2-Stellung ist nur in allen abhängigen Sätzen, die [-präsupponiert] sind, möglich.

H2: V2-Stellung ist nur in allen abhängigen Sätze, die [+assertiv] sind, möglich.

Beide Annahmen werden im Folgenden überprüft. Beginnen wir mit Hypothese 1. Dass V2-Stellung nicht in präsupponierten Nebensätzen möglich ist, lässt sich daran erkennen, dass ein V2-Nebensatz nie vorerwähnt sein darf (vgl. dazu u.a. Antomo/Steinbach 2010, 7, Breindl 2009, 11, Gaumann 1983, Keller 1993 und Pasch et al. 2003). Aus diesem Grund ist die Äußerung des weil-V2-Satzes in Beispiel (234-a) nach Breindl $(2009,11)$ nicht wohlgeformt, dasselbe gilt für den argumentrealisierenden V2-Satz in (234-b).

a. \#Hans hat verloren. Wir haben schon befürchtet, dass er zu WEInen anfängt, weil er hat verloren.

b. \#Susanne hat gestern abgeschrieben. Der Dozent hat all seinen Kollegen erzählt, sie hat abgeschrieben.

In Kapitel 4 wurde dafür argumentiert, dass Ausdrücke, die eindeutig [-präsupponiert] sind, nicht über die Relation $\mathrm{R}=$ Coreference an ein Antezedens angebunden 
werden können, und diese Beobachtung wurde in der Coreference-Regel zusammengefasst (vgl. (178)). Die Tatsache, dass ein V2-Nebensatz nicht wohlgeformt ist, wenn sein Inhalt bereits vorerwähnt ist, zeigt demnach, dass V2-Nebensätze keine präsupponierte Lesart erlauben. Was daraus aber noch nicht gefolgert werden kann, ist, dass alle Nebensätze, die nicht präsupponiert sind, auch V2-Stellung zulassen, was Inhalt von Hypothese 1 ist. Tatsächlich lässt sich Hypothese 1 leicht widerlegen. Nimmt man an, dass das Auftreten von V2-Stellung in allen deutschen Nebensätzen, die nicht präsupponiert sind, lizenziert ist, ergibt sich mindestens ein Fall, in dem keine korrekte Vorhersage gemacht wird. In Abschnitt 6.3 wurde gezeigt, dass appositive Relativsätze grundsätzlich nur mit VL-Stellung wohlgeformt sind (vgl. dazu Beispiel (223)). Gleichzeitig aber haben wir bereits in Abschnitt 3.1.1 gesehen, dass die Verwendung eines appositiven Relativsatzes in einem Kontext, in dem der in ihm ausgedrückte Inhalt bereits gegeben ist, nicht wohlgeformt ist, wie die Beispiele in (96) aus Abschnitt 3.1.1, hier wiederholt als (235), belegen (Beispiel (235-a) nach Holler $(2005,54))$.

$$
\begin{aligned}
& \text { a. \#Ein Mann küsste eine Frau und diese Frau, die er übrigens küsste, war } \\
& \text { verheiratet. } \\
& \text { b. Jap ist gerade in Indonesien. \#Jap, der (übrigens) gerade in Indone- } \\
& \text { sien ist, fährt gerne ans Meer. }
\end{aligned}
$$

Aus der Beobachtung, dass ein appositiver Relativsatz nie durch die Diskursrelation $\mathrm{R}=$ Coreference an ein koreferentes Antezedens angebunden werden kann, folgt, dass appositive Relativsätze grundsätzlich keine präsupponierte Lesart zulassen, eine Ansicht, die auch schon von Holler $(2005,54)$ und Hooper/Thompson $(1973,487)$ formuliert wurde. Demnach können wir davon ausgehen, dass Hypothese 1 nicht zutreffend ist, da mit appositiven Relativsätzen ein Nebensatztyp vorliegt, der eindeutig [-präsupponiert] ist und dennoch keine V2-Stellung aufweisen kann.

Wie sieht es nun mit Hypothese 2 aus? Um zu überprüfen, ob V2-Stellung in deutschen Nebensätzen durch ein assertives Illokutionspotential lizenziert wird, stellt sich die Frage, auf welche Weise beurteilt werden kann, ob ein abhängiger Satz assertiv ist. Genauer gesagt: Auf welche Weise kann gezeigt werden, dass ein V2-Nebensatz wie der Komplementsatz in Beispiel (232) oder ein Relativ- bzw. weil-Satz mit V2Stellung tatsächlich über ein eigenes assertives Potential verfügt? In der Forschungsliteratur wird die Existenz eines assertiven Illokutionspotentials auf unterschiedliche Weisen belegt. Beginnen wir mit der Beobachtung, dass V2-Nebensätze typischerweise nicht in den Matrixsprechakt integiert sind. Beispielsweise kann der nachgestellte Kausalsatz in der folgenden Äußerung nach Uhmann $(1998,121)$ nicht Teil der Frageäußerung sein, sondern drückt vielmehr eine eigene Assertion aus, welche als sprechaktbezogene Begründung interpretiert wird.

(236) Kannst du mir bitte mal die Soße reichen? Weil mein Arm ist nicht so lang.

Auch Relativsätze mit V2-Stellung können nicht Teil eines Interrogativsatzes sein, wie das folgende Beispiel belegt. Anders als weil-V2-Sätze können V2-Relativsätze 
jedoch nicht außerhalb des Matrixsprechaktoperators interpretiert werden, weshalb die Äußerung ungrammatisch ist.

(237) *Kennst du einen Linguisten, der kann nicht lesen?

Die Beobachtung, dass Relativ- und weil-Sätze mit V2-Stellung nicht in den Matrixsprechakt integriert werden können, deuten u.a. Antomo/Steinbach (2010), Gärtner (2001), Holler (2008), Küper (1991), Pasch (1997), Uhmann (1998) und Wegener (1993) als Indiz dafür, dass die nachgestellten V2-Sätze über ein eigenes Assertionspotential verfügen. V2-Komplementsätze verhalten sich nun allerdings anders als Relativ- und weil-Sätze mit V2-Stellung. So belegt das folgende Beispiel, dass ein argumentrealisierender V2-Satz problemlos in einen Interrogativsatz integriert werden kann.

Hat Peter geglaubt, Maria war auf der Party?

Diese Beobachtung aber legt nahe, dass mit einem V2-Komplementsatz keine selbstständige Assertion ausgedrückt wird. Stattdessen wird in diesem Kontext häufig von einem proto-assertiven Potential ausgegangen, worauf weiter unten noch genauer eingegangen wird.

Ein weiterer Test, um zu überprüfen, ob ein abhängiger Satz über ein eigenes Illokutionspotential verfügt, betrifft dessen Integration in die FHG des Hauptsatzes. Strukturen bestehend aus einem Matrix- und einem integrierten Nebensatz können nach Brandt (1990), Holler $(2008,196)$ und Reis $(1997,128)$ zusammen eine FHG bilden, wie in dem folgenden Beispiel dargestellt.

Was ist passiert?

[Peter musste den Preis zurückgeben, weil er geSCHUmmelt hat.. $]_{F}$

Desintegrierte Sätze dagegen, mit deren Äußerung ein eigener Sprechakt realisiert wird, sind nicht in die FHG ihres Bezugssatzes integriert. Aus diesem Grund ist das folgende Beispiel zumindest stark markiert.

Was ist passiert?

?[Peter musste den Preis zurückgeben, weil er hat geSCHUmmelt. $]_{F}$

Ein weil-V2-Satz weist nach u.a. Altmann $(1997,75)$ vielmehr eine getrennte FHG mit einem eigenem Fokus auf, wie in der folgenden Struktur dargestellt.

Peter musste den Preis zuRÜCKgeben, ( $\backslash$ ) weil er hat geSCHUmmelt.

Dass weil-V2-Sätze eine eigene informationsstrukturelle Einheit bilden, spricht ebenfalls dafür, dass mit den V2-Kausalsätzen eigenständige Illokutionen realisiert werden. Anders als weil-V2-Sätze sind Komplement- und Relativsätze mit V2-Stellung dagegen informationsstrukturell in ihren Matrixsatz integriert, wie u.a. Gärtner (2001), Gärtner (2002) und Reis (1997) beobachten. Aus diesem Grund sind die folgenden beiden Beispiele, in denen Haupt- und V2-Nebensatz jeweils zusammen 
eine FHG aufweisen, vollkommen wohlgeformt. Dies deutet darauf hin, dass weder V2-Relativ- noch V2-Komplementsätze eigenständige Assertionen bilden.
a. Was ist passiert?
[Peter kennt einen Linguisten, der kann nicht LEsen. $]_{F}$
b. Was ist passiert?
[Peter hat erzählt, Maria hat wieder angefangen zu RAUchen. $]_{F}$

Auch das Auftreten von Modalpartikeln, Einstellungsadverbien und Performanzanzeigern wie hiermit gilt nach u.a. Coniglio (2007), Haegeman (2002), Holler (2008, 196), Jacobs (1986), Ormelius-Sandblom (1997), Reis (1997, 128) und Thurmair (1989) als Indikator für ein illokutionäres Potential. Zentral ist, dass die Nebensatztypen, welche im Deutschen mit V2-Stellung gebildet werden können, typischerweise auch Modalpartikeln zulassen, wohingegen die V2-Blockierer auch mit Modalpartikeln nicht wohlgeformt sind, wie die Beispiele $(243-\mathrm{a})$ nach Borst $(1985,114)$ und (243-b) nach Thurmair $(1989,109)$ für argumentrealisierende Sätze belegen (vgl. für einen Überblick über das Auftreten von Modalpartikeln in abhängigen Sätzen Coniglio 2007).

(243) a. Ich denke, dass wir das Problem schon lösen werden.

b. *Er leugnete, dass er die Zeugin ja unter Druck gesetzt habe.

Auch für Adverbialsätze gilt, dass weil-Sätze, welche ja auch mit V2-Stellung gebildet werden können, Modalpartikeln zulassen (vgl. Beispiel (244-a)), Temporalsätze dagegen nicht, wie Beispiel (244-b) nach Kratzer $(1999,5)$ zeigt.

(244) a. Weil sie ja immer zu spät kommt, habe ich heute für sie ein Taxi bestellt.

b. *Als ich ja in Syracuse gewohnt habe, war ich oft in Ithaca.

Auch die entsprechenden V2-Strukturen können eine Modalpartikel enthalten:

(245) a. Ich denke, wir werden das Problem schon lösen.

b. Ich habe heute ein Taxi für sie bestellt, weil sie kommt ja immer zu spät.

Problematisch bezogen auf Hypothese 2 ist nun aber die Tatsache, dass auch appositive Relativsätze Ausdrücke enthalten können, welche typischerweise auf assertive Wurzelsätze beschränkt sind. Dies belegt Reis $(2006,373)$ mit Beispiel (246-a) und auch Thurmair $(1989,80)$ zeigt anhand von Beispiel (246-b), dass das Einfügen einer Modalpartikel einen ansonsten ambigen Relativsatz disambiguiert, indem er nur noch appositiv interpretiert werden kann (vgl. dazu auch Coniglio 2007, 133f und Holler 2005, 74).

(246) a. Tom, der ja seit Jahren arbeitslos ist, hat sich auch beworben.

b. Autos, die (ja) laut sind, sollten mit einer geschlossenen Motorkapsel versehen werden. 


\section{Lizenzierungsbedingungen}

Obwohl appositive Relativsätze aber sprecherorientierte Ausdrücke enthalten können, werden sie im Deutschen nie mit V2-Stellung gebildet. Auch das Verhalten von restriktiven Relativsätzen in Verbindung mit Modalpartikeln legt nahe, dass Hypothese 2 das Auftreten von V2-Stellung im Deutschen nur bedingt erklären kann. So wurde schon häufig beobachtet, dass restriktive Relativsätze grundsätzlich keine sprecherorientierten Ausdrücke wie Modalpartikeln zulassen, wie aus den folgenden Beispielen ersichtlich wird (Beispiel (247-a) nach Zimmermann 2004, 32).

a. *Die Firma sucht einen Angestellten, der ja immer pünktlich ist.

b. *Ich erwarte eine Kollegin, die nämlich in Hamburg wohnt.

Dies zeigt, dass die abhängigen Sätze über kein eigenes assertives Potential verfügen, sondern vielmehr in die Matrixillokution integriert sind. Dennoch kann ein restriktiver Relativsatz, zumindest wenn er ein indefinites Antezedens modifiziert, V2Stellung aufweisen. Beispielsweise kann der Relativsatz in (247-b) unter Wegfall der Modalpartikel in einen V2-Satz umgewandelt werden. Umgekehrt können wir feststellen, dass Relativsätze mit V2-Stellung genau wie ihre VL-Gegenstücke keine sprecherorientierten Ausdrücke zulassen (vgl. Beispiel (248-b)).

a. Ich erwarte eine Kollegin, die wohnt in Hamburg.

b. ?Das Buch hat eine Seite, (/) die ist ja ganz schwarz.

Ein weiterer Test zur Überprüfung, ob ein Nebensatz ein eigenes Illokutionspotential besitzt, involviert Widerspruchsstrategien. Klassischerweise wird davon ausgegangen, dass nur assertierte Inhalte von einem direkten Widerspruch betroffen sind. Wir haben aber bereits in Abschnitt 3.2 gesehen, dass auf diese Weise nicht assertierte von präsupponierten Inhalten unterschieden werden, sondern vielmehr [+at-issue]von [-at-issue]-Inferenzen. Handelt es sich nun aber um eine direkte Zurücknahme durch den Sprecher selbst, erhalten wir andere Ergebnisse. So beobachtet u.a. Keller (1993, 231), dass sich ein Sprecher nur schwer vom Inhalt eines weil-V2-Satzes distanzieren kann (Bewertung nach Keller 1993, 231).

(249) *Er ist nach Hause gegangen, weil er hatte Kopfweh. Aber ich glaube nicht, dass das sein wirklicher Grund war.

Dass dem Inhalt des Kausalsatzes nicht durch denselben Sprecher widersprochen werden kann, kann darauf zurückgeführt werden, dass es sich um eine Assertion handelt. Die Besonderheit eines assertiven Sprechakts besteht nach Searle (1969) gerade darin, dass S an das Zutreffen der ausgedrückten Proposition glaubt (vgl. dazu auch Julien 2007 und Wiklund et al. 2009). Auch die Zurücknahme eines V2-Relativsatzes durch den Sprecher selbst ist fragwürdig (vgl. Beispiel (250-a)), wohingegen sich ein Sprecher ohne Probleme vom Inhalt eines V2-Komplementsatzes distanzieren kann, wie Beispiel (250-b) belegt.

a. ?Peter kennt einen Linguisten, der kann nicht lesen. Aber ich glaube nicht, dass er tatsächlich Analphabet ist. 
b. Peter glaubt, Maria hat das Armband gestohlen. Aber ich glaube nicht, dass sie das wirklich getan hat.

Wir können festhalten, dass sich V2-Nebensätze nur teilweise wie Assertionen verhalten. Während weil-V2-Sätze alle vier der hier untersuchten typischen Merkmale von Assertionen aufweisen, trifft dies nicht auf die anderen V2-Nebensätze zu. Komplementsätze mit V2-Stellung können in einen Interrogativsatz integriert werden und sind Teil der FHG ihres Bezugssatzes, zudem kann ihrem Inhalt durch den Sprecher selbst widersprochen werden. Dies zeigt, dass mit einem V2-Komplementsatz kein eigenständiger assertiver Sprechakt realisiert wird. Dasselbe gilt für Relativsätze mit V2-Stellung, welche ebenfalls informationsstrukturell in ihren Bezugssatz integriert sind und zudem keine Modalpartikeln enthalten können. Lediglich weil-V2-Sätze verhalten sich in allen untersuchten Punkten wie Assertionen.

Auch Gärtner/Michaelis (2010) zeigen, dass die Modellierung von abhängiger V2Stellung mithilfe der Assertionshypothese im Deutschen problematisch ist. Ausgehend von der Annahme, dass sich ein Sprecher S mit der Assertion von $\Phi$ auf $\Phi$ festlegt (abgekürzt als $\vdash_{S} \Phi$ ), bilden die Sätze in (251) Gegenbeispiele zu der Annahme, V2-Stellung sei nur in Assertionen möglich. So entspricht das Matrixsubjekt in (251-a) nicht dem Sprecher, dementsprechend legt sich S mit der Äußerung des Satzes nicht auf die abhängige Proposition fest, und in Beispiel (251-b) ist der V2Komplementsatz von einem Präferenzprädikat selegiert.

a. Mein Onkel behauptet, heute schneit es in Berlin.

b. Es ist besser, du gehst jetzt.

Zudem machen Gärtner/Michaelis (2010) auf einen weiteren Fall aufmerksam, der problematisch bezogen auf die Assertionshypothese ist. Werden zwei V2-Sätze wie in Beispiel (252) disjunktiv miteinander koordiniert, legt sich der Sprecher auf keines der beiden Konjunkte fest, es gilt also $\nvdash_{S} \Phi$ und $\nvdash_{S} \Phi$. Somit liegen mit derartigen Beispielen ebenfalls Fälle vor, in denen V2-Stellung nicht mit einem assertiven Potential korreliert.

[In Berlin schneit es] $\Phi$ oder [in Potsdam scheint die Sonne] $\Psi$.

Wir haben gesehen, dass die Assertionshypothese bezogen auf das Auftreten von V2-Stellung in abhängigen, aber z.T. auch in selbstständigen Sätzen, mit einigen Problemen verbunden ist. Insbesondere Komplement- und Relativsätze mit V2Stellung weisen nicht alle typischen Eigenschaften einer Assertion auf. Um dieses Problem zu lösen, wird in diesem Kontext häufig von proto-assertiven Äußerungen gesprochen (vgl. beispielsweise Gärtner 2002, Holler 2008 und Truckenbrodt 2006). Nach Gärtner $(2002,40)$ bezeichnet Proto-Assertivität ein schwächeres Konzept als Assertivität. Gärtner $(2002,40)$ geht davon aus, dass Proto-Assertivität eine Vorstufe der Assertivität darstellt und sich je nach Kontext, in dem sie auftritt, anders verhält. Während aus Proto-Assertivität in selbstständigen Sätzen Assertivität entsteht, wird sie in abhängigen Kontexten absorbiert. In V2-Komplementsätzen 


\section{Lizenzierungsbedingungen}

wird das proto-assertive Potential nach Gärtner $(2002,40)$ durch das Matrixprädikat aufgenommen, weswegen ein V2-Komplementsatz auch in einen Interrogativsatz integriert werden kann, ohne dass dies zu einer ungrammatischen Struktur führt. Bei V2-Relativsätzen dagegen absorbiert das assertive Illokutionspotential des Bezugssatzes die proto-assertive Kraft des abhängigen Satzes. Aus diesem Grund kann ein V2-Relativsatz auch nicht in einen Interrogativsatz integriert werden, da sein proto-assertives Potential in diesem Fall nicht absorbiert werden kann. Was weilSätze mit V2-Stellung betrifft, argumentieren Antomo/Steinbach (2010, 22f), dass das proto-assertive Potential in diesem Fall nicht absorbiert wird und folglich mit jedem weil-V2-Satz eine eigenständige Assertion realisiert wird. Aus diesem Grund können weil-V2-Sätze auch als sprechaktbezogene Begründungen einen Interrogativsatz modifizieren, da ihr proto-assertives Potential nicht absorbiert werden muss, sondern in Assertivität resultiert.

Eine etwas formalere Ausarbeitung des Konzeptes der Proto-Assertion liefert Truckenbrodt (2006). Truckenbrodt (2006) entwickelt eine derivationelle Ausarbeitung der häufig formulierten Intuition, dass die Bewegung des finiten Verbs nach $\mathrm{C}$ im Deutschen mit der Zuweisung von illokutionärem Potential zusammenhängt, wobei er die assertive Semantik von V2-Sätzen kompositionell aus den einzelnen Formmerkmalen des Satzes abzuleiten versucht (vgl. für einen ähnlichen Ansatz auch Bayer 2004, Brandner 2004, Gärtner 2001, Lohnstein 2000 und Lohnstein/Bredel 2004). Nach Truckenbrodt (2006) interagieren grammatische Merkmale in C mit spezifischen Interpretationen. Genauer gesagt nimmt Truckenbrodt (2006) die beiden Kontextindizes $<$ Deont $_{S}(, \mathrm{x})_{1}>$ und $<$ Epist $_{2}>$ an:

$$
\begin{aligned}
& <\operatorname{Deont}_{S}(, \mathrm{x})_{1}>\quad \text { 'S wants from } A \text { ' } \\
& <\text { Epist }_{2}>
\end{aligned}
$$

Mit der Ausnahme von rein exklamativen Äußerungen ist der Kontextindex $<$ Deont $_{S}$ $(, x)>$ in allen selbstständigen Sätzen aktiv. So sind alle Sprechakte auf irgendeine Weise volitional bzw. deontisch, indem sie ein Begehren von S ausdrücken. Mit der Äußerung eines Imperativsatzes wie (254-a) möchte S den Adressaten dazu veranlassen, eine Handlung auszuführen. Mit der Äußerung eines Deklarativsatzes möchte S dagegen den Wissenshintergrund von A und mit der Äußerung eines Interrogativsatzes seinen eigenen epistemischen Zustand verändern. Eine Frage wie (254-b) drückt demnach aus, dass S etwas von A wissen möchte, während S mit der Äußerung der Assertion in (254-c) A Wissen mitteilen möchte.
a. Lies das Buch!
$S$ möchte von A, dass A das Buch liest.
b. Hat Tim das Buch gelesen?
$S$ möchte von A, dass es Common Ground ist, ob Tim das Buch gele- sen hat.
c. Tim hat das Buch gelesen.
$S$ möchte von A, dass es Common Ground ist, dass Tim das Buch 
gelesen hat.

Während der Kontextindex $<\operatorname{Deont}_{S}(, \mathrm{x})_{1}>$ in allen selbstständigen Sätzen (außer in Exklamativsätzen) aktiv ist, hängt die Präsenz von $<$ Epist $_{2}>$ von grammatischen Merkmalen in C ab. Wie wir an den Beispielen in (254) sehen können, führen nur der Deklarativ- und der Interrogativsatz zu einem Common Ground-Update, wohingegen $<$ Epist $_{2}>$ in einem Imperativsatz nicht aktiv ist. Daraus leitet Truckenbrodt (2006) die Regel ab, dass $<$ Epist $_{2}>$ im Deutschen nur aktiv ist, wenn C ein [+wh]Merkmal oder aber ein finites Verb im Indikativ oder Konjunktiv II enthält. ${ }^{49}$ In einem Deklarativsatz ist die Präsenz des finiten Verbs in $\mathrm{C}^{0}$ der einzige Auslöser für $<$ Epist $_{2}>$. Dies wird an dem folgenden syntaktischen Minimalpaar nach Reis (2006, 371) deutlich. Mit der Äußerung des V2-Satzes (255-a) realisiert S eine Assertion, die den Common Ground um die genannte Information p erweitert und alle mit $\mathrm{p}$ inkompatiblen Welten aus dem Kontextset löscht. Dagegen kann der selbstständige VL-Satz in (255-b) zu keiner Common Ground-Veränderung führen, sondern nur exklamativ oder, in Abhängigkeit der Modalpartikel ja, direktiv interpretiert werden.

a. Alle sind rechtzeitig da.

b. Dass (ja) alle rechtzeitig da sind!

In Interrogativsätzen ist $<$ Epist $_{2}>$ dagegen unabhängig von der Verbstellung aktiv. So drücken sowohl der V1-Satz (256-a) als auch der ob-VL-Satz in (256-b) den Wunsch von S nach einer Common Ground-Veränderung aus. Daraus schließt Truckenbrodt (2006), dass $<$ Epist $_{2}>$ in beiden Fällen durch das [+wh]-Merkmal in C aktiviert wird, in V1-Interrogativsätzen wie (256-a) sowie in w-V2-Interrogativsätzen wird $<$ Epist $_{2}>$ zudem zusätzlich durch die Position des finiten Verbs in $\mathrm{C}^{0}$ lizenziert.

a. Kommt er?

b. Ob er wohl kommt?

Den semantischen Unterschied zwischen dem ob-VL-Satz (256-b) und einem V1Fragesatz wie (256-a) erfasst Truckenbrodt (2006) durch eine Erweiterung des Kontextindexes $<$ Deont $_{S}>$ um die zusätzliche Spezifizierung 'from A'. So beobachtet Truckenbrodt (2006), dass nur Sätze, in denen die Kopfposition der CP durch ein finites Verb besetzt ist, eine Reaktion des Adressaten präsupponieren. Der Parameter 'from A' ist demnach nur in V-in-C-Sätzen aktiviert. Aus diesem Grund ist mit der Äußerung des V1-Fragesatzes (256-a) eine Antworterwartung verbunden, mit der des VL-Satzes (256-b) dagegen nicht. Analog dazu besteht die erwünschte Reaktion von A nach der Äußerung eines V2-Deklarativsatzes, die ausgedrückte Information p zu akzeptieren. A kontrolliert folglich, ob die gewünschte CommonGround-Erweiterung eintritt oder nicht. Die Interaktion der beiden Kontextindizes

\footnotetext{
${ }^{49}$ Ein finites Verb im Konjunktiv I dagegen führt zu keiner Common Ground-Erweiterung, sondern
} zur Herstellung eines Zustands, wie das folgende Beispiel belegt:

$$
\mathrm{X} \text { sei eine Primzahl. }
$$


$<$ Deont $_{S}(, \mathrm{x})_{1}>$ und $<$ Epist $_{2}>$ mit grammatischen Merkmalen in C sind in (257) nach Truckenbrodt $(2006,265)$ zusammengefasst:

In a context index $<\operatorname{Deont}_{S}(, \mathrm{x})(,<$ Epist $>)>$ in $\mathrm{C}$

a. Epist is present iff

(i) C contains a finite verb with indicative or Konjunktiv II or

(ii) $\mathrm{C} / \mathrm{CP}$ is marked $[+\mathrm{WH}]$.

b. $\mathrm{x}=\mathrm{A}($ ddressee $)$ iff $\mathrm{C}$ contains a finite verb with person inflection.

Ein deutscher V2-Deklarativsatz hat demzufolge die in (258) dargestellte Strukturbedeutung:

$$
\begin{aligned}
& <<\text { Deont }_{S}, \mathrm{~A}>,<\text { Epist }>> \\
& \text { 'S wants from A that it is Common Ground that } p \text { ' }
\end{aligned}
$$

Zentral ist nun, dass Truckenbrodt (2006) seine Analyse auch auf abhängige V2Stellung überträgt, indem er argumentrealisierende V2-Sätze betrachtet. Auch in V2-Nebensätzen korreliert die Besetzung von $\mathrm{C}^{0}$ durch ein finites Verb mit der Aktivierung des Kontextindexes $<$ Epist $_{2}>$. Im Unterschied zu Wurzelsätzen ist dagegen der Index $<\operatorname{Deont}_{S}(, \mathrm{X})>$ nach Truckenbrodt $(2006,281)$ in abhängigen Sätzen nicht aktiv, da S und A nur auf der Matrixebene zur Verfügung stehen. Ein Komplementsatz mit V2-Stellung verfügt demnach über eine epistemische Komponente, ohne jedoch zu einem Common Ground-Update zu führen. Dies deckt sich mit der Intuition, dass S mit einer Äußerung wie der folgenden nicht generell beabsichtigt, den Inhalt des Nebensatzes dem Common Ground hinzuzufügen.

$$
\text { Peter glaubt, Maria hat wieder mit dem Rauchen angefangen. }
$$

Der semantische Beitrag der epistemischen Komponente eines V2-Nebebsatzes besteht nach Truckenbrodt (2006) darin, dass $<$ Epist $_{2}>$ nicht kompatibel mit zur Äußerung vorhergehendem Wissen um die im Nebensatz ausgedrückte Proposition $\mathrm{p}$ ist. Dies bedeutet, dass ein V2-Nebensatz keine bereits im Common Ground enthaltene Information ausdrücken darf. Somit hat der in Truckenbrodt (2006) vorgeschlagene Ansatz mehr Ähnlichkeiten mit Hypothese 1 als mit Hypothese 2 und stößt auf die weiter oben angesprochenen Probleme.

Hypothese 2 ist bezogen auf das Auftreten von abhängiger V2-Stellung im Deutschen folglich nur dann zutreffend, wenn das eigentlich binäre Konzept der Assertivität um die schwächere Kategorie der Proto-Assertivität erweitert wird. Ein solch graduelles Verständnis von Assertivität ist aber fragwürdig. Die wesentlichen Merkmale einer Assertion bestehen nach u.a. Searle (1969) und Stalnaker (1978) darin, dass S die geäußerte Proposition p glaubt, sich auf die Wahrheit von p festlegt und p dem Common Ground hinzufügen will. Beispielsweise hängt mit der Äußerung des folgenden Satzes die Aufforderung an A zusammen, $\mathrm{p}=$ Maria hat das Armband gestohlen zu akzeptieren und alle mit p inkompatiblen Welten aus dem Kontextset zu löschen. 
Vor diesem Hintergrund stellt sich nun die Frage, worin genau die Funktion einer proto-assertiven Äußerung besteht. Weist ein Komplementsatz V2-Stellung auf, wie in dem folgenden Beispiel, signalisiert $\mathrm{S}$ keineswegs, dass er an das Zutreffen der abhängigen Proposition glaubt, und will im Normalfall auch nicht, dass sie dem CG hinzugefügt wird. Vielmehr signalisiert S mit (261), dass er die Proposition Peter glaubt $x$ für wahr hält und sich nur bedingt auf die Wahrheit des Nebensatzes festlegen will.

(261) Peter glaubt, Anna hat das Armband gestohlen.

Dann aber ist unklar, worin sich proto-assertive von assertiven Äußerungen unterscheiden. Aus diesem Grund wird Assertivität im Folgenden weiterhin als nichtgraduelle Kategorie ohne Verwendung des Konzepts der Proto-Assertivität betrachtet. Dieser Auffassung zufolge kann mit einem Satz entweder eine eigene Illokution realisiert werden oder aber nicht, ohne dass es dazu Zwischenstufen gibt. Ausgehend von einem solchen nicht-graduellen Verständnis von Assertivität und Illokutionspotential ist die Assertionshypothese auch in ihrer in Hypothese 2 formulierten Ausprägung nicht haltbar. ${ }^{50}$

Fassen wir die Ergebnisse dieses Abschnitts kurz zusammen. Es wurde davon ausgegangen, dass die Assertionshypothese nach Hooper/Thompson (1973) auf zwei unterschiedliche Weisen auf die Lizenzierung von V2-Stellung in deutschen Nebensätzen übertragen werden kann. Eine Möglichkeit, die in Hypothese 1 genannt wurde, besteht darin, dass alle Nebensätze, die [-präsupponiert] sind, V2-Stellung aufweisen können. Richtig ist, dass alle V2-Nebensätze [-präsupponiert] sind, ein Schluss, zu dem auch Truckenbrodt (2006) im Rahmen seines derivationellen Ansatzes kommt. Da mit appositiven Relativsätzen aber Strukturen vorliegen, die eindeutig [-präsupponiert] sind und dennoch nie V2-Stellung aufweisen, scheint es sich bei dem Merkmal [-präsupponiert] nur um ein notwendiges, aber um kein hinreichendes Kriterium für V2-Nebensätze zu handeln. Geht man dagegen, wie in Hypothese 2 formuliert, davon aus, dass nur Nebensätze, die [+assertiv] sind, mit V2-Stellung gebildet werden können, muss berücksichtigt werden, dass sich Komplement- und Relativsätze mit V2-Stellung nicht wie typische Assertionen verhalten. Ob ein Satz assertiv ist, wurde dabei anhand von vier typischen Eigenschaften überprüft, und zwar der Möglichkeit, den Satz in einen Interrogativsatz zu integrieren, dem Auftre-

\footnotetext{
${ }^{50}$ Ein weiteres Problem, das sich aus der Annahme von Hypothese 2 ergibt, betrifft die Beobachtung, dass Assertivität (oder Proto-Assertivität) nicht auf V2-Sätze beschränkt ist, worauf im folgenden Abschnitt noch genauer eingegangen wird. So beobachtet Holler (2008, 202), dass auch freie dass-Sätze Ähnlichkeiten mit Assertionen aufweisen, zudem zeigt Reis (2013), dass sich desintegrierte weil-Sätze mit VL-Stellung wie weil-V2-Sätze verhalten und beispielsweise ebenfalls als Sprechaktbegründungen verwendet werden können. Auch Frey (2011) argumentiert, dass auch Komplementsätze mit VL-Stellung ein eigenes illokutionäres Potential, kodiert in einer ForceP, besitzen können, was sich dann beispielsweise in dem Auftreten von Modalpartikeln äußert.
} 


\section{Lizenzierungsbedingungen}

ten von sprecherorientierten Ausdrücken, der informationsstrukturellen Integration und der direkten Zurücknahme durch den Sprecher selbst.

Die Diskussion in diesem Abschnitt hat gezeigt, dass das Auftreten von V2-Stellung in deutschen Nebensätzen mithilfe der Konzepte Präsupposition und Assertion nur bedingt erklärt werden kann. Aus diesem Grund werde ich im folgenden Abschnitt dafür argumentieren, dass eine andere Kategorie für die Lizenzierung von V2-Stellung im Deutschen verantwortlich ist, nämlich At-issueness.

\subsection{V2-Stellung als grammatische Markierung für At-issueness}

Die Übertragung der Assertionshypothese auf die Lizenzierung von V2-Stellung in abhängigen Sätzen des Deutschen ist mit einigen Problemen verbunden, da die Anwendung je nach Auslegung entweder zu restriktiv oder nicht restriktiv genug ist. Wird davon ausgegangen, dass alle [-präsupponierten] Nebensätze V2-Stellung zulassen, kann nicht erklärt werden, dass appositive Relativsätze nicht mit V2-Stellung gebildet werden, die Regel ist demnach nicht restriktiv genug. Die Annahme, derzufolge nur Nebensätze mit einem eigenem assertiven Illokutionspotential V2-Stellung aufweisen können, ist dagegen zu restriktiv, da weder Komplement- noch Relativsätze mit V2-Stellung illokutionär selbstständig sind, wie im letzten Abschnitt gezeigt wurde. Aus diesem Grund soll im Folgenden dafür argumentiert werden, dass V2-Stellung in deutschen Nebensätzen nicht durch Assertivität, sondern vielmehr durch das diskurssemantische Konzept At-issueness lizenziert wird.

Wir haben in Abschnitt 2.2 gesehen, dass nur einige Nebensatztypen in der Lage sind, ohne ihren Bezugssatz [+at-issue]-Inhalt auszudrücken, diese Nebensatztypen wurden in Gruppe 1 zusammengefasst (vgl. Tabelle 2.1 in Abschnitt 2.2.5). Auffällig ist nun, dass es sich um dieselben Nebensatztypen handelt, welche in bestimmten Kontexten auch mit V2-Stellung gebildet werden können, wohingegen die Nebensatztypen aus Gruppe 1, die nie ohne ihren Bezugssatz Teil der Hauptstruktur sein können, nur mit VL-Stellung wohlgeformt sind. Diese Übereinstimmung zwischen dem Auftreten von V2-Stellung und der Möglichkeit, [+at-issue]-Inhalt auszudrücken, zeigt sich in Tabelle 7.1 .

Aufgrund dieser Korrelation soll in diesem Abschnitt für die folgende Hypothese argumentiert werden:

\section{At-issueness-Hypothese}

Nur abhängige Sätze, die ohne ihren Bezugssatz [+at-issue]-Inhalt ausdrücken, können V2-Stellung aufweisen. V2-Stellung in abhängigen Sätzen ist eine optionale Markierung für At-issueness.

Kurz: V2 $\rightarrow$ [+at-issue]

Wenn wir davon ausgehen, dass abhängige Sätze durch die drei binären Merkmale [+/-assertives Potential], [+/-at-issue] und [+/-präsupponiert] charakterisiert 


\begin{tabular}{|l|c|c|}
\hline Nebensatztyp & [at-issue] & V2 \\
\hline \hline Komplementsatz, Brückenverb & $+/-$ & $+/-$ \\
\hline Semifaktiver Komplementsatz & $+/-$ & $+/-$ \\
\hline Faktiver Komplementsatz & - & - \\
\hline Temporaler Adverbialsatz & - & - \\
\hline Adverbialsatz mit weil & $+/-$ & $+/-$ \\
\hline Appositiver Relativsatz & - & - \\
\hline Restriktiver Relativsatz, definites Antezedens & - \\
\hline $\begin{array}{l}\text { Restriktiver Relativsatz, indefinites Antezedens, } \\
\text { nicht-spezifisch }\end{array}$ & - & - \\
\hline $\begin{array}{l}\text { Restriktiver Relativsatz, indefinites Antezedens, } \\
\text { spezifisch }\end{array}$ & $+/-$ & $+/-$ \\
\hline
\end{tabular}

Tabelle 7.1.: At-issueness und abhängige V2-Stellung

werden können, ergeben sich insgesamt acht mögliche Merkmalskombinationen, die in Tabelle 7.2 dargestellt sind.

\begin{tabular}{|l||c|c|c|}
\hline Fall & [assertiv] & [at-issue] & [präsupponiert] \\
\hline \hline 1 & + & + & + \\
\hline 2 & + & + & - \\
\hline 3 & + & - & + \\
\hline 4 & + & - & - \\
\hline 5 & - & + & + \\
\hline 6 & - & + & - \\
\hline 7 & - & - & + \\
\hline 8 & - & - & - \\
\hline
\end{tabular}

Tabelle 7.2.: Merkmalsverteilung bei Nebensätzen

Nebensätze mit den Merkmalskombinationen 1, 3 oder 5 treten in der natürlichen Sprache nicht auf, da ein präsupponierter Ausdruck nicht gleichzeitig assertiert oder [+at-issue] sein kann (vgl. dazu die Diskussion in Kapitel 4).

Ein Beispiel für Fall 2 liegt im Deutschen mit weil-V2-Sätzen vor, die ein eigenes assertives Potential besitzen und auch in der Lage sind, [+at-issue]-Inhalt auszudrücken. Da in diesem Fall die Ausprägung des Merkmals [+/-at-issue] mit der des Merkmals [+/-assertives Potential] übereinstimmt, können weil-V2-Sätze jedoch nicht für die Entscheidung darüber, ob die Assertions- oder die At-issuenessHypothese die Lizenzierung von V2-Stellung in abhängigen Sätzen korrekt erfasst, herangezogen werden. Dasselbe gilt für die Fälle 7 und 8, in denen die Merkmale [+/-at-issue] und [+/-assertives Potential] ebenfalls übereinstimmen. Beispiele für Fall 7 bilden Temporalsätze, aber auch faktive Komplementsätze, da beide Nebensatztypen präsupponiert sind (vgl. die Diskussion in Abschnitt 4.2) und folglich 


\section{Lizenzierungsbedingungen}

weder zum Ausdruck von [+at-issue]-Inhalt geeignet sind noch über ein eigenes assertives Potential verfügen. Da die Sätze die beiden Merkmale [-at-issue] und [-assertives Potential] aufweisen, kann auf dieser Grundlage nicht entschieden werden, ob V2-Stellung aufgrund des mangelnden assertiven Potentials ausgeschlossen ist, wie die Assertionshypothese voraussagen würde, oder aber ob die Nicht-Atissueness des Satzes V2-Stellung blockiert. Interessanter ist dagegen Fall 8, welcher im Deutschen mit appositiven Relativsätzen eine Realisierung besitzt. Aufgrund der Tatsache, dass appositive Relativsätze immer [-präsupponiert] sind und dennoch keine V2-Stellung zulassen, konnte in Abschnitt 7.1.2 zumindest ausgeschlossen werden, dass es sich bei [-präsupponiert] um das entscheidende Lizenzierungsmerkmal für das Auftreten von V2-Stellung in abhängigen Sätzen des Deutschen handelt.

Um zu überprüfen, ob die At-issueness- oder aber die Assertionshypothese zutreffend ist, sind die Fälle interessant, in denen die Ausprägung des Merkmals [+/-at-issue] mit dem Merkmal [+/-assertives Potential] nicht übereinstimmt, d.h. die Fälle 4 und 6. Beginnen wir mit einer genaueren Betrachtung von Fall 4. Holler (2008, 202) beobachtet, dass Assertivität nicht auf V2-Sätze beschränkt ist. Sie weist darauf hin, dass auch freie dass-Sätze Ähnlichkeiten mit Assertionen aufweisen. Genau wie weil-V2-Sätze oder V2-Relativsätze kann auch ein freier dass-Satz nicht in einen Interrogativsatz integriert werden, wie das folgende Beispiel nach Holler (2008, 202) belegt. Hier folgt der VL-Satz auf einen Interrogativsatz und sein Inhalt ist nicht Teil der Frageäußerung, sondern drückt vielmehr eine Art sprechaktbezogene Begründung aus.

(263) Ist Max größenwahnsinnig, dass er jetzt noch einen Porsche kauft?

Nach Holler (2008, 202) können freie dass-Sätze zudem Modalpartikeln und andere illokutionäre Indikatoren enthalten, was sie anhand des folgenden Beispiels zeigt:

Max ist größenwahnsinnig, dass er (sicher/wohl/hiermit) noch einen Porsche kauft.

Auch informationsstrukturell verhalten sich freie dass-Sätze wie eigenständige Illokutionen, da eine Integration in die FHG des Matrixsatzes in einer nicht-wohlgeformten Struktur resultiert.

(265) Was ist passiert?

?[Max ist GRÖßenwahnsinnig, dass er jetzt noch einen Porsche kauft.] $]_{F}$

Schließlich lässt sich auch feststellen, dass sich ein Sprecher nicht direkt vom Inhalt eines geäußerten freien dass-Satzes distanzieren kann.

(266) ?Max ist größenwahnsinnig, dass er jetzt noch einen Porsche gekauft hat. Aber ich glaube nicht, dass er das wirklich getan hat.

Freie dass-Sätze verhalten sich in allen vier untersuchten Punkten wie weil-V2-Sätze und somit auch wie Assertionen. Entscheidend ist nun, dass freie dass-Sätze zwar 
assertiv sind, aber nicht für den Ausdruck von [+at-issue]-Inhalt verwendet werden können, wie das folgende Beispiel belegt: ${ }^{.1}$

Q: Wo ist Maria?/Was macht Maria?

B: \#Sie ist verrückt, dass sie bei Regen schwimmen geht.

Freie dass-Sätze weisen somit die Merkmalskombination von Fall 4 auf. Nach der Assertionshypothese müssten sie, da sie assertiv sind, unter Wegfall der Subjunktion auch mit V2-Stellung gebildet werden können. Dies ist jedoch nicht möglich. Beispiel (268-a) ist zwar wohlgeformt, hier handelt es sich aber um zwei juxtapositionierte Hauptsätze. Wird der nachgestellte V2-Satz dagegen intonatorisch in seinen Bezugssatz integriert, ist das Beispiel ungrammatisch (vgl. (268-b)).

(268) a. Max ist größenwahnsinnig, er kauft jetzt noch einen Porsche.

b. *Max ist größenwahnsinnig, (/) er kauft jetzt noch einen Porsche.

Dass freie dass-Sätze, die als Instanz für Fall 4 gelten können, keine V2-Stellung zulassen, zeigt, dass V2-Stellung nicht durch das Merkmal [+assertives Potential] lizenziert wird. Vielmehr untermauert das Verhalten von freien dass-Sätzen die Atissueness-Hypothese.

Die Untersuchung von Fall 6 führt zu einem ganz ähnlichen Ergebnis. Im vorherigen Abschnitt haben wir gesehen, dass Komplement- und Relativsätze mit V2-Stellung über kein eigenständiges Assertionspotential verfügen, was sich u.a. daran zeigt, dass sie informationsstrukturell und intonatorisch in ihren Bezugssatz integriert sind. V2-Komplementsätze können sogar in einen Interrogativsatz integriert werden, was nicht möglich wäre, würde mit der Äußerung eines V2-Komplementsatzes ein eigener assertiver Sprechakt realisiert werden (vgl. Beispiel (238)). Aus diesem Grund führen Gärtner (2001) und Truckenbrodt (2006) zur Rettung der Assertionshypothese das schwächere Konzept der Proto-Assertivität ein. Eine graduelle Aufweichung des ansonsten binären Konzepts der Assertivität ist jedoch nicht notwendig, wenn wir die Idee, derzufolge V2-Stellung mit Assertivität korreliert, aufgeben und stattdessen die At-issueness-Hypothese adaptieren. Von den fünf untersuchten Komplementsatztypen können nur die Sätze, die auch in der Lage sind, ohne ihren Bezugssatz [+at-issue]-Inhalt auszudrücken, im Deutschen mit V2-Stellung gebildet werden. Dazu zählen die Komplemente von Brücken- und semifaktiven Verben, außerdem haben wir gesehen, dass restriktive Relativsätze mit einem indefiniten Antezedens beide Eigenschaften aufweisen. Auch dies zeigt, dass das entscheidende Lizenzierungsmerkmal für V2-Stellung [+at-issue] und nicht [+assertiv] ist.

Weist ein Komplement- oder Relativsatz V2-Stellung auf, wird mit dem abhängigen Satz kein eigenständiger assertiver Sprechakt realisiert. Vielmehr erfährt der in dem Nebensatz ausgedrückte Inhalt eine informationsstrukturelle Aufwertung - entspre-

\footnotetext{
${ }^{51}$ Einige Sprecher des Deutschen scheinen Äußerungen wie (267), bei denen ein freier dass-Satz ohne seinen Bezugssatz die QUD beantwortet, zu akzeptieren. Um diesbezüglich ein abschließendes Urteil treffen zu können, beispielsweise ob es sich dabei um ein dialektales Phänomen handelt, sind sicher empirische Studien notwendig.
} 


\section{Lizenzierungsbedingungen}

chend wurde in der Forschung zu V2-Komplementsätzen auch schon häufig beobachtet, dass der abhängige Satz den inhaltlich relevanten Kern der Gesamtäußerung enthält (vgl. zuletzt Freywald 2013). Durch die V2-Stellung wird angezeigt, dass der Nebensatzinhalt relevant bezogen auf die aktuelle Q ist. Dass V2-Nebensätze [+at-issue]-Inhalt ausdrücken können, zeigen die folgenden Beispiele, in denen der Hauptsatz [-at-issue] ist und der Nebensatz die bezogen auf Q relevante Information ausdrückt.

Q: Wo ist Homer?

B: Marche glaubt, er ist zum Kwik-E-Markt gegangen.

Q: Können eigentlich alle Linguisten lesen?

B: Ich kenne einen Linguisten, der kann nicht lesen.
Kontext: B fängt plötzlich an zu lachen.
Q: Was ist denn los?
B: Ach, es war gerade so witzig, weil Peter ist vom Stuhl gefallen.

Als Beleg für das Zutreffen der At-issueness-Hypothese ist die Beobachtung, dass V2-Nebensätze in der Lage sind, [+at-issue]-Inhalt auszudrücken, jedoch nicht ausreichend. Um ableiten zu können, dass V2-Stellung in den entsprechenden Beispielen durch das Merkmal [+at-issue] lizenziert wird, muss vielmehr gezeigt werden, dass die Nebensätze nur dann V2-Stellung aufweisen können, wenn sie tatsächlich relevanten Inhalt bezogen auf Q ausdrücken. Benötigt wird also ein Kontext, der mit der Merkmalskombination [+assertiv], [-at-issue] und [-präsupponiert] Fall 4 entspricht und für den mit freien dass-Sätzen bereits eine Realisierungsform vorliegt. Die Vorhersage wäre nun, dass sich die Nebensätze aus Gruppe 1, zu der u.a. weilSätze und Komplemente von Brückenverben zählen, in diesem Kontext genau wie freie dass-Sätze verhalten und nur mit VL-Stellung wohlgeformt sind. Im Vergleich zu freien dass-Sätzen ist dies aber mit den Nebensätzen aus Gruppe 1 viel schwieriger zu testen. Wird ein Kontext so manipuliert, dass ein weil-Satz beispielsweise nicht relevant bezogen auf die gegebene Q ist, ist V2-Stellung entgegen der Vorhersage wohlgeformt, wie das folgende Beispiel belegt:

Q: Hat Peter die Klausur bestanden?

B: Er ist leider durchgefallen, weil er hat zu wenig gelernt.

In dem vorliegenden Beispiel wird Q durch den Hauptsatz beantwortet und der Kausalsatz liefert eine zusätzliche Information. Dass der Kausalsatz dennoch V2-Stellung aufweisen kann, ist jedoch noch kein Grund, die At-issueness-Hypothese zu verwerfen. So können wir nicht ausschließen, dass der weil-Satz dazu dient, eine implizite Frage Q' wie beispielsweise Warum ist Peter durchgefallen? zu beantworten, welche sich nach der Klärung von Q stellt. In diesem Fall würde der Kausalsatz bezogen auf Q' dann [+at-issue]-Inhalt ausdrücken und gemäß der At-issueness-Hypothese auch V2-Stellung lizenzieren.

Wird ein V2-Nebensatz als Antwort auf eine Frage Q verwendet, in Bezug auf die er 
eindeutig nicht relevant ist, ist die Äußerung dennoch wohlgeformt. Daraus können wir schließen, dass ein V2-Nebensatz auch in einem Kontext wie (272) als [+at-issue] interpretiert wird. Wir haben bereits in Abschnitt 2.2 gesehen, dass die Nebensatztypen aus Gruppe 1 ambig sind, da sie entweder [+at-issue]- oder [-at-issue]-Inhalt ausdrücken können. Weist der Nebensatz nun V2-Stellung auf, führt das dazu, dass der entsprechende Satz nur noch als [+at-issue] interpretiert werden kann. In einem Kontext wie (272) ist der Nebensatzinhalt bezogen auf die aktuell gegebene Q zwar nicht relevant. Q ist zum Zeitpunkt, zu dem der Nebensatz geäußert wird, jedoch bereits durch den Hauptsatz beantwortet, so dass es den Diskursteilnehmern erlaubt ist, eine neue Frage Q' aufzuwerfen (vgl. dazu Abschnitt 2.1). Die Tatsache, dass der Nebensatz V2-Stellung aufweist, signalisiert H, dass eine implizite Frage Q' rekonstruiert werden muss, auf deren Beantwortung der abhängige Satz abzielt. Die Vorhersage, dass ein V2-Nebensatz in einem [-at-issue]-Kontext nicht wohlgeformt ist, kann folglich nur schwer überprüft werden, da eine [+at-issue]-Lesart unter Rekonstruktion einer impliziten Q' erzwungen wird, sobald ein Nebensatz aus Gruppe 1 V2-Stellung aufweist. Die Nebensätze aus Gruppe 2 erlauben dagegen keine solche Uminterpretation, da sie konventionell als Träger von [-at-issue]-Informationen markiert sind.

Wir haben bereits in Abschnitt 2.1.3 gesehen, dass natürliche Sprachen unterschiedliche Mittel bereithalten, um Bedeutungsaspekte als plus oder minus at-issue zu markieren. Beispielsweise sind Träger von Fokusmerkmalen immer [+at-issue], wohingegen der definite Artikel markiert, dass die Existenz der entsprechenden Entität nicht zur Debatte steht. Die sprachliche Markierung von [+/-At-issueness] ist insbesondere dann wichtig, wenn die kontextuell relevante Q implizit ist und demnach nur anhand solcher sprachlicher Markierungen geschlossen werden kann, welche Frage mit der jeweiligen Äußerung beantwortet werden soll. Bereits in Abschnitt 2.1.3 wurde festgestellt, dass ein und derselbe Satz dazu dienen kann, die unterschiedlichsten Fragen zu beantworten. Beispielsweise bildet die Äußerung in (273) eine wohlgeformte Antwort auf die beiden in (274) dargestellten Fragen sowie auf zahlreiche weitere. Als Antwort auf (274-a) ist lediglich der Hauptsatzinhalt relevant, während in einem Kontext wie (274-b) der eingebettete Satz die [+at-issue]-Information ausdrückt.

(273) Homer glaubt, dass Marche heute länger arbeiten muss.

(274) a. Wer glaubt, dass Marche heute länger arbeiten muss?

b. Wo ist Marche?

Aus einer diskurssemantischen Perspektive betrachtet ist ein komplexes Satzgefüge bestehend aus einem Haupt- und einem Nebensatz immer dann ambig, wenn es sich um einen Nebensatztyp aus Gruppe 1 handelt, der potentiell in der Lage ist, [+atissue]-Inhalt auszudrücken. Weist der abhängige Satz nun V2-Stellung auf wie in Beispiel (275), wird angezeigt, dass der Neben- und nicht der Hauptsatz die bezogen auf Q relevante Information ausdrückt. Die folgende Äußerung kann nun nicht mehr als Antwort auf Frage (274-a) verwendet werden, wohl aber in einem Kontext wie 
(274-b).

Homer glaubt, Marche muss heute länger arbeiten.

Natürlich kann eine Äußerung wie (275) auch eine andere Frage als (274-b) beantworten, beispielsweise die Frage Q'=Warum ist Marche so schlecht gelaunt?. Wir haben bereits in Abschnitt 2.1.3 gesehen, dass die jeweils relevante Q auch durch sprachliche Markierungen nicht eindeutig bestimmt wird, sondern unterspezifiziert bleibt. Entscheidend ist jedoch, dass andere mögliche Fragen wie (274-a) durch die At-issuess-Markierung ausgeschlossen werden.

Auch ein komplexes Satzgefüge bestehend aus einem weil- und seinem Bezugssatz weil $(\mathrm{p}, \mathrm{q})$ ist ambig bezogen darauf, welche $\mathrm{Q}$ beantwortet werden soll. Weist der Kausalsatz p nun V2-Stellung auf, kann die Äußerung auf keinen Fall eine Frage nach dem Zutreffen von q (also q?) beantworten, wohl aber die Frage p?. V2-Stellung ist in diesem Fall folglich mit einer Selektionsbeschränkung für Kontexte verbunden (vgl. dazu Onea 2011): Eine Äußerung des Typs weil(p,q) kann nur dann in einem Kontext $\mathrm{Q}=\mathrm{q}$ ? verwendet werden, wenn $\mathrm{p}$ mit VL-Stellung realisiert wird.

V2-Stellung in abhängigen Strukturen zeigt an, dass der entsprechende Nebensatz [+at-issue]-Inhalt ausdrückt und gibt somit einen Hinweis darauf, wie die Q lautet, auf deren Beantwortung S abzielt. Da die Nebensatztypen aus Gruppe 1 aber auch mit VL-Stellung die bezogen auf Q relevante Information ausdrücken können (vgl. die Beispiele in Abschnitt 2.2), können wir schließen, dass es sich nur um eine optionale Markierung für At-issueness handelt. Möglicherweise ist die Verwendung eines VL-Nebensatzes aber mit einer Implikatur verbunden, im Zuge derer H schließt, dass der Nebensatz [-at-issue] ist. Handelt es sich um einen Nebensatztyp aus Gruppe 1, kann S zwischen zwei alternativen Formen wählen, und zwar einem bezüglich seines Diskursstatus ambigen VL-Satz und einem nicht-ambigen V2-Satz. Wird nun ein VL-Satz geäußert, wird dieser möglicherweise präferiert als [-at-issue] interpretiert, da S, sollte der Nebensatz relevant bezogen auf Q sein, auch eine nicht-ambige Alternative hätte wählen können. Ob dieser pragmatische Blocking-Effekt tatsächlich besteht, bedarf allerdings noch einer empirischen Überprüfung.

In den Kontexten, in denen V2-Stellung ausgeschlossen ist, liegt immer eine [-atissue]-Lesart vor. Dies trifft auf alle Nebensätze aus Gruppe $2 \mathrm{zu}$, die grundsätzlich nicht zur Beantwortung der jeweils relevanten Q geeignet sind und dementsprechend auch nie V2-Stellung aufweisen können.

Der große Vorteil der in diesem Abschnitt vorgestellten Analyse gegenüber der Assertionshypothese besteht darin, dass das Auftreten bzw. Fehlen von V2-Stellung in abhängigen Sätzen mithilfe der At-issueness-Hypothese auch in Kontexten erklärt werden kann, die durch die Assertionshypothese nur unzureichend erfasst werden. Dass bestimmte Nebensätze Ähnlichkeiten zu Assertionen aufweisen, obwohl sie illokutionär abhängig sind, kann dann darauf zurückgeführt werden, dass der propositionale Inhalt des Satzes informationsstrukturell aufgewertet werden soll. So wird in dem folgenden Beispiel durch die V2-Stellung im Nebensatz markiert, dass die abhängige Proposition relevant bezogen auf $\mathrm{Q}$ ist, es handelt sich aber um keine 
Sprecherassertion, auf deren Inhalt sich $\mathrm{S}$ festlegt.

Q: Wer hat das Armband gestohlen?

B: Peter glaubt, es war Maria.

Attraktiv an der At-issueness-Hypothese ist zudem, dass mit einem Konzept gearbeitet wird, welches bereits unabhängig von der Beschäftigung mit Wurzelphänomenen motiviert ist und mithilfe von klar definierten Tests ermittelt werden kann (vgl. dazu Abschnitt 3). Dass präsupponierte Nebensätze nicht mit V2-Stellung gebildet werden können, ergibt sich ebenfalls direkt aus der At-issueness-Hypothese, da präsupponierte Inferenzen als eine Untermenge der [-at-issue]-Inhalte analysiert wurden (vgl. Abbildung 4.1 in Kapitel 4.1.4). ${ }^{52}$

Natürlich decken sich assertierte und [+at-issue]-Inhalte größtenteils, was damit zusammenhängt, dass die Form eines Ausdrucks zu dessen diskurssemantischer Funktion passen sollte. Informationen, welche besonders zentral sind, da sie bezogen auf die aktuelle Q relevant sind, werden typischerweise auch assertiert. Nichtsdestotrotz handelt es sich bei At-issueness um ein Konzept, welches sich primär auf den propositionalen Inhalt und dessen diskurssemantische Relevanz bezieht, wohingegen Assertivität auf eine spezielle Form des Sprechakts verweist. Um die beiden Konzepte Assertivität und At-issueness voneinander abzugrenzen, wurden unterschiedliche Tests verwendet. [+At-issue]-Inferenzen können immer nur in Relation zu einer kontextuell gegebenen $\mathrm{Q}$ ermittelt werden, zudem projizieren sie nicht und sind nicht sensibel für einen direkten Widerspruch durch H. Um Assertionen zu bestimmen, wurde dagegen überprüft, ob eine Äußerung in einen Interrogativsatz sowie in die Matrix-FHG integriert werden kann, ob sie Modalpartikeln enthalten kann und ob sie sensibel für eine Distanzierung durch $\mathrm{S}$ ist. ${ }^{53}$ Auf diese Weise konnte gezeigt werden, dass At-issueness und Assertivität nicht immer deckungsgleich sind. So weisen freie dass-Sätze zwar Ähnlichkeiten zu Assertionen auf, sie können aber nie zum Ausdruck von [+at-issue]-Inhalt verwendet werden. Bei V2-Komplement- und Relativsätzen ist es genau umgekehrt, da diese Sätze zwar [+at-issue] sind, sich aber

${ }^{52}$ Wie in Abschnitt 6.4 angedeutet, können auch die Nebensätze aus Gruppe 1 nicht in jedem Kontext V2-Stellung aufweisen - V2-Stellung ist beispielsweise blockiert, wenn der abhängige Satz topikalisiert ist, wie die folgenden Beispiele belegen (vgl. dazu auch Antomo/Steinbach 2010, Breindl 2009, Gärtner 2001, Holler 2008 und Uhmann 1998):

(i) a. *Weil es hat geregnet, ist die Straße ganz nass.

b. *Einen Mann, der kann nicht lesen, kenne ich.

Möglicherweise lassen sich einige dieser Auftretensbeschränkungen ebenfalls mithilfe der Atissueness-Hypothese ableiten. So wurde in Fußnote 15 in Abschnitt 2.2.2 bereits darauf hingewiesen, dass topikalisierte Nebensätze nicht dazu geeignet sind, ohne ihren Bezugssatz [+atissue]-Inhalt auszudrücken.

${ }^{53}$ Bentzen (2009) und Wiklund et al. (2009) verwenden zum Teil andere Strategien, um Assertionen von [+at-issue]-Inhalten zu unterscheiden. So gehen sie davon aus, dass nur assertierte Inhalte durch einen direkten Widerspruch durch $\mathrm{H}$ angefochten werden können. Dass auf diese Weise jedoch nicht, wie traditionell angenommen, Assertionen von Präsuppositionen, sondern [+atissue]- von [-at-issue]-Inferenzen abgegrenzt werden, wurde bereits in Abschnitt 3.2 gezeigt. 


\section{Lizenzierungsbedingungen}

nicht wie Assertionen verhalten.

Die At-issueness-Hypothese besagt, dass ein abhängiger V2-Satz immer [+at-issue]Inhalt ausdrückt, der umgekehrte Schluss, demzufolge [+at-issue]-Inhalte nur in V2Sätzen transportiert werden können, trifft dagegen nicht zu, da V2-Stellung immer optional ist. Dies muss bei einer syntaktischen Implementierung der At-issuenessHypothese bedacht werden, auf deren genaue Ausarbeitung im Rahmen dieser Arbeit allerdings verzichtet wird. Analog zur Analyse von Haegeman (2003), Haegeman (2006) und Julien (2007), die den Unterschied zwischen Nebensätzen mit und ohne Wurzelphänomenen auf deren interne Syntax zurückführen, wobei Nebensätze mit Wurzelphänomenen eine komplexe linke Peripherie inklusive einer ForceP besitzen, wohingegen Nebensätze ohne Wurzelphänomene nur eine reduzierte C-Domäne aufweisen, ist jedoch auch grundsätzlich eine strukturelle Repräsentation von Atissueness im Rahmen einer Split-C-Theorie denkbar. Die Existenz eines syntaktischen At-issueness-Kopfes sollte dann zumindest in einigen Sprachen einen morphosyntaktischen Reflex besitzen, beispielsweise argumentiert Schardl (2012), dass es sich bei der finnischen Partikel - $h A n$ um eine morphologische Markierung für Atissueness handelt.

Ob V2-Nebensätze eine andere interne Syntax, d.h. eine reichere linke Peripherie mit einer ForceP oder aber alternativ einem At-issueness-Operator, besitzen als kanonische VL-Nebensätze muss hier offen bleiben. Gut untersucht ist dagegen die externe Syntax von V2-Nebensätzen, wobei in der Forschung diesbezüglich Konsens darüber besteht, dass sich V2-Nebensätze durch einen höheren Grad an Desintegration als ihre kanonischen VL-Gegenstücke ausweisen (für Details zu den einzelnen V2-Nebensatztypen vgl. Abschnitt 6.4).

Bei abhängigen V2-Strukturen handelt es sich um ein großes Phänomen, das neben den hier untersuchten Nebensatztypen noch zahlreiche weitere Sätze betrifft, sei es, weil sie V2-Stellung aufweisen können oder gerade nicht. Zudem existieren abhängige V2-Sätze auch in anderen germanischen Sprachen, wobei die Lizenzierungskontexte interessanterweise sehr ähnlich sind. Deswegen ist es sinnvoll, zu überprüfen, ob die At-issueness-Hypothese auch korrekte Vorhersagen bezogen auf diese Strukturen macht. Neben V2-Stellung existieren im Deutschen außerdem zahlreiche weitere Desintegrationsphänomene, die dazu führen, dass sich auch VL-Sätze nicht wie kanonisch integrierte Nebensätze verhalten. Ob auch diese Phänomene mit At-issueness korrelieren, ist bislang noch offen. Beide Aspekte sollen im Folgenden an ausgewählten Beispielen kurz diskutiert werden, zudem wird am Beispiel der eingebetteten Topikalisierung im Englischen untersucht, ob die At-issueness-Hypothese auch auf dieses Wurzelphänomen angewandt werden kann. 


\subsection{Weitere Anwendungsbereiche der At-issueness-Hypothese}

\subsubsection{Andere abhängige V2-Strukturen}

Die At-issueness-Hypothese sollte idealerweise nicht nur das Auftreten von V2Stellung in den hier untersuchten acht Nebensatztypen erfassen, sondern darüber hinaus auch (i) auf weitere V2-Nebensätze bzw. V2-Blockierer im Deutschen anwendbar sein und (ii) die Distribution von V2-Stellung in abhängigen Sätzen in anderen germanischen Sprachen berücksichtigen. Beide Aspekte werden im Folgenden kurz angeschnitten, jedoch nicht abschließend geklärt. Bezogen auf die Frage, ob die At-issueness-Hypothese auch das Auftreten von V2-Stellung in anderen als den bislang untersuchten acht Nebensatztypen erfasst, werden im folgenden kurz obwohl-V2-Sätze, Konditional- und $d a-$ Sätze diskutiert.

Dass obwohl in der gesprochenen Sprache auch dafür verwendet wird, einen V2Satz einzuleiten, ist von der Forschung gut dokumentiert. Zwischen kanonischen obwohl-VL-Sätzen und obowhl-V2-Sätzen existieren zentrale formale und funktionale Unterschiede, die ausführlich von u.a. Antomo/Steinbach (2013), Gaumann (1983), Günthner (1999b) und Pittner (1999, 268ff) diskutiert und hier nur verkürzt dargestellt werden. Die Form betreffend wurde schon häufig beobachtet, dass ein obwohl-V2-Satz wie (277-b) anders als sein kanonisches VL-Gegenstück in (277-a) syntaktisch und prosodisch selbstständig ist, zudem folgt auf obwohl eine intonatorische Pause.

a. Ich kaufe diesen Rock, obwohl er ein Loch hat.

b. Ich kaufe diesen Rock. Obwohl: Er hat ein Loch.

Zudem bilden obwohl-V2-Sätze einen eigenen Sprechakt, wie u.a. Günthner (1999b), Pasch et al. (2003, 369ff) und Pittner (1999, 269) beobachten, wohingegen kanonisch integrierte obwohl-VL-Sätze nach König (1991) immer präsupponiert und Teil der Matrixillokution sind. Was ihre Bedeutung betrifft, besteht ebenfalls ein zentraler Unterschied zwischen den zwei Arten von obwohl-Sätzen: Mit der Äußerung von Beispiel (277-a) assertiert S, dass sie den Rock trotz des Mangels kaufen will, und somit sowohl p als auch q wahr sind. Dagegen führt die Äußerung (277-b) zu einer Infragestellung des zuvor geäußerten Kaufvorhabens, was Antomo/Steinbach (2013) in einer Pilotstudie empirisch belegen konnten.

Wie weil-V2-Sätze sind auch obwohl-Sätze mit V2-Stellung nicht eingebettet, sondern selbstständige Hauptsätze. Da ein mit obwohl eingeleiteter Satz aber nur in bestimmten Kontexten V2-Stellung aufweisen kann, stellt sich dennoch (genau wie bei weil-V2-Sätzen, vgl. dazu Abschnitt 6.2) die Frage, welche besondere Eigenschaft diese Kontexte besitzen müssen, um einen V2-Satz zu lizenzieren. Ausgehend von der At-issueness-Hypothese ist zu erwarten, dass ein V2-Satz, der auf obwohl folgt, immer [+at-issue]-Inhalt ausdrückt. Aber wie könnte eine Frage lauten, die durch einen obwohl-V2-Satz wie in Beispiel (277-b) beantwortet wird? Es handelt sich um 


\section{Lizenzierungsbedingungen}

die gleiche QUD, auf deren Beantwortung bereits der vorangestellte Bezugssatz abzielt. Nehmen wir einmal an, dass der erste Satz aus Beispiel (277-b), also Ich kaufe diesen Rock, die Frage $\mathrm{Q}=$ Wirst du diesen Rock kaufen? beantwortet. In diesem Fall ist die entsprechende Q zum Äußerungszeitpunkt des nachgestellten obwohlV2-Satzes bereits vollständig beantwortet. Der obwohl-V2-Satz revidiert die zuvor getätigte Antwort jedoch und zeigt an, dass die entsprechende Q durch den Bezugssatz doch noch nicht abschließend geklärt wurde, sondern nach wie vor offen ist. Dabei liefert der obwohl-Satz keine komplette Antwort auf Q, vielmehr wird durch den Konzessivsatz eine SubQ, nämlich in diesem Fall In welchem Zustand ist der Rock? beantwortet, woraus auf eine Antwort auf Q pragmatisch geschlossen werden kann (aber nicht muss): Normalerweise kauft man keinen Rock, der ein Loch hat, also werde ich den Rock nicht kaufen. ${ }^{54}$

Wie weil-V2- können auch obwohl-V2-Sätze auf der epistemischen oder der sprechaktbezogenen Ebene interpretiert werden (für eine genauere Beschreibung der unterschiedlichen Lesarten von obwohl-Sätzen vgl. Antomo/Steinbach 2013, Günthner 1999b und Pittner 1999). Bei der epistemischen Lesart besteht der konzessive Kontrast nicht zwischen den beiden Propositionen, sondern zwischen der Bezugssatzproposition und dem Wissenszustand von S. So wird mit Beispiel (278) ausgedrückt, dass S, da er selbst noch nicht in Südamerika war, eigentlich nichts über die Attraktivität dieser Region aussagen kann.

(278) Südamerika ist toll. Obwohl: Da gewesen bin ich selbst noch nicht.

In diesem Fall zielt der obwohl-V2-Satz auf die Beantwortung der Frage Warst du schon einmal in Südamerika? ab. Dabei handelt es ich um eine Unterfrage der übergeordneten QUD, die in etwa Wie ist es in Südamerika? oder Wie findest du Südamerika? lautet. Indem die SubQ im nachgestellten obwohl-Satz verneint wird, drückt $\mathrm{S}$ aus, dass sie eigentlich nicht in der Lage ist, die QUD zu beantworten, wodurch die im vorangestellen Bezugssatz enthaltene komplette Antwort auf die QUD revidiert bzw. abgeschwächt wird.

Obwohl-V2-Sätze können nicht nur epistemisch interpretiert werden, sondern auch eine sprechaktbezogene Modifikation ausdrücken. Wie Bach $(1999,342)$ und Scheffler (2008, 135) zeigen, besteht der konzessive Kontrast in Beispiel (279) zwischen der nachgestellten Proposition p und der Tatsache, dass q geäußert wurde.

Mein Klient hat ein Alibi. Obwohl: Eigentlich hat der Richter verboten, darüber zu sprechen.

\footnotetext{
${ }^{54}$ Günthner (1999b) geht davon aus, dass obwohl-V2-Sätze zu einer Rücknahme des zuvor Gesagten führen. In einer empirischen Pilotstudie zeigen Antomo/Steinbach (2013) jedoch, dass dieser Begriff zu stark ist, entsprechende Beispiele wurden nur in 4,6 Prozent aller Fälle als Zurücknahme interpretiert. Sicher ist jedoch, dass die Bezugssatzproposition q infrage gestellt wird, ohne weiteren Kontext kann jedoch nicht darüber entschieden werden, ob S bezogen auf Beispiel (277-b) den Rock nun kaufen wird oder nicht.
} 
Auch in diesem Fall wird durch den vorangestellten Bezugssatz die jeweilige QUD (hier: Ist der Klient schuldig?) komplett beantwortet. Der nachgestellte obwohl-V2Satz zielt nun auf die Beantwortung einer Unterfrage der eigentlich bereits geschlossenen QUD ab, nämlich SubQ = Darfst du dich zu dem Thema äußern?. Durch die Verneinung dieser SubQ und somit der Verneinung einer wesentlichen Voraussetzung zur Beantwortung der QUD wird wie im Falle der epistemischen Lesart die im Bezugssatz geleistete Antwort eingeschränkt (bzw. die daraus entstehenden Konsequenzen, nämlich mehr zum Thema zu sagen, werden nicht gezogen).

Wir können festhalten, dass obwohl-V2-Sätze immer eine SubQ der durch den vorangestellten Bezugssatz beantworteten QUD beantworten - durch die negative Antwort auf die jeweilige SubQ wird die zuvor getätigte komplette Antwort auf die QUD revidiert bzw. infrage gestellt. ${ }^{55}$

Anders als obwohl und weil kann der konditionale Konnektor wenn im Deutschen nur VL-Sätze einleiten. Dies wird durch die At-issueness-Hypothese nicht korrekt vorhergesagt, denn Konditionalsätze können, wie das folgende Beispiel belegt, offensichtlich dafür verwendet werden, [+at-issue]-Inhalt auszudrücken: ${ }^{56}$

Q: Wo ist Maria?

A: Wenn sie in Paris ist, wäre ich echt sauer.

Mit wenn eingeleitete Konditionalsätze können zwar keine V2-Stellung aufweisen, dies gilt jedoch nicht für alle Konditionalsätze. Wie Volodina (2013) zeigt, können konditionale V2-Sätze u.a. wie folgt eingeleitet werden:

Gesetzt den Fall/Angenommen/Vorausgesetzt sie ist in Paris, wäre ich echt sauer.

Möglicherweise liegt im konditionalen Bereich eine lexikalische Ausdifferenzierung vor, wobei wenn immer VL-Sätze einleitet, die entweder [+at-issue] oder [-at-issue] sein können, wohingegen die in Beispiel (281) genannten Satzeinleiter immer V2Sätze einleiten, welche immer [+at-issue] sind. Dies muss von der Forschung allerdings noch genauer untersucht werden.

Eine klare lexikalische Ausdifferenzierung liegt dagegen im kausalen Bereich vor.

\footnotetext{
${ }^{55}$ Auch weil-Sätze mit epistemischer oder sprechaktbezogener Lesart beantworten immer eine eigene Frage. Während der epistemische Kausalsatz in Beispiel (i-a) auf die Frage Woher weißt $d u$ das? abzielt, beantwortet der sprechaktbezogene weil-Satz in (i-b) die Frage Wieso sagst du das?.

(i) a. Es hat geschneit. Weil die Straße ist ganz nass.

b. Brauchst du noch Milch? Weil ich gehe jetzt einkaufen.

${ }^{56}$ Aus dem Konditionalsatzgefüge folgt natürlich nicht, dass Maria in Paris ist, Q wird durch den Satz also nicht beantwortet. Gemessen an der Definition (25) nach Simons et al. (2011), derzufolge eine Proposition p dann at-issue ist, wenn die mit ihr verbundene Entscheidungsfrage ?p relevant für die Klärung von Q ist (vgl. Abschnitt 2.1.2), ist der Konditionalsatz in Beispiel (280) jedoch genauso at-issue wie etwa Komplementsätze von Brückenverben in einem ähnlichen Kontext.
} 


\section{Lizenzierungsbedingungen}

Wir haben bereits gesehen, dass weil in der gesprochenen Sprache entweder einen VL- oder aber einen V2-Satz einleiten kann, wobei in diesem Kapitel dafür argumentiert wurde, dass weil-V2-Sätze immer [+at-issue] sind, wohingegen weil-VL-Sätze auch [-at-issue]-Inhalt ausdrücken können. Der kausale Konnektor $d a$ ist dagegen nur mit VL-Stellung wohlgeformt. Interessanterweise argumentieren AverintsevaKlisch/Volodina (2012) nun dafür, dass $d a$-Sätze nie Teil der Hauptstruktur sind, sondern immer [-at-issue]-Inhalt ausdrücken (für mehr Details vgl. AverintsevaKlisch/Volodina 2012).

Die Übertragung der At-issueness-Hypothese auf andere abhängige Sätze des Deutschen (neben den hier untersuchten acht Nebensatztypen) wurde hier nur kurz angeschnitten und bedarf sicherlich noch weiterer Klärung. Zudem müssten noch weitere, hier nicht untersuchte V2-Lizenzierer (wie beispielsweise Präferenzprädikate) und V2-Blockierer (wie z.B. Modal- oder Finalsätze) daraufhin untersucht werden, ob das Auftreten bzw. Fehlen von V2-Stellung in diesen Kontexten ebenfalls mit +/-At-issueness korreliert. Dabei sollte allerdings nicht ausgeschlossen werden, dass dem Auftreten von V2-Stellung in abhängigen Sätzen möglicherweise auch mehrere Lizenzierungsfaktoren zugrundeliegen oder dass V2-Stellung in bestimmten Kontexten aufgrund von rein strukturellen Gründen ausgeschlossen ist.

Neben dem Deutschen weisen auch andere germanische V2-Sprachen abhängige V2Strukturen auf und interessanterweise lassen sich zahlreiche Parallelen in der Distribtuion nachweisen (für einen Überblick vgl. u.a. Bentzen 2010, Heycock 2005, Iatridou/Kroch 1992, Julien 2007, Vikner 1995 und Weerman 1989). Beispielsweise zeigen de Haan/Weerman (1985), dass im Friesischen genau wie im Deutschen zwar Komplementsätze von Brückenverben V2-Stellung aufweisen können (anders als im Deutschen ohne Wegfall des Komplementierers), nicht aber Nebensätze, die unter dem faktiven Verb bedauern eingebettet sind.

a. Pyt sei dat hy hie my sjoen.

Pyt sagte dass er hat mich gesehen.

b. *Pyt betreuret dat hy hie my sjoen.

Pyt bedauert dass er hat mich gesehen.

Dieselbe Beobachtung trifft auch auf andere germanische Sprachen wie beispielsweise Dänisch, Norwegisch und Schwedisch zu, wo die sogenannten Brückenverben wie im Deutschen einen V2-Satz einbetten können (ohne Wegfall des Komplementierers), faktive Verben dagegen nicht. Ein Desiderat der Forschung zu abhängigen V2Strukturen in germanischen Sprachen besteht darin, die Distribution von abhängiger V2-Stellung in allen Limited Embedded V2-Sprachen uniform über denselben Lizenzierungsfaktor zu erklären. Der prominenteste Ansatz in dieser Richtung ist die weiter oben vorgestellte Assertionshypothese, wobei wir schon gesehen haben, dass deren Anwendung auf das Deutsche mit einigen Problemen verbunden ist. ${ }^{57}$

${ }^{57}$ Dass die Assertionshypothese auch bezogen auf das Auftreten von abhängiger V2-Stellung im Färöischen, Isländischen, Norwegischen und Schwedischen z.T. falsche Vorhersagen macht, zeigen Wiklund et al. (2009). 
Bezogen auf Färöisch, Isländisch, Norwegisch und Schwedisch untersuchen Wiklund et al. (2009), ob ein Zusammenhang zwischen dem Auftreten von V2-Stellung in Komplementsätzen und At-issueness besteht. Sie zeigen, dass in den von ihnen diskutierten Sprachen nur Komplementsätze, die auch ohne ihren Bezugssatz eine Frage beantworten können und demnach den sogenannten Main Point of Utterance (kurz: MPU) bilden, V2-Stellung aufweisen können - damit deckt sich ihr Ergebnis mit den in dieser Arbeit gemachten Beobachtungen bezogen auf Komplementsätze des Deutschen. Allerdings kommen Wiklund et al. (2009) zu dem Schluss, dass At-issueness keine notwendige Voraussetzung für das Auftreten von V2-Stellung in Nebensätzen in den von ihnen untersuchten Sprachen ist. Um zu zeigen, dass es sich bei Atissueness nicht um das entscheidende Lizenzierungsmerkmal handeln kann, führen sie das folgende Beispiel (ihr Beispiel 50) aus dem Schwedischen an:

Q: Varför kom han inte på festen?

'Why didn't he come to the party?'

A: Kristine sa att fick inte.

Kristine said that he got not.

'Kristine said that he wasn't allowed to.'

Die Antwort von A enthält einen V2-Nebensatz (ohne Comp-drop), der unter dem Brückenverb sagen eingebettet ist. Entscheidend ist nun, dass A's Äußerung in diesem Kontext zwei Lesarten haben kann: Entweder beantwortet der Nebensatz alleine die Frage Q, in diesem Fall ist er [+at-issue] und das Auftreten der V2-Stellung konform mit der At-issueness-Hypothese. Die Antwort A kann nach Wiklund et al. (2009) aber auch so interpretiert werden, dass der Gesamtsatz dazu dient, Q zu beantworten, dann ist der Nebensatz nur ein Teil der at-issue-Bedeutung und sollte nach der At-issueness-Hypothese nicht mit V2-Stellung wohlgeformt sein. Dass auch die zuletzt genannte Lesart für Beispiel (283) zugängig ist, werten Wiklund et al. (2009) als Indiz dafür, dass die Regel V2 $\rightarrow$ [+at-issue] für das Schwedische nicht zutreffend ist.

Es ist fraglich, ob Wiklund et al. (2009)'s Kritikpunkt auch auf die Distribution von abhängiger V2-Stellung im Deutschen zutrifft. Wird das entsprechende Beispiel ins Deutsche übersetzt, ist intuitiv nur schwer zu beurteilen, ob A's Äußerung tatsächlich ambig ist.

(284) Q: Warum ist er nicht zur Party gekommen?

A: Kristine hat gesagt, er darf nicht.

Die Lesart, derzufolge der Gesamtsatz at-issue ist, kann wie folgt paraphrasiert werden: Der Grund für sein Fehlen war nicht, dass er nicht durfte, sondern dass Kristine das gesagt hat (wenn beispielsweise Peter ihm verboten hätte, zur Party zu gehen, wäre er hingegangen). In dieser Lesart müsste das Matrixsubjekt Kristine betont sein, dann wäre der Satz jedoch nur mit VL-Stellung wohlgeformt. Wiklund et al. (2009)'s Gegenbeispiel scheint zumindest fragwürdig - wünschenswert wäre hier eine empirische Studie, um zu testen, ob A's Äußerung in (283) bzw. (284) tatsächlich 


\section{Lizenzierungsbedingungen}

die Lesart zulässt, in der der Gesamtsatz at-issue ist.

Bezogen auf die Frage, inwieweit die At-issueness-Hypothese auch die Distribution von V2-Stellung in anderen abhängigen Sätzen des Deutschen und anderen germanischen Limited Embedded V2-Sprachen erklären kann (und ob es überhaupt einen einzigen Lizenzierungsfaktor für all diese Kontexte gibt), besteht noch eine Menge Forschungsbedarf, wobei einige mögliche Forschungsrichtungen hier aufgezeigt wurden.

\subsubsection{Eingebettete Topikalisierung im Englischen}

Auch wenn die Assertionshypothese das Auftreten von V2-Stellung im Deutschen nicht korrekt erfasst, muss sie deswegen nicht generell verworfen werden. Hooper/ Thompson (1973) haben den Gedanken, dass die Distribution von Wurzelphänomenen mit den Konzepten Assertion und Präsupposition zusammenhängt, ursprünglich für das Englische formuliert und wir werden in diesem Abschnitt sehen, dass dies zutreffend ist. Exemplarisch sollen zu diesem Zweck eingebettete Topikalisierungen und Linksversetzungen betrachtet werden. Um die Distribution von eingebetteten Topikalisierungen im Englischen mit der von V2-Stellung in deutschen Nebensätzen vergleichen zu können, werden im Folgenden die wichtigsten Lizenzierungskontexte untersucht. Anhand der Ergebnisse wird anschließend diskutiert, ob die Distribution von eingebetteten Topikalisierungen im Englischen besser durch die Assertions- oder durch die At-issueness-Hypothese erfasst wird.

Beginnen wir mit Nebensätzen, welche die Argumentforderung eines Verbs erfüllen. Genau wie im Deutschen können auch im Englischen Komplementsätze von Brückenverben Wurzelphänomene aufweisen (vgl. u.a. Hooper/Thompson 1973 und Maki et al. 1999). Dies wird durch das folgende Beispiel illustriert, in dem eine Topikalisierung des Nebensatzobjekts vorliegt.

John believes that this book, Mary read.

Auch die Komplemente von semifaktiven Verben erlauben die Topikalisierung eines Arguments (vgl. Beispiel (286-a)) und Linksversetzungen wie in Beispiel (286-b) (beide Beispiele nach Hooper/Thompson 1973, 482).

a. We saw that each part he had examined carefully.

b. I discovered that this book, it has the recipes in it.

Im Gegensatz dazu sind Wurzelphänomene im Englischen genau wie im Deutschen in faktiven Komplementsätzen blockiert:

*John regrets that this book, Mary read.

Auch bei der Betrachtung von Adverbialsätzen können wir feststellen, dass Wurzelphänomene im Englischen in den gleichen Kontexten möglich sind wie im Deutschen. Im Englischen kann eine Phrase linksversetzt werden, wenn sie Teil eines Kausalsatzes ist (vgl. Beispiel (288-a) nach Sawada/Larson 2004). Dagegen erhalten 
wir einen ungrammatischen Satz, wenn eine Linksversetzung in einem Temporalsatz vorliegt wie in Beispiel (288-b).

(288) a. Mildred drives a Mercedes because her son, he owns stock in Xerox.

b. *Mildred bought a Mercedes when/before/after her son, he purchased stock in Xerox.

Wie sieht es nun mit der Distribution von Topikalisierungen in englischen Relativsätzen aus? Restriktive Relativsätze, die von einer definiten Nominalphrase abhängen, können im Englischen nie Voranstellungstransformationen untergehen, wie in Beispiel (289) nach Hooper/Thompson $(1973,489)$ gezeigt:

*The car that only rarely did I drive is in excellent condition.

Bezieht sich ein restriktiver Relativsatz dagegen auf ein indefinites Antezedens wie in Beispiel (290) nach Hooper/Thompson (1973, 490), ist die Voranstellung einer Konstituente möglich:

(290) I saw a dress which under no circumstances would I have bought.

Demnach verhalten sich auch restriktive Relativsätze, was die Lizenzierung von Wurzelphänomenen betrifft, im Deutschen und im Englischen gleich. Anders als im Deutschen können im Englischen aber appositive Relativsätze syntaktische Transformationen, welche sonst auf selbstständige Sätze beschränkt sind, aufweisen. Beispielsweise kann die Adverbphrase only rarely in dem folgenden Beleg von Hooper/Thompson $(1973,488)$ problemlos topikalisiert werden.

This car, which only rarely did I drive, is in excellent condition

Wir können festhalten, dass die Distribution von V2-Stellung in deutschen Nebensätzen und von eingebetteten Voranstellungstransformationen im Englischen gleich ist, was Komplement- und die zwei hier untersuchten Adverbialsatztypen betrifft. Ein Unterschied ergibt sich jedoch bei der Betrachtung von Relativsätzen: Während appositive Relativsätze im Deutschen V2-Stellung blockieren, können sie im Englischen Wurzeltransformationen aufweisen. Die Ergebnisse dieser Gegenüberstellung sind in Tabelle 7.3 dargestellt. ${ }^{58}$

\footnotetext{
${ }^{58}$ Werden noch weitere Adverbialsatztypen in die Gegenüberstellung miteinbezogen, lassen sich auch hier Unterschiede zwischen dem Deutschen und dem Englischen feststellen. Im Englischen können beispielsweise Konditionalsätze in Abhängigkeit ihrer Interpretation Wurzelphänomene aufweisen. Bezieht sich ein Konditionalsatz auf den propositionalen Inhalt seines Bezugssatzes wie in Beispiel (i-a) (nach Haegeman 2006, 1656), führt eine Topikalisierung zu einer ungrammatischen Struktur, wohingegen Konditionalsätze, die die Illokutionsebene modifizieren wie in Beispiel (i-b), Topikalisierungen zulassen (vgl. Haegeman 2006).
}

(i)

a. *If these exams you don't pass, you won't get the degree.

b. If anemones you don't like, why not plant roses instead?

Im Deutschen dagegen spielt die Interpretationsdomäne bei der Lizenzierung von V2-Stellung keine Rolle. Auch syntaktisch und semantisch desintegrierte Konditionalsätze blockieren V2- 


\begin{tabular}{|l||c|c|}
\hline Nebensatztyp & V2 Deutsch & Voranstell. Engl. \\
\hline \hline Komplementsatz, Brückenverb & $+/-$ & $+/-$ \\
\hline Semifaktiver Komplementsatz & $+/-$ & $+/-$ \\
\hline Faktiver Komplementsatz & - & - \\
\hline Temporaler Adverbialsatz & - & $+/-$ \\
\hline Kausaler Adverbialsatz (weil) & $+/-$ & $-/-$ \\
\hline Appositiver Relativsatz & - & $+/-$ \\
\hline $\begin{array}{l}\text { Restriktiver Relativsatz, definites Anteze- } \\
\text { dens }\end{array}$ & - & $+/-$ \\
\hline $\begin{array}{l}\text { Restriktiver Relativsatz, indefinites Anteze- } \\
\text { dens }\end{array}$ & + & \\
\hline
\end{tabular}

Tabelle 7.3.: Lizenzierungskontexte im Vergleich: Deutsch und Englisch

Da die untersuchten Wurzelphänomene im Englischen und im Deutschen nicht in denselben Kontexten auftreten, können wir davon ausgehen, dass den beiden Phänomenen unterschiedliche Lizenzierungsbedingungen zugrunde liegen. Bezogen auf die Distribution von V2-Stellung im Deutschen wurde argumentiert, dass diese nur in Nebensätzen, welche [+at-issue] sind, möglich ist. Im Englischen können Voranstellungstransformationen aber auch in appositiven Relativsätzen auftreten, also in einem Kontext, der im Deutschen eindeutig [-at-issue] ist. Dass ein appositiver Relativsatz auch im Englischen nicht dazu verwendet werden kann, ohne seinen Bezugssatz [+at-issue]-Inhalt auszudrücken, belegt das folgende Beispiel von Beaver (2012):

Q: Where's Bob these days?

B: \#Bob, who is in Austin, hasn't called me for a week.

Im Deutschen können lediglich die Nebensätze aus Gruppe 1, welche je nach Kontext auch [+at-issue]-Inhalt ausdrücken, V2-Stellung aufweisen. Im Englischen dagegen liegt mit appositiven Relativsätzen ein Nebensatztyp vor, der zu Gruppe 2 zählt, da er nicht dazu verwendet werden kann, die aktuell relevante $\mathrm{Q}$ zu beantworten, und dennoch Wurzelphänomene aufweisen kann. Daraus können wir schließen, dass die At-issueness-Hypothese bezogen auf die Distribution von Topikalisierungen und Linksversetzungen im Englischen keine Gültigkeit besitzt.

Die Nebensätze, welche im Englischen Wurzelphänomene zulassen, teilen sich anders als im Deutschen zwar nicht das Merkmal [+at-issue], es handelt sich jedoch immer um Nebensätze, welche nicht grundsätzlich präsupponiert sind. Appositive Relativsätze projizieren zwar unter Einbettung, mit Projektionstests werden jedoch keine Präsuppositionen, sondern [-at-issue]-Inferenzen ermittelt, wie wir in Abschnitt

Stellung, wie das folgende Beispiel belegt (vgl. zu Sprechakt-Konditionalen im Deutschen u.a. Günthner 1999a, Scheffler 2008 und Wöllstein/Reis 2010).

(ii) *Wenn du hast Hunger - im Kühlschrank ist noch Pizza. 
3.1 gesehen haben. In Abschnitt 7.1.2 wurde erläutert, dass die Assertionshypothese auf zwei unterschiedliche Arten ausgelegt werden kann (vgl. die beiden Hypothesen in (233)). So lässt die Assertionshypothese in ihrer ursprünglichen Form nach Hooper/Thompson (1973) (vgl. (227)) offen, ob Wurzelphänomene in abhängigen Kontexten, die [-präsupponiert] sind, lizenziert werden oder aber, und dann liegt eine restriktivere Form der Assertionshypothese vor, ob nur Kontexte, die [+assertiv] sind, Wurzelphänomene aufweisen können. Ein Blick auf die Nebensatztypen, welche im Englischen Voranstellungstransformationen aufweisen können, zeigt uns, dass die Assertionshypothese in ihrer weniger restriktiven Form in der Lage ist, die Distribution von Wurzelphänomenen im Englischen korrekt zu erfassen. Die der Distribution von eingebetteten Wurzelphänomenen im Englischen zugrundeliegende Regel kann demnach wie folgt zusammengefasst werden: ${ }^{59}$

\section{Regel zur Lizenzierung von Voranstellungstransformationen im Englischen}

Voranstellungstransformationen sind in allen Nebensätzen, die [-präsupponiert] sind, möglich.

Die in (293) formulierte Regel wird der Tatsache gerecht, dass Temporalsätze, faktive Komplementsätze und restriktive Relativsätze mit einem definiten Antezedens im Englischen keine Wurzelphänomene aufweisen können, da diese drei Nebensatztypen grundsätzlich präsupponiert sind (vgl. dazu Abschnitt 4.2). Dagegen wäre eine Auslegung der Assertionshypothese, derzufolge nur assertive Nebensätze Wurzelphänomene zulassen, für das Englische zu restriktiv, da mit appositiven Relativsätzen ein Kontext vorliegt, der Wurzelphänomene aufweisen kann, ohne assertiert zu sein.

Es hat sich gezeigt, dass die Distribution von Wurzelphänomenen im Englischen weniger stark restringiert ist als im Deutschen, da im Englischen mehr Kontexte hauptsatztypische Transformationen aufweisen können. Im Englischen umfassen die Lizenzierungskontexte alle Nebensätze, die [-präsupponiert] sind, welche in der folgenden Abbildung grau eingefärbt sind. Die Kontexte, die im Deutschen V2-Stellung aufweisen können, bilden davon eine Untermenge - damit ein Nebensatz im Deutschen mit V2-Stellung gebildet werden kann, muss er nicht nur [-präsupponiert], sondern zusätzlich auch noch [+at-issue] sein, weswegen appositive Relativsätze nur mit VL-Stellung wohlgeformt sind. Die Lizenzierungskontexte für V2-Stellung in deutschen Nebensätzen sind in Abbildung 7.1 gestrichelt dargestellt.

Wir können festhalten, dass die Assertionshypothese in ihrer Ausprägung 1 (vgl. die beiden Hypothesen in (233)) das Auftreten von eingebetteten Wurzelphänomenen im Englischen korrekt erfasst. Nicht zutreffend ist dagegen die häufig unternommene Übertragung der von Hooper/Thompson (1973) für das Englische entwickelten

\footnotetext{
${ }^{59}$ In diesem Abschnitt wurde nur die Distribution von Voranstellungstransformationen im Englischen untersucht. Andere Wurzelphänomene wie beispielsweise Rechtsversetzungen treten im Englischen jedoch in den gleichen Kontexten auf (für einen Überblick vgl. Hooper/Thompson 1973), so dass wir davon ausgehen können, dass sie derselben Lizenzierungsbedingung unterliegen.
} 


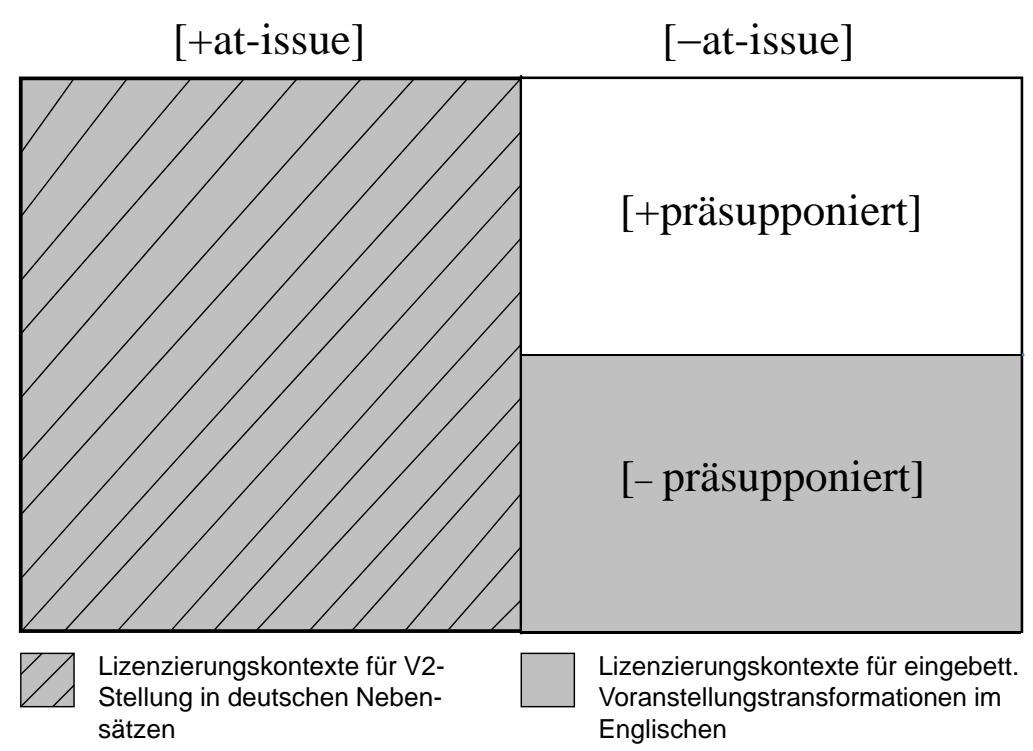

Abbildung 7.1.: Lizenzierungskontexte für Wurzelphänomene im Deutschen und im Englischen

Assertionshypothese auf die Lizenzierung von V2-Stellung im Deutschen. Zu einer solchen Übertragung hat sicherlich die Tatsache beigetragen, dass sich die Kontexte, welche Wurzelphänomene zulassen, im Deutschen und im Englischen sehr ähneln. Dies betrifft vor allem Komplementsätze, die sich bezogen auf das Auftreten von Wurzelphänomenen in beiden Sprachen gleich verhalten. Wird davon ausgegangen, dass beiden Sprachen dieselben Lizenzierungsbedingungen zugrunde liegen, vernachlässigt man jedoch Relativsätze, bei deren Betrachtung sich ein entscheidender Unterschied ergibt: Während appositive Relativsätze im Englischen Voranstellungstransformationen untergehen können, ist im Deutschen in diesem Kontext V2-Stellung blockiert. Daraus wurde geschlossen, dass es im Englischen für einen Nebensatz ausreichend ist, [-präsupponiert] zu sein, um Wurzeltransformationen aufweisen zu können, im Gegensatz dazu muss ein deutscher V2-Nebensatz zusätzlich das Merkmal [+at-issue] tragen.

\subsubsection{Unintegrierte Verbletztsätze}

Dass sich V2-Nebensätze durch einen höheren Grad an syntaktischer Desintegration als ihre kanonischen VL-Gegenstücke auszeichnen, ist von der Forschung bereits gut dokumentiert (vgl. dazu auch Abschnitt 6.4). Neben V2-Stellung führen im Deutschen aber noch weitere Faktoren zur Desintegration eines Satzes, nach Reis (1997) bemisst sich der Integrationsgrad eines Satzes dabei vor allem an seiner prosodischen (Un)Integriertheit, seiner informationsstrukturellen und illokutionären (Un)Selbstständigkeit, sowie der Möglichkeit von Variablenbindung. Auch VL-Sätze können im Deutschen unintegriert auftreten und verhalten sich dann in vielen Punk- 
ten wie abhängige V2-Sätze. Die wichtigsten Eigenschaften von unintegrierten VLSätzen sowie die Konsequenzen, die sich daraus für die At-issueness-Hypothese ergeben, sollen im Folgenden vor allem am Beispiel der unintegrierten weil-VL-Sätze (im Folgenden uWVL) diskutiert werden, die zuletzt von Reis (2013) ausführlich beschrieben wurden.

In ihrer Replik auf Antomo/Steinbach (2010) zeigt Reis (2013) überzeugend, dass sich uWVL in den zentralen Punkten wie weil-V2-Sätze verhalten: Sie können neben der propositionalen auch eine epistemische ((294-a)) oder sprechaktbezogene Begründung ausdrücken ((294-b)), können sprecherbezogene Ausdrücke wie Modalpartikeln, Einstellungsausdrücke oder Performativanzeiger enthalten ((294-c)), stehen nicht im Skopus der Matrixnegation ((294-d)), Integration in den Matrixsprechakt führt zu Ungrammatikalität ((294-e)) und Quantoren-Variablen-Bindung ist blockiert ((294-f)), um nur die wichtigsten Eigenschaften zu nennen. Zudem sind uWVL intonatorisch und syntaktisch unintegriert, woraus Reis (2013) insgesamt schließt, dass es sich um Wurzelsätze mit einem assertiven Potential handelt (Beispiel (294-c) nach Reis 2013, 251 und Beispiele (294-d) bis (294-f) nach Reis 2013, 245).

(294) a. Er ist nicht da, weil (nämlich) kein Licht brennt.

b. Brauchst du noch was? Weil ich jetzt einkaufen gehe.

c. Sie KÖNnen mich gar nicht mehr belangen, weil ich nämlich hiermit KÜNdige.

d. Er ist nicht da, weil (nämlich) kein Licht brennt, (*sondern weil abgeschlossen ist).

e. *Ist er nicht da, weil (nämlich) kein Licht brennt?

f. ${ }^{*}$ Keiner $_{i}$ trinkt, weil seine ${ }_{i}$ Freundin (nämlich) fremdgeht.

Dass sich uWVL in allen relevanten Punkten wie weil-V2-Sätze verhalten, zeigt laut Reis (2013), dass V2-Stellung, anders als von Antomo/Steinbach (2010) behauptet, weder für die Lizenzierung der desintegrierten Lesarten, noch für das assertive Potential von weil-V2-Sätzen verantwortlich sein kann. Stattdessen führt Reis (2013) die gemeinsamen Eigenschaften von weil-V2-Sätzen und uWVL auf zwei Faktoren zurück: Die syntaktisch-prosodische Desintegration von weil-V2-Sätzen und uWVL lizenziert die epistemische und sprechaktbezogene Lesart und ist verantwortlich dafür, dass uWVL und weil-V2-Sätze nicht eingebettet auftreten können. Auf der anderen Seite ist in den Selektionsbedingungen des zweistelligen Konnektors weil festgelegt, dass das Explanans-Argument durch eine Größe realisiert wird, „die assertive Geltung zulässt" (Reis 2013, 225), worauf das assertive Potential von uWVL, aber auch das von weil-V2-Sätzen zurückgeführt werden kann. Dass Assertivität nicht mit V2-Stellung korreliert, wurde in Abgrenzung zu der in u.a. Antomo/Steinbach (2010) vertretenen Assertionshypothese auch in Abschnitt 7.2 gezeigt: Am Beispiel der freien dass-Sätze, die genau wie uWVL typische Eigenschaften von Assertionen aufweisen, wurde in diesem Abschnitt argumentiert, dass Assertivität nicht auf V2-Stellung beschränkt ist, umgekehrt wurde gezeigt, dass 
nicht alle abhängigen V2-Sätze assertiv sind.

Am Beispiel der uWVL zeigt sich, dass sich unintegrierte VL-Sätze in zahlreichen Punkten genau wie abhängige V2-Sätze verhalten. Sie lizenzieren dieselben Lesarten, können ein assertives Potential aufweisen und unterliegen denselben Stellungsrestriktionen wie V2-Nebensätze. ${ }^{60}$ Aber bedeutet das auch, dass sich unintegrierte VL-Sätze diskurssemantisch wie V2-Nebensätze verhalten und immer ohne ihren Bezugssatz [+at-issue]-Inhalt ausdrücken? Anders als V2-Stellung korreliert Desintegration nicht mit At-issueness, wie die folgenden Beobachtungen zeigen:

In Abschnitt 2.2.4 wurde gezeigt, dass appositive Relativsätze grundsätzlich nicht dazu geeignet sind, ohne ihren Bezugssatz [+at-issue]-Informationen auszudrücken (vgl. dazu auch Bartsch 1978, 8, Hartmann 1984, 313f und Holler 2009). Gleichzeitig beobachtet u.a. Holler $(2013,274)$, dass sich nicht-restriktive Relativsätze im Gegensatz zu restriktiven „wie selbstständige Sätze (verhalten): Sie sind beispielsweise opak bzgl. quantifikationeller Bindung und Negation“. Holler (2005) zeigt zudem, dass nicht-restriktive Relativsätze typische Eigenschaften von selbstständigen, nicht integrierten Sätzen aufweisen, indem sie beispielsweise Satzadverbien oder Partikeln zum Ausdruck der Sprechereinstellung wie angeblich, ja oder leider enthalten können. Auch informationsstrukturell sind appositive Relativsätze selbstständig und weisen eine eigene FHG auf. Dementsprechend schlagen Holler (2005) und Reis (1997) eine syntaktische Analyse vor, bei der nicht-restriktive Relativsätze quasiverwaist sind und mit dem übergeordneten Satz erst an der höchstmöglichen Stelle verbunden werden. Appositive Relativsätze sind folglich nicht in die syntaktische Struktur ihres Bezugssatzes eingebunden und demnach unintegriert. Dass sie dennoch nicht zum Ausdruck von [+at-issue]-Informationen geeignet sind (sondern stattdessen eine N-UDQ beantworten, wie u.a. Beaver 2012 und Onea 2013 annehmen, vgl. dazu Abschnitt 2.1.2), deutet darauf hin, dass Desintegration nicht mit At-issueness korreliert.

Ein weiterer Punkt, der belegt, dass Desintegration nicht mit At-issueness korreliert, betrifft Komplementsätze von Verben. Diese sind als obligatorische Argumente des Matrixprädikats unabhängig davon, ob sie [+at-issue]-Information ausdrücken oder

\footnotetext{
${ }^{60}$ Auch wenn Reis (2013) bezogen auf $u W V L$ zeigt, dass sich diese wie weil-V2-Sätze verhalten, ist bislang jedoch noch nicht eindeutig untersucht worden, ob diese Beobachtung auf alle V2Nebensätze und die entsprechenden unintegrierten VL-Sätze generalisiert werden kann. Bezogen auf Konzessivsätze mit obwohl zeigen Antomo/Steinbach (2013), dass unintegrierte obwohl-VLSätze nicht in jedem Kontext dieselbe Interpretation erhalten wie obwohl-V2-Sätze. So führen unintegrierte obwohl-VL-Sätze zwar genau wie obwohl-V2-Sätze zu einer Infragestellung der im Bezugssatz ausgedrückten Proposition, wenn sie nachgestellt sind, sie sind jedoch semantisch affirmierend, wenn sie ihrem Bezugssatz vorangehen. Obwohl-V2-Sätze können wie alle abhängigen V2-Sätze in diesem Kontext nicht verwendet werden und sind demnach nie affirmierend.
}

(i) a. Ich nehme das Auto. Obwohl: Die Parksituation ist ja extrem schlecht.
b. Ich nehme das Auto. Obwohl die Parksituation ja extrem schlecht ist...
c. Obwohl die Parksituation extrem schlecht ist: Ich nehme das Auto.
d. *Obwohl: Die Parksituation ist extrem schlecht: Ich nehme das Auto. 
nicht, immer in die syntaktische Struktur ihres Bezugssatzes integriert. Auch wenn ein VL-Komplementsatz darauf abzielt, die QUD zu beantworten, muss der abhängige Satz prosodisch, syntaktisch und informationsstrukturell in seinen Bezugssatz integriert sein, wie das folgende ungrammatische Beispiel belegt.

$$
\begin{aligned}
& \text { Q: Wo ist Maria? } \\
& \text { B: *Peter glaubt. ( } \backslash) \text { Sie ist in Paris. }
\end{aligned}
$$

Auch Komplementsätze mit V2-Stellung sind intonatorisch integriert und erlauben Quantoren-Variablen-Bindung (vgl. Beispiel (296-a) nach Meinunger 2004, 314), zudem stehen sie im Skopus des Matrixsprechaktoperators, weshalb sie auch in einen Interrogativsatz integriert werden können, wie Antomo/Steinbach $(2010,8)$ anhand von Beispiel (296-b) zeigen.

a. $\operatorname{Jeder}_{i}$ denkt, er ${ }_{i}$ kommt zu kurz.

b. Hat Peter geglaubt, Maria wird wieder mit dem Rauchen anfangen?

Die Daten zeigen, dass V2-Komplementsätze eindeutig in die syntaktische Struktur ihres Matrixsatzes integriert sind, weswegen Meinunger (2004) die abhängigen V2Sätze als hybride Strukturen analysiert, welche als $\mathrm{V}^{0}$-Komplemente basisgeneriert werden, bevor sie obligatorisch extraponiert werden müssen (vgl. für eine ähnliche Analyse Truckenbrodt 2006). Wir können festhalten, dass Komplementsätze, obwohl sie grundsätzlich zum Ausdruck von [+at-issue]-Informationen geeignet sind, nicht unintegriert auftreten können. Selbst wenn sie V2-Stellung aufweisen, sind sie als interne $\mathrm{V}^{0}$-Argumente syntaktisch in die Matrixstruktur integriert, wenngleich auch weniger stark als die kanonisch integrierten VL-Komplementsätze.

Die Beispiele zeigen, dass Desintegration und At-issueness nicht grundsätzlich korrelieren. Während mit appositiven Relativsätzen unintegrierte Sätze vorliegen, welche ohne ihren Bezugssatz nur eine N-UDQ, nicht aber die aktuell relevante QUD, beantworten können, können Komplementsätze grundsätzlich nicht unintegriert auftreten, auch dann nicht, wenn sie ohne ihren Bezugssatz [+at-issue]-Inhalt ausdrücken. Im Gegensatz zu V2-Stellung, welche nur abhängige Sätze, die [+at-issue] sind, (optional) aufweisen können, handelt es sich bei syntaktischer Desintegration somit um kein grundsätzliches Merkmal zur Markierung von At-issueness. Nicht syntaktische Desintegration bzw. V2-Stellung, sondern At-issueness führt zu den in Abschnitt 3 beschriebenen Eigenschaften, die sich alle Nebensätze aus Gruppe 1 teilen, d.h. Sensibilität für einen direkten Widerspruch sowie keine grundsätzliche Projektion unter Einbettung. Dass sich auch alle abhängigen V2-Sätze durch diese Eigenschaften auszeichnen, hängt damit zusammen, dass es sich bei V2-Stellung um einen optionalen Markierer für At-issueness handelt, V2-Nebensätze also immer [+at-issue]-Inhalt ausdrücken. Grundsätzlich können aber auch kanonisch integrierte VL-Sätze ohne ihren Bezugssatz die jeweils aktuelle QUD beantworten, wie in Abschnitt 2.2 gezeigt wurde.

Weist ein abhängiger Satz V2-Stellung auf, damit seine Proposition als [+at-issue] ausgewiesen wird, führt dies als Nebeneffekt zu einer stärkeren Desintegration des 


\section{Lizenzierungsbedingungen}

Nebensatzes. Als Folge davon, sind V2-Nebensätze genau wie unintegrierte VLNebensätze zum Ausdruck von besonderen Lesarten wie der epistemischen oder sprechaktbezogenen Interpretation geeignet.

\subsection{Zusammenfassung des Kapitels}

Die Distribution von V2-Stellung in deutschen Nebensätzen wird in der Forschungsliteratur häufig mithilfe der ursprünglich von Hooper/Thompson (1973) fürs Englische entwickelten Assertionshypothese erklärt. In Abschnitt 7.1.2 wurde gezeigt, dass die Assertionshypothese dabei zwei Auslegungen erlaubt: In ihrer restriktiven Variante wird V2-Stellung nur in Nebensätzen, die über ein eigenes assertives Potential verfügen, lizenziert, wohingegen die weniger restriktive Version davon ausgeht, dass alle nicht-präsupponierten Nebensätze mit V2-Stellung gebildet werden können. Es wurde argumentiert, dass beide Auslegungsmöglichkeiten mit Problemen verbunden sind. Dass das Merkmal [-präsupponiert] für das Auftreten von V2-Stellung nicht ausreichend ist, belegen appositive Relativsätze, welche nur mit VL-Stellung wohlgeformt sind, obwohl sie nicht präsupponiert sein dürfen. Wird dagegen davon ausgegangen, dass nur Nebensätze mit einem eigenen assertiven Illokutionspotential V2-Stellung lizenzieren, ist diese Auslegung zu restriktiv. So wurde gezeigt, dass Komplement- und Relativsätze mit V2-Stellung in die Matrixillokution integriert sind, woraus wir ableiten können, dass ein eigenes assertives Potential keine notwendige Bedingung für das Auftreten von V2-Stellung darstellt. Zudem liegen mit freien dass-Sätzen Strukturen vor, die mehr Ähnlichkeiten mit Assertionen aufweisen als V2-Komplementsätze und dennoch nicht mit V2-Stellung gebildet werden können.

Durch die Übertragung der Assertionshypothese auf die Distribution von V2-Stellung in deutschen Nebensätzen können demnach nicht alle relevanten Kontexte korrekt erfasst werden. Aus diesem Grund wurde in Abschnitt 7.2 dafür argumentiert, dass V2-Stellung nicht durch Assertivität, sondern durch das Konzept der At-issueness lizenziert wird. Im Deutschen kann beobachtet werden, dass nur die Nebensätze aus Gruppe 1, welche dazu verwendet werden können, die aktuell relevante Q zu beantworten, V2-Stellung aufweisen können. Diese Nebensätze sind prinzipiell ambig, da sie je nach Kontext entweder [+at-issue]- oder [-at-issue]-Inhalt ausdrücken. V2Stellung dient der Disambiguierung, indem signalisiert wird, dass der entsprechende Nebensatz darauf abzielt, Q zu beantworten. Da auf diese Weise auch Rückschlüsse gezogen werden können, wie die anvisierte Q lautet, ist zu erwarten, dass V2-Stellung besonders häufig dann auftritt, wenn Q nicht explizit geäußert wird. Diese Vorhersage muss durch zukünftige Untersuchungen allerdings noch überprüft werden. Die Beobachtung, dass präsupponierte Nebensätze im Deutschen nicht mit V2-Stellung gebildet werden können, ergibt sich ebenfalls aus der At-issueness-Hypothese. So wurde in Kapitel 4 dafür argumentiert, dass Präsuppositionen grundsätzlich nicht dazu geeignet sind, [+at-issue]-Inhalt auszudrücken, folglich lizenzieren sie auch keine V2-Stellung. 
In Abschnitt 7.3 wurde die At-issueness-Hypothese auf weitere Anwendungsbereiche bezogen. Es hat sich gezeigt, dass obwohl-Sätze mit V2-Stellung immer [+at-issue]Inhalt ausdrücken, da sie eine Unterfrage der jeweils relevanten QUD beantworten und damit die im Bezugssatz ausgedrückte Antwort auf die QUD infragestellen bzw., je nach Kontext, revidieren. Außerdem wurde argumentiert, dass auch Konditionalsätze zur Hauptstruktur gehören können. Dass wenn-Sätze dennoch nicht mit V2-Stellung gebildet werden können, wurde auf eine lexikalische Ausdifferenzierung im konditionalen Bereich zurückgeführt - so sind andere konditionale Satzeinleiter wie vorausgesetzt oder angenommen durchaus in der Lage, einen V2-Satz einzuleiten. Ob die At-issueness-Hypothese auch die Distribution von abhängiger V2-Stellung in anderen Limited Embedded V2-Sprachen erfasst, musste dagegen offen bleiben.

Eine kurze Untersuchung des Auftretens von Linksversetzungen und Topikalisierungen in englischen Nebensätzen in Abschnitt 7.3.2 hat gezeigt, dass diese nicht nur in [+at-issue]-Sätzen auftreten können, sondern in allen Nebensätzen, die nicht präsupponiert sind. Somit trifft die Assertionshypothese in ihrer weniger restriktiven Form anders als im Deutschen auf die Lizenzierung von eingebetteten Wurzelphänomenen im Englischen zu. Abschließend konnte gezeigt werden, dass Desintegration eines abhängigen Satzes im Deutschen nicht mit At-issueness korreliert - so existieren einerseits unintegrierte Sätze, die nicht zum Ausdruck von [+at-issue]-Informationen geeignet sind, umgekehrt können auch kanonisch integrierte Nebensätze Teil der Hauptstruktur sein. 
7. Lizenzierungsbedingungen 


\section{Teil III.}

\section{Temporale und kausale Adverbialsätze im Vergleich}





\section{Relationale und nicht-relationale Adverbiale}

Sowohl Temporal- als auch weil-Sätze liefern zusätzliche Informationen bezogen auf das im Matrixsatz ausgedrückte Ereignis. Beide Arten von Nebensätzen sind optional und werden syntaktisch als Adjunkte realisiert. Trotz dieser Gemeinsamkeiten existieren aber auch wesentliche Unterschiede zwischen den beiden Nebensatztypen. Was die morphosyntaktische Form betrifft, wurde in Kapitel 6 gezeigt, dass Temporalsätze grundsätzlich nicht mit V2-Stellung gebildet werden können, weil-Sätze dagegen schon. Weitere Unterschiede zwischen temporalen und kausalen Adverbialsätzen wurden in den Kapiteln 2.2 und 3 diskutiert. So haben wir gesehen, dass Temporalsätze anders als weil-Sätze nie [+at-issue]-Inhalt ausdrücken können. Damit hängt auch die Tatsache zusammen, dass Temporalsätze unter Einbettung projizieren und ihr Inhalt nur mithilfe besonderer Strategien hinterfragt werden kann, was ebenfalls nicht auf die Gesamtheit aller Adverbialsätze zutrifft. Schließlich wurde dafür argumentiert, dass Temporalsätze im Gegensatz zu weil-Sätzen immer präsupponiert sind (vgl. dazu Abschnitt 4.2).

Mit Temporal- und weil-Sätzen liegen demnach zwei Nebensatztypen vor, die sich insbesondere bezogen auf die Fähigkeit, [+at-issue]-Inhalt auszudrücken und die damit verbundene Eigenschaft, V2-Stellung zuzulassen, grundsätzlich voneinander unterscheiden, weswegen weil-Sätze zu Gruppe 1 und Temporalsätze zu Gruppe 2 gezählt wurden. Am Beispiel dieser beiden Nebensatztypen soll in den folgenden Abschnitten nun versucht werden, diese formalen und funktionalen Unterschiede auf einen grundlegenden semantischen Unterschied zwischen temporalen Subjunktionen und weil zurückzuführen.

Die bisher genannten Unterschiede zwischen temporalen und anderen Adverbialsatztypen betreffen die morphosyntaktische Form sowie die diskurssemantische Funktion der Sätze. Bisher nicht betrachtet wurde dagegen die Funktion eines Adverbialsatzes in Bezug auf seinen Matrixsatz, also die intrasententiale Semantik. Auch in diesem Punkt unterscheiden sich Temporalsätze von weil-Sätzen. In diesem Kapitel werden nun Unterschiede zwischen den beiden Adverbialsatztypen diskutiert, welche allesamt darauf hinweisen, dass sich Temporal- und weil-Sätze in der Art ihres semantischen Beitrags grundsätzlich voneinander unterscheiden. Als Ergebnis dieser Gegenüberstellung der beiden Adverbialsatztypen wird aus diesem Kapitel hervorgehen, dass Subjunktionen, die einen Adverbialsatz einleiten, entweder relational, d.h. zweistellig, oder nicht-relational, also einstellig, sein können. Zur ersten Gruppe zählt kausales weil, wohingegen gezeigt wird, dass temporale Subjunktionen nur ein Argument nehmen. Ein Vorteil dieser Analyse besteht darin, dass die in den 


\section{Relationale und nicht-relationale Adverbiale}

vorherigen Abschnitten festgestellten Unterschiede zwischen den beiden Adverbialsatztypen auf eine grundlegende semantische Differenz zwischen temporalen Subjunktionen und kausalem weil zurückgeführt werden können, was Gegenstand von Kapitel 9 sein wird. Da es in Kapitel 9 vor allem um die Klärung der Frage geht, weshalb temporale Nebensätze immer [-at-issue]-Inhalt ausdrücken und aus diesem Grund nie V2-Stellung aufweisen können, werden Temporalsätze im Folgenden ausführlicher behandelt als Kausalsätze. Wenn in den folgenden Abschnitten von kausalen Adverbialsätzen die Rede ist, sind damit ausschließlich weil-Sätze gemeint. Ob die im Folgenden vorgeschlagene Analyse auch auf andere kausale Konnektoren wie da oder denn übertragen werden kann, ist noch zu überprüfen.

Bereits in Abschnitt 2.2.1 haben wir gesehen, dass es sich bei dem Ausdruck weil um einen zweistelligen Konnektor des Typs $<\mathrm{t},<\mathrm{t}, \mathrm{t}>>$ handelt, der eine kausale Relation zwischen den beiden durch ihn verknüpften Sätzen herstellt. Auch temporale Subjunktionen werden traditionell als zweistellige Konnektoren klassifiziert, die ausdrücken, dass zwischen ihren beiden sententialen Argumenten entweder eine Relation der Vor-, Nach- oder Gleichzeitigkeit besteht (vgl. für eine solche relationale Analyse von temporalen Subjunktionen beispielsweise Blühdorn 2004b, Buscha 1989, 452, Eisenberg 1989, Eisenberg et al. 2009, Helbig/Buscha 1986, 454, Heinämäki 1978, Lohnstein 2004, Max 2004, Pasch et al. 2003, Schilder 2004 und de Swart 1991). In seiner Dissertation zeigt Johnston (1994) jedoch, dass sich Temporalsätze im Englischen weder in negierten noch in quantifizierten Strukturen wie relationale Ausdrücke verhalten. Im Folgenden werden wir sehen, dass dies auch für das Deutsche gilt, wobei die Interaktion von Temporal- und weil-Sätzen in negierten Strukturen in Abschnitt 8.1 und in quantifizierten Strukturen in Abschnitt 8.2 untersucht wird. Im Zentrum von Kapitel 8.3 steht das Verhalten der beiden Adverbialsatztypen in Kopulakonstruktionen.

\subsection{Interaktion mit Negation}

Ein wesentlicher Punkt, in dem sich weil-Sätze von Temporalsätzen unterscheiden, betrifft ihr Verhalten in Bezug auf einen negierten Matrixsatz. Enthält der Bezugssatz eines weil-Satzes eine Negation, ist der Gesamtsatz ambig. Wie in zahlreichen Arbeiten beobachtet, hat eine Äußerung wie die folgende zwei Lesarten:

Daniel ist nicht nach Italien gefahren, weil er sich erholen will.

Die Negation kann sich auf die kausale Relation zwischen den beiden Teilsätzen beziehen. In diesem Fall ist Daniel nach Italien gefahren, der Grund für die Reise ist aber nicht die erhofte Erholung, sondern beispielsweise die Teilnahme an einer Tagung. Die entsprechende Struktur ist in (298-a) dargestellt. Der Kausalsatz kann aber auch außerhalb des Skopus der Matrixnegation interpretiert werden (vgl. (298-b)). Meistens wird dies begleitet durch eine kurze Sprechpause vor dem Kausalsatz sowie fallender Intonation am Ende des Matrixsatzes. In diesem Fall ist Daniel anstatt nach Italien zu fahren, zu Hause geblieben, um sich zu erholen. 


$$
\begin{aligned}
& \text { a. } \neg \text { weil' }(p, q) \\
& \text { b. weil' }(p, \neg q)
\end{aligned}
$$

Die beiden Lesarten können durch unterschiedliche Strategien voneinander isoliert werden. Beispielsweise kann ein weil-Satz mit V2-Stellung nur außerhalb der Matrixnegation interpretiert werden, Lesart (298-a) ist nicht möglich. Steht der Kausalsatz wie in dem folgenden Beispiel im Vorfeld, kommt ebenfalls nur Lesart (298-b) in Frage.

(299) Weil Daniel sich erholen will, ist er nicht nach Italien gefahren.

Enthält der Matrixsatz dagegen ein Korrelat im Mittelfeld, kann sich die Negation nur auf die Kausalrelation beziehen (vgl. (300-a)). Dasselbe gilt, wenn der Satz mit sondern weitergeführt wird.

a. Daniel ist nicht deswegen nach Italien gefahren, weil er sich erholen will.

b. Daniel ist nicht nach Italien gefahren, weil er sich erholen will, sondern weil er dort an einer Tagung teilnimmt.

Nach Johnston (1994, 146ff) kommt diese Ambiguität durch unterschiedliche Adjunktpositionen zustande. Eine ähnliche Erklärung für den Kontrast zwischen weilSätzen mit VL- und solchen mit V2-Stellung findet sich in u.a. Antomo/Steinbach (2010), Breindl (2009), Holler (2008), Scheffler (2005) und Uhmann (1998), die ebenfalls annehmen, dass die kausalen V2-Sätze in einer höheren syntaktischen Position stehen als die syntaktisch stärker integrierten weil-VL-Sätze und aus diesem Grund nicht mehr im Skopus der Matrixnegation positioniert sind. Da die syntaktischen Details dieser Ansätze für die weitere Untersuchung nicht relevant sind, wird der Leser für mehr Informationen auf die genannten Titel verwiesen.

Was bedeutet nun diese Ambiguität von Kausalsätzen unter Negation für die Analyse des semantischen Beitrags von weil? Aus der Tatsache, dass sich die Matrixnegation entweder auf die Hauptsatzproposition oder aber auf die kausale Relation beziehen kann, folgt, dass die Subjunktion weil eine solche kausale Relation zwischen den beiden durch sie verbundenen Propositionen herstellt. Ansonsten wäre Lesart (298-a), bei der die kausale Relation negiert wird, nicht möglich. Bei der Subjunktion weil handelt es sich demnach um ein zweistelliges Prädikat des Typs $<\mathrm{t},<\mathrm{t}, \mathrm{t}>>$, das die Hauptsatz- und die Nebensatzproposition als Argumente nimmt.

Johnston (1994, 182f) beobachtet nun für das Englische, dass sich temporale Adverbialsätze unter Negation anders verhalten als kausale. Dieselbe Beobachtung lässt sich auch für das Deutsche machen: Die für Beispiel (297) ausgemachte Ambiguität tritt nicht auf, wenn ein Temporalsatz in einen negierten Satz eingebettet ist wie in dem folgenden Beispiel:

(301) Peter hat den Roman nicht fertiggestellt, bevor er krank wurde. 


\section{Relationale und nicht-relationale Adverbiale}

Anders als bei dem Hauptsatz-Kausalsatzgefüge in (297) ist es nicht möglich, Beispiel (301) so zu interpretieren, dass die Hauptsatzproposition zutrifft. Eine analoge Lesart zu (298-a), bei der sich die Negation nicht auf die Hauptsatzproposition, sondern auf die semantische Relation zwischen den beiden Sätzen bezieht, ist ausgeschlossen. Wenn die Subjunktion aber eine zweistellige temporale Relation zwischen Haupt- und Nebensatz ausdrücken würde, so müsste auch folgende Interpretation möglich sein: Peter hat den Roman fertiggestellt und er wurde krank, aber die beiden Ereignisse stehen nicht in einer Relation der Vorzeitigkeit zueinander. Dass diese Interpretation (bei unmarkierter Intonation) nicht zugänglich ist, zeigt, dass keine temporale Relation zwischen den beiden Sätzen, welche negiert werden könnte, eingeführt wird. Die entsprechende Struktur in (302) wird aus diesem Grund als ungrammatisch markiert:

$$
* \neg \text { bevor' }(p, q)
$$

Nun ließe sich einwenden, dass eine Interpretation wie (302), bei der die temporale Relation zwischen den beiden Sätzen negiert wird, sehr wohl möglich ist, wenn die Subjunktion stark betont wird wie in Beispiel (303).

$$
\text { Peter hat den Roman nicht fertiggestellt, beVOR er krank wurde. }
$$

(302) ist jedoch keine geeignete Darstellung von Satz (303). Auffallend ist, dass die temporale Subjunktion stark betont werden muss, damit die Temporalrelation (scheinbar) negiert werden kann. Bezogen auf die kausale Relation in Beispiel (297) war dies dagegen nicht nötig, mehr noch, bei Normalintonation ist (298-a) sogar die präferierte Lesart für weil-VL-Sätze. Wie sind Äußerungen wie (303) dann zu erklären? Aufgrund der starken Betonung der Subjunktion erscheint ein Ansatz aufbauend auf der Alternativensemantik für fokussierte Ausdrücke nach Rooth (1992) am vielversprechendsten (vgl. auch Rooth 1985, von Stechow 1990 sowie Kadmon 2001, 227ff für einen Überblick). Die Grundidee der Alternativensemantik besteht darin, dass fokussierte Ausdrücke neben ihrer Standardbedeutung einen zweiten, fokussemantischen Wert besitzen (vgl. dazu auch die Darstellung in Abschnitt 2.1.3). Beispielsweise denotiert ein prosodisch markierter Fokusträger wie in dem folgenden Satz zusätzlich zu seiner gewöhnlichen Bedeutung eine Menge an Alternativen. Die Konstituente, welche den Fokusakzent erhält, ist dabei Träger eines Fokusmerkmals F. Das Fokusmerkmal zeigt an, dass zu seinem Träger eine Menge an Alternativen existiert, durch welche der fokussierte Ausdruck potentiell substituiert werden könnte, und dass der fokussierte Ausdruck als ein Element dieser Alternativenmenge entnommen wurde.

(304) Peter hat $[\mathrm{MAX}]_{F}$ geschlagen.

Beispiel (304) kann nur in bestimmten Kontexten verwendet werden, da die Äußerung unter anderen Bedingungen angemessen ist als die entsprechende Äußerung ohne Fokussierung. Der Satz ist beispielsweise geeignet, um einen Kontrast zu markieren. 
Um die gewöhnliche Intension eines Ausdrucks $\alpha$ wiederzugeben, wird die Notation $\llbracket \alpha \rrbracket^{0}$ verwendet, wohingegen $\llbracket \alpha \rrbracket^{f}$ seine fokussemantische Bedeutung ausdrückt, also eine Menge an Alternativen, wie wir bereits in Abschnitt 2.1.3 gesehen haben. Der fokussierte Ausdruck Max führt beispielsweise eine Alternativenmenge wie in (305) dargestellt ein. Die jeweilige Alternativenmenge wird dabei im Wesentlichen durch Faktoren wie Kontext und Relevanz definiert. Nicht-fokussierte Ausdrücke evozieren dagegen keine Alternativen.

$$
\llbracket \operatorname{Max}_{F} \rrbracket^{f}=\{\text { Peter, Susanne, Tobias, ... }\}
$$

Kehren wir zurück zu Beispiel (303). Die stark betonte Subjunktion ist Träger eines Fokusmerkmals.

(306) Peter hat den Roman nicht fertiggestellt, $[\mathrm{beVOR}]_{F}$ er krank wurde.

Als fokussierter Ausdruck verweist betontes bevor auf eine Alternativenmenge. Je nach Kontext kann dies $\mathrm{H}$ beispielsweise signalisieren, dass eine Alternative zu bevor aus der entsprechenden Menge eingesetzt werden muss. Die Alternativenmenge für fokussiertes bevor ist in (307-a) angegeben. Nicht-fokussiertes bevor eröffnet dagegen nur eine Menge mit einem einzigen Element, wie in (307-b) dargestellt.

$$
\begin{aligned}
& \text { a. } \llbracket \text { bevor }_{F} \rrbracket^{f}=\{\text { nachdem, als }, \ldots\} \\
& \text { b. } \llbracket \text { bevor } \rrbracket^{0}=\{\text { bevor }\}
\end{aligned}
$$

Somit wird auch in Äußerung (303) nicht die temporale Relation zwischen Hauptund Nebensatz negiert. Vielmehr wird die Subjunktion fokussiert, wodurch signalisiert wird, dass eine Alternativenmenge eine Rolle spielt. Beispielsweise kann der Satz auch weitergeführt werden, indem bevor mit einem anderen Element der Alternativenmenge kontrastiert wird:

(308) Peter hat den Roman nicht fertiggestellt, beVOR er krank wurde, sondern nachDEM er krank wurde.

Sätze, in denen die temporale Subjunktion fokussiert wird, erinnern an die von insbesondere Höhle (1992) untersuchten Verum-Fokus-Konstruktionen. Auch in dem folgenden Beispiel aus Höhle (1992, 123) wird die Subjunktion betont, Höhle (1992) bezeichnet diese Fälle als C-Verum-Fokus.

(309) A: Weißt du, ob Hanna kürzlich in Rom war?

B: Ich bin sicher, DASS sie mal in Rom war, aber ob das KÜRZlich war, weiß ich nicht.

Spezifisch für Verum-Fokus ist, dass das Satzradikal häufig im Kontext vorerwähnt ist. Dies trifft ebenfalls auf einen Satz wie (303) zu. So fällt es schwer, sich einen Kontext zu überlegen, in dem das Satzmaterial von (303) nicht bereits gegeben ist, die Äußerung von (303) aber dennoch wohlgeformt ist. Vielmehr scheint es der Fall zu sein, dass Sätze, in denen die temporale Subjunktion fokussiert wird, typischerweise 


\section{Relationale und nicht-relationale Adverbiale}

in solchen Kontexten verwendet werden, in denen ein Großteil der Äußerung bereits vorerwähnt ist. Satz (303) bildet z.B. eine natürliche Antwort in dem folgenden Kurzdialog:

(310) A: Zum Glück hat Peter den Roman beendet, bevor er krank wurde.

B: Peter hat den Roman nicht beendet, beVOR er krank wurde. Er hat es erst daNACH geschafft.

Es bestehen aber auch wesentliche Unterschiede zwischen Sätzen wie (303) und Verum-Fokus-Konstruktionen. Die Betonung der Subjunktion in Beispiel (309) führt dazu, dass das Zutreffen der entsprechenden Proposition hervorgehoben wird. Höhle (1992) nimmt diesbezüglich an, dass die fokussierte Subjunktion ein VERUM-Merkmal trägt (vgl. dazu auch Büring 2006). In Beispiel (303) wird das Zutreffen der entsprechenden Proposition aber gerade negiert. Zudem unterscheidet sich der fokussierte Ausdruck bevor von Ausdrücken, die den Verum-Fokus bilden, darin, dass er eine Alternativenmenge evoziert. Die fokussierte Subjunktion dass in Beispiel (309) steht dagegen nicht im Kontrast zu anderen Alternativen.

Für eine Erklärung von Beispiel (303) unter Bezugnahme auf die Alternativensemantik spricht zudem, dass die Subjunktion weil nicht ohne Weiteres fokussiert werden kann, wenn der Kausalsatz im Skopus der Matrixnegation steht. So kann der folgende Satz nur bedingt die Lesart (298-a), hier wiederholt als (311-b), ausdrücken.

$$
\begin{aligned}
& \text { a. ?Jonas ist nicht nach Italien gefahren, WEIL er sich erholen will. } \\
& \text { b. } \neg \text { weil' }(\mathrm{p}, \mathrm{q})
\end{aligned}
$$

Dies liegt daran, dass zu weil in geringerem Maße geeignete Alternativen existieren als zu temporalen Subjunktionen, die zusammen eine Alternativenmenge bilden. In einem geeigneten Kontext kann weil allerdings mit obwohl kontrastieren:

Jonas ist nicht nach Italien gefahren, WEIL er sich erholen will, sondern obWOHL er sich erholen will. Er muss dort schließlich arbeiten.

Wir können festhalten, dass die temporale Relation zwischen Haupt- und Nebensatz nicht auf vergleichbare Weise negierbar ist wie die kausale Relation zwischen den beiden Teilsätzen in (297). Satz (303) stellt dabei kein Gegenbeispiel zu dieser Beobachtung dar. Vielmehr zeigt die Fokussierung von bevor in Verbindung mit der Negation an, dass beide Propositionen zutreffen, als Satzverknüpfung aber nicht das passende Element aus der Alternativenmenge gewählt wurde.

Aus der Beobachtung, dass Temporalsätze in Verbindung mit Negation keine Ambiguität auslösen, folgert Johnston (1994, 182f), dass temporale Subjunktionen anders als kausale keine semantische Relation einführen, welche im Skopus der Negation stehen könnte. Im Gegensatz zu weil ist eine temporale Subjunktion wie bevor einstellig und nimmt nur die von ihr eingeführte Proposition als Argument, um sie auf ein Zeitintervall abzubilden. Wie dies im Detail aussieht, wird im Zentrum von Kapitel 9.1 stehen. In diesem Abschnitt wird dann auch gezeigt, dass ein Satz wie (313) die

folgende Interpretation erhält: Für das Zeitintervall, für das gilt, dass Mailin beim 
Metzger war, existiert kein Ereignis, für das gilt, dass Jenny gelernt hat. Jenny hat nicht gelernt, während Mailin beim Metzger Peter war.

Die semantische Struktur eines Satzes wie (313) oder (301) entspricht also nicht einer relationalen Darstellung wie in (302), sondern eher einer (hier stark vereinfachten) Darstellung wie in (314), in der deutlich wird, dass der Temporalsatz einen temporalen Parameter t bzw. ein Intervall i liefert, in Bezug auf das der Matrixsatz interpretiert wird.

$$
\neg \mathrm{q}^{t}
$$

Aus dieser Analyse folgt, dass Temporalsätze die gleiche Funktion wie andere Zeitausdrücke wie beispielsweise Temporaladverbien oder Präpositionalphrasen erfüllen. Auch die Zeitausdrücke in (315) drücken einen temporalen Parameter in Form eines Intervalls aus, bezogen auf das der Satz interpretiert wird.

(315) Jenny hat heute/in den Ferien/zwischen 2 und 3 Uhr nicht gelernt.

Für die funktionale Äquivalenz von temporalen Adverbialsätzen und overten Beschreibungen eines Zeitintervalls existieren zudem noch weitere Evidenzen, die in den folgenden Abschnitten diskutiert werden.

Das unterschiedliche Verhalten von weil- und Temporalsätzen unter Negation deutet darauf hin, dass temporale Subjunktionen anders als das kausale weil keine zweistellige Relation zwischen Haupt- und Nebensatzproposition herstellen. Ein weiterer Aspekt, der in die gleiche Richtung weist, besteht in dem unterschiedlichen Verhalten der beiden Adverbialsatztypen in Bezug auf Quantoren, das im folgenden Abschnitt dargestellt wird.

\subsection{Interaktion mit Quantoren}

Johnston (1994) zeigt, dass sich Kausalsätze in quantifizierten Strukturen anders verhalten als Temporalsätze. Während letztere als Restriktion fungieren können, wird ein Quantor nie durch einen Kausalsatz restringiert. Bevor auf diesen Unterschied eingegangen wird, soll im Folgenden kurz in die Grundlagen der Quantorensemantik eingeführt werden. Betrachten wir dazu den Satz (316-a) mit der Logischen Form (316-b):

$$
\begin{aligned}
& \text { a. Alle Linguisten sind Nerds. } \\
& \text { b. } \quad \forall \mathrm{x}(\text { Linguisten }(\mathrm{x}) \rightarrow \operatorname{Nerd}(\mathrm{x}))
\end{aligned}
$$

Der Allquantor stellt eine Relation zwischen zwei Mengen her, nämlich der Menge an Nerds und der Menge an Linguisten, wobei letztere eine Teilmenge der Erstgenannten bildet. Quantoren geben demnach einen Hinweis darauf, auf welche Individuen eines Modells eine bestimmte Aussage zutrifft. Quantifizierte Strukturen sind dreigliedrig, bestehend aus einem Quantor, einer Restriktion und dem Kernbereich oder 
Nukleus, wie im Folgenden dargestellt (vgl. dazu u.a. Partee 1991 und Roberts 1995):

$$
\text { Quantor \{Restriktion\} [Nukleus] }
$$

Restriktion und Nukleus bilden die beiden Argumente des Quantors, wobei Quantor und Restriktion in der natürlichen Sprache häufig eine Konstituente bilden wie in Beispiel (316-a). Genauer gesagt wird in unserem Beispielsatz die Auswertungsdomäne des Allquantors alle auf solche Individuen beschränkt, auf die die Beschreibung Linguisten zutrifft. Es wird demnach über Individuen quantifiziert. Über diese derart ermittelten Individuen wird ausgesagt, dass auf sie das Prädikat sind Nerds zutrifft.

$$
\text { Alle }\{\text { Linguisten }\}_{\text {Restriktion }}[\text { sind Nerds }]_{\text {Nukleus }}
$$

Wie in den folgenden Beispielen dargestellt, kann ein Quantor auch unrestringiert auftreten.
a. Alle sind Nerds.
b. Everybody came to the party.

In den beiden Beispielsätzen aus dem Englischen und Deutschen ist die Restriktion nicht overt realisiert. Dennoch ist die Domäne des Quantors eingeschränkt. So würde man Beispiel (319-a) nicht so interpretieren, dass alle Menschen oder sogar Lebewesen Nerds sind, sondern, dass das Prädikat auf alle im jeweiligen Kontext relevanten Individuen zutrifft. Ebensowenig würde der Quantor in Satz (319-b) unrestringiert interpretiert werden, was zu der absurden Interpretation führen würde, dass jeder Mensch auf der Party war. Stattdessen wird die Restriktion durch den Kontext geliefert. Noch ungeklärt ist, ob es sich dabei um ein primär semantisches oder pragmatisches Phänomen handelt. ${ }^{61}$

In den bisher betrachteten Beispielen wird über Individuen quantifiziert. Neben der Quantifikation über Individuen ist auch eine Quantifikation über Ereignisbeschreibungen möglich. Der Begriff der Ereignisbeschreibung ist hier dabei sehr weit gefasst und umfasst, wie in Partee (1991) vorgeschlagen, auch Ausdrücke wie mögliche Welten, Situationen und auch Zeitintervalle, auf die es im Folgenden ankommen wird. Ein Beispiel für die Quantifikation über eine Ereignisbeschreibung bildet das Zusammenspiel von einem quantifizierenden Adverb mit einem Konditionalsatz. Lewis (1975) hat gezeigt, dass Konditionalsätze in quantifizierten Strukturen die Rolle der

\footnotetext{
${ }^{61}$ Derartige Beispiele, in denen die Restriktion nicht overt realisiert ist, sondern durch den Kontext erschlossen werden muss, verleiten u.a. von Fintel (2004a) zu der Annahme, dass quantifizierte Strukturen nicht dreigliedrig, sondern zweigliedrig sind. Im Rahmen einer solchen Analyse nimmt ein Quantor nur eine Variable, und zwar den Nukleus, als Argument. Die Restriktion tritt dagegen in Form eines anaphorischen Indexes auf, der im Kontext gesättigt werden muss. Da es in dieser Arbeit nicht primär um quantifizierte Strukturen geht, wird darauf verzichtet, die beiden Ansätze gegeneinander abzuwägen, und stattdessen die klassische dreigliedrige Struktur übernommen. Alle für diese Arbeit wesentlichen Ergebnisse ließen sich aber auch in eine zweigliedrige Struktur implementieren. Für eine ausführliche Diskussion der Vorteile einer zwei- gegenüber einer dreigliedrigen Struktur wird der Leser auf von Fintel (2004a) verwiesen.
} 
Restriktion übernehmen. So wird in dem folgenden Beispiel die Domäne des Allquantors durch den Konditionalsatz auf die Welten bzw. Situationen beschränkt, in denen es regnet. Dadurch erhalten wir die Interpretation, derzufolge Peter nicht in allen, sondern nur in solchen Situationen, in denen es regnet, Rheuma bekommt.

$$
\text { Immer, wenn es regnet, bekommt Peter Rheuma. }
$$

Wie in (321) dargestellt, realisiert der Konditionalsatz die Restriktion, während der Hauptsatz den Nukleus des Quantors bildet.

$$
\text { Immer }\{\text { wenn es regnet }\}_{\text {Restriktion }}[\text { bekommt Peter Rheuma }]_{\text {Nukleus }}
$$

Kratzer (1991) erweitert diese Analyse, indem sie zeigt, dass Konditionalsätze auch dann als Restriktion eines koverten Quantors fungieren, wenn kein overter quantifizierender Ausdruck vorhanden ist. Beispielsweise würde auch eine Äußerung wie (322-a) so interpretiert werden, dass der Konditionalsatz einen koverten Allquantor $\forall$ restringiert, wohingegen der Konditionalsatz in (322-b) die Restriktion zu einem koverten Existenzquantor $\exists$ darstellt.

a. Wenn es regnet, bekommt Peter Rheuma.

b. Wenn Peter unschuldig ist, wird er freigesprochen.

Damit entwerfen Kratzer (1991) und Lewis (1975) einen Gegenentwurf zur traditionellen Analyse der Semantik von Konditionalsätzen, derzufolge es sich bei Ausdrücken wie wenn um zweistellige Konnektoren handelt. Sie zeigen, dass Ausdücke wie if bzw. wenn keine zweistellige Relation einführen, sondern vielmehr die Funktion haben, die Domäne eines koverten oder overten Quantors über Ereignisbeschreibungen zu restringieren.

Nach dieser kurzen allgemeinen Einführung in die Theorie von Quantoren soll nun das Verhalten von Temporal- und weil-Sätzen in quantifizierten Strukturen untersucht werden. Mit Konditionalsätzen liegt bereits ein gut untersuchter Fall vor, bei dem die Domäne eines Quantors durch einen Adverbialsatz restringiert wird. Dabei wird über im weitesten Sinne Ereignisbeschreibungen quantifiziert. Johnston (1994) beobachtet nun fürs Englische, dass temporale Adverbialsätze als Quantorrestriktionen fungieren können, kausale dagegen nicht (vgl. dazu auch de Swart 1991). Auch im Deutschen lässt sich dies nachweisen. Betrachten wir hierzu ein Beispiel. Aus dem komplexen Satz (323) kann nicht geschlussfolgert werden, dass Georg immer müde ist.

Georg ist immer müde, während er Nachtschicht hat.

$\not \rightarrow$ Georg ist immer müde.

Stattdessen wird Beispiel (323) so interpretiert, dass für jede Situation, in der Georg Nachtschicht hat, eine Situation existiert, für die gilt, dass Georg müde ist. Die Menge an Situationen, für die gilt, dass Georg Nachtschicht hat, bildet demnach eine Teilmenge der Situationen, in denen Georg müde ist. Aus der Tatsache, dass 


\section{Relationale und nicht-relationale Adverbiale}

Georg ist immer müde keine logische Implikation des komplexen Satzes (323) ist, können wir schließen, dass der Quantor immer durch den Temporalsatz restringiert wird. Übertragen auf die oben erläuterte dreigliedrige Struktur bedeutet dies, dass der Temporalsatz der Restriktion entspricht, während der Matrixsatz im Nukleus des Allquantors steht.

$$
\text { Immer }\{\text { während er Nachtschicht hat }\}_{\text {Restriktion }}[\text { ist Georg müde }]_{\text {Nukleus }}
$$

Der Temporalsatz spezifiziert folglich die Auswertungsdomäne für den Hauptsatz, welche aus den Zeitintervallen besteht, in denen Georg Nachtschicht hat. Die Hauptsatzproposition wird nun innerhalb dieser durch den Nebensatz spezifizierten Domäne evaluiert. Temporalsätze quantifizieren demnach über Zeitintervalle (für eine genauere Darstellung der Denotation von Temporalsätzen vgl. Abschnitt 9.1).

Nicht nur durch während eingeleitete Sätze, sondern alle Temporalsätze sind Quantorrestriktionen. So restringiert auch der nachdem-Satz in dem folgenden Beispiel den Allquantor, wodurch dessen Domäne auf die Intervalle restringiert wird, die nach Ende von Georgs Nachtschicht liegen.

(325) Georg ist immer müde, nachdem er Nachtschicht hatte.

$\not \rightarrow$ Georg ist immer müde.

Auch bevor-Sätze fungieren als Quantorrestriktionen. Beispielsweise impliziert die folgende Äußerung nicht, dass Lukas immer Beruhigungspillen nimmt, sondern nur in solchen Intervallen, die vor einer wichtigen Prüfung liegen. Der Allquantor quantifiziert über das im Hauptsatz ausgedrückte Ereignis, aber seine Domäne ist durch den Temporalsatz auf die durch den Temporalsatz denotierten Intervalle restringiert (für eine genaue Analyse des semantischen Beitrags von bevor- und nachdem-Sätzen vgl. Abschnitt 9.1).

(326) Lukas nimmt immer Beruhigungspillen, bevor er eine wichtige Prüfung hat. t> Lukas nimmt immer Beruhigungspillen.

Ein temporaler Adverbialsatz kann demnach die Domäne eines Quantors einschränken. Ist kein overter Quantor vorhanden, restringieren Temporalsätze nach Johnston (1994) einen koverten Existenzquantor analog zur Analyse von Konditionalsätzen nach Kratzer (1991). ${ }^{62}$

Wir können also festhalten, dass Temporalsätze in quantifizierten Strukturen die Rolle der Restriktion übernehmen können, in diesem Fall wird dann über Zeitintervalle quantifiziert. Betrachten wir nun im Vergleich dazu die Interaktion von weil-Sätzen mit Quantoren.

Laut der in Diesing (1992) aufgestellten „Mapping Hypothesis“ bilden solche Elemente, die in der Logischen Form außerhalb der VP stehen, die Restriktion. Als IP-Adjunkte (vgl. u.a. Lohnstein 2004, 142) kommen demnach ebenso wie Tempo-

${ }^{62}$ Schon Lewis (1975) deutet an, dass auch temporale when-Sätze in quantifizierten Strukturen Restriktionen sind. Johnston (1994) überträgt dies auf alle Temporalsätze. 
ralsätze theoretisch auch Kausalsätze als Restriktion in Frage. Dennoch wird die Domäne eines Quantors durch einen Kausalsatz nicht restringiert, wie das folgende Beispielpaar zeigt. Aus dem komplexen Satz (327) folgt die logische Implikation, dass Georg immer müde ist. Dies bedeutet, dass der Kausalsatz anders als der Temporalsatz in Beispiel (323) nicht als Quantorrestriktion fungiert. Die Domäne des Quantors immer wird nicht durch den Kausalsatz auf die Situationen beschränkt, in denen Georg Nachtschicht hat, so dass geschlussfolgert werden kann, dass Georg zu jedem Zeitpunkt müde ist.

(327) Georg ist immer müde, weil er Nachtschicht hat.

$\rightarrow$ Georg ist immer müde.

Die Struktur von (327) ist in (328) angegeben. Während die Restriktion leer ist, bildet der Gesamtsatz den Nukleus des Allquantors.

$$
\text { Immer }\{\}_{\text {Restriktion }}[\text { Georg ist müde, weil er Nachtschicht hat }]_{\text {Nukleus }}
$$

Ein Satz wie (327) kann jedoch nicht nur wie in (328) interpretiert werden, sondern ist ambig. Beispiel (327) kann auch die folgende Lesart erhalten: Für alle Situationen, für die gilt, dass Georg müde ist, gilt, dass es wegen seiner Nachtschicht ist. In diesem Fall wird der Quantor durch den Hauptsatz restringiert und der Kausalsatz steht alleine im Nukleus:

$$
\text { Immer }\{\text { Georg ist müde }\}_{\text {Restriktion }}[\text { weil er Nachtschicht hat }]_{\text {Nukleus }}
$$

Auch in dieser Lesart fungiert der Kausalsatz nicht als Restriktion.

Temporal- und weil-Sätze verhalten sich folglich auch im Deutschen in quantifizierten Strukturen unterschiedlich. Während ein Temporalsatz die Funktion der Restriktion übernehmen kann, restringiert ein mit weil eingeleiteter Satz nicht die Domäne des Quantors. Dass temporale Adverbialsätze die Auswertungsdomäne für den modifizierten Hauptsatz auf bestimmte Zeitintervalle einschränken, beobachtet auch Lohnstein (2004, 143f). Allerdings geht Lohnstein (2004) davon aus, dass auch Kausalsätze als Restriktionen die Evaluationsdomäne des Hauptsatzes spezifizieren. Würde dies zutreffen, dann sollte jedoch der komplexe Satz (327) nicht implizieren, dass Georg immer müde ist. Da diese Implikation jedoch entsteht, können wir schließen, dass Kausalsätze nicht als Quantorrestriktionen fungieren können.

Welche Bedeutung hat die Tatsache, dass Temporalsätze Quantoren restringieren, weil-Sätze dagegen nicht, nun für die Analyse des semantischen Beitrags der beiden Adverbialsatztypen? Zwei Dinge sind hier wesentlich. Zum einen stützt dieses Ergebnis die in Kapitel 4 formulierte Hypothese, dass es sich bei Temporalsätzen um präsupponierte Ausdrücke handelt. So ist es eine gängige Ansicht, dass Quantorrestriktionen präsupponiert sind (vgl. u.a. Sawada/Larson 2004). Diese Korelation wird in Kapitel 9.1 noch genauer beschrieben.

Des Weiteren, und das ist der in diesem Kapitel zentrale Punkt, kann anhand des Verhaltens von Adverbialsätzen in quantifizierten Strukturen abgeleitet werden, ob die sie einleitenden Elemente relational sind oder nicht. Als nicht-selektive Binder 


\section{Relationale und nicht-relationale Adverbiale}

können quantifizierende Adverbien wie immer nach u.a. Partee (1991) über Individuen oder Ereignisbeschreibungen quantifizieren, wobei Partee (1991), wie weiter oben bereits erwähnt, von einem weit gefassten Begriff der Ereignisbeschreibung ausgeht, der auch Beschreibungen von möglichen Welten und Zeitintervalle umfasst. Daraus folgt nun aber, dass potentielle Restriktionen in eine dieser beiden Kategorien fallen. Da Temporalsätze als Quantorrestriktionen fungieren können, muss es sich um eine Art der Ereignisbeschreibung handeln. In Abschnitt 9.1.1 wird Johnston (1994) folgend dafür argumentiert, dass Temporalsätze Zeitintervalle beschreiben. Demnach restringieren Temporalsätze die Domäne eines Quantors auf die durch sie spezifizierten Intervalle.

Dagegen können wir aus der Tatsache, dass weil-Sätze nicht als Quantorrestriktionen fungieren können, schließen, dass sie keine Ereignisbeschreibungen bilden. Stattdessen handelt es sich um relationale Ausdrücke, die sich durch eine noch ungesättigte Argumentstelle auszeichnen. Wie bereits im vorherigen Abschnitt erläutert, ist der kausale Konnektor weil vom semantischen Typ $<\mathrm{t},<\mathrm{t}, \mathrm{t}>>$. Weil benötigt demnach zwei propositionale Argumente und bildet sie auf einen Wahrheitswert ab. Ein kausaler Adverbialsatz, also eine mit weil eingeleitete Proposition, ist folglich vom Typ $<\mathrm{t}, \mathrm{t}>$, da lediglich eine der beiden Argumentstellen gesättigt ist. Ein Ausdruck des Typs $<\mathrm{t}, \mathrm{t}>$ bildet aber keine Ereignisbeschreibung, sondern entspricht einer Funktion von Wahrheitswert zu Wahrheitswert. Als solche kann ein weil-Satz nicht als Quantorrestriktion fungieren.

Johnstons (1994) Beobachtung bezogen auf das Englische ist demnach auch für deutsche Temporal- und weil-Sätze gültig. Zudem kann festgestellt werden, dass sich im Deutschen auch temporale und kausale Präpositionalphrasen ganz ähnlich verhalten. Satz (330-c) folgt aus (330-b), nicht aber aus (330-a), woraus wir schließen können, dass die temporale PP, nicht aber die kausale, den Allquantor restringiert. ${ }^{63}$

a. Vor dem Frühstück ist Martin immer schlecht gelaunt.

b. Wegen seines Jobs ist Martin immer schlecht gelaunt.

c. Martin ist immer schlecht gelaunt.

Wie Temporalsätze können demnach auch temporale PPn als Quantorrestriktionen fungieren. Auf die funktionale Äquivalenz von Temporalsätzen und anderen Temporalausdrücken wird in Abschnitt 9.1 noch genauer eingegangen.

In diesem Abschnitt wurde gezeigt, dass Temporalsätze als Quantorrestriktionen fungieren können, weil-Sätze dagegen nicht. Da Quantoren entweder über Individuen oder Ereignisbeschreibungen quantifizieren, kann daraus geschlossen werden, dass

${ }^{63}$ Durch Umstellung wird Satz (330-b) ambig. Der folgende Satz kann entweder eine Lesart haben wie (330-b) oder eine Lesart, in der der Allquantor durch den Hauptsatz restringiert wird.

(i) Martin ist immer wegen seines Jobs schlecht gelaunt.

Die Lesart, in der der Quantor durch den Hauptsatz restringiert wird, kann so umschrieben werden: Für alle Situationen, für die gilt, dass Martin schlecht gelaunt ist, gilt, dass es wegen seines Jobs ist. 
Temporalsätze wie andere Zeitausdrücke im weitesten Sinne Ereignisbeschreibungen bzw. Beschreibungen von Zeitintervallen sind. Als solche sind sie nicht-relational, d.h. sie drücken keine semantische Relation zwischen Haupt- und Nebensatz aus. Die logische Struktur eines Hauptsatz-Temporalsatzgefüges mit einem Allquantor ist in (331) dargestellt. Hier wird deutlich, dass der Temporalsatz als Ganzer (dargestellt als p) den Quantor restringiert. Eine zweistellige temporale Relation ist nicht vorhanden.

$$
\forall\{\mathrm{p}\}[\mathrm{q}]
$$

Mit weil eingeleitete Sätze dagegen bilden keine Ereignisbeschreibungen, sondern sind relationale Ausdrücke mit einer noch offenen Argumentstelle. Anders als temporale Subjunktionen führt weil demnach eine semantische Relation zwischen den beiden verknüpften Propositionen p und q ein. Die Struktur eines Satzgefüges wie (327) ist in (332) abgebildet. Daraus wird ersichtlich, dass die zweistellige Relation weil' den quantifizierten Hauptsatz q als eins ihrer Argumente nimmt. Der Hauptsatz q bildet den Nukleus des Quantors und die Restriktion, hier dargestellt als x, ist nicht overt realisiert. Stattdessen muss sie pragmatisch aus dem Kontext bzw. dem Weltwissen erschlossen werden (vgl. dazu von Fintel 2004a). Die gesamte quantifizierte Struktur füllt folglich die externe Argumentstelle des zweistelligen Konnektors weil.

$$
\text { weil' (p, } \forall\{\mathrm{x}\}[\mathrm{q}])
$$

\subsection{Verhalten in Kopulakonstruktionen}

Die Annahme, dass temporale Subjunktionen nicht-relationale Ausdrücke darstellen, erhält zusätzliche Bestätigung durch die folgende Beobachtung. Ein weil-Satz kann nicht als Teil einer Prädikativkonstruktion verwendet werden, wie das folgende Beispiel belegt:

*Weil er uns verraten hat, ist ein guter Grund ihn umzubringen.

Die Abweichung des Beispiels kann dadurch erklärt werden, dass weil-Sätze als relationale Ausdrücke mit einer noch offenen Argumentstelle nicht funktional äquivalent sind mit nominalen Ausdrücken. Aus diesem Grund kann ein Kausalsatz nicht über die Kopula sein mit einer NP verbunden werden. Temporalsätze können dagegen genau in dieser Verwendung auftreten:

(334) Bevor er in die Mittagspause geht, ist ein guter Moment, um ihn anzusprechen.

In Beispiel (334) wird der Temporalsatz mit einer NP gleichgesetzt. Die Tatsache, dass die Äußerung anders als Beispiel (333) wohlgeformt ist, zeigt, dass die beiden Elemente der Prädikativkonstruktion funktional äquivalent sind - beide denotieren 
als nicht-relationale Ausdrücke ein Zeitintervall, was im Zentrum von Abschnitt 9.1 stehen wird.

\subsection{Zusammenfassung des Kapitels}

Aufbauend auf den Ergebnissen von Johnston (1994) für das Englische wurde in diesem Kapitel gezeigt, dass sich temporale von kausalen Adverbialsätzen grundsätzlich in der Art ihres semantischen Beitrags unterscheiden. Während das kausale weil eine zweistellige Relation zwischen den beiden verknüpften Propositionen einführt, nehmen temporale Adverbialsatzeinleiter nur die von ihnen eingeführte Proposition als Argument und bilden sie auf die Beschreibung eines Zeitintervalls ab. Somit handelt es sich bei Temporalsätzen um nicht-relationale Ausdrücke, wohingegen weil-Sätze noch eine offene Argumentstelle besitzen.

Die Taxonomie von relationalen Kausal- und nicht-relationalen Temporalsätzen wurde anhand ihres unterschiedlichen Verhaltens unter Negation, der Interaktion mit Quantoren und dem Auftreten in Kopulakonstruktionen ermittelt. In Satzgefügen mit einem Kausalsatz ist es möglich, dass sich die Matrixnegation auf die kausale Relation zwischen den beiden Teilsätzen bezieht, diese Lesart ist nicht für Temporalsätze gegeben. Als Beschreibungen von Zeitintervallen können Temporalsätze wie andere Temporalausdrücke als Quantorrestriktionen fungieren, während weil-Sätze die Domäne eines Quantors nicht einschränken. Zudem konnte gezeigt werden, dass Temporalsätze in einer Kopulakonstruktion mit einem nominalen Ausdruck gleichgesetzt werden können. Für Kausalsätze als relationale Ausdrücke ist diese Möglichkeit nicht gegeben. Eine genaue Beschreibung des semantischen Beitrags von temporalen Subjunktionen im Vergleich zum kausalen weil ist Ziel des folgenden Kapitels. 


\section{Semantische Eigenschaften von Temporal- und weil-Sätzen}

In Abschnitt 8 wurde gezeigt, dass sich Temporal- anders als weil-Sätze nicht wie relationale Ausdrücke verhalten. Es besteht demnach ein grundlegender Unterschied in der Art des semantischen Beitrags zwischen weil auf der einen und temporalen Subjunktionen auf der anderen Seite. In diesem Abschnitt wird nun genauer auf die semantischen und diskurspragmatischen Eigenschaften dieser beiden Adverbialsatztypen eingegangen und es wird untersucht, ob aus dieser grundlegenden Differenz die Unterschiede in ihrer Fähigkeit ohne ihren Bezugssatz [+at-issue]-Inhalt auszudrücken und V2-Stellung zuzulassen abgeleitet werden können. Im Zentrum von Abschnitt 9.1 werden die temporalen Adverbialsätze stehen. Dabei soll zuerst in Abschnitt 9.1.1 aufbauend auf den Ergebnissen von Johnston (1994) zum Englischen und Zifonun et al. (1997) zu deutschen Präpositionen eine einstellige Semantik von temporalen Subjunktionen entwickelt werden. Die grundlegende Idee ist dabei, dass eine temporale Subjunktion ihr Komplement auf ein Zeitintervall i abbildet, in welchem das Hauptsatzereignis enthalten sein muss. Daraus ergibt sich die Frage, auf welche Weise das im Hauptsatz ausgedrückte Ereignis innerhalb von i eingeordnet wird, die im Zentrum von Abschnitt 9.1.2 stehen wird, wo für eine pragmatische Analyse plädiert wird. In Abschnitt 9.1.3 wird schließlich untersucht, auf welche Weise die mit einem Temporalsatz verbundene Präsupposition aus der hier vorgestellten einstelligen Semantik abgeleitet werden kann, zudem erfolgt eine Modellierung im Rahmen von SDRT. Im anschließenden Teil 9.2 wird der semantische Beitrag von weil-Sätzen beschrieben. Da sich weil-Sätze in Abschnitt 8 wie relationale Ausdrücke verhalten haben und dies der traditionellen Analyse von weil entspricht, wird Abschnitt 9.2 deutlich kürzer ausfallen als der entsprechende Teil zu Temporalsätzen.

\subsection{Temporalsätze}

\subsubsection{Denotation eines Intervalls}

Zahlreiche Argumente sprechen dafür, dass temporale Subjunktionen, anders als häufig angenommen, keine zweistellige Relation wie beispielweise , $<$ ' oder ,>' ausdrücken. Vielmehr verhalten sie sich wie einstellige Operatoren. Die genaue Art ihres semantischen Beitrags wird nun in diesem Abschnitt dargestellt. Dabei richte ich mich in den wesentlichen Punkten nach der semantischen Analyse von temporalen Subjunktionen in Johnston (1994) (für einen Überblick vgl. auch Hara 2008 
und Sawada/Larson 2004). Zudem wird dafür argumentiert, dass sich temporale Subjunktionen und Präpositionen wie beispielsweise vor oder nach semantisch sehr ähnlich sind. Beide sind einstellige Ausdrücke, stellen aber dennoch eine Relation zwischen zwei Ereignisbeschreibungen her. Aus diesem Grund wird in der folgenden Darstellung des semantischen Beitrags von temporalen Subjunktionen auch Rückgriff auf die Beschreibung von Präpositionen durch Zifonun et al. (1997, 2098ff) genommen. Dass sich temporale Subjunktionen und Präpositionen semantisch sehr ähnlich sind, beobachtet auch Blühdorn (2004b, 127), der zudem darauf hinweist, dass aufgrund dieser Ähnlichkeit häufig Gleichheit oder Teilgleichheit der Wortformen vorliegt (vgl. dazu auch Eisenberg 2006, 205 und Buscha 1989, 7). Beispielsweise können im Deutschen während und seit entweder als Präposition oder als Subjunktion verwendet werden, Teilgleichheit besteht zudem zwischen den Formen nachdem und nach, sowie zwischen bevor und vor. Auch im Englischen liegen mit after und before zwei Ausdrücke vor, die sowohl einen Nebensatz als auch eine NP einleiten können, dasselbe trifft auf avant, après und pendant im Französischen zu.

Die folgende Darstellung beschränkt sich auf den zentralen semantischen Beitrag von temporalen Subjunktionen als Klasse. Nur einzelne Subjunktionen betreffende Detailstudien werden hier genauso weggelassen wie die Diskussion von nicht-temporalen Lesarten wie beispielsweise kausale Interpretationen von nachdem (vgl. hierzu u.a. Blühdorn 2004a, Lohnstein/Bredel 2004 sowie natürlich Pasch et al. 2003).

Beginnen wir mit der Erläuterung einiger grundlegender Konzepte. Zeitliche Strukturen lassen sich in Intervalle gliedern, welche selbst wiederum Mengen von Zeitpunkten sind (vgl. u.a. Ballweg 1988, Beaver/Condoravdi 2003, Copley 2009, Johnston 1994 und Reichenbach 1947). Ein Intervall kann dabei einen bis unendlich viele Zeitpunkte enthalten, was auch daran liegt, dass ein einzelner Zeitpunkt endlos in weitere, kürzere Zeitpunkte unterteilt werden kann. Ereignisbeschreibungen können nun mittels eines koverten oder overten temporalen Parameters auf einer Zeitachse verortet werden. Nehmen wir an, dass die Situationsbeschreibung s' = Jap backt einen Kuchen zum Zeitpunkt t' stattfindet. Dann können wir t' auf einem Zeitstrahl verorten, wie in Abbildung 9.1 dargestellt.

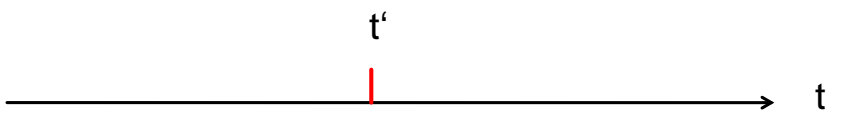

Abbildung 9.1.: Verortung einer Ereignisbeschreibung auf einem Zeitstrahl

Die Zeitspanne bzw. das Zeitintervall, das eine Ereignisbeschreibung auf dem Zeitstrahl einnimmt, wird im Folgenden als Laufzeit bezeichnet. Je nachdem, ob ein Ereignis punktuell oder durativ ist, kann dieses Intervall aus einem bis potentiell unendlich vielen Zeitpunkten bestehen. Betrachtet man eine auf dem Zeitstrahl verortete Ereignisbeschreibung, können drei benachbarte Intervalle voneinander unter- 
schieden werden. Johnston $(1994,92)$ spricht in diesem Zusammenhang vom Konzept der temporalen Nachbarschaft (temporal neighborhood). Neben der Laufzeit von s' sind dies die Vor- und die Nachzeit. Die Vorzeit von s' grenzt direkt an den Beginn der Laufzeit an und erstreckt sich in die Vergangenheit, bis sie auf eine weitere Ereignisbeschreibung mit demselben semantischen Inhalt wie s' stößt. Als Nachzeit wird dagegen das Intervall bezeichnet, welches am rechten Rand der Laufzeit beginnt und solange in die Zukunft reicht, bis es ebenfalls auf eine andere Ereignisbeschreibung mit der Beschreibung von s' trifft. Eine Übersicht der drei Intervalle ist in Grafik 9.2 dargestellt. ${ }^{64}$

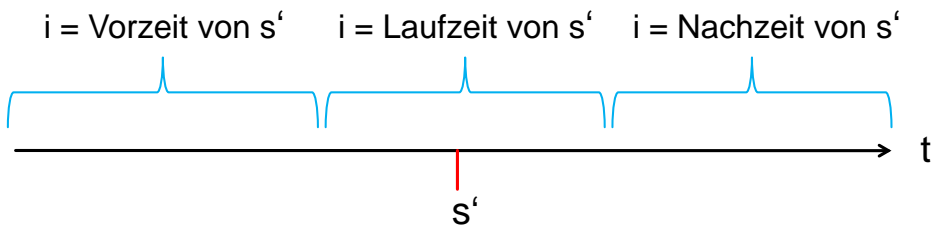

Abbildung 9.2.: Die drei Intervalle der temporalen Nachbarschaft

Wie bereits mehrfach angedeutet, besteht der semantische Beitrag eines Temporalsatzes nach Johnston (1994) in der Denotation eines Intervalls. Genauer gesagt, beschreibt ein Temporalsatz in Abhängigkeit von der ihn einleitenden Subjunktion eines der drei in Abbildung 9.2 dargestellten Intervalle. Je nachdem, welches der drei Intervalle denotiert wird, können temporale Subjunktionen in drei Untergruppen unterteilt werden. Die wichtigsten temporalen Subjunktionen sind in der folgenden, nicht-exhaustiven Einteilung eingetragen. ${ }^{65}$

\section{Einteilung der temporalen Subjunktionen nach der Art ihres De- notats}

(i) Als, während, (so)wie, solange, sobald, da und sooft bilden die Laufzeit.

(ii) Bevor, bis und ehe bilden die Vorzeit.

(iii) Nachdem, kaum dass, seit(dem), sobald, sowie und $d a$ bilden die Nachzeit.

Die Intervalle beziehen sich auf die im Nebensatz ausgedrückte Ereignisbeschreibung. Beispielsweise beschreibt der komplexe Ausdruck bevor (Jap einen Kuchen backte) die Vorzeit des Kuchenbackens, d.h. das Zeitintervall, das mit dem Moment endet, in dem Jap anfängt, den Kuchen zu backen. So kommt es zu einer terminologischen Umkehrung der traditionellen Begriffe Vor- und Nachzeitigkeit, welche sich in relationalen Ansätzen an dem Hauptsatz orientieren. Solche Subjunktionen, von denen klassischerweise angenommen wird, dass sie eine zweistellige Relation der

\footnotetext{
${ }^{64}$ Johnston (1994) bezeichnet diese drei Intervalle als runtime, foremath und aftermath.

65 Zur Klassifikation der temporalen Subjunktionen nach den Konzepten Gleichzeitigkeit, Vorzeitgkeit und Nachzeitigkeit vgl. u.a. Buscha (1989), Eisenberg (1989), Eisenberg et al. (2009), Pasch et al. (2003) und Zifonun et al. (1997).
} 
Gleichzeitigkeit herstellen, denotieren im Rahmen der hier vorgestellten Intervallanalyse die Laufzeit der im Adverbialsatz ausgedrückten Eventualität. Analog bilden die Subjunktionen, die in einer relationalen Analyse Nachzeitigkeit herstellen, das Intervall Vorzeit und die Subjunktionen, die klassischerweise eine zweistellige Relation der Vorzeitigkeit herstellen, denotieren die Nachzeit. Eine Gegenüberstellung der beiden Konzepte liefert Abbildung 9.9 am Ende dieses Kapitels.

Der hier vorgeschlagene Ansatz, demzufolge Temporalsätze ein Zeitintervall denotieren, zeigt große Ähnlichkeiten mit der Analyse des semantischen Beitrags von Präpositionen in der IDS-Grammatik (vgl. Zifonun et al. 1997, 2098ff). Auch bei temporalen Präpositionen gewinnt man den Eindruck, dass es sich um relationale Ausdrücke handelt, aus diesem Grund werden Präpositionen im Deutschen ja auch als Verhältniswörter bezeichnet. Dennoch handelt es sich bei Präpositionen weder syntaktisch noch semantisch um zweistellige Ausdrücke. Zifonun et al. (1997, 2098ff) gehen davon aus, dass Präpositionen über eine Binnen- und eine Außensemantik verfügen. Die binnensemantische Eigenschaft von Präpositionen besteht darin, ein sogenanntes inneres Argument, typischerweise eine NP, zu fordern und diese auf ein Denotat abzubilden. Beispielsweise kann die Präposition auf als Argument die NP dem Tisch nehmen und als Denotat eine Orts- oder Regionsbeschreibung liefern. Zifonun et al. $(1997,2099)$ bezeichnen dieses Denotat als die „AUF-Region des Tisches". Analog dazu lässt sich feststellen, dass eine temporale Präposition wie nach in einer PP wie nach der Feier die definite NP die Feier als inneres Argument nimmt und sie auf ein Zeitintervall, nämlich die „NACH-Zeitspanne der Feier“, abbildet. Temporale Subjunktionen funktionieren ganz ähnlich, mit dem Unterschied, dass sie ein IP- anstatt eines NP-Komplements fordern, um es auf ein Zeitintervall i abzubilden. Die entsprechende syntaktische Struktur ist in (336) abgebildet:

$$
\text { [CP während [IP Evi auf den Bus wartete] }]
$$

Präpositionen stiften aber auch eine Relation zu einem äußeren Argument, was ihre Außensemantik ausmacht. Dieses äußere Argument wird im Falle von auf durch eine Lokalisierungsrelation charakterisiert, wie beispielsweise in dem folgenden Satz aus Zifonun et al. (1997, 2100):

$$
\text { Das Buch ist auf der Bank. }
$$

Das äußere Argument Buch wird bezogen auf das Denotat der PP, also die Ortsbeschreibung oder "AUF-Region der Bank", räumlich verortet. Wesentlich ist jedoch, dass das äußere Argument kein Komplement der Präposition ist (vgl. Zifonun et al. 1997, 2100). Dasselbe gilt für die temporale Beziehung in Beispiel (338), mit dem Unterschied, dass hier eine Relation zwischen Ereignisbeschreibungen anstatt zwischen Gegenständen vorliegt.

$$
\text { Während der Pause/Nach dem Mittagessen war Evi gut gelaunt. }
$$

Die Ereignisbeschreibung Evi war gut gelaunt bildet das äußere Argument der einstelligen Präposition nach und wird bezogen auf die Intervallbeschreibung nach (dem 
Mittagessen) zeitlich verortet. Die temporale Relation zwischen zwei Ereignisbeschreibungen ist demnach auch im Rahmen einer einstelligen Analyse von temporalen Präpositionen ableitbar. Der wesentliche Unterschied liegt dabei in der Art der kompositionalen Herleitung. Bevor wir den außensemantischen Beitrag von temporalen Subjunktionen betrachten, soll ihre Binnensemantik jedoch noch etwas genauer untersucht werden.

Durch die Wahl der temporalen Subjunktion wird spezifiziert, ob es sich bei dem Denotat um die Lauf-, die Vor- oder die Nachzeit der in IP beschriebenen Situation handelt. In Anlehnung an die semantische Beschreibung von lokalen Präpositionen in Zifonun et al. (1997, 2098ff), derzufolge lokale Präpositionen regionenkonstituierend sind, wird dieser Bedeutungsaspekt von temporalen Subjunktionen im Folgenden als intervallkonstituierend bezeichnet.

Subjunktionen, die wie während in Gruppe (i) der drei in (335) klassifizierten Untergruppen gehören, bilden nach Johnston (1994) zusammen mit ihrem Argument die Laufzeit der jeweiligen Situationsbeschreibung. Bezogen auf den Beispielsatz in (336) sieht das folgendermaßen aus: Evi auf den Bus wartete bildet eine Situationsbeschreibung, abgekürzt als s. Als solche kann sie Komplement der temporalen Subjunktion während sein. Angewandt auf s, liefert während die Laufzeit von s, also die maximale Zeitspanne, die s auf einem Zeitstrahl einnimmt. Als Laufzeitfunktion wird ab sofort die Abkürzung $\tau$ verwendet.

$$
\begin{aligned}
& \text { während }(\mathrm{s})=\mathrm{i}, \\
& \text { wobei } \mathrm{i}=\text { Laufzeit von s }(=\tau(\mathrm{s}))
\end{aligned}
$$

Die Denotation des Adverbialsatzes (336) lautet demnach $\mathrm{i}=\tau(\mathrm{s})$, wobei i der maximalen Zeitspanne entspricht, in der die durch Evi bezeichnete Person auf den Bus wartet. Dies ist in Grafik 9.3 veranschaulicht.

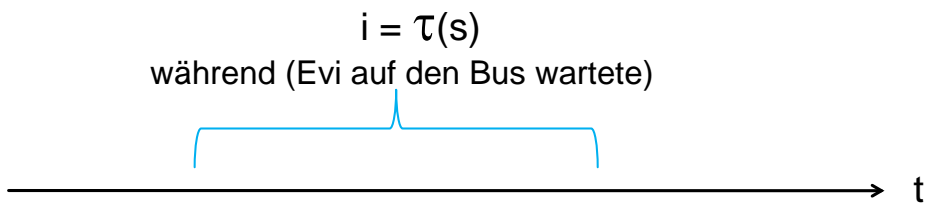

Abbildung 9.3.: Denotation eines während-Satzes

Neben als und während drücken noch weitere Subjunktionen die Laufzeit aus. Dazu gehören wie, solange, sowie, sooft, temporal verwendetes $d a$ und bestimmte Verwendungen von sobald (zu den semantischen Unterschieden zwischen den einzelnen Subjunktionen vgl. u.a. Buscha 1989, 452, Eisenberg 1989, Eisenberg et al. 2009, 627 und Pasch et al. 2003). Auch in dem folgenden Beispiel bezeichnet der Adverbialsatz die Laufzeit der Situation Christel wachte auf. Da es sich um ein punktuelles Prädikat handelt, besteht das entsprechende Intervall, definiert als eine Menge von 
Zeitpunkten, nur aus einem einzigen Zeitpunkt.

$$
\text { Als Christel heute Morgen aufwachte, war es noch dunkel. }
$$

Betrachten wir nun den binnensemantischen Beitrag von bevor als Beispiel für eine Subjunktion, deren Funktion klassischerweise als „Ausdruck der Nachzeitigkeit“ betrachtet wird. Dabei werden kontrafaktische Interpretationen ausgeklammert. Wie während nimmt auch bevor eine Situationsbeschreibung s als Argument und bildet sie auf ein Intervall ab. In diesem Fall handelt es sich jedoch nicht um die Laufzeit von s, sondern um die Vorzeit. Als Funktion, welche aus einer Situationsbeschreibung deren Vorzeit liefert, wird der Operator $\xi$ verwendet.

$$
\begin{aligned}
& \operatorname{bevor}(\mathrm{s})=\mathrm{i} \\
& \text { wobei } \mathrm{i}=\text { Vorzeit von } \mathrm{s}(=\xi(\mathrm{s}))
\end{aligned}
$$

Die Denotation des Adverbialsatzes bevor Evi auf den Bus wartete ist in Grafik 9.4 dargestellt. Das entsprechende Intervall umfasst die Zeitspanne, welche vor Beginn des Wartens liegt.

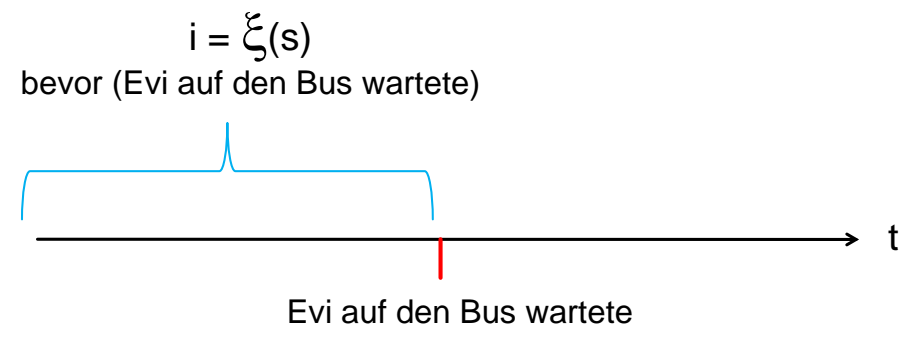

Abbildung 9.4.: Denotation eines bevor-Satzes

Schließlich soll zur Vervollständigung noch der Beitrag der Subjunkion nachdem als Beispiel für Gruppe (iii) (vgl. (335)) betrachtet werden. Johnston (1994) argumentiert, dass das englische after sein Komplement auf die Nachzeit der jeweiligen Situationsbeschreibung abbildet. Das Gleiche gilt auch für die deutsche Subjunktion nachdem, die zusammen mit ihrem IP-Argument die Nachzeit einer Situationsbeschreibung denotiert. In dem folgenden Beispiel bezeichnet der Adverbialsatz demnach das Intervall i, für das gilt, dass es direkt nach Ende der im Adverbialsatz ausgedrückten Situation beginnt.

(342) (Barbara kochte Nudeln,) nachdem Mailin angerufen hatte.

Wie bei den temporalen Subjunktionen der Gruppen (i) und (ii) handelt es sich auch bei nachdem um einen einstelligen Ausdruck. Für die Funktion, welche die Nachzeit einer Situation ausdrückt, wird das Zeichen $\nu$ verwendet.

$$
\operatorname{nachdem}(\mathrm{s})=\mathrm{i},
$$


wobei $\mathrm{i}=$ Nachzeit von $\mathrm{s}(=\nu(\mathrm{s}))$

Das Denotat des Adverbialsatzes aus Beispiel (342) ist in Abbildung 9.5 zu sehen. Der rechte Rand des Intervalls ist durch die Sprechzeit (S) begrenzt, da die Verwendung des Plusquamperfekts im Adverbialsatz sowie die einfache Vergangenheit im Hauptsatz anzeigen, dass beide Ereignisse vor der Sprechzeit liegen.

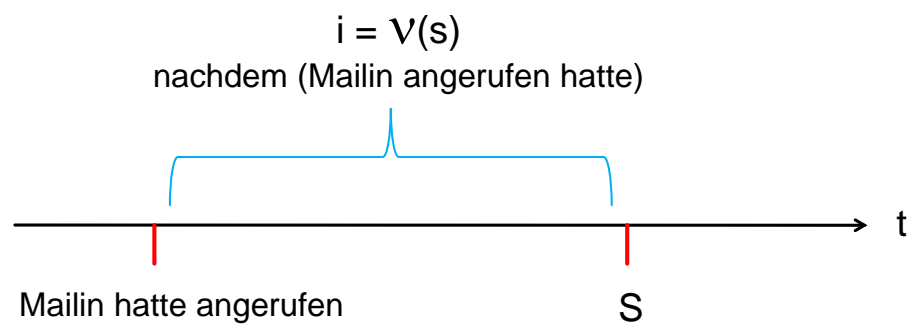

Abbildung 9.5.: Denotation eines nachdem-Satzes

Als Zwischenergebnis können wir festhalten, dass der binnensemantische Aspekt von temporalen Subjunktionen darin besteht, die von ihnen eingeleitete Proposition auf ein Intervall i abzubilden. Im Rahmen dieser Analyse verhalten sich temporale Subjunktionen demnach genauso wie Präpositionen bei Zifonun et al. (1997, 2098ff). Anstatt eine Relation zwischen zwei Komplementen auszudrücken, handelt es sich um einstellige Operatoren, die eine Intervallbeschreibung liefern.

Auch wenn es sich bei temporalen Subjunktionen um einstellige Ausdrücke handelt, haben wir dennoch den Eindruck, dass Haupt- und Nebensatz in einer zeitlichen Relation zueinander stehen. Auch in diesem Punkt gleichen sie Präpositionen, welche nach Zifonun et al. (1997, 2098ff) eine Relation zu einem „äußeren“ Argument stiften, bei dem es sich jedoch um kein Komplement der einstelligen Präpositionen handelt. So wird in Beispiel (337), hier wiederholt als (344), eine Lokalisierungsrelation zwischen zwei Gegenständen ausgedrückt. Dabei wird das äußere Argument Buch bezogen auf das Denotat der PP, also die Ortsbeschreibung oder „AUF-Region der Bank", räumlich verortet.

(344) Das Buch ist auf der Bank.

Zentral ist, dass dieses äußere Argument kein Komplement der Präposition ist. Ganz ähnlich funktioniert nun die temporale Beziehung zwischen Haupt- und temporalem Adverbialsatz. Letzterer denotiert ein Intervall, bezogen auf das der Hauptsatz, also das äußere Argument, interpretiert wird. Dieser Bedeutungsaspekt von temporalen Subjunktionen soll in Anlehnung an die Analyse von PPn in Zifonun et al. (1997, 2098ff) als relationsstiftend bezeichnet werden. Betrachten wir dies etwas genauer.

Nach Johnston (1994) kommt der relationsstiftende Aspekt von Temporalsätzen dadurch zustande, dass Temporalsätze als Quantorrestriktionen fungieren, wie wir in 
Abschnitt 8.2 gesehen haben. Aus diesem Grund kann aus dem komplexen Satz (345), in dem der Hauptsatz im Nukleus eines Allquantors steht, nicht geschlossen werden, dass Evi immer Brezeln knabbert. Die beiden Argumente des Allquantors, Restriktion und Nukleus, werden hier durch den Neben- und den Hauptsatz realisiert.

$$
\text { Evi knabbert immer eine Brezel, während sie fernsieht. }
$$

Die Subjunktion während bildet zusammen mit ihrem Komplement $\mathrm{s}=$ Evi sieht fern die Laufzeit von s, also $\mathrm{i}=\tau(\mathrm{s})$. Zentral ist nun, dass durch dieses Intervall die Domäne des Allquantors auf solche Zeitspannen restringiert wird, für die gilt, dass Evi in ihnen fernsieht. Der Hauptsatz wird in einem zweiten Schritt bezogen auf diese Intervalle interpretiert, wodurch die temporale Relation zwischen Haupt- und Nebensatz entsteht. Genauer gesagt liegt eine mengentheoretische Relation zwischen zwei Intervallen vor, für die, wie in (346) dargestellt, gilt, dass das Hauptsatzereignis e innerhalb des Intervalls i stattgefunden haben muss. Die Abkürzung f bezeichnet dabei eine Funktion zur Bildung der Laufzeit des Hauptsatzereignisses. (346) ist zu lesen als „für jedes Interval i, in dem Evi fernsieht, muss es ein Ereignis e geben, auf das die Beschreibung, dass Evi eine Brezel knabbert, zutrifft, und dessen Laufzeit $\mathrm{f}(\mathrm{e})$ in dem Interval i enthalten ist". Dies ist in Grafik 9.6 bildlich veranschaulicht.

$$
\forall\{\mathrm{i}=\tau(\mathrm{s})\}[\mathrm{e} \wedge \mathrm{f}(\mathrm{e}) \subseteq \mathrm{i}]
$$

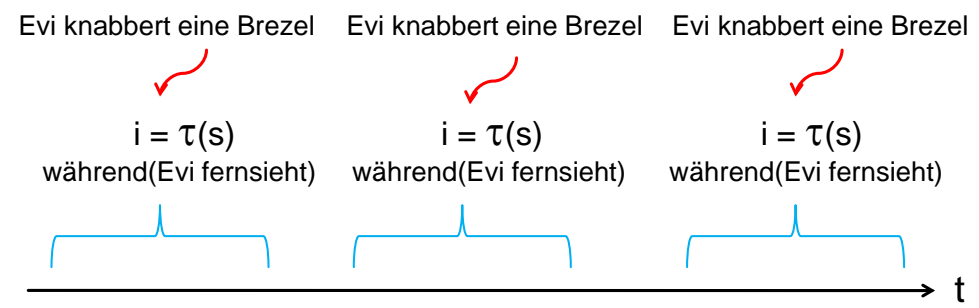

Abbildung 9.6.: Restriktion eines Allquantors durch einen während-Satz

Der relationsstiftende Bedeutungsaspekt von temporalen Subjunktionen ist demnach nur anscheinend der Einführung einer zweistelligen Relation zu verdanken. Stattdessen zeigt Johnston (1994) für das Englische, und wir haben in Abschnit 8.2 gesehen, dass die gleiche Beobachtung auch für das Deutsche gemacht werden kann, dass das durch einen Temporalsatz beschriebene Zeitintervall wie in Beispiel (345) einen Quantor restringiert, in dessen Nukleus der Hauptsatz steht (vgl. dazu auch de Swart 1991). Da die Domäne des Quantors nur auf die durch den Temporalsatz bezeichneten Intervalle beschränkt wird, wird der Hauptsatz auch nur in Bezug auf diese relevanten Zeitspannen interpretiert. Dadurch werden Haupt- und Nebensatz 
temporal zueinander in Beziehung gesetzt.

Im Zusammenhang mit während wird der Allquantor als quantifizierendes Adverb realisiert. Dagegen enthält die Subjunktion sooft bereits den Allquantor. Aus diesem Grund ist Abbildung 9.6 auch eine korrekte Wiedergabe des folgenden Satzes:

Evi knabbert eine Brezel, sooft sie fernsieht.

Wichtig ist nun, dass Temporalsätze, beziehungsweise die durch sie ausgedrückten Intervalle, laut de Swart (1991) und Johnston (1994) genau wie Konditionalsätze in der Analyse von Kratzer (1991) und Lewis (1975) immer als Quantorrestriktionen fungieren. Ist wie in Beispiel (348-a) kein overter Quantor vorhanden, muss von der Existenz eines koverten Quantors ausgegangen werden, so dass (348-a) eine logische Struktur wie in (348-b) erhält. Auch hier wird demnach die Domäne des Quantors, in dessen Nukleus der Hauptsatz steht, auf das Intervall $\tau(\mathrm{s})$ restringiert. Die entsprechende Interpretation ist in Abbildung 9.7 dargestellt.

a. Evi knabberte eine Brezel, während sie fernsah.

b. $\exists\{\mathrm{i}=\tau(\mathrm{s})\}[\mathrm{e} \wedge \mathrm{f}(\mathrm{e}) \subseteq \mathrm{i}]$

Evi knabbert eine Brezel

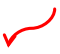

$\mathrm{i}=\tau(\mathrm{s})$

während(Evi fernsieht)

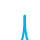

Abbildung 9.7.: Restriktion eines (koverten) Existenzquantors durch einen während-Satz

Ein Nachteil der hier vorgestellten Analyse von temporalen Subjunktionen besteht folglich darin, dass die Annahme eines koverten Existenzquantors notwendig ist. Da die Daten in Kapitel 8 jedoch zeigen, dass sich temporale Subjunktionen nicht wie zweistellige Ausdrücke verhalten, erscheint diese Annahme gerechtfertigt und nicht stipulativ.

Die temporale Situierung des Hauptsatzereignisses bezogen auf das im Nebensatz ausgedrückte Intervall hängt davon ab, ob der Hauptsatz telisch oder atelisch ist. Drückt der Hauptsatz wie in den Beispielen (345) und (348-a) ein telisches Ereignis, also ein Ereignis mit einem natürlichen Endpunkt, aus, muss die Dauer des Hauptsatzereignisses in i enthalten oder gleich i sein. Bezogen auf Beispiel (348-a) bedeutet dies, dass Evi für den Verzehr der Brezel maximal die Zeitspanne, in der sie fernsah, benötigt hat. Diese mengentheoretische Relation wird dagegen umgekehrt, wenn der Hauptsatz ein atelisches Ereignis, also ein Ereignis ohne natürlichen Endpunkt, ausdrückt wie in dem folgenden Beispiel: 
Jap war im Garten, während Evi fernsah.

In diesem Fall muss das durch den Adverbialsatz bezeichnete Intervall in der Laufzeit des Hauptsatzereignisses enthalten sein. Dabei können die beiden Intervalle deckungsgleich sein, was bedeutet, dass sich Jap die gesamte Zeit, die Evi ferngesehen hat, im Garten aufhielt, aber nicht davor und auch nicht danach. Das Hauptsatzereignis kann aber auch länger andauern als das durch den Temporalsatz denotierte Intervall. In diesem Fall war Jap beispielsweise schon im Garten, bevor Evi fernsah, oder er blieb danach noch länger dort. Wesentlich ist jedoch, dass er die gesamte Zeit, die Evi fernsah, im Garten verbracht hat. Die Interpretation des Hauptsatzes bezogen auf den Temporalsatz in Abhängigkeit der lexikalischen Aktionsart ist in den Wahrheitsbedingungen in (350) nach Johnston $(1994,96)$ zusammengefasst. Die Funktion g definiert dabei, ob eine Ereignisbeschreibung $\Psi$ bezogen auf i wahr oder falsch ist.

a. Wenn $\Psi$ telisch ist, dann gilt: $\mathrm{g}_{\Psi}(\mathrm{i})=1$, wenn $\exists$ e $[\Psi(\mathrm{e}) \&[\mathrm{f}(\mathrm{e}) \subseteq \mathrm{i}]]$ b. Wenn $\Psi$ atelisch ist, dann gilt: $\mathrm{g}_{\Psi}(\mathrm{i})=1$, wenn $\exists$ e $[\Psi(\mathrm{e}) \&[\mathrm{f}(\mathrm{e}) \supseteq \mathrm{i}]]$

Fassen wir den Beitrag von temporalen Subjunktionen zur zeitlichen Einordnung des jeweiligen Matrixsatzes kurz zusammen. Als einstellige Operatoren stellen temporale Subjunktionen keine Relation zwischen zwei Komplementen her, der Hauptsatz besetzt also keine semantische Leerstelle der Subjunktion. Vielmehr bilden Hauptund temporaler Nebensatz die zwei Argumente eines overten oder koverten Quantors. Das durch den Temporalsatz denotierte Intervall restringiert die Domäne dieses Quantors, in dessen Nukleus der Hauptsatz steht. Dieser wird dann nur in Bezug auf die durch den Temporalsatz beschriebenen Intervalle interpretiert. Dadurch ergibt sich die zeitliche Beziehung zwischen Haupt- und Nebensatz indirekt über den sie verbindenden Quantor, ohne dass eine spezielle Festlegung der Relation in der lexikalischen Semantik der einzelnen Subjunktionen notwendig wäre. In diesem Punkt unterscheidet sich die hier vorgestellte Analyse von der Beschreibung des semantischen Beitrags von Präpositionen in Zifonun et al. (1997), in welcher davon ausgegangen wird, dass der relationsstiftende Beitrag in der lexikalischen Semantik der Präpositionen verankert ist. Am Beispiel der Präposition auf argumentieren Zifonun et al. (1997, 2100), dass die Präposition nicht nur die „AUF-Region“ ihres inneren Arguments y bezeichnet, sondern mehr noch ,in der AUF-Region von y befindlich“ bedeutet. Bezogen auf temporale Subjunktionen ist eine solche Annahme nicht notwendig, da der relationsstiftende Aspekt aus ihrer Funktion als Quantorrestriktionen abgeleitet werden kann. ${ }^{66}$

${ }^{66}$ Möglicherweise kommt auch der relationsstiftende Aspekt von temporalen Präpositionen über ihre Funktion als Restriktion eines Quantors zustande. Dass die Domäne eines Quantors durch eine temporale, nicht aber durch eine kausale Präposition restringiert wird, belegen die folgenden Beispiele. So folgt (i-c) zwar aus (i-b), nicht aber aus (i-a).

(i) a. Vor dem Frühstück ist Martin immer schlecht gelaunt.

b. Wegen seines Jobs ist Martin immer schlecht gelaunt. 
Was hier bezogen auf den außensemantischen Beitrag von während dargestellt wurde, trifft im Wesentlichen auch auf die Subjunktionen der Gruppen (ii) und (iii) zu. Dies soll hier kurz am Beispiel der Subjunktion bevor gezeigt werden. In Kapitel 8.2 haben wir gesehen, dass auch mit bevor oder nachdem eingeleitete Sätze in quantifizierten Strukturen die Rolle der Restriktion übernehmen (vgl. die Beispiele (325) und (326)). Wie der während-Satz in (345) oder (348-a) beschränken demnach alle Temporalsätze die Domäne eines overten oder koverten Quantors auf die durch sie denotierten Intervalle. Der Hauptsatz wird dann nur in Bezug auf diese Intervalle interpretiert (vgl. dazu (346), (348-b) sowie die Abbildungen 9.6 und 9.7). Der einzige Unterschied zwischen den Subjunktionen der drei in (335) dargestellten Gruppen besteht in der Art des durch sie beschriebenen Intervalls. Wurde der Hauptsatz in Beispiel (348-a) bezogen auf die Laufzeit der Adverbialsatzsituation interpretiert, so muss beispielsweise der Hauptsatz in (351) bezogen auf die Vorzeit der im Adverbialsatz ausgedrückten Situation ausgewertet werden.

$$
\text { Barbara strickte einen Schal, bevor sie ins Büro fuhr. }
$$

Da der Hauptsatz telisch ist, muss das in ihm ausgedrückte Ereignis im Intervall $\xi(\mathrm{s})$, also der Zeit, die vor dem Gang ins Büro liegt, abgeschlossen sein. Beispiel (351) drückt also aus, dass das Stricken des Schals beendet ist, bevor die im Adverbialsatz ausgedrückte Situation beginnt. Die Interpretation des Hauptsatzes bezogen auf das durch den Temporalsatz denotierte Intervall ist in Abbildung 9.8 dargestellt.

Ist der Haupsatz atelisch wie in Beispiel (352), muss lediglich der Beginn des im Hauptsatz ausgedrückten Ereignisses innerhalb von $\xi(\mathrm{s})$ liegen, es kann aber auch noch weiter andauern.

$$
\text { Daniel lachte schon, bevor Jenny den Witz zu Ende erzählt hatte. }
$$

Es handelt sich dabei um dieselben Wahrheitsbedingungen, wie wir sie bereits in (350) für während kennengelernt haben (vgl. dazu auch Heinämäki 1978). Der se-

c. Martin ist immer schlecht gelaunt.

Die Beispiele legen eine Analyse analog zu der, die bezogen auf temporale Subjunktionen vorgeschlagen wurde, nahe. Anstatt den relationsstiftenden Aspekt von temporalen Subjunktionen in ihrer lexikalischen Semantik festzulegen, könnte davon ausgegangen werden, dass eine temporale PP die Domäne eines Quantors auf die von ihr denotierten Intervalle beschränkt. Das im Satz ausgedrückte Ereignis, in diesem Fall Martins schlechte Laune, muss dann in diesen Intervallen enthalten sein. Ist kein overter Quantor gegeben, muss von einem koverten ausgegangen werden. Diese Analyse hätte den Vorteil, dass es sich bei temporalen Präpositionen auch semantisch tatsächlich um einstellige Ausdrücke handeln würde. Dadurch, dass das durch sie gebildete Intervall aber selbst wiederum Argument eines Quantors ist, kann die Relation zum Restsatz zustande kommen. Im Gegensatz dazu bleibt bei Zifonun et al. (1997, 2098ff) offen, auf welche Weise ein eigentlich einstelliger Ausdruck eine Relation zu einem „äußeren“ Argument herstellen kann und wodurch sich dieses äußere Argument von einem tatsächlichen Komplement unterscheidet. Auch der Duden (2009, 600ff) lässt diesbezüglich Fragen offen. Zum einen werden Präpositionen als Bindewörter bezeichnet, die zwei Größen zueinander in Bezug setzen, gleichzeitig wird aber postuliert, dass Präpositionen nur eine Ergänzung fordern. 


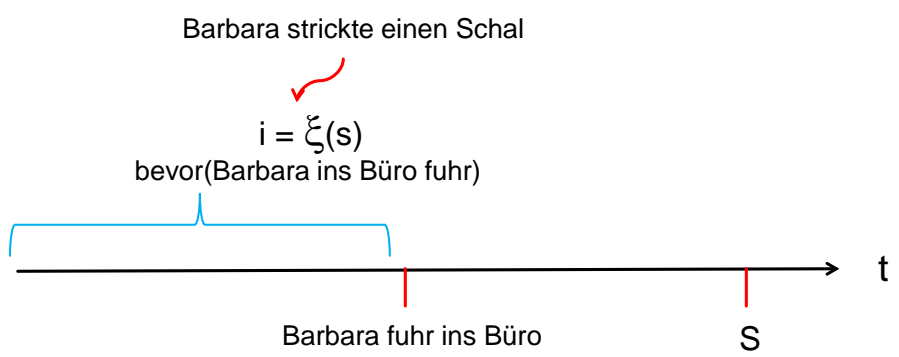

Abbildung 9.8.: Restriktion eines (koverten) Existenzquantors durch einen bevor-Satz

mantische Unterschied zwischen bevor und während besteht demnach nicht darin, dass der Hauptsatz unterschiedlich in Bezug auf i eingeordnet wird. Vielmehr unterscheiden sich die beiden Gruppen von Subjunktionen in der Art des Intervalls, das sie abbilden: Laufzeit im Falle von während und Vorzeit im Falle von bevor. Die beiden Gruppen von Subjunktionen fokussieren demnach unterschiedliche Komponenten der in Abbildung 9.2 dargestellten temporalen Nachbarschaft.

Zusammengefasst können wir festhalten, dass temporale Subjunktionen keine zweistellige Relation zwischen zwei Sätzen herstellen. Stattdessen denotieren sie ein Zeitintervall, indem sie in Form der von ihnen eingeleiteten IP eine Situationsbeschreibung als Argument nehmen. Nach Johnston (1994) kann sich dieses Intervall auf eine von drei verschiedenen zeitlichen Komponenten der im Adverbialsatz ausgedrückten Proposition beziehen: Ihre Laufzeit, ihre Vorzeit, also das vor Beginn ihrer Laufzeit liegende Intervall, oder die nach Ende ihrer Laufzeit liegende Zeitspanne der Nachzeit (vgl. Abbildung 9.2). Das durch einen Temporalsatz denotierte Intervall restringiert zudem immer die Domäne eines overten oder koverten Quantors, in dessen Nukleus der Hauptsatz steht. Dadurch wird der Hauptsatz nur bezogen auf die durch den Temporalsatz beschriebenen Zeitspannen interpretiert.

Im Unterschied zu Ansätzen wie Buscha (1989), Eisenberg (1989), Eisenberg et al. (2009), Heinämäki (1978), Helbig/Buscha (1986), Pasch et al. (2003) und de Swart (1991), welche davon ausgehen, dass temporale Subjunktionen eine zweistellige Relation einführen, ergibt sich die zeitliche Relation zwischen Haupt- und Nebensatz in dieser Analyse nach Johnston (1994) erst in einem zweiten Schritt durch die Einschränkung des Quantors auf bestimmte Zeitintervalle. Eine Gegenüberstellung der beiden unterschiedlichen Ansätze bietet Abbildung 9.9.

Im Rahmen der in diesem Abschnitt vorgestellten Analyse besitzen eine temporale $\mathrm{PP}$ wie vor der Landung und der Temporalsatz bevor das Flugzeug landete dasselbe Denotat. In beiden Fällen bildet ein einstelliger Operator in $\mathrm{P}^{0}$ bzw. in $\mathrm{C}^{0}$ sein Komplement auf ein Zeitintervall ab, und zwar die Vorzeit der Landung des Flugzeugs. Beide Formen von Zeitausdrücken erfüllen im Rahmen einer Reichenbach'schen Tempustheorie (vgl. Reichenbach 1947) dieselbe Funktion, indem sie die Referenzzeit R ausdrücken. Beispielsweise muss das im Hauptsatz ausgedrückte Ereignis in der folgenden Äußerung zeitlich in der Vergangenheit vor einem Referenzzeitpunkt, der 


\begin{tabular}{|c|c|c|}
\hline & $\begin{array}{l}\text { Temporale Subjunktionen } \\
\text { sind zweistellig: Relation } \\
\text { zwischen zwei Zeitpunkten t }\end{array}$ & $\begin{array}{l}\text { Temporale Subjunktionen } \\
\text { sind einstellig: Denotation } \\
\text { eines Intervalls i }\end{array}$ \\
\hline $\begin{array}{l}\text { als, während, } \\
\text { (so)wie, } \\
\text { solange, } \\
\text { sobald, da, } \\
\text { sooft }\end{array}$ & $\begin{array}{l}\text { Gleichzeitigkeit } \\
\begin{array}{r}\mathrm{t}_{\mathrm{HS}} \subseteq I \supseteq \mathrm{t}_{\mathrm{NS}} \\
\mathrm{HS}, \mathrm{NS} \\
\end{array}\end{array}$ & $\underset{\mathrm{i}}{\mathrm{i}(\mathrm{HS}) \subseteq I \supseteq \mathrm{i}}$ \\
\hline $\begin{array}{l}\text { bevor, bis, } \\
\text { ehe }\end{array}$ & $\begin{array}{c}\text { Nachzeitigkeit } \\
\mathrm{t}_{\mathrm{HS}}<\mathrm{t}_{\mathrm{NS}} \\
\mathrm{HS} \\
\end{array}$ & $\overbrace{14444 H} \begin{array}{l}\mathrm{i}=\text { Vorzeit } \\
\mathrm{HS}(\mathrm{HS}) \subseteq I \supseteq \mathrm{i}\end{array}$ \\
\hline $\begin{array}{l}\text { nachdem, kaum } \\
\text { dass, seit(dem), } \\
\text { sobald, sowie, } \\
\text { da }\end{array}$ & $\begin{array}{c}\text { Vorzeitigkeit } \\
\mathrm{t}_{\mathrm{NS}}<\mathrm{t}_{\mathrm{HS}} \\
\quad \mathrm{NS} \\
\end{array}$ & 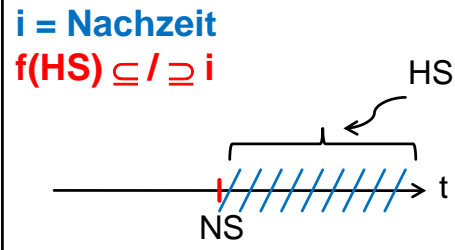 \\
\hline
\end{tabular}

Abbildung 9.9.: Gegenüberstellung der ein- und zweistelligen Analyse von temporalen Subjunktionen

ebenfalls in der Vergangenheit liegt, stattgefunden haben. Die Referenzzeit wird in diesem Fall durch den Temporalsatz bzw. die PP geliefert (vgl. dazu auch Blühdorn 2004b, 128, Blühdorn 2004a, Hinrichs 1986, Kamp/Reyle 1993, Lohnstein 1996, 211, Partee 1984, Roberts 1995, 663 und Rothstein 2007, 72). ${ }^{67}$

Als Spiros in den Garten kam/Um 12 Uhr, hatte Kostas die Pflanzen schon bewässert.

Aus dieser Annahme, derzufolge temporale PPn und Adverbialsätze funktional äquivalent sind, ergibt sich die Frage, welche Faktoren die Wahl einer der beiden Formen beeinflussen. Da eine CP mehr semantischen Inhalt besitzt als eine PP, kann ein Temporalsatz besser akkommodiert werden und ist demnach besser geeignet in Kontexten, in denen das Komplement neu und nicht vorerwähnt ist. Auf diesen Punkt wird in Abschnitt 9.1.3 noch genauer eingegangen. Umgekehrt erlaubt die Verwendung einer PP den Wegfall des Subjekts der Handlung, zudem ist eine PP nicht nach Tempus markiert, was in bestimmten Kontexten von Vorteil sein kann.

\footnotetext{
${ }^{67}$ Laut Roberts (1995, 663) fungiert das Reichenbach'sche R zudem als Restriktor in quantifizierten Strukturen, indem es über Intervalle quantifiziert. Diese Ansicht deckt sich mit den in Kapitel 8.2 gewonnenen Ergebnissen und stützt die Annahme, dass Temporalsätze, die immer Quantorrestriktoren sind, den Parameter $\mathrm{R}$ realisieren.
} 


\subsubsection{Verortung des Hauptsatzes innerhalb des Intervalls}

Ein Aspekt bleibt in der von Johnston (1994) vorgeschlagenen Analyse unberücksichtigt. Mitunter ist das durch einen Temporalsatz denotierte Intervall sehr bis unendlich lang. Beispielsweise bezeichnet der Nebensatz in dem folgenden Beispiel ein Intervall, welches sich so lange in die Vergangenheit erstreckt, bis es auf eine Situation mit derselben Beschreibung wie der des Nebensatzes stößt. Das entsprechende Intervall kann demnach Tage, Wochen oder Jahre umfassen und es ist in seinem linken Rand sogar komplett unbegrenzt, falls die durch Maria bezeichnete Person noch nie zuvor nach Tibet gereist ist.

Maria telefonierte mit Peter, bevor sie nach Tibet reiste.

Der Hauptsatz kann innerhalb dieses Intervalls nun unterschiedlich positioniert werden, was in Abbildung 9.10 exemplarisch anhand der drei Pfeile dargestellt ist. Obwohl alle drei in Abbildung 9.10 gezeigten Interpretationen und noch viele weitere möglich sind, würde man den Satz typischerweise so verstehen, dass das Telefonat zumindest relativ kurz vor Marias Abfahrt stattgefunden hat und nicht etwa fünf Jahre vorher. Die unmarkierte Interpretation, derzufolge Maria und Peter wenige Stunden oder Tage vor Marias Reise telefoniert haben, ist in Abbildung 9.10 anhand des Pfeils Nummer 1 dargestellt. Eine Einordnung des Hauptsatzes an die durch Pfeil Nummer 3 bezeichnete Stelle im Intervall entspricht dagegen der markierten Lesart, derzufolge das Telefonat mehrere Jahre vor der Reise stattfand.

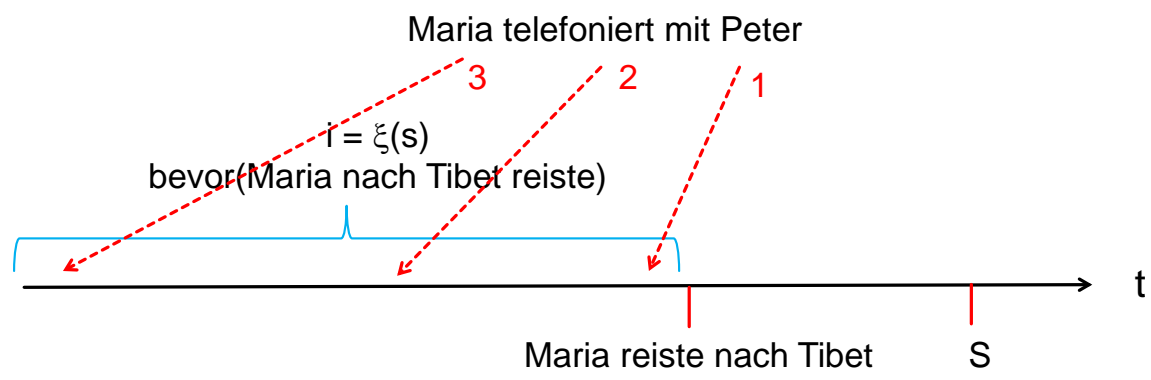

Abbildung 9.10.: Positionierung des Hauptsatzes innerhalb von i

Zu klären ist also, welche Faktoren, ob primär semantische oder pragmatische, darüber entscheiden, an welcher Stelle von i der Hauptsatz eingeordnet wird. Im Folgenden soll dafür argumentieren werden, dass es sich dabei um eine konversationelle Implikatur handelt. Genauer gesagt, ist B eine skalare Implikatur, die durch Äußerung A ausgelöst wird.

(355) A: Maria telefonierte mit Peter, bevor sie nach Tibet reiste.

B: Das Telefonat hat wenige Stunden/Tage vor der Reise stattgefunden. 
Wie andere skalare Implikaturen ist B auf Grundlage der Befolgung der 1. Submaxime der Quantitätsmaxime nach Grice (1989) rekonstruierbar („Mache deinen Beitrag so informativ wie erforderlich!“). Alles, was wir neben den Griceschen Maximen noch benötigen, ist das Konzept einer temporalen Skala verbunden mit dem Konzept der Informativität.

Auf einem Zeitstrahl können im Prinzip alle den Diskursteilnehmern bekannten Situationen verortet werden. Beispielsweise kann es Teil des Common Ground sein, dass Maria vor ihrer Tibetreise umgezogen ist $\left(=\mathrm{s}_{2}\right)$ und noch davor ihr Abitur gemacht hat $\left(=\mathrm{s}_{3}\right)$. Diese Situationen stehen nicht nur in einer temporalen Beziehung zueinander, sondern sie bilden auch Alternativen zur Besetzung der Komplementstelle von bevor. Anstatt der Vorzeit von $\mathrm{s}_{3}$ wie in Satz (354) kann auch die Vorzeit von $\mathrm{s}_{2}$ oder die von $\mathrm{s}_{3}$ gebildet werden, wodurch jeweils kleinere Intervalle entstehen, was in Abbildung 9.11 dargestellt ist.

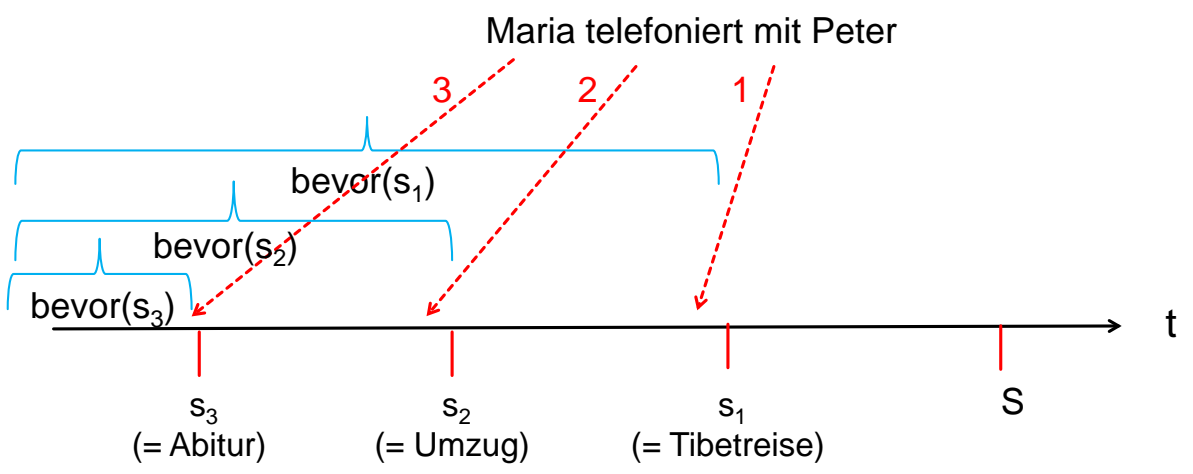

Abbildung 9.11.: Temporale Skala und Informativität

Entscheidend ist nun, dass sich diese Intervalle in ihrer Informativität unterscheiden. Dient ein Intervall der temporalen Verortung eines Ereignisses, ist es umso informativer, je kleiner es ist. Aus diesem Grund ist beispielsweise Satz (356-a) von größerer Informativität als Satz (356-b).

(356) a. Der Mord muss irgendwann zwischen 15 und 16 Uhr stattgefunden haben.

b. Der Mord muss irgendwann zwischen 15 und 20 Uhr stattgefunden haben.

Die in Abbildung 9.11 dargestellten Intervalle «bevor $\left(\mathrm{s}_{1}\right) \rrbracket(=$ bevor Maria nach Ti-

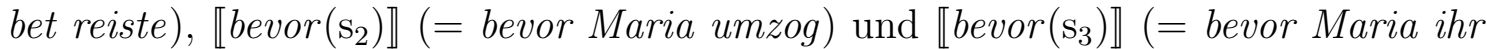
Abitur machte) bilden Ausdrucksalternativen, welche in einer Inklusionsbeziehung zueinander stehen. Es gilt:

$$
\llbracket \text { bevor }\left(\mathrm{s}_{3}\right) \rrbracket \subset \llbracket \text { bevor }\left(\mathrm{s}_{2}\right) \rrbracket \subset \llbracket \text { bevor }\left(\mathrm{s}_{1}\right) \rrbracket
$$


Damit ähneln die drei Intervalle skalaren Ausdrücken wie beispielsweise einige im Vergleich zu alle, welche eine Horn-Skala bilden (vgl. Horn 1972 und Horn 1984). Dölling (2010) definiert eine Horn-Skala folgendermaßen:

Eine Skala dieser Art ist ein geordnetes n-Tupel $<\alpha_{1}, \alpha_{2}, \alpha_{3} \ldots, \alpha_{n}>$, wobei gilt:

Wenn sich beliebige Sätze $\phi\left[\alpha_{1}\right], \phi\left[\alpha_{2}\right], \phi\left[\alpha_{3}\right]$ etc. nur im Vorkommen von $\alpha_{1}$ bzw. $\alpha_{2}$ bzw. $\alpha_{3}$ etc. unterscheiden, dann gilt:

$\phi\left[\alpha_{1}\right]$ ist informativer als $\phi\left[\alpha_{2}\right], \phi\left[\alpha_{2}\right]$ ist informativer als $\phi\left[\alpha_{3}\right]$ etc., d.h. es gilt: $\phi\left[\alpha_{1}\right] \rightarrow \phi\left[\alpha_{2}\right], \phi\left[\alpha_{2}\right] \rightarrow \phi\left[\alpha_{3}\right]$ etc., aber nicht umgekehrt.

Beispielsweise bilden die Ausdrücke < alle, die meisten, viele, einige, wenige $>$ eine Horn-Skala, wobei der Ausdruck am linken Ende der Skala am informativsten ist. Bei den Elementen einer Horn-Skala handelt es sich typischerweise um eine Menge an etablierten Ausdrucksalternativen. Bezogen auf unser Beispiel bildet auch die folgende Menge eine Horn-Skala:

$$
<\operatorname{bevor}\left(\mathrm{s}_{3}\right) \text {, bevor }\left(\mathrm{s}_{2}\right) \text {, bevor }\left(\mathrm{s}_{1}\right)>
$$

Ein Satz $\phi\left[\right.$ bevor $\left.\left(\mathrm{s}_{3}\right)\right]$ unterscheidet sich nur im Vorkommen von bevor $\left(\mathrm{s}_{3}\right)$ von einem Satz $\phi\left[\right.$ bevor $\left.\left(\mathrm{s}_{1}\right)\right]$.

$$
\phi\left[\text { bevor }\left(\mathrm{s}_{3}\right)\right] \text { : Maria telefonierte mit Peter, bevor sie ihr Abitur machte. }
$$
$\phi\left[\right.$ bevor $\left.\left(\mathrm{s}_{1}\right)\right]$ : Maria telefonierte mit Peter, bevor sie nach Tibet reiste.

$\phi\left[\right.$ bevor $\left.\left(\mathrm{s}_{3}\right)\right]$ ist informativer als $\phi\left[\right.$ bevor $\left.\left(\mathrm{s}_{1}\right)\right]$, da $\phi\left[\right.$ bevor $\left.\left(\mathrm{s}_{3}\right)\right] \rightarrow \phi\left[\right.$ bevor $\left.\left(\mathrm{s}_{1}\right)\right]$ gilt, nicht aber die umgekehrte Implikationsrichtung. Nehmen wir beispielsweise an, dass das Telefonat zum Zeitpunkt von $\mathrm{s}_{2}$ stattgefunden hat. Dann gilt zwar, dass es

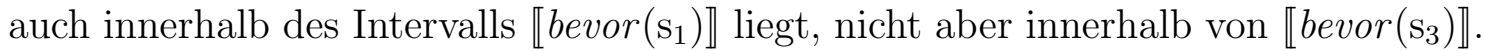
Aus $\phi\left[\right.$ bevor $\left.\left(\mathrm{s}_{1}\right)\right]$ folgt also nicht $\phi\left[\right.$ bevor $\left.\left(\mathrm{s}_{3}\right)\right]$. Umgekehrt kann das Telefonat auch mehrere Tage vor Marias Abitur liegen, es gilt also $\phi\left[\right.$ bevor $\left.\left(\mathrm{s}_{3}\right)\right]$. Daraus folgt aber, dass das Telefonat auch vor der Tibetreise liegt, aus $\phi\left[\right.$ bevor $\left.\left(\mathrm{s}_{3}\right)\right]$ folgt also auch $\phi\left[\right.$ bevor $\left.\left(\mathrm{s}_{1}\right)\right]$. Etabliert ist in diesem Fall nicht genau diese Menge an skalaren Ausdrücken, sondern vielmehr das Wissen um die Inklusion von kleineren Zeitintervallen in größeren. Zudem müssen die drei Situationsbeschreibungen $\mathrm{s}_{1}, \mathrm{~s}_{2}$ und $\mathrm{s}_{3}$ Teil des Common Ground sein. Es existiert mit $\phi\left[\right.$ bevor $\left.\left(\mathrm{s}_{3}\right)\right]$ also eine informativere Alternative zu der initialen Äußerung (354) (= $\phi\left[\right.$ bevor $\left.\left.\left(\mathrm{s}_{1}\right)\right]\right)$.

Angenommen, Marias Telefonat mit Peter hätte nun tatsächlich fünf Jahre vor ihrer Tibetreise stattgefunden (= Pfeil 3 in Abbildung 9.11). Dann bildet Satz (354) zwar eine semantisch korrekte Wiedergabe des Sachverhalts. Allerdings würde S mit dieser Äußerung gegen die 1. Submaxime der Quantität verstoßen, da S einen auf der HornSkala schwächeren Ausdruck gewählt hätte, obwohl in Form von $\left[\phi\left[\right.\right.$ bevor $\left.\left.\left(\mathrm{s}_{3}\right)\right]\right]$ eine informativere Alternative existiert. Nimmt H nun an, dass S die Griceschen Maximen befolgt, kann aus der Wahl des schwächeren Ausdrucks bevor $\left(\mathrm{s}_{1}\right)$ geschlossen werden, dass der stärkere Ausdruck bevor $\left(\mathrm{s}_{3}\right)$ nicht zutreffend ist bzw. S über das Zutreffen von bevor $\left(\mathrm{s}_{3}\right)$ nicht Bescheid weiß. Daraus lässt sich pragmatisch eine Einordnung 
des Hauptsatzereignisses an der in Abbildung 9.10 durch Pfeil 1 bezeichneten Intervallposition ableiten. Eine Verortung des Hauptsatzes an den Positionen 2 oder 3 wäre semantisch durchaus möglich. Sie wird aber dadurch ausgeschlossen, dass S, sollte eine solche Verortung intendiert gewesen sein, eine informativere Alternative gewählt hätte. Wäre der Hauptsatz beispielsweise wie Pfeil 2 einzuordnen, dann würde der Adverbialsatz bevor Maria umgezogen ist den informativsten Ausdruck bilden. Würde $\mathrm{S}$ dagegen wissen, dass das Telefonat vor fünf Jahren stattgefunden hat (= Pfeil 3), dann wäre die Variante bevor Maria ihr Abitur gemacht hat am informativsten. Da diese informativeren Alternativen von S nicht gewählt wurden, kann geschlossen werden, dass eine solche Wahl einen Verstoß gegen die Qualitätsmaxime bedeutet hätte. Der entsprechende Schlussprozess von H ist im Folgenden dargestellt (zu den Schritten (iii) und (iv) vgl. Kruijff-Korbayová 2010, 43).

\section{Rekonstruktion der skalaren Implikatur:}

(i) S hat $\phi\left[\right.$ bevor $\left.\left(\mathrm{s}_{1}\right)\right]$ geäußert.

(ii) Der Ausdruck bevor $\left(\mathrm{s}_{1}\right)$ ist Element einer Horn-Skala < bevor $\left(\mathrm{s}_{3}\right)$, be$\operatorname{vor}\left(\mathrm{s}_{2}\right)$, bevor $\left(\mathrm{s}_{1}\right)>$.

(iii) $\mathrm{Zu} \phi\left[\right.$ bevor $\left.\left(\mathrm{s}_{1}\right)\right]$ existieren Alternativen, nämlich alle Äußerungen des Typs $\phi\left[\right.$ bevor $\left.\left(\mathrm{s}_{1+n}\right)\right]$, die informativer wären als $\phi\left[\right.$ bevor $\left.\left(\mathrm{s}_{1}\right)\right]$.

(iv) Die zusätzliche Information in $\phi\left[\right.$ bevor $\left.\left(\mathrm{s}_{1+n}\right)\right]$ ist relevant für $\mathrm{H}$.

(v) $\phi\left[\right.$ bevor $\left.\left(\mathrm{s}_{1+n}\right)\right]$ ist nicht länger oder umständlicher als $\phi\left[\right.$ bevor $\left.\left(\mathrm{s}_{1}\right)\right]$. Es würde also bei Äußerung von $\phi\left[\right.$ bevor $\left.\left(\mathrm{s}_{1+n}\right)\right]$ kein Verstoß gegen die Modalitätsmaxime vorliegen.

(vi) Bei Befolgung der Quantitätsmaxime hätte $\mathrm{S} \phi\left[\right.$ bevor $\left.\left(\mathrm{s}_{1+n}\right)\right]$ äußern müssen, wenn $\phi\left[\right.$ bevor $\left.\left(\mathrm{s}_{1+n}\right)\right]$ mit der Qualitätsmaxime vereinbar ist.

(vii) Da $\mathrm{S}$ nicht $\phi\left[\right.$ bevor $\left.\left(\mathrm{s}_{1+n}\right)\right]$ geäußert hat, ist $\phi\left[\right.$ bevor $\left.\left(\mathrm{s}_{1+n}\right)\right]$ offenbar nicht mit der Qualitätsmaxime verträglich. Entweder weiß S nicht, ob $\phi\left[\right.$ bevor $\left.\left(\mathrm{s}_{1+n}\right)\right]$, oder:

(viii)S weiß, dass $\neg \phi\left[\right.$ bevor $\left.\left(\mathrm{s}_{1+n}\right)\right]$, und, insbesondere, S weiß, dass $\neg \phi[$ bevor $\left.\left(\mathrm{s}_{3}\right)\right]$.

Auch andere typische Merkmale von konversationellen Implikaturen treffen auf das Satzpaar in (355) zu. So ist die Implikatur B streichbar und kontextabhängig, wie das folgende Beispiel belegt. Nehmen wir an, die Diskursteilnehmer wissen, dass Peter und Maria telefoniert haben. Im Raum steht jedoch die Frage, wann dieses Telefonat stattgefunden hat. Peter und Maria hatten einen schlimmen Streit und gehen sich deswegen aus dem Weg, deswegen sind die Diskursteilnehmer daran interessiert zu erfahren, ob das Gespräch vor oder nach dem Streit war. S sagt:

Hm, Peter weiß nicht, wie es Maria in Tibet ergangen ist, also hat sie mit ihm telefoniert, bevor sie nach Tibet reiste, ja, sogar noch, bevor sie ihr Abitur gemacht hat, davon weiß er nämlich auch noch nichts.

In diesem Kontext implikatiert Satz (362) nicht, dass das Gespräch wenige Stunden oder Tage vor der Tibetreise stattfand. Außerdem kann die Implikatur bekräftigt 
werden, indem sie explizit formuliert wird. Dies trifft auf das folgende Beispiel zu.

Maria telefonierte mit Peter, bevor sie nach Tibet reiste, und zwar kurz vorher.

Bezogen auf Beispiel (354) kommt die zeitliche Nähe zwischen Haupt- und Nebensatz also rein pragmatisch zustande. ${ }^{68}$ Die temporale Proximität zwischen den beiden Teilsätzen kann zum Teil aber auch von der lexikalischen Semantik einzelner Subjunktionen gefordert sein (vgl. dazu u.a. Buscha 1989, Eisenberg et al. 2009, Eisenberg 1989, Pasch et al. 2003, Schilder 2004, 164 und Zifonun et al. 1997 für genaue Detailstudien einzelner Subjunktionen). So lässt sich beispielsweise feststellen, dass sowie im Unterschied zu nachdem eine zeitliche Nähe der beiden Ereignisse zueinander fordert. Aus diesem Grund ist Beispiel (364-b), in dem die PP die zeitliche Distanz der beiden Ereignisse zueinander verdeutlicht, anders als (364-a) nicht wohlgeformt.

a. Nachdem Peter an Weihnachten gefeuert wurde, wurde im nächsten Winter auch noch Karl rausgeschmissen.

b. \#Sowie Peter an Weihnachten gefeuert wurde, wurde im nächsten Winter auch noch Karl rausgeschmissen.

Um die im vorherigen Abschnitt dargestellte Semantik von temporalen Subjunktionen um diesen Aspekt zu erweitern, bieten sich zwei Möglichkeiten. Zum einen könnte man annehmen, dass sowie und nachdem unterschiedliche Anforderungen an die Positionierung des Hauptsatzes innerhalb des Intervalls Nachzeit stellen. So würde sowie beispielsweise fordern, dass der Hauptsatz zu Beginn der Nachzeit verortet werden muss. Gegen eine solche lexikalische Festlegung spricht jedoch, dass die temporale Relation zwischen Haupt- und Nebensatz im Rahmen der hier angenommenen Analyse nicht Teil der lexikalischen Bedeutung der jeweiligen Subjunktion ist. Da ein Temporalsatz ja nur indirekt eine zeitliche Relation zu seinem Matrixsatz herstellt, indem er einen Quantor restringiert, ist eine Spezifizierung dieser Relation im Lexikoneintrag der einzelnen Subjunktionen nicht möglich. Stattdessen soll hier ein anderer Lösungsweg eingeschlagen werden: Nachdem und sowie unterscheiden sich in der Art des durch sie gebildeten Intervalls. Während die Subjunktion nachdem angewandt auf die durch sie eingeleitete IP das Intervall Nachzeit ergibt, bildet sowie nur eine verkürzte Form dieses Intervalls. In Abbildung 9.12 wird gezeigt, dass das durch sowie gebildete Intervall in seinem rechten Rand stärker begrenzt ist als das entsprechende Denotat eines nachdem-Satzes.

\footnotetext{
${ }^{68}$ Die in diesem Abschnitt vorgestellte pragmatische Erklärung lässt sich auch ohne Weiteres auf eine zweistellige Semantik für temporale Subjunktionen übertragen. Nimmt man an, dass eine Subjunktion wie bevor eine Relation des Typs , <' einführt, stellt sich ebenfalls die Frage, wodurch in bestimmten Kontexten die zeitliche Nähe der beiden Ereignisse zustande kommt. Auch hier kann man argumentieren, dass eine skalare Implikatur durch die Wahl der Nebensatzproposition ausgelöst wird, wenn diese der Verortung des Hauptsatzes dienen soll.
} 


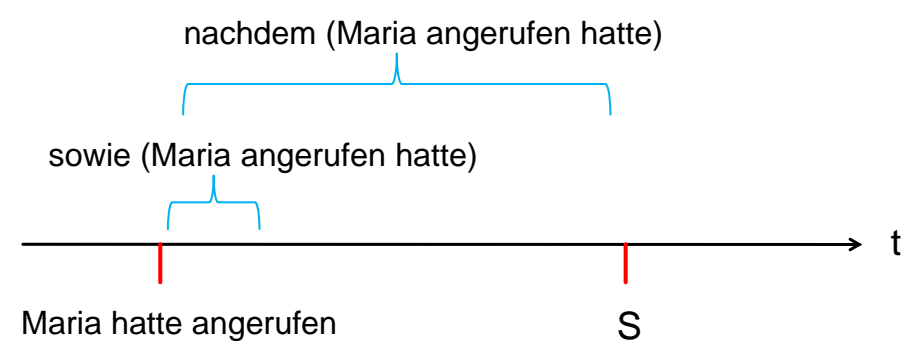

Abbildung 9.12.: Semantischer Unterschied zwischen nachdem und sowie

Diese Analyse deckt sich mit der Beschreibung des semantischen Beitrags von temporalen Präpositionen in Zifonun et al. (1997, 2108), die ebenfalls annehmen, dass das durch eine temporale Präposition gebildete Intervall unterschiedlich lang sein kann. Während die Präposition an beispielsweise Wochentage oder Feste als Komplement nimmt, bildet in längere Intervalle wie Monate oder Jahre. Der außensemantische Beitrag der beiden Präpositionen unterscheidet sich dagegen nicht, da das im Hauptsatz ausgedrückte Ereignis in beiden Fällen im jeweils denotierten Intervall enthalten sein muss.

Die Beobachtung, dass die durch einen Temporalsatz denotierten Intervalle in Abhängigkeit der lexikalischen Semantik der jeweiligen Subjunktion unterschiedlich lang sein können, zeigt, dass es sich bei den in (335) unterschiedenen drei Untergruppen an temporalen Subjunktionen nur um eine erste grobe Unterteilung handelt. Eine weitere, feinkörnigere Differenzierung der einzelnen Subjunktionen je nach Art des durch sie denotierten Intervalls ist erforderlich. Zudem spielt auch die Semantik des jeweils verwendeten Prädikats eine Rolle, wie die folgenden beiden Beispiele belegen.

(365) a. Nachdem Maria zu Mittag gegessen hatte, trank sie einen Espresso.

b. Nachdem Maria ihr Diplom gemacht hatte, ging sie nach London.

Die zeitliche Nähe bzw. Distanz zwischen Haupt- und temporalem Nebensatz wird durch zahlreiche Faktoren beeinflusst. In diesem Abschnitt wurde an einem Beispiel gezeigt, dass die zeitliche Proximität zwischen den beiden Sätzen in einigen Beispielen rein pragmatisch als skalare Implikatur abgeleitet werden kann. In anderen Fällen wird die zeitliche Nähe dagegen durch die lexikalische Semantik der Subjunktion gefordert. Beispielsweise bildet die Subjunktion sowie ein verkürztes Intervall. Schließlich kann auch die Wahl des Prädikats eine Rolle spielen. Bezüglich der Interaktion der unterschiedlichen Faktoren sind weitere Forschungen notwendig. Zudem muss noch eine feinkörnigere Differenzierung der temporalen Subjunktionen je nach Art des durch sie gebildeten Intervalls geleistet werden. 


\subsubsection{Zur Präsupposition von Temporalsätzen}

In den letzten Abschnitten wurde aufbauend auf den Ergebnissen von Johnston (1994) dafür argumentiert, dass es sich bei temporalen Subjunktionen um einstellige Ausdrücke handelt, die ihr IP-Komplement auf ein Zeitintervall abbilden. Dieses Intervall restringiert immer die Domäne eines overten oder koverten Quantors, in dessen Nukleus der Matrixsatz steht. Neben der Tatsache, dass diese Sichtweise eher den empirischen Daten aus Abschnitt 8 gerecht wird als eine relationale Analyse, liegt ein weiterer Vorteil des in den letzten Abschnitten entwickelten Ansatzes darin, dass aus ihm weitere spezifische Eigenschaften von Temporalsätzen abgeleitet werden können. So haben wir gesehen, dass Temporalsätze immer präsupponiert sind und keine Wurzelphänomene zulassen. Beide Punkte werden im Folgenden aus der hier vorgestellten nicht-relationalen Analyse von temporalen Subjunktionen abgeleitet. In diesem Abschnitt werden dabei zwei Ziele verfolgt: Zum einen wird argumentiert, dass sich der präsupponierte Status von Temporalsätzen direkt aus ihrer nicht-relationalen Semantik ergibt. Des Weiteren wird im Rahmen von SDRT gezeigt, dass die in einem Temporalsatz ausgedrückte Proposition in einem Diskurskontext immer durch ein direktes oder indirektes Antezedens lizenziert sein muss.

Beginnen wir mit der Frage, warum Temporalsätze präsupponiert sind. In Abschnitt 4.2 wurde u.a. Geurts (1999), Hara (2008), Heinämäki (1972), Johnston (1994), Kadmon (2001), Kamp/Reyle (1993), Karttunen (1973), Lakoff (1970), Lascarides/Oberlander (1993), Sawada/Larson (2004), Schilder/Tenbrink (2001), Tenbrink (2007) und Van der Sandt $(1992,342)$ folgend dafür argumentiert, dass die durch eine temporale Subjunktion eingeleitete Proposition präsupponiert ist. Präsupponierte Elemente wurden dabei also solche Elemente definiert, welche im Diskurskontext immer durch ein Antezedens lizenziert sein müssen. Dabei kann es sich entweder um die Bindung durch einen koreferenten Diskursreferenten oder aber um die Anknüpfung an ein indirektes Antezedens über eine Bridging-Relation handeln.

In dem folgenden Beispiel beschränkt der Temporalsatz die Domäne des Allquantors auf die Intervalle, für die gilt, dass Jenny in ihnen Zug fährt. Über diese Intervalle wird dann ausgesagt, dass Jenny in ihnen mit ihrem iPhone spielt, der Hauptsatz steht also im Nukleus des Allquantors.

(366) Jenny spielt immer mit ihrem iPhone, während sie Zug fährt.

Ist kein overter Quantor vorhanden, restringiert ein Temporalsatz einen koverten Quantor (vgl. dazu u.a. Johnston 1994 und Kratzer 1991). Temporal- und Matrixsatz bilden demnach die zwei Argumente eines overten oder koverten Quantors, wie in der folgenden Struktur dargestellt:

$$
\exists / \forall\{\text { Temporalsatz }\} \text { [Hauptsatz] }
$$

Interessanterweise ist es nun eine in der linguistischen Forschung weit verbreitete Annahme, dass Quantorrestriktionen immer einer Existenzpräsupposition unterliegen 
(vgl. beispielsweise Diesing 1992, Geurts/van der Sandt 1999, Larson 2004, Löbner 1987 und Roberts 1995). Betrachten wir dazu folgendes Beispiel aus Geurts/van der Sandt (1999). Die quantifizierte NP most passengers löst die Präsupposition aus, dass es eine kontextuell relevante Menge an Passagieren gibt. Dasselbe gilt für die NP in Beispiel (369).

(368) Most passengers survived the crash.

$\gg$ There were passengers.

(369) Alle Kinder schlafen.

$\gg$ Es gibt eine (kontextuell relevante) Menge an Kindern.

Geurts/van der Sandt (1999) argumentieren nun dafür, dass die durch Quantoren ausgelöste Präsupposition wie ein anaphorischer Ausdruck mit einem bereits gegebenen Referenten im Diskurskontext gleichgesetzt werden muss (vgl. die Bindungstheorie von Präsuppositionen in Abschnitt 4.1.3.2). Ein Satz wie (368) kann beispielsweise in einem Kontext wie dem folgenden geäußert werden, in dem die durch die quantifizierte NP ausgelöste Präsupposition durch ein Antezedens gebunden wird.

(370) The airplane carried some passengers. Most passengers survived the crash.

Auch von Fintel (2004a) nimmt an, dass Quantorrestriktionen in Form eines anaphorischen Indizes auftreten, der im Kontext gesättigt werden muss (vgl. dazu Fußnote (60) in Abschnitt 8.2). Löbner (1987) zeigt zudem, dass quantifizierte NPn in eine partitive Struktur mit einer Präposition und einem definiten Artikel umgeformt werden können wie in (371), wodurch ihre anaphorische Natur explizit gemacht wird.

The airplane carried some passengers. Most of the passengers survived the crash.

Die Präsupposition von Quantorrestriktionen kann auch im Kontext akkommodiert werden, wenn sie nicht durch ein Antezedens gebunden wird. Beispielsweise wird in der folgenden Äußerung die Präsupposition Es gab Passagiere über eine BridgingRelation zum Begriff Flugzeug interpretiert.

(372) Gestern ist in Sulawesi ein Flugzeug abgestürzt. Die meisten Passagiere haben zum Glück überlebt.

Genau wie ein Allquantor löst auch ein Existenzquantor eine Präsupposition aus. Ein Satz wie der folgende präsupponiert beispielsweise, dass es einen Sachverhalt x gibt, für den gilt, dass er komisch ist.

Etwas ist hier komisch.

Quantifizierte Strukturen der Form $\exists \mathrm{xP}(\mathrm{x})$ bzw. $\forall \mathrm{xP}(\mathrm{x})$ lösen demnach immer eine Existenzpräsupposition bezogen auf $\mathrm{x}$ aus. Welche Konsequenzen hat diese Annahme nun für die Analyse von Temporalsätzen? Larson (2004) und Sawada/Larson (2004) 
weisen erstmals auf den Zusammenhang zwischen der Funktion von Temporalsätzen als Quantorrestriktionen und ihrem präsupponierten Status hin. Sie argumentieren, dass Temporalsätze wie die in (374) genau wie die NPn in den Beispielen oben einer Präsupposition unterliegen, wie im Folgenden dargestellt.

a. Jenny spielt immer mit ihrem iPhone, während sie Zug fährt.

$\forall\{$ Temporalsatz [Hauptsatz]

$\gg$ Es gibt Situationen, für die gilt, dass Jenny in ihnen Zug fährt.

b. Jenny spielte mit ihrem iPhone, während sie Zug fuhr.

$\exists\{$ Temporalsatz $\}$ [Hauptsatz]

$\gg$ Es gibt mindestens eine Situation, für die gilt, dass Jenny in ihr Zug fährt.

Mit ihrer Funktion als Quantorrestriktion kann folglich die präsupponierte Natur von Temporalsätzen begründet werden. Attraktiv an diesem Ansatz ist insbesondere, dass die Entstehung der Präsupposition auf der Grundlage bereits unabhängig von der Beschäftigung mit Temporalsätzen erworbener Kenntnisse über quantifizierte Strukturen fußt und somit ohne spezifische Extraannahmen auskommt. Zudem ergibt sich aus dieser Sichtweise eine weitere zutreffende Vorhersage. In Abschnitt 8.2 wurde gezeigt, dass weil-Sätze nicht als Restriktionen in quantifizierten Strukturen in Frage kommen (vgl. Beispiel (327) in Abschnitt 8.2). Aus diesem Grund unterliegen sie auch keiner für Quantorrestriktionen typischen Existenzpräsupposition, weswegen sie grundsätzlich eingebettete Wurzelphänomene wie V2-Stellung im Deutschen aufweisen können.

Zusätzlich zu der hier skizzierten Begründung lässt sich die Präsupposition von Temporalsätzen auch pragmatisch ableiten. So argumentieren u.a. Geurts (1999), Kadmon (2001, 209ff) und Tenbrink (2004), dass Temporalsätze nur dann der zeitlichen Einordung des Hauptsatzes dienen können, wenn das in ihnen ausgedrückte Ereignis bereits bekannt und auf dem Zeitstrahl verortet ist. Derselbe Gedanke lässt sich auch im Rahmen der hier vorgeschlagenen nicht-relationalen Analyse von Temporalsätzen fassen: Die in einem Temporalsatz ausgedrückte Ereignisbeschreibung s bildet den rechten und/oder linken Rand des gebildeten Zeitintervalls i. Beispielsweise markiert der in dem Temporalsatz ausgedrückte Sachverhalt s in der folgenden Äußerung den rechten Rand des denotierten Intervalls $i=$ Vorzeit (vgl. Abbildung 9.4 in Abschnitt 9.1.1).

Bevor Martina nach Chile fliegt, muss sie einen Pass beantragen.

Das entsprechende Intervall i kann nun aber nur gebildet werden, wenn s = Martina fliegt nach Chile wahr und auf dem Zeitstrahl verankert ist, denn nur so kann s das Intervall in seinem rechten Rand begrenzen. Wäre s nicht wahr und zum Äußerungszeitpunkt nicht bereits gegeben, wäre es konversationell nicht sinnvoll, s als Grundlage zur Bildung von i zu verwenden. Zusätzlich zu der oben beschriebenen Erklärung der mit einem Temporalsatz verbundenen Präsupposition kann diese folglich auch auf der Grundlage von pragmatischen Prinzipien abgeleitet werden. 
Dass die in einem Temporalsatz ausgedrückte Proposition präsupponiert ist, hat Konsequenzen für die Verwendung eines Temporalsatzes in einem Diskurs. Genauer gesagt ist die Äußerung eines Temporalsatzes nur dann wohlgeformt, wenn sein Inhalt entweder durch ein koreferentes Antezedens gebunden wird oder aber über eine Bridging-Relation durch ein indirektes Antezedens lizenziert wird. Da eine temporale Subjunktion einen präsupponierten Satz einleitet, generiert das präsupponierte IP-Komplement eine vom assertierten Satzinhalt separierte Struktur (vgl. dazu die Diskussion der Behandlung von Präsuppositionen in SDRT in Abschnitt 4.1.3.3). Ein komplexer Satz wie (376) besteht demnach aus zwei separaten SDRS-Boxen, wie in (377) dargestellt. Die linke Struktur enthält den assertierten Hauptsatz, wohingegen die präsupponierte Adverbialsatzproposition in der rechten Box verarbeitet wird. Innerhalb der präsupponierten Struktur wird eine unspezifizierte Diskursrelation R generiert, deren erstes oder zweites Argument die präsupponierte Proposition $\pi_{p}$ bildet (vgl. dazu auch Lascarides/Oberlander 1993, Schilder 1999 und Schilder/Tenbrink 2001, die ebenfalls davon ausgehen, dass Temporalsätze aufgrund ihres präsupponierten Status eine unspezifizierte Relation $\mathrm{R}$ generieren). ${ }^{69}$

Nachdem er seine Mutter besucht hatte, ging Peter ins Kino.
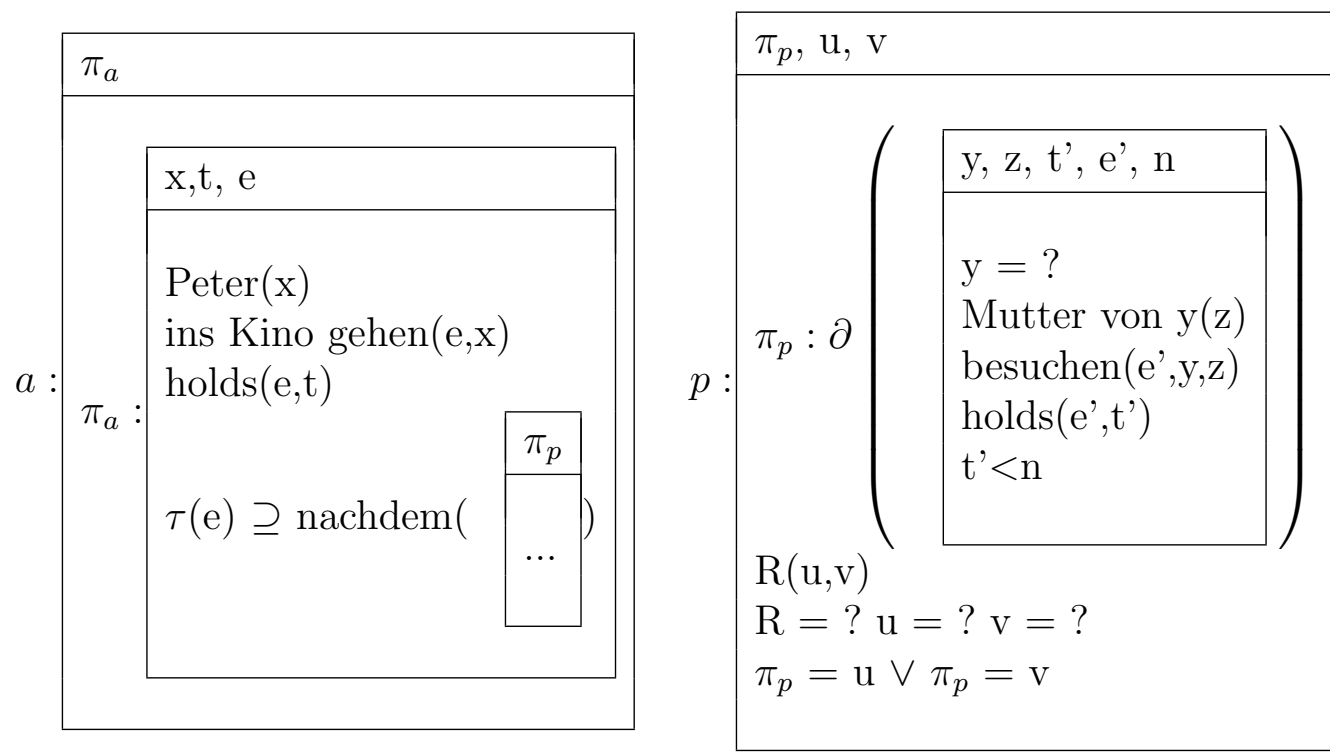

In dieser Darstellung ist die temporale Subjunktion nicht Teil des präsupponierten, sondern des assertierten Inhalts. Dies ist damit zu begründen, dass nicht der gesamte Nebensatz, sondern nur die uneingeleitete Nebensatzproposition präsupponiert ist. Der einstellige Ausdruck nachdem nimmt als Teil der Assertion nach Verarbeitung der präsupponierten SDRT-Struktur die Proposition als Argument und bildet diese auf ein Intervall ab. Mithilfe der Laufzeit-Funktion $\tau$ wird die Zeitspanne, die das Hauptsatzereignis, also Peters Kinobesuch, in Anspruch nimmt, ermittelt. Das Ergebnis muss innerhalb des Intervalls, welches aus der funktionalen Applikation von

\footnotetext{
${ }^{69}$ Der besseren Lesbarkeit halber wird auf die Darstellung der Präsuppositionen, welche durch die definiten NPn ausgelöst werden, verzichtet.
} 
nachdem und der präsupponierten Proposition resultiert, enthalten sein bzw. diesem entsprechen.

Wie im vorherigen Abschnitt gezeigt, muss die präsupponierte Struktur nun in einem zweiten Schritt in die assertierte Struktur aufgenommen werden. Dabei müssen die bis dahin unspezifizierte Diskursrelation $\mathrm{R}$ aufgelöst und ihre beiden Argumente festgelegt werden. Wie definite NPn kann auch die Proposition eines Temporalsatzes durch ein inhaltlich identisches Antezedens gebunden werden. Der aus Beispiel (376) bereits bekannte Satz ist in (378) in einen längeren Diskurs eingebettet. Entscheidend ist, dass die im Temporalsatz enthaltene Proposition $\pi_{p}$ im Kontext mit $\pi_{2}$ ein Antezedens mit demselben Inhalt besitzt.

$\pi_{1}$ : Gestern hatte Peter viel vor.

$\pi_{2}$ : Zum ersten Mal seit langer Zeit hat er seine Mutter besucht.

$\pi_{3}$ : Sie machten einen kleinen Spaziergang.

$\pi_{4}$ : Mittags ging er mit ihr in ein italienisches Restaurant.

$\pi_{5}$ : Nachdem $\left(\pi_{p}\right.$ : er seine Mutter besucht hatte, $)$ ging er ins Kino.

Die durch nachdem eingeleitete Proposition generiert die in (377) dargestellte separate Struktur, welche eine unspezifizierte Relation $R$ sowie deren unspezifizierte Argumente $\mathrm{u}$ und v enthält. Beide Formen der Unbestimmtheit können erst im jeweiligen Kontext aufgelöst werden. Bezogen auf den Diskurskontext (378) wird die im Temporalsatz enthaltene Proposition durch ein inhaltlich identisches Antezedens gebunden. So, wie eine definite NP durch ein indefinites Antzedens eingeführt werden kann, wird der Temporalsatz $\pi_{p}$ in Diskurs (378) mit dem Antezedens $\pi_{2}$ gleichgesetzt. Folglich wird die unspezifizierte Relation $\mathrm{R}$ als Coreference übersetzt, zudem werden die Argumentstelle $\mathrm{u}$ durch die präsupponierte Proposition des Temporalsatzes $\pi_{p}$, und die Argumentstelle v mit $\pi_{2}$ gesättigt. Die Struktur des gesamten Diskurses in (378) ist in (379) nach Verarbeitung der Präsupposition vereinfacht dargestellt.

In der Darstellung ist zu erkennen, dass die initiale Aussage $\pi_{1}$ durch alle folgenden Sätze, welche als $\pi_{6}$ zusammengefasst sind, weiter elaboriert wird. Außerdem bilden $\pi_{2}$ und $\pi_{5}$ eine Erzählkette von aufeinander folgenden Ereignissen. Dasselbe gilt für $\pi_{3}$ und $\pi_{4}$, welche zudem die Proposition $\pi_{2}$ weiter ausführen. Wie ein Pronomen oder eine definite NP greift der präsupponierte Temporalsatz in dem Diskurs oben ein Antezedens auf, mit dem er koreferent ist. Dabei referieren die assertierte Äußerung $\pi_{2}$ und die Präsupposition $\pi_{p}$ nicht auf eine Entität, sondern auf ein Ereignis. Durch die Assertion $\pi_{2}$ wird dieses Ereignis erstmals in den Diskurs eingeführt und es wird ein Diskursreferent $\pi_{2}$ etabliert, den der Temporalsatz wieder aufgreift. 


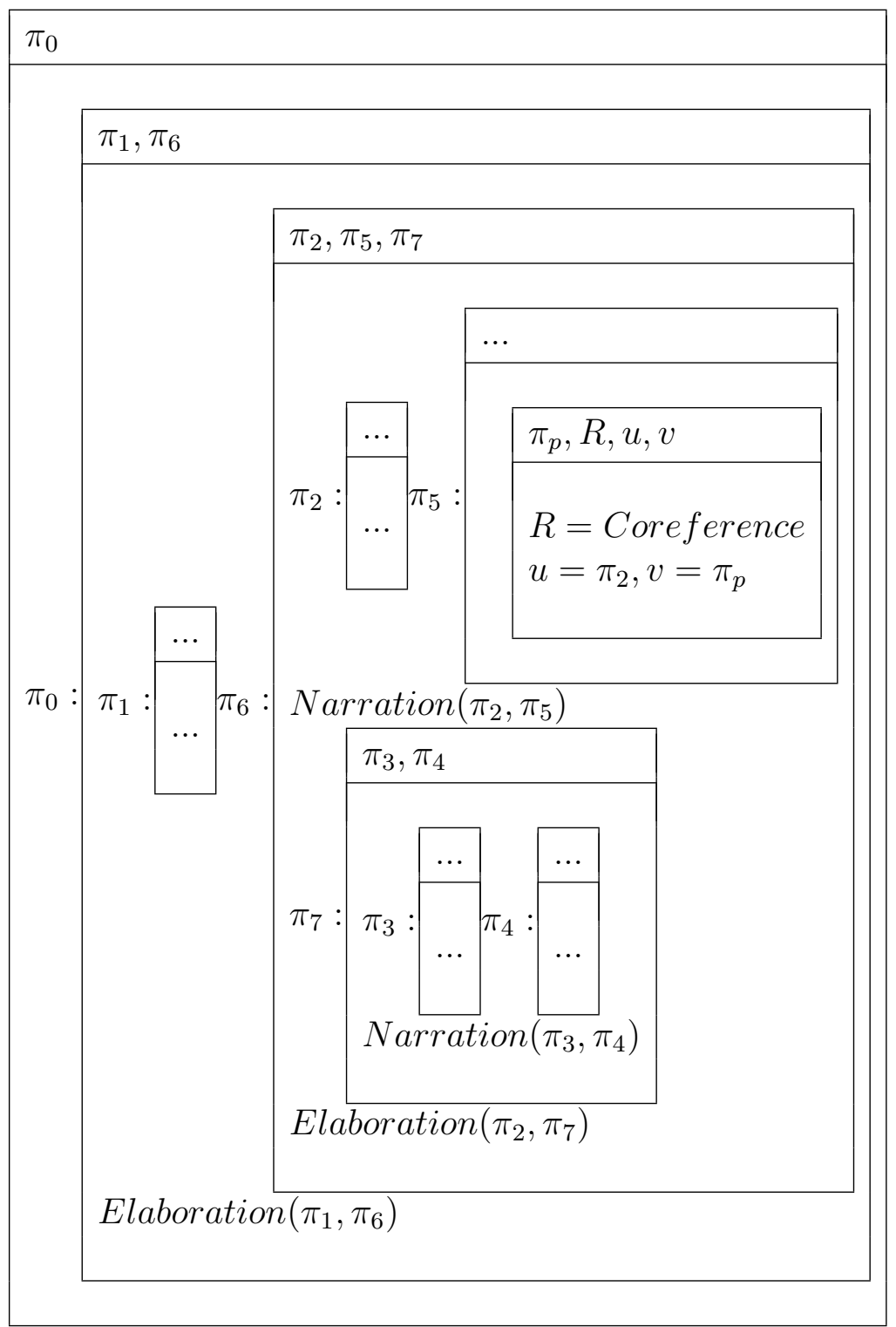

Fälle, in denen ein Temporalsatz einen zuvor eingeführten Diskursreferenten aufgreift, lassen sich auch in der tatsächlichen Sprachverwendung finden, wie der folgende Originalbeleg zeigt (aus: Der SPIEGEL 47/2010, 110). Auch hier führt ein uneingebetteter Satz den relevanten Diskursreferenten ein, durch welchen der nachfolgende Temporalsatz gebunden wird.

(380) (...) Wenige Monate nach seinem erzwungenen Abschied als Chef des deutschen Software-Unternehmens SAP wurde Léo Apotheker zum Boss des Silicon-Valley-Riesen Hewlett-Packard (HP) ernannt. [...] Nachdem der US-Konzern Apotheker zum Chef ernannt hatte, beschloss Oracle, den ehemaligen SAP-Mann in den Zeugenstand zu rufen, um ihn als Dieb zu brandmarken. 
Greift ein Temporalsatz ein direktes Antezedens mit der Relation Coreference auf, wirkt dies häufig redundant. Beispielsweise würde man in dem folgenden Diskurs wahrscheinlich eher dem Gebrauch eines temporalen Adverbs wie danach als der Wiederholung der Proposition in Form eines Temporalsatzes den Vorzug geben.

(381) Peter war gestern im Kino. Bevor er im Kino war/Davor hat er seine Mutter besucht.

Die Präferenz des Pronominalausdrucks gegenüber dem Temporalsatz in Beispiel (381) kann mithilfe des Prinzips Maximize Presupposition nach Heim (1991) erklärt werden (vgl. dazu die Diskussion in Abschnitt 4.1.3.2). Da das Temporaladverb anders als der Temporalsatz über keinen eigenen semantischen Inhalt verfügt und somit in weniger Kontexten verwendet werden kann, bildet es einen stärkeren Präsuppositionsausdruck (vgl. dazu (170) in Abschnitt 4.1.3.2). Nach dem Prinzip Maximize Presupposition ist die Verwendung von davor in Beispiel (381) der Verwendung des Temporalsatzes demnach klar zu bevorzugen. Auch das Pronominaladverb davor enthält eine anaphorische Komponente, die durch ein Antezedens gebunden werden muss. Anders als nicht-pronominale Anaphern werden Pronominalausdrücke bis auf wenige Ausnahmen aber nur durch ein koreferentes Antezedens lizenziert. Temporalsätze, welche als präsupponierte Ausdrücke ebenfalls durch den vorherigen Diskurs lizenziert sein müssen, können dagegen auch über eine andere Relation als $\mathrm{R}=$ Coreference angebunden werden. Da sie anders als Pronominalausdrücke über einen reichen semantischen Inhalt verfügen, kann die in ihnen ausgedrückte Proposition auch mit einem indirekten Antezedens verknüpft werden. So drückt der Temporalsatz in dem folgenden Beispieldiskurs eine Proposition aus, für die im direkten Diskurskontext noch kein Diskursreferent etabliert wurde.

Die Gäste sollten um acht Uhr kommen und Peter wollte ein richtiges Festmahl kochen. Nachdem er den Reis aufgesetzt hatte, gönnte er sich aber erst einmal eine Zigarette auf dem Balkon.

Wir haben bereits in Abschnitt 4.1.3.3 gesehen, dass im Rahmen von SDRT davon ausgegangen wird, dass Präsuppositionen, die eine neue Information einführen, über eine Diskursrelation mit dem Kontext verknüpft werden müssen (vgl. dazu Asher/Lascarides 1998b und Asher/Lascarides 2003). In Beispiel (382) steht das im Temporalsatz ausgedrückte Ereignis des Reiskochens in einer Inklusionsbeziehung zu dem im vorherigen Satz evozierten Ereignis der Festmahlzubereitung. Demnach wird der Temporalsatz in diesem Fall mit der Diskursrelation $\mathrm{R}=$ Sub-Part angebunden (vgl. dazu auch Schilder 1999, der ebenfalls argumentiert, dass englische Temporalsätze über eine Teil-Ganzes-Beziehung mit einem Antezendens verknüpft werden können). Auch in dem folgenden Originalbeleg (aus: Julia Franck 2007: Die Mittagsfrau, 16) wird durch den vorherigen Diskurs das Frame Trinken eröffnet. Den Wasserhahn zu öffnen kann nun in einer Teil-Ganzes-Beziehung zu diesem Ereignis stehen, es gilt demnach auch hier $\mathrm{R}=$ Sub-Part. 
(383) (...) Er hatte Durst. Lange Zeit fühlte er sich zu schwach, um aufzustehen und zum Spülbecken zu gehen. Als er den Wasserhahn aufdrehte, hörte er nur ein Röcheln, kein Wasser kam.

Auch in Beispiel (384) aus dem SPIEGEL (47/2010, 137f) wird der Temporalsatz nicht durch ein koreferentes Antezedens gebunden. Vielmehr führt er die im vorherigen Satz beschriebene Handlung weiter, indem er die Landung der Bomber, deren Inspektionsflüge zuvor geschildert wurden, nennt. Es gilt folglich $\mathrm{R}=$ Narration .

(...) Der heute 72-Jährige blieb über Monate im Pazifik, um die Tests zu fotografieren. Er erinnert sich, wie leichtfertig das Militär damals operierte: „Nach der Detonation flogen immer B-57-Bomber direkt durch den Atompilz, um Messungen durchzuführen. Nachdem die Maschinen wieder gelandet waren, wurden sie von Männern dekontaminiert, die als einzigen Schutz Gummistiefel an den Füßen trugen."

In Abschnitt 4.1.3.3 haben wir bereits gesehen, dass Asher/Lascarides (1998b) und Asher/Lascarides (2003) im Rahmen von SDRT anders als Van der Sandt (1992) keinen Unterschied zwischen der Bindung und der Akkommodation einer Präsupposition machen. In beiden Fällen muss der präsupponierte Inhalt über eine unspezifizierte Diskursrelation $\mathrm{R}$ an den Kontext angebunden werden. Dies können wir nun am Beispiel der Temporalsätze etwas genauer formulieren: Ist im Diskurskontext ein direktes Antezedens gegeben, wird das IP-Komplement einer temporalen Subjunktion so wie in Beispiel (378) mit diesem über die Diskursrelation $\mathrm{R}=$ Coreference gleichgesetzt. Führt der Temporalsatz dagegen die Beschreibung eines diskursneuen Ereignisses ein, muss der Satz über eine andere Relation wie beispielsweise $S u b$ Part oder Narration mit einem indirekten Antezedens verknüpft werden. Von der Forschung ist dabei noch zu zeigen, welche Werte $\mathrm{R}$ annehmen kann und ob diese mit einer unterschiedlich hohen Präferenz gewählt werden. Schilder/Tenbrink (2001) haben in einer ersten Korpusrecherche das Verhalten von englischen before- und after-Sätzen untersucht und kommen dabei zu dem Schluss, dass die Sätze präferiert über die Relation Elaboration angebunden werden, wobei sie die Relation Sub-Part als einen Sonderfall der übergeordneten Relation Elaboration betrachten. Weitere empirische Untersuchungen sind hier aber noch notwendig.

Der einzige Unterschied zwischen der Akkommodation und der Bindung einer Präsupposition besteht demnach in dem Wert, den $\mathrm{R}$ annimmt. Hierin unterscheidet sich die vorliegende Analyse wesentlich von der Untersuchung von Lascarides/Oberlander (1993), die davon ausgehen, dass die Diskursanbindung einer Präsupposition über eine Relation R nur dann zum Tragen kommt, wenn die Präsupposition nicht gebunden werden kann. Problematisch an dieser Sichtweise ist jedoch, dass unklar bleibt, ob präsupponierte Inhalte immer eine unspezifizierte Relation $\mathrm{R}$ generieren, die im Falle einer Bindung nicht gebraucht wird, oder ob $\mathrm{R}$ erst in einem zweiten Schritt generiert wird, wenn die Bindung der Präsupposition scheitert. Wird auch die Bindung von Präsuppositionen als Diskursanbindung über eine Relation $\mathrm{R}=$ Coreference modelliert, wie hier vorgeschlagen, stellt sich dieses Problem dagegen 
nicht.

Kann dagegen keine Relation zu einem koreferenten oder einem indirekten Antezedens hergestellt werden, scheitert die Diskursanbindung der Präsupposition und die Äußerung ist nicht wohlgeformt. Dies trifft auf das folgende Beispiel zu, wo der Inhalt des Temporalsatzes eine Information ausdrückt, die durch den Kontext nicht lizenziert ist (vgl. auch die Beispiele (189) und (190) in Abschnitt 4.2).

A: Was machst du in den Sommerferien?

B: ?Bevor ich in die Toskana fahre, muss ich noch ein paar Reparaturen am Haus erledigen.

Bereits in Abschnitt 4.2 wurde dafür argumentiert, dass Temporalsätze präsupponiert sind. Diese Annahme wurde in diesem Abschnitt nun mit dem spezifischen semantischen Beitrag von Temporalsätzen begründet. Temporale Subjunktionen sind einstellige Operatoren, die zusammen mit ihrem IP-Komplement die Beschreibung eines Zeitintervalls bilden. Die temporale Relation zwischen Hauptund Adverbialsatz kommt dann dadurch zustande, dass beide stets Argumente eines overten oder koverten Quantors bilden. Dabei restringiert der Temporalsatz die Domäne des Quantors auf das oder die durch ihn denotierten Intervalle. Aus ihrer Funktion als Quantorrestriktionen folgt dann auch der präsupponierte Status von Temporalsätzen, da Quantorrestriktionen grundsätzlich eine Existenzpräsupposition zugrunde liegt. Zudem ist es möglich, die durch einen Temporalsatz ausgelöste Präsupposition pragmatisch zu rekonstruieren. Da temporale Adverbialsätze präsupponiert sind, können sie auch nicht zum Ausdruck von [+at-issue]-Inhalten verwendet werden. Dies ergibt sich direkt aus der Argumentation in Kapitel 4, derzufolge Präsuppositionen eine Untermenge von [-at-issue]-Inhalten bilden. Da Temporalsätze als Präsuppositionen nicht zum Ausdruck von [+at-issue]-Inhalten geeignet sind, können sie im Deutschen auch keine V2-Stellung aufweisen, welche ja nur in den Nebensätzen möglich ist, die der Beantwortung der aktuellen Q dienen. Auch im Englischen blockieren Temporalsätze Wurzelphänomene, was in diesem Fall direkt auf das Merkmal [+präsupponiert] zurückzuführen ist (vgl. dazu Abschnitt 7.3.2). Nach dieser ausführlichen Untersuchung des semantischen Beitrags von temporalen Adverbialsätzen und deren Platzierung in einem Diskurs wird im Folgenden der Vollständigkeit halber eine semantische Beschreibung des kausalen Konnektors weil erfolgen.

\subsection{Weil-Sätze}

Die Untersuchung von Temporalsätzen in negierten und quantifizierten Strukturen in Abschnitt 8 hat ergeben, dass sich diese nicht wie relationale Ausdrücke verhalten, weshalb in den vorherigen Abschnitten aufbauend auf den Ergebnissen von Johnston (1994) eine alternative Analyse des semantischen Beitrags von temporalen Subjunktionen vorgeschlagen wurde. Anders als Temporal- haben weil-Sätze dagegen tatsächlich das Verhalten gezeigt, wie man es von relationalen Strukturen, die 
noch eine ungesättigte Argumentstelle besitzen, erwarten würde. Dies deckt sich mit der traditionellen Auffassung, derzufolge weil eine zweistellige kausale Relation einführt, weswegen die folgende Darstellung des semantischen Beitrags von weilSätzen deutlich kürzer ausfallen wird als die Untersuchung von Temporalsätzen in den vorherigen Abschnitten.

Nach u.a. Blühdorn (2006), Eisenberg (2006), Keller (1993), Lohnstein/Bredel (2004), Max (2004), Pasch et al. (2003), Pittner (1999), Scheffler (2005), Thim-Mabrey (1982) und Zifonun et al. (1997) führt die Subjunktion weil eine zweistellige Relation zwischen Haupt- und Nebensatz ein. Weil ist demnach vom Typ $<\mathrm{t},<\mathrm{t}, \mathrm{t}>>$, da es zur Sättigung zwei Propositionen benötigt und diese auf einen Wahrheitswert abbildet. Zur Darstellung der kausalen Relation wird im Folgenden der zweistellige Ausdruck CAUSE(p,q) verwendet. Nach Eisenberg (2006, 335) unterliegt CAUSE denselben Wahrheitsbedingungen wie der logische Konnektor $\wedge$, da ein komplexer Satz bestehend aus einem weil- und seinem Bezugssatz nur dann wahr ist, wenn es beide Teilsätze sind. Im Unterschied zu $\wedge$ ist in der lexikalischen Semantik von weil jedoch festgelegt, dass die beiden verknüpften Sätze in einer Kausalrelation zueinander stehen. Dabei erhält nach Blühdorn (2006) das interne Konnekt p die Rolle URSACHE, GRUND (EVIDENZ) oder MOTIV, wohingegen das externe Konnekt q die Relationsrolle WIRKUNG oder FOLGE(RUNG) zugewiesen bekommt. Eine Besonderheit des Konnektors weil besteht zudem darin, dass das externe Argument q unterschiedlich besetzt werden kann, was noch begünstigt wird, wenn der weil-Satz mit V2-Stellung gebildet wird. Je nachdem, ob der Kausalsatz den propositionalen Inhalt seines Bezugssatzes, die epistemische oder die Sprechaktebene modifiziert, erhalten wir unterschiedliche Lesarten, die von u.a. Antomo/Steinbach (2010), Blühdorn (2006), Breindl (2009), Gaumann (1983), Holler (2008), Keller (1993), Küper (1991), Pasch et al. (2003), Pasch (1997), Pittner (1999), Uhmann (1998), Thim-Mabrey (1982), Volodina (2011) und Wegener (1993) bereits ausführlich diskutiert wurden.

Zentral ist nun, dass mit weil eingeleitete Sätze anders als Temporalsätze nicht grundsätzlich präsupponiert sind, auch wenn sie in spezifischen Kontexten natürlich eine präsupponierte Information ausdrücken können. Nach u.a. Blühdorn (2006) und Pasch et al. (2003) unterscheidet sich weil in diesem Punkt von der kausalen Subjunktion $d a$, welche immer einen präsupponierten Nebensatz einleitet. In einem komplexen Satzgefüge bestehend aus einem weil- und seinem Bezugssatz können nicht nur beide Teilsätze eine dem Hörer unbekannte Information ausdrücken, es ist sogar möglich, dass der Matrixsatz präsupponiert ist und der Kausalsatz allein die assertierte Information enthält. Bereits Hooper/Thompson (1973) beobachten, dass mit because eingeleitete Sätze im Englischen assertiert sein können, während ihr Matrixsatz eine präsupponierte Information ausdrückt (vgl. dazu auch Larson 2004). Dies trifft auch auf deutsche weil-Sätze zu, was u.a. Blühdorn (2006) und Thim-Mabrey $(1982,207 f f)$ damit belegen, dass ein weil- anders als ein da-Satz ohne Bezugssatz zur Beantwortung einer warum-Frage verwendet werden kann, wie in dem folgenden Beispiel zu sehen ist: 
Warum haben Peter und Maria gestritten?

a. Weil er nicht gespült hat.

b. ?Da er nicht gespült hat.

In (386) ist der im Hauptsatz ausgedrückte Sachverhalt bereits bekannt, weswegen er in den beiden Antworten weggelassen wird. Dagegen drückt die Begründung eine neue Information aus, die assertiert wird. Die Verwendung eines $d a$-Satzes ist in diesem Kontext nicht wohlgeformt, was Blühdorn (2006) darauf zurückführt, dass $d a-$ Sätze immer präsupponiert sind und nicht zum Ausdruck einer diskursneuen Information geeignet sind. Aufbauend auf den Ergebnissen von Teil I können wir zudem festhalten, dass mit $d a$ eingeleitete Sätze offensichtlich nicht [+at-issue]-Inhalt enthalten können, was sich mit den Ergebnissen von Averintseva-Klisch/Volodina (2012) deckt. Weil-Sätze können dagegen unter Ellipse des Bezugssatzes verwendet werden, was belegt, dass sich weil-Sätze zum Ausdruck einer diskursneuen Information eignen. Gleichzeitig kann ein weil-Satz aber auch zum Ausdruck einer bekannten Information verwendet werden, wie das folgende Beispiel belegt:

A: Du gehst ins Restaurant, obwohl Peter heute kochen will?

B: Ich gehe ins Restaurant, WEIL Peter heute kochen will.

In Beispiel (387) beziehen sich Haupt- und Kausalsatz auf im Common Ground bereits gegebene Informationen. Neu ist lediglich die Betonung, dass die beiden Sachverhalte in einer kausalen Relation zueinander stehen. Dies zeigt, dass ein mit weil eingeleiteter Satz auch präsupponiert sein kann. Im Unterschied zu da kann weil aber auch einen assertierten Satz einleiten.

Anders als temporale Subjunktionen leiten kausale demnach nicht grundsätzlich einen präsupponierten Nebensatz ein. Vielmehr scheint bei den kausalen Konnektoren eine lexikalische Ausdifferenzierung vorzuliegen: $D a$-Sätze sind immer präsupponiert, denn-Sätze sind assertiert und weil kann als am wenigsten spezialisierter kausaler Konnektor entweder einen dem Hörer bekannten oder einen diskursneuen Sachverhalt einleiten (vgl. dazu u.a. Blühdorn 2006, Pasch et al. 2003 und Wegener 2000). Nun stellt sich die Frage, womit dieser Unterschied zwischen kausalen und temporalen Subjunktionen zusammenhängt. Genauer gesagt: Aus welchem Grund selegieren temporale Subjunktionen immer ein präsupponiertes Komplement, kausale dagegen nicht? Eine rein lexikalische Festlegung der Präsuppositionseigenschaften in den Lexikoneinträgen der jeweiligen Subjunktionen wird der Tatsache nicht gerecht, dass es sich dabei um ein sprachübergreifend stabiles Phänomen handelt, wie in Abschnitt 7.3.2 angedeutet wurde. Mit der Beantwortung dieser Frage hängt letztendlich auch die Frage zusammen, weshalb Temporalsätze nicht zum Ausdruck von [+at-issue]-Inhalt geeignet sind, weil-Sätze dagegen schon. Da Präsuppositionen als eine Untermenge von [-at-issue]-Inhalten analysiert wurden (vgl. dazu die Diskussion in Abschnitt 4), ergibt sich aus dem Merkmal [+präsupponiert] auch automatisch die Eigenschaft [-at-issue]. Umgekehrt gilt für Bedeutungsaspekte, die [-präsupponiert] sind, dass sie Teil der Hauptstruktur sein können, wenngleich das 
Merkmal [-präsupponiert] noch kein hinreichendes Kriterium für At-issueness ist. Dass die in einem Temporalsatz ausgedrückte Proposition immer präsupponiert ist, wurde in Abschnitt 9.1.3 damit begründet, dass Temporalsätze immer als Restriktion eines overten oder koverten Quantors fungieren. Der allgemeine Aufbau einer quantifizierten Struktur ist in (388-a) und das Verhalten eines komplexen Satzgefüges bestehend aus einem Temporal- und seinem Bezugssatz in (388-b) stark vereinfacht wiederholt.

$$
\begin{aligned}
& \text { a. } \quad \forall / \exists\{\text { Restriktion (+präsupponiert })\}[\text { Nukleus] } \\
& \text { b. } \quad \forall / \exists\{\text { Temporalsatz }\}[\text { Matrixsatz } \wedge \mathrm{f}(\mathrm{e}) \subseteq \mathrm{i}]
\end{aligned}
$$

Da Temporalsätze immer die Restriktion eines overten oder koverten Quantors bilden, unterliegen sie einer Existenzpräsupposition, womit in Abschnitt 9.1.3 ihr präsupponierter Status erklärt wurde. Betrachten wir nun weil-Sätze. Wir haben in Abschnitt 8.2 bereits gesehen, dass ein weil-Satz nicht dazu verwendet werden kann, die Domäne eines Quantors zu restringieren. Ein Beispiel wie das folgende kann aber auf zwei Weisen interpretiert werden, wobei die beiden möglichen Lesarten in (390) dargestellt sind.

(389) Peter hat immer geraucht, weil er nervös war.

a. Lesart 1:

In allen relevanten Situationen hat Peter geraucht und er hat es deswegen getan, weil er nervös war.

b. Lesart 2:

In allen Situationen, in denen Peter geraucht hat, hat er es deswegen getan, weil er nervös war.

Entscheidend ist nun, dass der Allquantor in keiner der beiden Lesarten durch den Kausalsatz restringiert wird. Vielmehr ist die Restriktion in Lesart 1 nicht overt realisiert und der Gesamtsatz bildet den Nukleus des Allquantors, wie in (391-a) dargestellt. Die der zweiten Lesart zugrundeliegende Struktur ist dagegen in (391-b) abgebildet. Hier bildet der Matrixsatz die Restriktion und beschränkt die Domäne des Allquantors auf solche Situationen, für die gilt, dass Peter in ihnen raucht.

a. Lesart 1:

$\forall\{\}$ [CAUSE(rauchen(Peter), nervös sein(Peter))]

b. Lesart 2:

$\forall\{$ rauchen $($ Peter $)\}$ [nervös sein (Peter) $\wedge$ CAUSE(rauchen(Peter), nervös sein(Peter))]

Wir können also festhalten, dass weil-Sätze im Gegensatz zu Temporalsätzen keiner grundsätzlichen Existenzpräsupposition unterliegen, da sie nicht als Quantorrestriktionen fungieren. Natürlich sind aber nicht alle Präsuppositionen darauf zurückzuführen, dass der entsprechende Ausdruck einen Quantor restringiert. Wie in Kapitel 4 gezeigt wurde, kann eine Präsupposition auch lexikalisch, dies trifft beispiels- 
weise auf den definiten Artikel zu, oder pragmatisch ausgelöst werden. Bezogen auf die Präsupposition von temporalen Adverbialsätzen wird eine solche pragmatische Herleitung beispielsweise von Geurts (1999), Kadmon (2001, 209ff) und Tenbrink (2004) vertreten, wie wir in Abschnitt 9.1.3 gesehen haben. Deren Annahme, dass ein Temporalsatz nur dann dazu dienen kann, den Haupsatz temporal einzuordnen, wenn er selbst bereits gegeben und auf dem Zeitstrahl positioniert ist, erscheint dabei sehr plausibel und kann die in Abschnitt 9.1.3 vorgeschlagene Ableitung der Präsupposition von Temporalsätzen aus ihrer Funktion als Quantorrestriktionen ergänzen. Im Gegensatz dazu lässt sich keine pragmatische Argumentation finden, aufgrund derer ein weil-Satz eine grundsätzliche Präsupposition auslösen sollte. Anders als ein Temporalsatz muss ein weil-Satz nicht zwangsläufig einen im Diskurs bereits gegebenen Sachverhalt ausdrücken, damit die Gesamtäußerung interpretierbar ist. So kann der Kausalsatz in dem folgenden Beispiel problemlos eine Information ausdrücken, die in keiner Weise in dem vorherigen Diskurs verankert ist, dennoch kann diese als Begründung für den im Hauptsatz ausgedrückten Sachverhalt angeführt werden.

Maria kommt heute nicht zur Vorlesung, weil sie krank ist.

Da es sich bei weil-Sätzen nicht um Quantorrestriktionen handelt und auch keine pragmatische Notwendigkeit dafür, dass der in einem weil-Satz ausgedrückte Sachverhalt zum Äußerungszeitpunkt bereits gegeben sein muss, besteht, unterliegen weil-Sätze anders als Temporalsätze keiner grundsätzlichen Präsupposition. Stattdessen scheint es sich bei weil um einen neutralen Konnektor zu handeln, der sowohl diskursneue als auch präsupponierte Propositionen einleiten kann, wohingegen die Subjunktion $d a$ lexikalisch als Einleitung von präsupponierten Kausalsätzen spezifiziert ist.

Da weil-Sätze grundsätzlich auch [-präsupponiert] sein können, ist eine Verwendung als Teil der Hauptstruktur anders als bei den präsupponierten Temporalsätzen nicht ausgeschlossen. Allerdings handelt es sich bei dem Merkmal [-präsupponiert] um kein hinreichendes, sondern nur um ein notwendiges Kriterium für [+at-issue]Inhalte, was das Beispiel der appositiven Relativsätze belegt, die zwar immer [-präsupponiert] sind, aber dennoch nie Teil der Hauptstruktur sein können. Nun stellt sich die Frage, weshalb die [-präsupponierten]-weil-Sätze, nicht aber die ebenfalls [-präsupponierten] appositiven Relativsätze [+at-issue]-Inhalt ausdrücken können. Eine mögliche Beantwortung dieser Frage könnte mit der grundlegenden Funktion von Kausalsätzen und appositiven Relativsätzen zusammenhängen. So besteht die grundlegende Funktion eines appositiven Relativsatzes ja gerade in der informationsstrukturellen Abwertung des in ihm ausgedrückten Inhalts im Vergleich zu selbstständigen Strukturen. (vgl. dazu u.a. Brandt 1996 und Hartmann 1984, 313f). Ein appositiver Relativsatz liefert eine zusätzliche, zur Identifikation des Bezugsreferenten nicht notwendige Information, darüber hinaus führt er aber keine zusätzliche semantische Relation, wie beispielsweise Kausalität oder Konzessivität, ein. Da nun aber die spezifische Funktion der Form des appositiven Relativsatzes in der infor- 
mationsstrukturellen Abwertung besteht, ist es nur logisch, dass appositive Relativsätze nicht zum Ausdruck von [+at-issue]-Inhalten geeignet sind. Dagegen ist die primäre Funktion eines Kausalsatzes keine informationsstrukturelle, vielmehr soll eine Begründung angeführt werden. Der in einer Begründung ausgedrückte Sachverhalt kann aber Teil der Haupt- oder der Nebenstruktur sein.

Dass weil-Sätze grundsätzlich auch ohne ihren Bezugssatz [+at-issue]-Inhalte enthalten können, wurde bereits in Abschnitt 2.2.3 gezeigt. In dieser Verwendung können sie dann auch mit V2-Stellung gebildet werden. Dass weil-Sätze anders als Temporalsätze [+at-issue]-Inhalt ausdrücken und V2-Stellung aufweisen können, kann demnach zumindest teilweise auf den grundlegenden funktionalen Unterschied zwischen weil auf der einen und temporalen Subjunktionen auf der anderen Seite zurückgeführt werden. Da temporale Subjunktionen als einstellige Ausdrücke als Quantorrestriktionen fungieren, unterliegen sie immer einer Existenzpräsupposition. Dies trifft dagegen nicht auf weil-Sätze zu, die als relationale Ausdrücke mit einer noch ungesättigten Argumentstelle keinen Quantor restringieren können. Um als temporaler Anker für den Hauptsatz zu fungieren, muss der in einem Temporalsatz ausgedrückte Sachverhalt zudem zum Äußerungszeitpunkt bereits gegeben und zeitlich fixiert sein. Auch dies trifft nicht auf Kausalsätze zu, da ein Sachverhalt nicht diskursverankert sein muss, um als Begründung für einen anderen Sachverhalt angeführt werden zu können. Wir können festhalten, dass keiner der Gründe, weswegen Temporalsätze immer präsupponiert sind, auf Kaulsätze zutrifft. Zudem liegt ihre Funktion anders als bei appositiven Relativsätzen nicht in der Herstellung einer informationsstrukturellen Hierarchie, weswegen Kausalsätze grundsätzlich dazu geeignet sind, [+at-issue]-Inhalt auszudrücken.

\subsection{Zusammenfassung des Kapitels}

In diesem Kapitel wurde der semantische Beitrag von temporalen Subjunktionen und dem Kaulsalkonnektor weil untersucht. Es wurde aufbauend auf dem Ansatz von Johnston (1994) und Zifonun et al. (1997) gezeigt, dass temporale Subjunktionen als einstellige Operatoren ihr IP-Komplement auf ein Zeitintervall i abbilden, welches immer die Domäne eines overten oder koverten Quantor restringiert. Der Bezugssatz bildet den Nukleus dieses Quantors und wird bezogen auf i interpretiert, wodurch die temporale Relation zwischen den beiden Teilsätzen zustande kommt. Ein komplexes Satzgefüge bestehend aus Haupt- und Temporalsatz wird meistens so interpretiert, dass die beiden ausgedrückten Ereignisse zeitlich nah beieinander liegen, was bedeutet, dass der Hauptsatz am linken bzw. rechten Rand des Intervalls verortet wird. Diese Beobachtung wurde in Abschnitt 9.1.2 als skalare Implikatur analysiert.

Als Quantorrestriktionen unterliegen Temporalsätze einer Existenzpräsupposition, womit in Abschnitt 9.1.3 der präsupponierte Status von Temporalsätzen begründet wurde. Es wurde zudem argumentiert, dass die durch einen Temporalsatz ausgelöste Präsupposition auch pragmatisch abgeleitet werden kann. Die Tatsache, dass Tem- 
poralsätze immer präsupponiert sind, kann demnach direkt aus ihrem spezifischen semantischen Beitrag abgeleitet werden. Da Präsuppositionen eine Untermenge der [-at-issue]-Inhalte bilden (vgl. Abschnitt 4), können Temporalsätze nicht dazu verwendet werden, ohne ihren Bezugssatz die aktuelle Q zu beantworten und demnach auch keine V2-Stellung aufweisen. In Abschnitt 9.1.3 wurde außerdem die durch einen Temporalsatz ausgelöste Präsupposition in SDRT implementiert. Dabei generiert der präsupponierte Inhalt eine separate Struktur, welche immer über eine unspezifizierte Diskursrelation $\mathrm{R}$ an ein direktes oder indirektes Antezedens angebunden werden muss. Es konnte gezeigt werden, dass die Äußerung dagegen nicht wohlgeformt ist, wenn eine solche Diskursanbindung scheitert.

Anders als temporale Subjunktionen führt weil eine zweistellige Kausalrelation zwischen Haupt- und Nebensatz ein. Es wurde argumentiert, dass weil-Sätze, die nicht als Quantorrestriktionen fungieren können, keiner Existenzpräsupposition unterliegen und auch aus pragmatischer Sicht nicht präsupponiert sein müssen. Da sie anders als appositive Relativsätze auch nicht der Herstellung einer informationsstrukturellen Abwertung dienen, sind Kausalsätze prinzipiell dazu geeignet, [+at-issue]-Inhalt auszudrücken. Der diskurssemantische Unterschied zwischen Temporal- und Kausalsätzen kann daher auf eine tieferliegende Unterscheidung zurückgeführt werden. 


\section{Schlussbemerkung}

In der neueren diskurssemantischen Forschung wird davon ausgegangen, dass jedem Text eine implizite oder explizite Frage zugrunde liegt, auf deren Beantwortung alle Äußerungen abzielen (vgl. u.a. Beaver/Clark 2008, Büring 2003, Büring 2007, Klein/von Stutterheim 1992, Onea 2011, Roberts 1996, Simons et al. 2011, von Stutterheim 1989 und von Stutterheim/Klein 1989). Die Grundannahmen eines solchen fragebasierten Diskursmodells wurden ausführlich zu Beginn der Arbeit diskutiert, wobei sich gezeigt hat, dass die jeweils relevante QUD sowohl den formalen als auch den inhaltlichen Diskursaufbau steuert, indem sie zum einen bestimmt, was gesagt wird, und zum anderen die Form des Gesagten beeinflusst. Äußerungen können darauf abzielen, die aktuell relevante QUD zu beantworten, dann sind sie Teil der Hauptstruktur und drücken [+at-issue]-Inhalt aus, oder sie liefern zusätzliche Hintergrundinformationen und sind demzufolge [-at-issue]. In einem Diskurs wird anhand sprachlicher Markierungen wie Fokusintonation oder Definitheit zudem angezeigt, ob eine Äußerung oder ein Äußerungsteil relevanten Inhalt bezogen auf die QUD ausdrückt.

In der germanistischen Forschung wurde lange Zeit davon ausgegangen, dass auch die Verwendung von Nebensätzen mit einer textstrukturellen Abwertung verbunden ist (vgl. beispielsweise Bartsch 1978, Hartmann 1984, Posner 1972 und von Stutterheim 1989, 168). Brandt (1989, 1996), Holler (2009) und Simons (2007) beobachten jedoch, dass auch abhängige Sätze ohne ihren Bezugssatz die QUD beantworten können. Ihre Untersuchungen beschränken sich allerdings nur auf wenige Nebensatztypen, zudem bleibt ungeklärt, inwiefern der textstrukturelle Status von Nebensätzen deren Form und ihre anderen (diskurs)semantische Eigenschaften beeinflusst. Da das Verhalten von abhängigen Sätzen in Bezug auf die diskurssemantische Textstrukturierung in einem fragebasierten Diskursmodell bislang nur unzureichend erforscht war, bestand das Ziel der vorliegenden Arbeit darin, diese Lücke zu schließen. Gleichzeitig wurde untersucht, welche anderen syntaktischen und semantischen Eigenschaften der jeweiligen Nebensatztypen von ihrem diskursstrukturellen Status abhängen.

Im Detail wurden in der vorliegenden Arbeit die folgenden Ergebnisse erzielt:

In Teil I konnte gezeigt werden, dass zwei Gruppen von Nebensätzen voneinander zu unterscheiden sind. Während einige Nebensätze genau wie selbstständige Sätze ohne ihren Bezugssatz [+at-issue]-Inhalt ausdrücken können, sind andere grundsätzlich nicht dazu geeignet und gehören entweder zur Nebenstruktur oder zielen lediglich als Konstituente ihres Matrixsatzes mit diesem zusammen auf die Beantwortung der jeweils relevanten QUD ab. Dabei wurde folgende Klassifikation vorgeschlagen: 
Gruppe 1:

Abhängige Sätze, die auch ohne ihren Bezugssatz [+at-issue]-Inhalt ausdrücken können:

Komplementsätze von Brücken- und semifaktiven Verben, weil-Sätze, restriktive Relativsätze mit einem indefiniten, spezifischen Antezedens.

\section{Gruppe 2:}

Abhängige Sätze, die nich dazu geeignet sind, ohne ihren Bezugssatz [+at-issue]-Inhalt auszudrücken:

Komplementsätze von faktiven Verben, Temporalsätze, appositive Relativsätze, restriktive Relativsätze mit einem indefiniten nicht-spezifischen Antezedens, restriktive Relativsätze mit einem definiten Antezedens.

In Kapitel 3 wurde diese Einteilung zusätzlich bestätigt, indem gezeigt wurde, dass die Nebensätze aus Gruppe 2 zentrale Eigenschaften aufweisen, welche nach Roberts et al. (2009) und Simons et al. (2011) [-at-issue]-Eigenschaften auszeichnen. Es hat sich herausgestellt, dass die Nebensätze aus Gruppe 2 projizieren, wenn sie im Skopus eines sonst implikationstilgenden Operators stehen, wohingegen die Nebensätze aus Gruppe 1 nur in Kontexten, in denen sie als Teil der Nebenstruktur verwendet werden, unter Einbettung überleben. Die in Kapitel rein introspektiv vorgenommene Klassifikation der Nebensätze in die beiden Gruppen konnte auch anhand einer Fragebogenstudie empirisch bestätigt werden. Ausgangspunkt für die in Kapitel 3.2 vorgestellte Fragebogenstudie war die Annahme von Posner (1972), Roberts et al. (2009) und Simons et al. (2011), derzufolge [-at-issue]-Inferenzen nicht sensibel für einen direkten Widerspruch mit Nein sind. Die Ergebnisse der empirischen Untersuchung zeigten eindeutig, dass dem Inhalt eines Nebensatzes aus Gruppe 2 nicht direkt widersprochen werden kann, was ebenfalls belegt, dass die Sätze immer [-at-issue]-Inhalt ausdrücken. Bezogen auf die Nebensätze aus Gruppe 2 zeigten die Ergebnisse der Fragebogenstudie dagegen, dass diese Sätze mit einer unterschiedlich starken Präferenz als [+at-issue] interpretiert werden.

Sowohl Projektion als auch direkte Widerspruchsstrategien werden traditionell als Präsuppositionstests angesehen. Durch die Ergebnisse der vorliegenden Arbeit konnte allerdings die in Roberts et al. (2009) und Simons et al. (2011) vertretene Auffassung, derzufolge mithilfe der beiden Tests At-issueness ermittelt wird, bestätigt werden. Das Verhältnis von Präsuppositionen und dem Konzept der At-issueness wurde in Kapitel 4 genauer beleuchtet. Wie andere [-at-issue]-Inferenzen projizieren Präsuppositionen unter Einbettung und sind nicht sensibel für direkte Widerspruchsstrategien. Es konnte jedoch gezeigt werden, dass nicht alle Bedeutungsaspekte, die [-at-issue]-Inhalt ausdrücken, auch gleichzeitig präsupponiert sind. So gehören beispielsweise appositive Relativsätze immer zur Nebenstruktur, können jedoch nie eine im Diskurskontext bereits gegebene Information ausdrücken, was zeigt, dass sie nie präsupponiert sind. Aus diesem Grund wurde argumentiert, dass es sich bei Präsuppositionen um eine Untermenge von [-at-issue]-Inferenzen handelt, wie in Abbildung 10.1 dargestellt. 


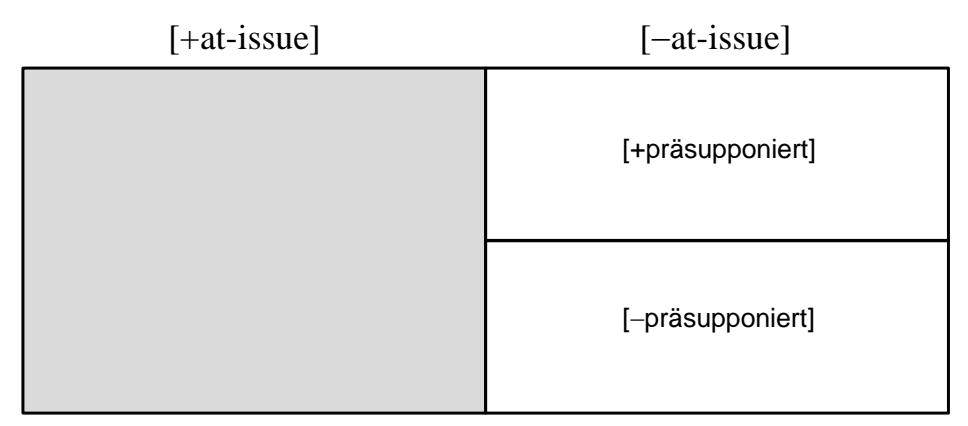

Abbildung 10.1.: Präsuppositionen und At-issueness

Der Unterschied zwischen Präsuppositionen und nicht-präsupponierten Bedeutungsaspekten wurde in SDRT modelliert. Dabei wurde vorgeschlagen, dass Präsuppositionen typischerweise über die Diskursrelation $\mathrm{R}=$ Coreference an ein koreferentes Antezedens angebunden werden. Bei diesem Antezedens kann es sich um eine Variable für ein Individuum oder aber um eine Variable für ein Ereignis handeln. Wird eine Präsupposition über eine andere Diskursrelation als Coreference angebunden, handelt es sich um einen Fall von Bridging. Bezogen auf nicht-präsupponierte Inhalte (wie beispielsweise V2-Nebensätze, appositive Relativsätze oder indefinite NPn) konnte dagegen gezeigt werden, dass diese grundsätzlich nicht über die Diskursrelation $\mathrm{R}=$ Coreference mit einem Antezedens verknüpft werden können. Aufbauend auf dieser Beobachtung wurde dafür plädiert, nicht nur Präsuppositionen anhand spezifischer Eigenschaften zu charakterisieren, sondern auch Inhalte, die keine präsupponierte Lesart zulassen, über ihr Unvermögen über $\mathrm{R}=$ Coreference angebunden zu werden, zu definieren.

In Teil II der vorliegenden Arbeit wurde das Auftreten von V2-Stellung in abhängigen Sätzen des Deutschen genauer untersucht. Die Tatsache, dass V2-Stellung nur in manchen Nebensatztypen möglich ist, während sie in anderen Nebensätzen grundsätzlich ausgeschlossen ist, verlangt nach einer Klärung der zugrundeliegenden Lizenzierungsbedingungen. In zahlreichen bisherigen Arbeiten wurde die von Hooper/Thompson (1973) für das Englische entwickelte Assertionshypothese, derzufolge nur assertierte Nebensätze Wurzelphänomene aufweisen können, auf das Auftreten von V2Stellung in abhängigen Sätzen im Deutschen übertragen. In Abschnitt 7.1 wurde jedoch gezeigt, dass dies mit zwei Problemen verbunden ist: Zum einen existieren Nebensätze, die Ähnlichkeiten zu Assertionen aufweisen, ohne deswegen mit V2Stellung gebildet werden zu können, zum anderen besitzen nicht alle V2-Nebensätze ein assertives Potential. Aus diesem Grund wurde in Abschnitt 7.2 ein alternativer Ansatz vorgestellt. Genauer gesagt wurde argumentiert, dass im Deutschen nur Nebensätze, die [+at-issue]-Inhalt ausdrücken, V2-Stellung aufweisen können und es sich bei V2-Stellung in abhängigen Strukturen somit um eine optionale Markierung für At-issueness handelt. Typischerweise sind Assertionen auch [+at-issue] und umgekehrt, die beiden Eigenschaften sind jedoch nicht immer deckungsgleich. Um assertierte von [+at-issue]-Inhalten abzugrenzen, wurden unterschiedliche Tests ver- 
wendet. Es wurde davon ausgegangen, dass es sich bei einer Äußerung U um eine Assertion handelt, wenn U:

- nicht in den Matrixsprechakt integriert werden kann,

- nicht in die Matrix-FHG integriert werden kann,

- MPn, Einstellungsadverbien und Performanzanzeiger aufweisen kann,

- durch den Sprecher selbst nicht direkt zurückgenommen werden kann.

Eine Proposition p ist dagegen [+at-issue], wenn:

- die Auflösung von ?p zu einer Reduktion des durch die aktuelle QUD eröffneten Alternativenraums führt,

- p unter Einbettung unter einen inferenztilgenden Operator nicht projiziert,

- $\mathrm{p}$ sensibel für einen direkten Widerspruch durch andere Diskursteilnehmer ist.

Eine genaue Untersuchung von abhängigen V2-Strukturen im Deutschen hat ergeben, dass diese nicht immer alle relevanten Merkmale einer Assertion aufweisen, wohingegen sie immer [+at-issue]-Inhalt ausdrücken.

Die Analyse wurde in Abschnitt 7.3 auf weitere Nebensätze des Deutschen und des Englischen ausgeweitet. Es hat sich gezeigt, dass die Vorhersage der At-issuenessHypothese bezogen auf obwohl-Sätze mit V2-Stellung zutreffend ist, da diese immer eine Unterfrage der jeweils relevanten QUD beantworten. Problematisch in Bezug auf die At-issueness-Hypothese verhalten sich dagegen Konditionalsätze, da diese prinzipiell dazu verwendet werden können, ohne ihren Bezugssatz [+at-issue]-Inhalt auszudrücken, gleichzeitig aber, wenn sie mit wenn eingeleitet sind, nie V2-Stellung aufweisen können. Diesbezüglich wurde vorgeschlagen, dass im konditionalen Bereich ähnlich wie im kausalen eine lexikalische Ausdifferenzierung vorliegt - während wenn genau wie da immer VL-Sätze einleitet, können Sätze mit vorausgesetzt oder angenommen ähnlich wie weil-Sätze auch V2-Stellung aufweisen.

Auch VL-Nebensätze können wie abhängige V2-Sätze desintegriert auftreten und verhalten sich dann in vielen Punkten wie V2-Nebensätze. In Abschnitt 7.3.3 konnte jedoch gezeigt werden, dass Desintegration und At-issueness nicht grundsätzlich korrelieren - während ein V2-Nebensatz immer Teil der Hauptstruktur ist, trifft dies nicht im gleichen Maße auf desintegrierte VL-Nebensätze, umgekehrt können auch kanonische, integrierte Nebensätze [+at-issue]-Inhalt ausdrücken.

Ein kurzer Exkurs zum Auftreten von Topikalisierungen und Linksversetzungen in englischen Nebensätzen hat dagegen gezeigt, dass diese in allen Sätzen, die [-präsupponiert] sind, auftreten. Somit handelt es sich bei den Nebensätzen, die im Deutschen V2-Stellung aufweisen können, um eine Untermenge der Kontexte, welche im Englischen Wurzelphänomene lizenzieren. 
Dass bezogen auf die Fähigkeit, [+at-issue]-Inhalt auszudrücken und somit auch V2Stellung aufweisen zu können, zwei Arten an Nebensätzen zu unterscheiden sind, wurde in Teil III am Beispiel der temporalen und kausalen Adverbialsätze auf einen tieferliegenden Unterschied zurückgeführt. Aufbauend auf den Ergebnissen von Johnston (1994) zum Englischen wurde gezeigt, dass sich temporale Subjunktionen in der Art ihres semantischen Beitrags grundlegend von kausalen unterscheiden. Da sich Temporalsätze in negierten, quantifizierten und in Kopulakonstruktionen nicht wie relationale Ausdrücke verhalten, wurde geschlossen, dass temporale Subjunktionen einstellig sind und ihr IP-Komplement auf ein Zeitintervall i abbilden. Das kausale weil führt dagegen eine zweistellige Relation wie in (393-a) ein. Zudem wurde argumentiert, dass das durch einen Temporalsatz denotierte Intervall ähnlich wie ein Konditionalsatz immer die Domäne eines overten oder koverten Quantors restringiert, in dessen Nukleus der Bezugssatz steht, wie in (393-b) für einen bevor-Satz vereinfacht dargestellt.

$$
\begin{array}{ll}
\text { a. } & \text { weil }(\mathrm{p}, \mathrm{q}) \\
\text { b. } & \exists / \forall\{\operatorname{bevor}(\mathrm{p})\}[\mathrm{q}]
\end{array}
$$

Es wurde somit eine neue Analyse des semantischen Beitrags von temporalen Subjunktionen im Deutschen dargelegt, welche das Verhalten von Temporalsätzen in quantifizierten und negierten Strukturen besser erfasst als die traditionelle Beschreibung von temporalen Subjuntkionen als relationale Ausdrücke. Basierend auf diesem neuen Ansatz wurde auch die Tatsache, dass Temporalsätze nie [+at-issue]Inhalt ausdrücken können, abgeleitet. Als Quantorrestriktionen unterliegen Temporalsätze immer einer Existenzpräsupposition, welche im Rahmen von SDRT nach Asher/Lascarides (2003) modelliert wurde. Da Präsuppositionen Untermenge von [-at-issue]-Inferenzen bilden, folgt aus dem präsupponierten Status von Temporalsätzen, dass sie zur Beantwortung der aktuell relevanten QUD nicht geeignet sind und demnach auch nie V2-Stellung aufweisen können.

Die vorliegende Arbeit zeigt, dass anhand des diskurssemantischen Konzepts der Atissueness zentrale strukturelle und funktionale Eigenschaften von abhängigen Sätzen wie beispielsweise Projektion unter Einbettung oder das Auftreten von V2-Stellung abgeleitet werden können. Insbesondere bezogen auf das Phänomen der V2-Stellung in abhängigen Strukturen, welches traditionell unter Rückgriff auf die Begriffe Assertion und Präsupposition erklärt wird, wurde somit eine neue Sichtweise dargelegt. Tabelle 10.1 fasst noch einmal alle in dieser Arbeit diskutierten formalen und (diskurs)semantischen Eigenschaften der relevanten acht Nebensätze zusammen.

Aus der in dieser Arbeit vorgestellten Studie ergeben sich zahlreiche offene Fragen und Anknüpfungspunkte für zukünftige Untersuchungen:

- Es wurden nur ausgesuchte Nebensatztypen betrachtet, eine Klassifikation weiterer Nebensätze steht dabei noch aus. Insbesondere ist dabei zu überprüfen, ob die Korrelation zwischen At-issueness und V2-Stellung auch bei den hier nicht untersuchten Nebensatztypen zutreffend ist. 


\begin{tabular}{|l||c|c|c|c|c|c|}
\hline Nebensatztyp & at-issue & Proj. & Wid. & präs. & V2 & Top. Engl. \\
\hline \hline $\begin{array}{l}\text { Komplementsatz, } \\
\text { Brückenverb }\end{array}$ & $+/-$ & $+/-$ & $+/-$ & $+/-$ & $+/-$ & $+/-$ \\
\hline $\begin{array}{l}\text { Semifaktiver Komple- } \\
\text { mentsatz }\end{array}$ & $+/-$ & $+/-$ & $+/-$ & $+/-$ & $+/-$ & $+/-$ \\
\hline Faktiver Komplementsatz & - & + & - & + & - & - \\
\hline Temporalsatz & - & + & - & + & - & - \\
\hline Adverbialsatz mit weil & $+/-$ & $+/-$ & $+/-$ & $+/-$ & $+/-$ & $+/-$ \\
\hline Appositiver Relativsatz & - & + & - & - & - & + \\
\hline $\begin{array}{l}\text { Restriktiver Relativsatz, } \\
\text { definites Antezedens }\end{array}$ & - & + & - & + & - & - \\
\hline $\begin{array}{l}\text { Restriktiver Relativsatz, } \\
\text { indefinites Antezedens }\end{array}$ & $+/-$ & $+/-$ & $+/-$ & $+/-$ & $+/-$ & $+/-$ \\
\hline
\end{tabular}

Tabelle 10.1.: Die diskutierten strukturellen und funktionalen Eigenschaften im Überblick

- In der Untersuchung von abhängigen V2-Strukturen in Teil II wurden Kontexte, in denen Nebensätze grundsätzlich nicht mit V2-Stellung gebildet werden können, ausgeklammert. Beispielsweise können V2-Nebensätze nie im Skopus der Matrixnegation oder im Vorfeld stehen (für einen systematischen Überblick über die inkompatiblen Kontexte vgl. Antomo/Steinbach 2010 und Holler 2008). Von der Forschung zu klären ist die Frage, ob die Blockerierung von V2-Stellung in diesen Kontexten oder zumindest in einigen dieser Kontexte ebenfalls über das Konzept der At-issueness abgeleitet werden kann.

- Am Beispiel von weil- und Temporalsätzen wurde der Frage auf den Grund gegegangen, aus welchem Grund manche Nebensätze [+at-issue]-Inhalt ausdrücken können, andere dagegen nicht. Eine solche Untersuchung ist auch noch für die weiteren Nebensatztypen wünschenswert.

- Das Projektionsverhalten von abhängigen Sätzen bedarf noch einer empirischen Überprüfung.

- Aufgrund ihres Verhaltens in negierten und quantifizierten Strukturen wurde geschlossen, dass temporale Subjunktionen semantisch einstellig sind, das kausale weil dagegen zweistellig. Einiges spricht dafür, dass es sich mit temporalen und kausalen Präpositionen ähnlich verhält, was von der Forschung noch zu überprüfen ist. 
A. Testsätze 


\section{A. Testsätze}

\section{Kondition 1: Komplementsatz, Brückenverb}

(1) A: Philipp hat behauptet, dass Sabine raucht.

B: Nein!

Raucht Sabine?

(2) A: Lea findet, dass sie zu dick ist.

B: Nein!

Ist Lea zu dick?

(3) A: Andreas hat erzählt, dass die Schule renoviert wird.

B: Nein!

Wird die Schule renoviert?

(4) A: Michael glaubt, dass er geblitzt wurde.

B: Nein!

Wurde Michael geblitzt?

(5) A: Anke hat gesagt, dass der neue Lehrer nett ist.

B: Nein!

Ist der neue Lehrer nett?

(6) A: Katrin vermutet, dass sie zu wenig gelernt hat.

B: Nein!

Hat Katrin zu wenig gelernt?

\section{Kondition 2: Semifaktiver Komplementsatz}

(7) A: Peter hat entdeckt, dass er sich verrechnet hat.

B: Nein!

Hat sich Peter verrechnet?

(8) A: Jenny hat herausgefunden, dass Felix gelogen hat.

B: Nein!

Hat Felix gelogen?

(9) A: Fabian hat erfahren, dass er die Klausur bestanden hat.

B: Nein!

Hat Fabian die Klausur bestanden?

(10) A: Birgit erkennt, dass Jürgen ein Betrüger ist.

B: Nein!

Ist Jürgen ein Betrüger? 
(11) A: Silke bemerkt, dass sie zugenommen hat.

B: Nein!

Hat Silke zugenommen?

(12) A: Benjamin stellt fest, dass Susanne unschuldig ist.

B: Nein!

Ist Susanne unschuldig?

\section{Kondition 3: Faktiver Komplementsatz}

(13) A: Fred ignoriert, dass seine Frau fremdgeht.

B: Nein!

Geht Freds Frau fremd?

(14) A: Maria bereut, dass sie Jonas verlassen hat.

B: Nein!

Hat Maria Jonas verlassen?

(15) A: Isabel hat vernachlässigt, dass die Mietpreise gestiegen sind.

B: Nein!

Sind die Mietpreise gestiegen?

(16) A: Theresa hat beachtet, dass die Sitzung verschoben wurde.

B: Nein!

Wurde die Sitzung verschoben?

(17) A: Markus bedauert, dass Lena krank ist.

B: Nein!

Ist Lena krank?

(18) A: Christoph hat sich gefreut, dass ihn seine Freunde im Krankenhaus besucht haben.

B: Nein!

Haben Christophs Freunde ihn im Krankenhaus besucht?

\section{Kondition 4: Temporalsatz}

(19) A: Petra war einkaufen, bevor sie die Kinder abgeholt hat.

B: Nein!

Hat Petra die Kinder abgeholt?

(20) A: Marlene war in der Vorlesung, als Tim einen Autounfall hatte.

B: Nein!

Hatte Tim einen Autounfall? 


\section{A. Testsätze}

(21) A: Anna ging ins Kino, nachdem sie die Steuererklärung abgeschickt hatte.

B: Nein!

Hat Anna die Steuererklärung abgeschickt?

(22) A: Klaus hat alle Testberichte gelesen, bevor er einen neuen Computer gekauft hat.

B: Nein!

Hat Klaus einen neuen Computer gekauft?

(23) A: Georg hatte Mittagspause, als im Park eine Leiche gefunden wurde.

B: Nein!

Wurde im Park eine Leiche gefunden?

(24) A: Lukas fuhr ins Schwimmbad, nachdem Tina das Auto repariert hatte.

B: Nein!

Hat Tina das Auto repariert?

\section{Kondition 5: Weil-Satz}

(25) A: Nadine verkauft ihr Auto, weil es kaputt ist.

B: Nein!

Ist Nadines Auto kaputt?

(26) A: Annika fliegt nach China, weil sie dort arbeiten muss.

B: Nein!

Muss Annika in China arbeiten?

(27) A: Walter ist zu spät gekommen, weil die Wilhelmstraße gesperrt ist.

B: Nein!

Ist die Wilhelmstraße gesperrt?

(28) A: Nathalie hat gekündigt, weil sie im Lotto gewonnen hat.

B: Nein!

Hat Nathalie im Lotto gewonnen?

(29) A: Anton fährt mit dem Fahrrad, weil die Benzinpreise gestiegen sind.

B: Nein!

Sind die Benzinpreise gestiegen?

(30) A: Alexander hat seine Frau abgeholt, weil die Busfahrer streiken.

B: Nein!

Streiken die Busfahrer? 


\section{Kondition 6: Appositiver Relativsatz}

(31) A: Paul, der Frank verprügelt hat, wohnt in Göttingen.

B: Nein!

Wurde Frank von Paul verprügelt?

(32) A: Monika, die übrigens Physik studiert hat, war auf der Party.

B: Nein!

Hat Monika Physik studiert?

(33) A: Emma, die übrigens schwanger ist, kann super kochen.

B: Nein!

Ist Emma schwanger?

(34) A: Elisabeth, die bei der Deutschen Bank arbeitet, wurde von der Steuerfahndung angerufen. B: Nein!

Arbeitet Elisabeth bei der Deutschen Bank?

(35) A: Thomas, der gerne Motorrad fährt, hatte gestern einen Unfall.

B: Nein!

Fährt Thomas gerne Motorrad?

(36) A: Max, der übrigens mit Antje verheiratet war, wurde befördert.

B: Nein!

War Max mit Antje verheiratet?

\section{Kondition 7: Restriktiver Relativsatz, definites Antezedens}

(37) A: Christina kennt den Mann, der ihr Auto gestohlen hat.

B: Nein!

Wurde Christinas Auto von einem Mann gestohlen?

(38) A: Daniel mag die Frau, die ihn in der Mensa angesprochen hat.

B: Nein!

Wurde Daniel in der Mensa von einer Frau angesprochen?

(39) A: Verena liest den Artikel, den Niels über Afghanistan geschrieben hat.

B: Nein!

Hat Niels einen Artikel über Afghanistan geschrieben?

(40) A: Oliver hat den Hund gefüttert, den Barbara aus dem Tierheim mitgebracht hat.

B: Nein!

Hat Barbara einen Hund aus dem Tierheim mitgebracht? 


\section{A. Testsätze}

(41) A: Michaela hat den Kuchen gegessen, den Klara auf dem Gemeindebasar gekauft hat. B: Nein!

Hat Klara auf dem Gemeindebasar Kuchen gekauft?

(42) A: David hat die Vase bezahlt, die er im Museum umgestoßen hat.

B: Nein!

Hat David im Museum eine Vase umgestoßen?

Kondition 8: Restriktiver Relativsatz, indefinites Antezedens

(43) A: Jörg liest ein Buch, das ihm seine Lehrerin empfohlen hat.

B: Nein!

Hat Jörgs Lehrerin ihm ein Buch empfohlen?

(44) A: Wiebke guckt eine DVD, die ihr Tobias geliehen hat.

B: Nein!

Hat Tobias Wiebke eine DVD geliehen?

(45) A: Angela fährt einen Porsche, der Doris gehört.

B: Nein!

Gehört Doris ein Porsche?

(46) A: Konstantin sucht eine Frau, die ihn eingeparkt hat.

B: Nein!

Wurde Konstantin von einer Frau eingeparkt?

(47) A: Caroline hat eine Kette ersteigert, die Martin in Südafrika gefunden hat.

B: Nein!

Hat Martin in Südafrika eine Kette gefunden?

(48) A: Rüdiger hat ein abstraktes Bild gewonnen, das Anja gemalt hat.

B: Nein!

Hat Anja ein abstraktes Bild gemalt?

\section{Kontrollsätze:}

(49) A: Patricks Frau hat behauptet, dass das Sportfest verschoben wird.

B: Nein!

Ist Patrick verheiratet?

(50) A: Lauras Tochter gibt Nachhilfeunterricht, weil sie sich etwas Geld dazu verdienen will.

B: Nein!

Hat Laura Kinder? 
(51) A: Der Gärtner vom Präsidenten fuhr in den Baumarkt, um Blumenerde zu kaufen. B: Nein!

Hat der Präsident einen Gärtner?

(52) A: Die Schwester vom Pfarrer, die in München lebt, ist Lehrerin.

B: Nein!

Hat der Pfarrer eine Schwester?

(53) A: Sophia, die jetzt übrigens kurze Haare hat, kommt aus Polen.

B: Nein!

Kommt Sophia aus Polen?

(54) A: Florian, der seit neustem geschieden ist, fliegt über Weihnachten nach Mexiko.

B: Nein!

Fliegt Florian über Weihnachten nach Mexiko?

(55) A: Der Richter, der übrigens bald in Rente geht, hat Mathilda verurteilt.

B: Nein!

Hat der Richter Mathilda verurteilt?

(56) A: Sarah, die gerne taucht, ist Zahnärztin.

B: Nein!

Ist Sarah Zahnärztin?

(57) A: Martha ist zum Zahnarzt gefahren, nachdem die Konferenz beendet war.

B: Nein!

Hat Martha einen Hund?

(58) A: Leon findet es schade, dass es im Urlaub viel geregnet hat.

B: Nein!

Ist Leon Anwalt?

(59) A: Der Koch bestellt Rotkohl, weil bald die Weihnachtssaison beginnt.

B: Nein!

Ist der Koch Raucher?

(60) A: Sebastian vermutet, dass Marcel keinen Parkplatz gefunden hat.

B: Nein!

Ist Sebastian Vegetarier? 
A. Testsätze 


\section{Literaturverzeichnis}

Altmann, Hans (1993): Satzmodus. In: Jacobs, Joachim et al. (Hg.), Syntax. Ein internationales Handbuch zeitgenössischer Forschung, Berlin, New York: de Gruyter, 1006-1029.

Altmann, Hans (1997): Verbstellungsprobleme bei subordinierten Sätzen in der deutschen Sprache. In: Dürscheid, Christa et al. (Hg.), Sprache im Fokus. Festschrift für Heinz Vater zum 65. Geburtstag, Tübingen: Niemeyer, 69-84.

Amaral, Patrícia et al. (2011): Experimental evidence on the distinction between foregrounded and backgrounded meaning. In: Proceedings of ESSLLI 2011 Workshop on Projective Meaning.

Andersson, L. (1975): Form and function of subordinate clauses. Dissertation, Göteborg University, Göteborg.

Antomo, Mailin (2012a): Interpreting Embedded Verb Second. Causal modifiers in German. In: Constantinescu, Camelia et al. (Hg.), Proceedings of ConSOLE XVII, $27-51$.

Antomo, Mailin (2012b): Projective Meaning and the licensing of Embedded Root Phenomena. In: Boone, Enrico et al. (Hg.), Proceedings of Console XIX, 1-23.

Antomo, Mailin/Steinbach, Markus (2010): Desintegration und Interpretation: WeilV2-Sätze an der Schnittstelle zwischen Syntax, Semantik und Pragmatik. In: Zeitschrift für Sprachwissenschaft 29.1, 1-37.

Antomo, Mailin/Steinbach, Markus (2013): Zur Semantik von Konzessivsätzen mit obwohl. In: Linguistische Berichte 236, 427-453.

Asher, Nicholas/Lascarides, Alex (1998a): Bridging. In: Journal of Semantics 15.1, 83-113.

Asher, Nicholas/Lascarides, Alex (1998b): The semantics and pragmatics of presupposition. In: Journal of Semantics 15.2, 239-299.

Asher, Nicholas/Lascarides, Alex (2003): Logics of conversation. Cambridge, New York: Cambridge University Press.

Auer, Peter (1998): Zwischen Parataxe und Hypotaxe: 'abhängige Hauptsätze' im gesprochenen und geschriebenen Deutsch. In: Zeitschrift für Germanistische Linguistik 26, 284-307. 
Averintseva-Klisch, Maria/Volodina, Anna (2012): Causality and at-issueness: some insights from German subordinate connective $d a$. Handout, 34. DGfSJahrestagung, Frankfurt a. M. .

Axel-Tober, Katrin (2012): (Nicht-)kanonische Nebensätze im Deutschen. Synchrone und diachrone Aspekte., Linguistische Arbeiten, Band 542. Berlin, New York: Walter de Gruyter.

Bach, Kent (1999): The myth of conventional implicature. In: Linguisics and Philosophy $22,327-366$.

Ballweg, Joachim (1988): Die Semantik der deutschen Tempusformen. Düsseldorf: Schwann.

Barr, Dale J. et al. (2013): Random effects structure for confirmatory hypothesis testing: Keep it maximal. In: Journal of Memory and Language 68.3, 255-278.

Bartsch, Renate (1978): Satzreihung, Satzgefüge oder Adverbialkonstruktion? Über pragmatische und kontextuelle Unterschiede zwischen semantisch gleichwertigen Aussagen. In: Hartmann, Dietrich et al. (Hg.), Sprache in Gegenwart und Geschichte, Köln, Wien: Böhlau Verlag, 1-18.

Batliner, Anton et al. (1995): The Prosodic Marking of Phrase Boundaries: Expectations and Results. In: Ayuso, Antonio et al. (Hg.), Speech Recognition and Coding, Berlin Heidelberg: Springer, NATO ASI Series, Band 147, 325-328.

Bayer, Josef (2004): Decomposing the left periphery: dialectal and cross-linguistic evidence. In: Lohnstein, Horst/Trissler, Susanne (Hg.), The syntax and semantics of the left periphery, Berlin: de Gruyter, 59-95.

Beaver, David (2012): Antimatters. Handout, 34. DGfS-Jahrestagung, Frankfurt a.M.

Beaver, David/Clark, Brady (2008): Sense and sensitivity. How focus determines meaning. Oxford: Blackwell.

Beaver, David/Condoravdi, Cleo (2003): A uniform analysis of Before and After. In: Young, Rob/Zhou, Yuping (Hg.), Proceedings of SALT XIII, Cornell: CLC Publications, 37-54.

Beaver, David I. (2001): Presupposition and Assertion in Dynamic Semantics. Stanford: CSLI Publications.

Bentzen, Kristine (2009): Embedded root phenomena, assertion, presupposition, and main point of utterance, vortrag auf der Tagung "Root Phenomena", ZAS Berlin, 2.-4.09.2009. 
Bentzen, Kristine (2010): Exploring embedded main clause phenomena: The irrelevance of factivity and some challenges from V2 languages. In: Theoretical Linguistics $36.2 .3,163-172$.

Bentzen, Kristine et al. (2007): The Tromso guide to the force behind V2. In: Working Papers in Scandinavian Syntax 79, 93-118.

Bianchi, Valentina/Frascarelli, Mara (2009): Is topic a root phenomenon? In: Talk presented at the workshop Root Phenomena, ZAS Berlin .

Bierwisch, Manfred (1980): Semantic structure and illocutionary force. In: Searle, John (Hg.), Speech act theory and pragmatics, Dordrecht: Reidel, 1-36.

Blühdorn, Hardarik (2004a): Die Konjunktionen nachdem und bevor. In: Blühdorn, Hardarik et al. (Hg.), Brücken schlagen. Grundlagen der Konnektorensemantik, Berlin: de Gruyter, 185-211.

Blühdorn, Hardarik (2004b): Einleitung. In: Blühdorn, Hardarik et al. (Hg.), Brücken schlagen. Grundlagen der Konnektorensemantik, Berlin: de Gruyter, 125136.

Blühdorn, Hardarik (2006): Kausale Satzverknüpfungen im Deutschen. In: Pandaemonium Germanicum. Revista de Estudos Germanísticos, Sao Paulo, Band 10, $253-282$.

Blühdorn, Hardarik (2008): Epistemische Lesarten von Satzkonnektoren ? Wie sie zustande kommen und wie man sie erkennt. In: Pohl, Inge (Hg.), Semantik und Pragmatik-Schnittstellen, Frankfurt a. M.: Lang, 217-251.

Borst, Dieter (1985): Die affirmativen Modalpartikeln doch, ja und schon. Ihre Bedeutung, Funktion, Stellung und ihr Vorkommen. Tübingen: Niemeyer.

Brandner, Ellen (2004): Head movement in minimalism, and V2 and FORCE marking. In: Horst Lohnstein/Trissler, Susanne (Hg.), The syntax and semantics of the left periphery, Berlin: de Gruyter, 313-341.

Brandt, Margareta (1989): Zur Fokus-Hintergrund-Gliederung in komplexen Sätzen. In: Sprache und Pragmatik 13, 43-54.

Brandt, Margareta (1990): Weiterführende Nebensätze: Zu ihrer Syntax, Semantik und Pragmatik, Lunder germanistische Forschungen, Band 57. Stockholm: Almqvist \& Wiksell.

Brandt, Margareta (1996): Subordination und Parenthese als Mittel der Informationsstrukturierung in Texten. In: Motsch, Wolfgang (Hg.), Ebenen der Textstruktur. Sprachliche und kommunikative Prinzipien, Tübingen: Niemeyer, 211-240. 
Brandt, Margareta et al. (1992): Satztyp, Satzmodus und Illokution. In: Rosengren, Inger (Hg.), Satz und Illokution 1, Niemeyer, 1-90.

Breindl, Eva (2009): Fehler mit System und Fehler im System. In: Konopka, Marek/Strecker, Bruno (Hg.), Deutsche Grammatik - Regeln, Normen, Sprachgebrauch. Jahrbücher des IDS 2008, Berlin, New York: de Gruyter, 274-306.

Büring, Daniel (2003): On D-trees, beans and B-accents. In: Linguistics and Philosophy $26.5,511-545$.

Büring, Daniel (2006): Intonation und Informationsstruktur. In: Blühdorn, Hardarik et al. (Hg.), Text-Verstehen. Grammatik und darüber hinaus, Berlin, New York: de Gruyter, 144-163.

Büring, Daniel (2007): Intonation, semantics and information structure. In: Ramchand, Gillian/Reiss, Charles (Hg.), The Oxford Handbook of Linguistic Interfaces, Oxford University Press.

Burkhardt, Petra (2005): The syntax-discourse interface. Representing and interpreting dependencyA. Amsterdam, Philadelphia: John Benjamins.

Burkhardt, Petra (2006): Inferential bridging relations reveal distinct neural mechanisms: Evidence from event-related brain potentials. In: Brain and Language 98, $159-168$.

Buscha, Joachim (1989): Lexikon deutscher Konjunktionen. Leipzig: VEB.

Chierchia, Gennaro (1995): Dynamics of meaning. Anaphora, presupposition and the theory of grammar. Chicago, London: University of Chicago Press.

Chierchia, Gennaro/McConnell-Ginet, Sally (1990): Meaning and Grammar: An introduction to Semantics. Cambridge, Massachusetts: MIT Press.

Clark, Herbert (1975): Bridging. In: Schank, Roger C./Nash-Webber, Bonnie L. (Hg.), Theoretical issues in natural language processing, Association for Computing Machinery, 169-174.

Coniglio, Marco (2007): German modal particles in root and embedded clauses. In: Working Papers in Linguistics 17, 109-141.

Copley, Bridget (2009): The semantics of the future. New York: Routledge.

Cunha Lima, Maria L. et al. (2011): The role of verbs in indefinite resolution, Handout, 33. DGfS-Jahrestagung, Göttingen.

de Haan, Ger/Weerman, Fred P. (1985): Finiteness and Verb Fronting in Frisian. In: Haider, Hubert/Prinzhorn, Martin (Hg.), Verb Second Phenomena in Germanic Languages, Foris: Dordrecht. 
de Swart, Henriette (1991): Adverbs of quantification: a generalized quantifier approach. Dissertation, Rijkuniversiteit Groningen.

de Swart, Henriette (1998): Introdcution to natural language semantics. Stanford: CSLI Publications.

den Besten, Hans (1983): On the interaction of root transformations and lexical deletive rules. In: Abraham, Werner (Hg.), On the formal syntax of the Westgermania, Amsterdam: John Benjamins, 47-131.

Diesing, Molly (1992): Indefinites. MIT Press.

Dölling, Johannes (2010): Quantitätsimplikaturen. Seminarskript Universität Leipzig, http://www.uni-leipzig.de/ doelling/veranstaltungen/konvimpl2.pdf 23.03.2011.

Ebert, Christian et al. (2006): An information structural account of German integrated verb second clauses. In: Research on Language and Computation 00, 1-24.

Eisenberg, Peter (1989): Grundriß der deutschen Grammatik. Stuttgart: Metzler.

Eisenberg, Peter (2006): Grundriss der deutschen Grammatik. Band 2: Der Satz. Stuttgart: Metzler.

Eisenberg, Peter et al. (2009): Duden. Die Grammatik. Mannheim: Dudenverlag.

Emonds, Joseph E. (1970): Root and structure-preserving transformations. Dissertation, Cambridge, MIT.

Fabricius-Hansen, Cathrine (2013): A text-functional view on syntactic adjunction. Handout, CRC-Tagung Text in Perspective, 29.-30.05.2013, Göttingen.

Farkas, Donka (2002): Specificity distinction. In: Journal of Semantics 19, 213-243.

Fodor, Janet/Sag, Ivan (1982): Referential and quantificational indefinites. In: Linguistics and Philosophy 5, 355-398.

Frank, Nicola (2000): Probleme lexikalischer Selektion und abhängige Verbzweitsätze. In: Linguistische Berichte 184, 469-483.

Frey, Werner (2011): Peripheral adverbial clauses, their licensing and the prefield in Geman. In: Breindl, Eva et al. (Hg.), Satzverknüpfung - Zur Interaktion von Form, Bedeutung und Diskursfunktion, Berlin: de Gruyter, 41-77.

Freywald, Ulrike (2008): Zur Syntax und Funktion von dass-Sätzen mit Verbzweitstellung. In: Deutsche Sprache 36, 246-285.

Freywald, Ulrike (2013): Uneingeleiteter V1- und V2-Satz. In: Meibauer, Jörg et al. (Hg.), Satztypen des Deutschen, Berlin, Boston: de Gruyter, 317-337. 
Gauker, Christopher (1998): What is a context of utterance? In: Philosophical Studies $91,149-172$.

Gaumann, Ulrike (1983): Weil die machen jetzt bald zu. Angabe- und Junktivsatz in der deutschen Gegenwartssprache. Göppingen: Kümmerle.

Geurts, Bart (1999): Presuppositions and Pronouns. Oxford: Elsevier.

Geurts, Bart (2011): Anaphora, accessibility, and bridging. In: Maienborn, Claudia et al. (Hg.), (To appear) Semantics: an international handbook of natural language Meaning, Berlin: de Gruyter.

Geurts, Bart/Maier, Emar (2003): Layered DRT.

Geurts, Bart/van der Sandt, Rob (1999): Domain restriction. In: Bosch, Peter/van der Sandt, Rob (Hg.), Focus. Linguistic, cognitive, and computational perspectives, Cambridge: Cambridge University Press, 268-292.

Günthner, Susanne (1993): „...weil - man kann es ja wissenschaftlich untersuchen“Diskurspragmatische Aspekte der Wortstellung in WEIL-Sätzen. In: Linguistische Berichte 143, 37-59.

Günthner, Susanne (1999a): Wenn-Sätze im Vor-Vorfeld. In: Deutsche Sprache 3, 209-235.

Günthner, Susanne (1999b): Entwickelt sich der Konzessivkonnektor obwohl zum Diskursmarker?: Grammatikalisierungstendenzen im gesprochenen Deutsch. In: Linguistische Berichte 180, 409-446.

Green, Georgia (1976): Main Clause Phenomena in subordinate clauses. In: Language $52,382-397$.

Grewendorf, Günther (1988): Aspekte der deutschen Syntax. Eine RektionsBindungs-Analyse. Tübingen: Narr.

Grewendorf, Günther (2002): Left dislocation as movement. In: Mauck, Simon/Mittelstaedt, Jenny (Hg.), Georgetown University Working Papers in Theoretical Linguistics, vol.2, Fall, Band 2, 31-81.

Grice, Paul (1989): Logic and conversation. In: Grice, Paul (Hg.), Studies in the way of words, Cambridge, Massachusetts: Harvard University Press, 22-40.

Groenendijk, Jeroen A.G./Stokhof, Martin (1985): Studies in the semantics of questions and the pragmatics of answers. Dissertation, University of Amsterdam.

Gärtner, Hans-Martin (2001): Are there V2 relative clauses in German? In: Journal of Comparative Germanic Linguistics 3.2, 97-141. 
Gärtner, Hans-Martin (2002): On the force of V2 declaratives. In: Theoretical Linguistics $28,33-42$.

Gärtner, Hans-Martin/Michaelis, Jens (2010): On modeling the distribution of declarative V2-clauses. The case of disjunction. In: Bab, S./Robering, K. (Hg.), Judgements and propositions. Logical, linguistic, and cognitive issues., Berlin: Logos, $11-25$.

Haegeman, Liliane (2002): Anchoring to speaker, adverbial clauses and the structure of CP. In: Georgetown University Working Papers in Theoretical Linguistics 2, $117-180$.

Haegeman, Liliane (2003): Conditional Clauses: External and Internal Syntax. In: Mind \& Language 18.4, 317-339.

Haegeman, Liliane (2006): Conditionals, factives and the left periphery. In: Lingua 116, 1651-1669.

Haegeman, Liliane (2007): Operator movement and topicalisation in adverbial clauses. In: Folia Linguistica 41, 279-325.

Haegeman, Liliane (2010): The internal syntax of adverbial clauses. In: Lingua 120, 628-648.

Hamblin, Charles L. (1958): Questions. In: Australasian Journal of Philosophy 36, 159-168.

Hamblin, Charles L. (1973): Questions in Montague English. In: Foundations of Language 10, 41-53.

Hara, Yurie (2008): Evidentiality of discourse items and because-clauses. In: Journal of Semantics 25, 229-268.

Hartmann, Dieterich (1984): Reliefgebung: Informationsvordergrund und Informationshintergrund in Texten als Problem von Textlinguistik und Stilistik. In: Wirkendes Wort 34, 305-323.

Heim, Irene (1982): The semantics of definite and indefinite noun phrases. Dissertation, University of Massachusetts, Amherst.

Heim, Irene (1983): File Change Semantics and the Familiarity theory of definiteness. In: Bäuerle, Rainer et al. (Hg.), Meaning, Use and Interpretation of Language, Berlin: de Gruyter, 164-189.

Heim, Irene (1991): Artikel und Definitheit. In: von Stechow, Arnim/Wunderlich, Dieter (Hg.), Semantik. Ein internationales Handbuch der zeitgenössischen Forschung, Berlin: de Gruyter, 487-536. 
Heinämäki, Orvokki (1972): Before. In: Papers from the Eighth Regional Meeting of the Chicago Linguistics Society, University of Chicago, Illinois, 139-151.

Heinämäki, Orvokki (1978): Semantics of English temporal connectives. Indiana: Indiana University Linguistics Club, Bloomington.

Helbig, Gerhard/Buscha, Joachim (1986): Deutsche Grammatik. Ein Handbuch für den Ausländerunterricht. Leipzig: Langenscheidt.

Helbig, Gerhard/Kempter, Fritz (1974): Die uneingeleiteten Nebensätze im Deutsche und ihre Vermittlung im Fremdsprachenunterricht. In: Deutsch als Fremdsprache $11,75-86$.

Heycock, Carolin/Sorace, Antonella (2007): Verb movement in Faroese: New perspectives on an old question. In: Scandinavian Dialect Syntax .

Heycock, Caroline (2005): Embedded Root Phenomena. In: Van Riemsdijk, Henk et al. (Hg.), The Blackwell Companion to Syntax, Oxford: Blackwell, Band II, 174-209.

Höhle, Tilman (1992): Über Verum-Fokus im Deutschen. In: Jacobs, Joachim (Hg.), Informationsstruktur und Grammatik, Opladen: Westdeutscher Verlag, 112-141.

Higginbotham, James (1996): The semantics of questions. In: Lappin, S. (Hg.), The Handbook of contemporary semantic theory, Cambridge, Oxford: Blackwell, 361383 .

Hinrichs, Erhard (1986): Temporal anaphora in discourses of English. In: Linguistics and Philosophy 9, 63-81.

Holler, Anke (2005): Weiterführende Relativsätze. Empirische und theoretische Aspekte. Berlin: Akademie.

Holler, Anke (2008): German dependent clauses from a constraint-based perspective. In: Fabricius-Hansen, Cathrine/Ramm, Wiebke (Hg.), 'Subordination' versus 'Coordination' in Sentence and Text, Amsterdam: John Benjamins, 187-216.

Holler, Anke (2009): Informationsreliefs in komplexen Sätzen: eine diskursrelationale Analyse. In: Ehrich, Veronika et al. (Hg.), Koordination und Subordination im Deutschen. Linguistische Berichte, Sonderheft 16, Hamburg: Buske, 135-159.

Holler, Anke (2013): $d$ - und w-Relativsätze. In: Meibauer, Jörg et al. (Hg.), Satztypen des Deutschen, Berlin, Boston: de Gruyter, 266-300.

Hooper, Joan/A.Thompson, Sandra (1973): On the applicability of root transformations. In: Linguistic Inquiry 4, 465-497.

Horn, Larry R. (1972): On the semantic properties of the logical operators in English. Dissertation, University of California at Los Angeles. 
Horn, Larry R. (1984): Toward a new taxonomy for pragmatic inference: Q-based and R-based implicature. In: Schiffrin, Deborah (Hg.), Meaning, form, and use in context: linguistic applications, Georgetown University Press, 11-42.

Iatridou, Sabine/Kroch, Anthony (1992): The licensing of CP recursion and its relevance to the Germanic verb-second phenomenon. In: Working Papers in Scandinavian Syntax 50, 1-25.

Irmer, Matthias (2009): Bridging reference to eventualities. In: Riester, Arndt/Solstad, Torgrim (Hg.), Proceedings of Sinn und Bedeutung 13 (SuB13), University of Stuttgart, 217-230.

Jacobs, Joachim (1986): Abtönungsmittel als Illokutionstypmodifikatoren. In: Groninger Arbeiten zur Germanistischen Linguistik 27, 100-111.

Johnston, Michael (1994): The syntax and semantics of adverbial adjuncts. Dissertation, UCSC.

Julien, Marit (2007): Embedded V2 in Norwegian and Swedish. In: Working Papers in Scandinavian Syntax. Lund University 80, 103-161.

Kadmon, Nirit (2001): Formal Pragmatics: Semantics, Pragmatics, Presupposition, and Focus. Oxford, Massachusetts: Blackwell.

Kamp, Hans (1981): A theory of truth and semantic representation. In: Groenendijk, Jeroen A.G. et al. (Hg.), Formal Methods in the Study of Language: Proceedings of the Third Amsterdam Colloquium, Part I, Amsterdam: Mathematical Center, 277-321, reprinted in: Groenendijk/Janssen/Stokhof (eds.): Truth, Interpretation and Information, GRASS 2, Foris, 1984, 1-41.

Kamp, Hans/Reyle, Uwe (1993): From Discourse to Logics. Dordrecht: Kluwer.

Kaplan, David (1999): The meaning of ouch and oops (kursiv). Explorations in the theory of meaning as use, los Angeles: University of California.

Karttunen, Lauri (1971): Some observations on factivity. In: Papers in Linguistics $4,55-69$.

Karttunen, Lauri (1973): Presuppositions of compound sentences. In: Linguistic Inquiry $4.2,169-193$.

Karttunen, Lauri (1974): Presuppositions and linguistic context. In: Theoretical Linguistics 1, 181-194.

Karttunen, Lauri (1977): Syntax and Semantics of Questions. In: Linguistics and Philosophy 1, 3-44. 
Keller, Rudi (1993): Das epistemische weil. Bedeutungswandel einer Konjunktion. In: Heringer, Hans Jürgen/Stötzel, Georg (Hg.), Sprachgeschichte und Sprachkritik. Festschrift für Peter von Polenz, de Gruyter, 219-247.

Kiparsky, Paul/Kiparsky, Carol (1970): Fact. In: Bierwisch, Manfred/Heidolph, Karl E. (Hg.), Progress in Linguistics, The Hague: Mouton, 143-173.

Klein, Wolfgang/von Stutterheim, Christiane (1992): Textstruktur und referentielle Bewegung. In: Zeitschrift für Literaturwissenschaft und Linguistik 86, 67-92.

Kliegl, Reinhold et al. (2010): A linear mixed model analysis of masked repetition priming. In: Visual Cognition 18.5, 655-681.

König, Ekkehard (1991): Konzessive Konjunktionen. In: Stechow, Dieter Wunderlich, Arnim von (Hg.), Semantik. Ein internationales Handbuch der zeitgenössischen Forschung, Berlin: de Gruyter, 631-639.

Küper, Christoph (1991): Geht die Nebensatzstellung im Deutschen verloren? Zur pragmatischen Funktion der Wortstellung in Haupt- und Nebensätzen. In: Deutsche Sprache 19, 133-158.

Küper, Christoph (1993): Pragmatische Motiviertheit in der Syntax. Haupt- und Nebensätze im Deutschen. In: Küper, Christoph (Hg.), Von der Sprache zur Literatur. Motiviertheit im sprachlichen und poetischen Kode, Tübingen: Stauffenburg, $37-49$.

Kratzer, Angelika (1991): Modality. In: Stechow, Arnim von/Wunderlich, Dieter (Hg.), Semantik. Ein internationales Handbuch der zeitgenössischen Forschung, Berlin: de Gruyter, 639-650.

Kratzer, Angelika (1999): Beyond ouch and oops (Kursiv). How descriptive and expressive meaning interact. In: Cornell Conference on Theories of Context Dependency, Cornell University, Ithaca, NY.

Krifka, Manfred (2006): Association with focus phrases. In: Molnar, Valerie/Winkler, Susanne (Hg.), The architecture of focus, Berlin, New York: de Gruyter, 105-136.

Krifka, Manfred (2008): Basic notions of information structure. In: Acta Linguistica Hungarica 55, 243-276.

Kruijff-Korbayová, Ivana (2010): Einführung in Pragmatik und Diskurs: Implikaturen. Seminarskript Universität des Saarlands, http://www.coli.unisaarland.de/courses/pd (23.03.2011).

Lakoff, George (1970): Linguistics and Natural Logic. In: Synthese 22, 151-271.

Larson, Richard K. (2004): Sentence-final adverbs and "scope". In: Moulton, Keir/Wolfs, Matthew (Hg.), Proceedings of NELS 34, UMASS: GLSA, 23-43. 
Lascarides, Alex/Oberlander, Jon (1993): Temporal Connectives in a Discourse Context. In: Proceedings of the European Chapter of the Association for Computational Linguistics (EACL93), Utrecht, 260-268.

Löbner, Sebastian (1987): Natural language and generalized quantifier theory. In: Gärdenfors, Peter (Hg.), Generalized quantifiers: Linguistic and logical approaches, Dordrecht: Reidel?, 181-201.

Lehmann, Christian (1984): Der Relativsatz. Typologie seiner Struktur, Theorie seiner Funktionen, Kompendium seiner Grammatik. Narr.

Lewis, David (1975): Adverbs of quantification. In: Keenan, Edward L. (Hg.), Formal semantics of natural language, Cambridge: Cambridge University Press, 3-15.

Lewis, David (1979): Scorekeeping in a language game. In: Journal of Philosophical Logic 8, 339-359.

Lohnstein, Horst (1996): Formale Semantik und natürliche Sprache. Westdeutscher Verlag.

Lohnstein, Horst (2000): Satzmodus - kompositionell. Zur Parametrisierung der Modusphrase im Deutschen. Berlin: Akademie.

Lohnstein, Horst (2004): Variable und invariante Strukturmerkmale von Satzkonnektoren. In: Blühdorn, Hardarik et al. (Hg.), Brücken schlagen. Grundlagen der Konnektorensemantik, Berlin: de Gruyter, 137-160.

Lohnstein, Horst (2013): E- und W-Interrogative. In: Meibauer, Jörg et al. (Hg.), Satztypen des Deutschen, Berlin, Boston: de Gruyter, 51-83.

Lohnstein, Horst/Bredel, Ursula (2004): Inflectional morphology and sentence mood in German. In: Lohnstein, Horst/Trissler, Susanne (Hg.), The syntax and semantics of the left periphery, Berlin: de Gruyter, 235-264.

Maki, Hideki et al. (1999): Embedded topicalization in English and Japanese. In: Lingua 109, 1-14.

Max, Ingolf (2004): Assertion und Präsupposition. Zur Semantik und Pragmatik von Konnektoren. In: Blühdorn, Hardarik et al. (Hg.), Brücken schlagen. Grundlagen der Konnektorensemantik, Berlin: de Gruyter, 93-121.

Mayer, Jörg (1999): Prosodische Merkmale von Diskursrelationen. In: Linguistische Berichte 177, 65-85.

Meibauer, Jörg (1994): Modaler Kontrast und konzeptuelle Verschiebung: Studien zur Syntax und Semantik deutscher Modalpartikeln., Linguistische Arbeiten, Band 314. 
Meinunger, André (2004): Verb position, verbal mood and the anchoring (potential) of sentences. In: Lohnstein, Horst/Trissler, Susanne (Hg.), The syntax and semantics of the left periphery, Berlin, New York: de Gruyter, 313-342.

Meinunger, André (2005): Remarks on the verb second phenomenon, the nature of volitional predicates, 'Konjunktiv' and speculations on illocution, berlin.

Müller, Sonja (2011): (Un)informativität und Grammatik. Extraktion aus Nebensätzen im Deutschen, Studien zur deutschen Grammatik, Band 81. Tübingen: Stauffenburg.

Onea, Edgar (2011): From context to propositions. Handout, Universität Göttingen.

Onea, Edgar (2013): Potential questions in discourse and grammar. Universität Göttingen.

Ormelius-Sandblom, Elisabet (1997): The modal particle schon: Its syntax, semantics and pragmatics. In: Swan, Toril/Westvik, Olaf J. (Hg.), Modality in Germanic Languages. Historical and Comparative Perspectives, de Gruyter, 75-131.

Partee, Barbara (1984): Nominal and temporal anaphora. In: Linguistics and Philosophy $7,243-286$.

Partee, Barbara (1991): Adverbial quantification and event structures. In: Sutton, Laurel et al. (Hg.), Proceedings of the Seventeenth Annual Meeting of the Berkeley Linguistics Society, Berkeley: Berkeley Linguistics Society, 439-456.

Pasch, Renate (1997): Weil mit Hauptsatz - Kuckucksei im denn-Nest. In: Deutsche Sprache 25.3, 252-271.

Pasch, Renate et al. (2003): Handbuch der deutschen Konnektoren. Linguistische Grundlagen der Beschreibung und syntaktische Merkmale der deutschen Satzverknüpfer (Konjunktionen, Satzadverbien und Partikeln). Berlin: de Gryuter.

Pittner, Karin (1999): Adverbiale im Deutschen. Untersuchungen zu ihrer Stellung und Interpretation. Tübingen: Stauffenburg.

Posner, Roland (1972): Theorie des Kommentierens. Frankfurt: Athenäum.

Potts, Christopher (2005): The Logic of Conventional Implicatures. Oxford: Oxford University Press.

Potts, Christopher (2008): Wait a minute! What kind of discourse strategy is this? (Annotated data set), manuscript, UMass Amherst.

Potts, Christopher (2011): Conventional implicature and expressive content. In: Maienborn, Claudia et al. (Hg.), Semantics: an international handbook of natural language meaning, de Gruyter, Band 3. 
Reich, Ingo (2003): Frage, Antwort und Fokus. Berlin: Akademie.

Reich, Ingo/Reis, Marga (2013): Koordination und Subordination. In: Meibauer, Jörg et al. (Hg.), Satztypen des Deutschen, Berlin, Boston: de Gruyter, 536- 569.

Reichenbach, Hans (1947): Elements of Symbolic Logic. London: CollierMcMillan.

Reiners, Ludwig (1951): Der sichere Weg zum guten Deutsch: eine Stilfibel. München: Beck.

Reis, Marga (1992): Zur Grammatik und Pragmatik von Echo-w- Fragen. In: Rosengren, Inger (Hg.), Satz und Illokution 1, Niemeyer, 213-262.

Reis, Marga (1994): Brückeneigenschaften von Matrixsätzen bei langer Extraktion im Deutschen. Handout, 16. DGfS-Jahrestagung, Münster.

Reis, Marga (1995): Extraction from verb-second clauses in German? In: Lutz, Uli/Pafel, Jürgen (Hg.), On extraction and extraposition in German, Amsterdam: John Benjamins, 45-88.

Reis, Marga (1997): Zum syntaktischen Status unselbstständiger Verbzweit-Sätze. In: Dürscheid, Christa et al. (Hg.), Syntax im Fokus, Tübingen: Niemeyer, 112144.

Reis, Marga (2006): Is German V-to-C Movement really semantically motivated? In: Theoretical Linguistics 22, 369-380.

Reis, Marga (2013): „Weil-V2“-Sätze und (k)ein Ende? Anmerkungen zur Analyse von Antomo und Steinbach (2010). In: Zeitschrift für Sprachwissenschaft 32.2, $221-262$.

Reis, Marga/Rosengren, Inger (1991): What do Wh-Imperatives tell us about WhMovement. In: Natural Language and Linguistic Theory 10, 97-118.

Rinas, Karsten (1997): Präsupposition und Komplementierung. Trier: WVT.

Rizzi, Luigi (1997): The fine structure of the left periphery. In: Haegeman, Liliane (Hg.), Elements of Grammar: Handbook in Generative Syntax, Dordrecht: Kluwer, 281-337.

Rizzi, Luigi/Roberts, Ian (1996): Complex Inversion in French. In: Belletti, Luigi, Adriana/Rizzi (Hg.), Parameters and functional heads, Oxford: Oxford University Press, 91-116.

Roberts, Craige (1995): Domain restriction in dynamic semantics. In: Bach, Emmon et al. (Hg.), Quantification in natural language, Dordrecht: Kluwer, 661-700. 
Roberts, Craige (1996): Information Structure: Towards an integrated theory of formal pragmatics. In: Yoon, Jae H./Kathol, Andreas (Hg.), OSU Working Papers in Linguistics 48, 91-136.

Roberts, Craige et al. (2009): Presupposition, Conventional Implicature, and Beyond: A unified account of projection. In: Klinedinst, Nathan/Rothschild, Daniel (Hg.), to appear in: Proceedings of New Directions in the Theory of Presupposition, ESSLLI, Toulouse.

Rooth, Mats (1985): Association with focus. Dissertation, University of Massachusetts, at Amherst,.

Rooth, Mats (1992): A theory of focus interpretation. In: Natural Language Semantics $1,75-116$.

Rothstein, Björn (2007): Tempus. Heidelberg: Winter.

Russell, Bertrand (1905): On Denoting. In: Mind 14.56, 479-493.

Sabel, Joachim (2006): Typologie des W-Fragesatzes. In: Linguistische Berichte 206, 147-195.

Sauerland, Uli (2007): Beyond Unpluggability. In: Theoretical Linguistics 33.2, 231236.

Sawada, Miyuki/Larson, Richard K. (2004): Presupposition \& Root Transforms in Adjunct Clauses. In: Moulton, Keir/Wolfs, Matthew (Hg.), Proceedings of NELS, UMASS: GLSA, Band 34, 517-528.

Schardl, Anisa (2012): Finish-hAn and the QUD, Vortrag auf der 34. DGfSJahrestagung, Frankfurt a.M.

Scheffler, Tatjana (2005): Syntax and semantics of causal denn in German. In: Proceedings of the 15th Amsterdam Colloquium, Amsterdam.

Scheffler, Tatjana (2008): Semantic operators in different dimensions. Dissertation, University of Pennsylvania.

Scheutz, Hannes (1998): Weil-Sätze im gesprochenen Deutsch. In: Hutterer, Claus/Pauritsch, Gertrude (Hg.), Beiträge zur Dialektologie des ostoberdeutschen Raumes, Göppingen: Kümmerle, 85-112.

Schilder, Frank (1999): Presupposition triggered by temporal connectives. In: Proceedings of the TALN`99 workshop Theoretical bases for semantics and pragmatics in NLP, 113-124.

Schilder, Frank (2004): Temporale Konnektoren im Diskurs. In: Blühdorn, Hardarik et al. (Hg.), Brücken schlagen. Grundlagen der Konnektorensemantik, Berlin: de Gruyter, 161-184. 
Schilder, Frank/Tenbrink, Thora (2001): Before and after: sentence-internal and external discourse relations, presented at the workshop from sentence processing to discourse interpretation: Crossing the Borders. Utrecht.

Schlenker, Philippe (2007): Expressive Presuppositions. In: Theoretical Linguistics $33.2,237-245$.

Schlenker, Philippe (2008): Be articulate! A pragmatic theory of presupposition projection. In: Theoretical Linguistics 34, 157-212.

Schwarz, Monika (1998): Referentielle Unterspezifikation in Texten - Zur ErschlieBung nicht explizit eingeführter Referenzobjekte in Peter Bichsels „Ein Tisch ist ein Tisch “. In: Pohl, Inge/Pohl, Jürgen (Hg.), Texte über Texte - Interdisziplinäre Zugänge, Frankfurt a. M.: Peter Lang, 463-472.

Schwarz, Monika (2000): Indirekte Anaphern in Texten. Studien zur domänengebundenen Referenz und Kohärenz im Deutschen. Tübingen: Niemeyer.

Searle, John R. (1969): Speech acts: an essay in the philosophy of language. London: Cambridge University Press.

Seuren, Pieter A. M. (1991): Präsuppositionen. In: von Stechow, Arnim/Wunderlich, Dieter (Hg.), Semantik. Ein internationales Handbuch der zeitgenössischen Forschung, Berlin: de Gruyter, 286-318.

Shannon, Benny (1976): On the two kinds of presupposition in natural language. In: Foundations of Language 14.2, 247-249.

Simons, Mandy (2007): Observations on embedding verbs, evidentiality, and presupposition. In: Lingua 117, 1034-1056.

Simons, Mandy et al. (2011): What projects and why. In: Li, Nan/Lutz, David (Hg.), Proceedings of Semantics and Linguistic Theory (SALT) 20, Ithaca, NY: CLC Publications, 309-327.

Stalnaker, Robert (1974): Pragmatic presuppositions. In: Munitz, Milton K./Unger, Peter (Hg.), Semantics and Philosophy, New York: New York University Press, $47-62$.

Stalnaker, Robert (1978): Assertion. In: Cole, Peter (Hg.), Syntax and semantics: Pragmatics, New York: Academic Press.

Steinbach, Markus (2007): Integrated parentheticals and assertional complements. In: Dehé, Nicole et al. (Hg.), Parentheticals, Amsterdam: Benjamins, 53-87.

Strawson, Peter F. (1950): On Referring. In: Mind. New Series 59.235, 320-344.

Sweetser, Eve E. (1990): From etymology to pragmatics. Metaphorical and cultural aspects of semantic structure. Cambridge: Cambridge University Press. 
Tenbrink, Thora (2004): The German temporal connectors bevor and nachdem in discourse. Handout, 26. DGfS-Jahrestagung, Mainz.

Tenbrink, Thora (2007): Imposing common ground by using temporal connectives: The pragmatics of before and after. In: Fetzer, Anita/Fischer, Kerstin (Hg.), Lexical Markers of Common Ground, Elsevier, 113-139.

Thim-Mabrey, Christiane (1982): Zur Syntax der kausalen Konjunktionen weil, da und denn. In: Sprachwissenschaft 7, 197-219.

Thurmair, Maria (1989): Modalpartikeln und ihre Kombinationen. Tübingen: Niemeyer.

Truckenbrodt, Hubert (2004): Zur Strukturbedeutung von Interrogativsätzen. In: Linguistische Berichte 199, 313-350.

Truckenbrodt, Hubert (2006): On the semantic motivation of syntactic verb movement to $\mathrm{C}$ in German. In: Theoretical Linguistics 22.3, 257-306.

Uhmann, Susanne (1998): Verbstellungsvariation in weil- Sätzen: Lexikalische Differenzierung mit grammatischen Folgen. In: Zeitschrift für Sprachwissenschaft 17.1, 92-139.

Van der Sandt, Rob A. (1992): Presupposition projection as anaphora resolution. In: Journal of Semantics 9, 333-377.

Van der Sandt, Rob A./Geurts, Bart (1991): Presupposition, anaphora, and lexical content. In: Herzog, Otthein/Rollinger, Claus-Rainer (Hg.), Text understanding in LILOG - Integrating Computational Linguistics and Artificial Intelligence Final Report on the IBM Germany LILOG-Project , Berlin: Springer, 259-296.

Van Fraasen, Bas C. (1971): Formal Semantics and Logic. New York: Macmillian.

Vikner, Sten (1995): Verb Movement and Expletive Subjects in the Germanic Languages. New York, Oxford: Oxford University Press.

Volodina, Anna (2008): Zur syntaktischen und prosodischen Markierung pragmatischer Phänomene im Bereich der Konnektorenforschung. In: Péteri, Attila (Hg.), An der Grenze zwischen Grammatik und Pragmatik, Frankfurt a. M.: Lang.

Volodina, Anna (2011): Konditionalität und Kausalität im Diskurs. Eine korpuslinguistische Studie zum Einfluss von Syntax und Prosodie auf die Interpretation komplexer Äußerungen. Nummer 54 in Studien zur deutschen Sprache, Tübingen: Narr.

Volodina, Anna (2013): Konditionale V2-Nebensätze, vortrag auf der 5. Tagung Semantik und Pragmatik im Südwesten, Saarbrücken. 
von Fintel, Kai (2000): What is presupposition accommodation?, unveröffentlichtes Manuskript, MIT.

von Fintel, Kai (2004a): A minimal theory of adverbial quantification. In: Partee, Barbara/Kamp, Hans (Hg.), Context dependence in the analysis of linguistic meaning, Amsterdam: Elsevier, 137-175.

von Fintel, Kai (2004b): Would you believe it? The king of France is back! (Presuppositions and truth-value intuitions). In: Bezuidenhout, Anne/Reimer, Marga (Hg.), Descriptions and beyond: an interdisciplinary collection of essays on definite and indefinite descriptions and other related phenomena, Oxford: Oxford University Press, 315-341.

von Heusinger, Klaus (2002): Specificity and Definiteness in Sentence and Discourse Structure. In: Journal of Semantics 19, 245-274.

von Heusinger, Klaus (2011): Specificity. In: von Heusinger, Klaus et al. (Hg.), Semantics: An International Handbook of Natural Language Meaning, Berlin: de Gruyter, Band 2, 1024-1057.

von Stechow, Arnim (1990): Focusing and Backgrounding Operators. In: Abraham, Werner (Hg.), Discourse particles - Descriptive and theoretical investigations on the logical, syntactic and pragmatic properties of discourse particles in German, Amsterdam: John Benjamins, 37-84.

von Stutterheim, Christiane (1989): Quaestio und referentielle Bewegung in Erzählungen. In: Linguistische Berichte 109, 163-183.

von Stutterheim, Christiane/Klein, Wolfgang (1989): Referential Movement in Descriptive and narrative discourse. In: Dietrich, Rainer/Graumann, Carl F. (Hg.), Language processing in social context, Elsevier, 39-76.

Wechsler, Stephen (1991): Verb second and illocutionary force. In: Leffel, Katherine/Bouchard, Denis (Hg.), Views on Phrase Structure, Dordrecht: Kluwer, 177 191.

Weerman, Fred (1989): The V2 conspiracy. A synchronic and diachronic analysis of verbal positions in Germanic languages. Dordrecht: Foris.

Wegener, Heide (1993): Weil- das hat schon seinen Grund. Zur Verbstellung in Kausalsätzen mit weil im gegenwärtigen Deutsch. In: Deutsche Sprache 21, 289-305.

Wegener, Heide (2000): Da, denn und weil - der Kampf der Konjunktionen. Zur Grammatikalisierung im kausalen Bereich. In: Thieroff, Rolf (Hg.), Deutsche Grammatik in Theorie und Praxis, Tübingen: Niemeyer, 69-81.

Weinrich, Harald (1964): Tempus. Besprochene und erzählte Welt. Stuttgart: Kohlhammer. 
Wiklund, Anna-Lena et al. (2009): On the distribution and illocution of V2 in Scandinavian that-clauses. In: Lingua 12, 1914-1938.

Wöllstein, Angelika/Reis, Marga (2010): Zur Grammatik (vor allem) konditionaler V1-Gefüge im Deutschen. In: Zeitschrift für Sprachwissenschaft 29.1, 111-179.

Xue, Jingyang/Onea, Edgar (2011): Correlation between presupposition projection and at-issueness: an empirical study. In: Erscheint in: Proceedings of ESSLLI 2011.

Zifonun, Gisela et al. (Hg.) (1997): Grammatik der deutschen Sprache. Berlin, New York: de Gruyter.

Zimmermann, Malte (2004): Zum Wohl: Diskurspartikeln als Satztypmodifikatoren. In: Linguistische Berichte 199, 253-286. 